Exploring the genetics underlying the responses to consecutive combinations of biotic stresses and drought in Arabidopsis thaliana

Pingping Huang 
Thesis committee

\section{Promotor}

Prof. Dr M. Koornneef

Personal chair at the Laboratory of Genetics

Wageningen University

\section{Co-promotor}

Dr M.G.M. Aarts

Associate Professor, Laboratory of Genetics

Wageningen University

\section{Other members}

Prof. Dr FPM Govers, Wageningen University

Prof. Dr R Pierik, Utrecht University

Prof. Dr H.J. Bouwmeester, Wageningen University

Dr CG van der Linden, Wageningen University

This research was conducted under the auspices of the Graduate School of Experimental Plant Sciences 


\title{
Exploring the genetics underlying the responses to consecutive combinations of biotic stresses and drought in Arabidopsis thaliana
}

\author{
Pingping Huang
}

\section{Thesis}

submitted in fulfilment of the requirements for the degree of doctor

at Wageningen University

by the authority of the Rector Magnificus

Prof. Dr A.P.J. Mol,

in the presence of the

Thesis Committee appointed by the Academic Board

to be defended in public

on Friday 7 October 2016

at 11 a.m. in the Aula. 
Pingping Huang

Exploring the genetics underlying the responses to consecutive combinations of biotic stresses and drought in Arabidopsis thaliana

291 pages.

PhD thesis, Wageningen University, Wageningen, NL (2016)

With references, with summary in English and Chinese

ISBN: $978-94-6257-859-3$

DOI: $10.18174 / 385576$ 
per La meglio gioventù 


\section{Content}

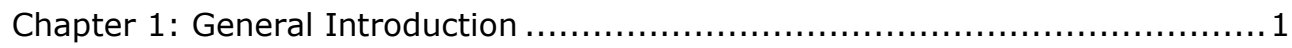

Chapter 2: Genetic architecture of plant stress resistance: multi-trait genomewide association mapping ........................................................ 17

Chapter 3: Genome wide association analysis reveals candidate genes controlling a negative effect of biotic stresses on consecutive osmotic stress response

Chapter 4: Genome wide association mapping of the Arabidopsis thaliana growth response to the sequential combination of drought and Botrytis cinerea infection 103

Chapter 5: Transcriptome dynamics of Arabidopsis during sequential biotic and

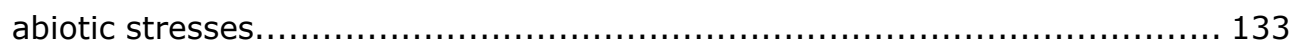

Chapter 6: Opposite interaction effects on the ethylene signalling pathway of two biotic stresses (Botrytis cinerea and Pieris rapae) on subsequent drought stress responses in Arabidopsis thaliana 181

Chapter 7: Phenotypic and genetic responses of Arabidopsis thaliana natural accessions to consecutive Pieris rapae herbivory and drought stress .......... 219

Chapter 8: General discussion ................................................... 255

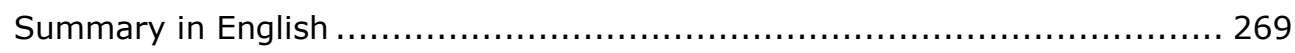

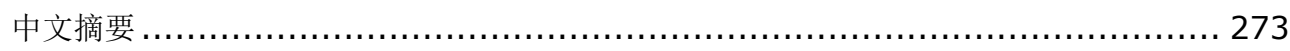

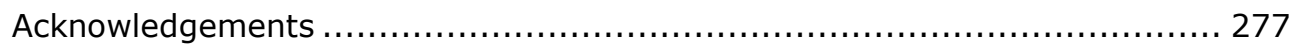

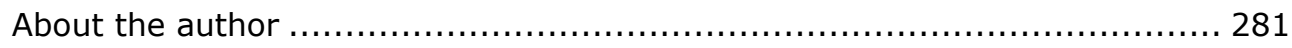

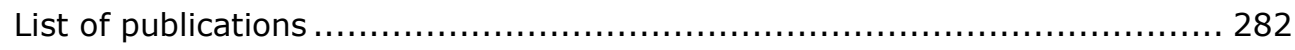

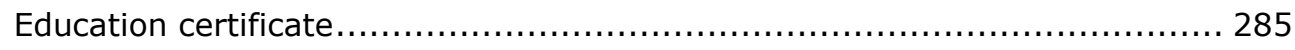

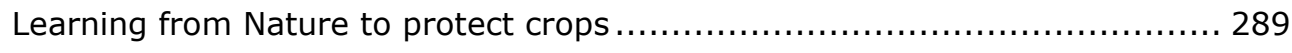




\section{List of Abbreviations:}

FW Rosette fresh weight

DW Rosette dry weight

WC Water content in rosette

PLA Projected rosette area

ACC Accessions

GWA Genome-wide association

QTL Quantitative trait loci

GxE Genotype by environment interaction 


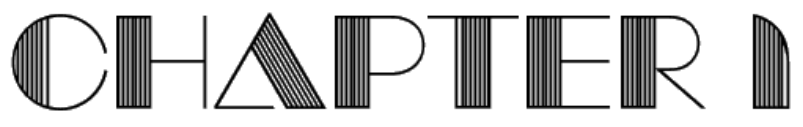

\section{General Introduction}

Pingping Huang 
Plants growing in natural environments are exposed to a broad range of biotic and abiotic factors that are known to cause stress symptoms in many species. These stresses can be: pathogen attack, insect herbivory, drought, extreme temperatures, UV radiation, salinity, etc. (Pareek et al., 2010; RobertSeilaniantz et al., 2010). Biotic and abiotic stress-inducing determinants often adversely impact plant growth and development, frequently leading to severe annual yield losses in agricultural production (Pierik et al., 2013; Pieterse et al., 2012; Stam et al., 2014).

\section{Stress responses in plants}

In response to a myriad of biotic and abiotic stress triggers, plants mobilize signaling molecules, thereby boosting the accumulation of so-called second messengers such as reactive oxygen species (ROS), calcium ions $\left(\mathrm{Ca}^{2+}\right)$, phospholipids, mitogen-activated protein kinases (MAPKs), G-proteins, and phytohormones (Smékalová et al., 2014; Takahashi et al., 2011; Tuteja and Sarvajeet, 2012). Various stress signaling pathways are interconnected in an overarching network, influenced by key regulators, such as transcription factors and MAPKs, responding to, and affecting the production of stress-related plant hormones and ROS (Caarls et al., 2015; Fujita et al., 2006; Pieterse et al., 2009b; Rejeb et al., 2014; Robert-Seilaniantz et al., 2011). Under stress conditions, ROS are generated rapidly and act as a common message to activate MAPKs (Jalmi and Sinha, 2015). MAPK- and $\mathrm{Ca}^{2+}$-induced activation of the calcium-dependent protein kinases (CDPKs) are two major signaling cascades triggered in response to both biotic and abiotic stresses (Colcombet and Hirt, 2008). The last step of the delineated defense mechanism is the subsequent induction of transcription of stress-responsive genes. While the resultant production of an array of plant hormones has been associated with a variety of stress triggers (of both biotic and abiotic nature), high accumulation of abscisic acid (ABA) was recorded under specific abiotic conditions, such as drought, osmotic imbalance, salinity, and low temperature (Tuteja, 2007). Further, this hormone was shown to negatively interact with biotic stress response cascades. For instance, the drought-associated accumulation of ABA coincided with decreased levels of salicylic acid (SA), jasmonic acid (JA), and ethylene (ET) (Adie et al., 2007; Koornneef and Pieterse, 2008). Moreover, ABA can modulate 
the crosstalk between the JA-regulated signaling pathway and those under control of salicylic acid (SA), gibberellins (GAs), and auxins (AUXs) (Abe et al., 2003; Dombrecht et al., 2007; Kazan and Manners, 2013; Yamaguchi-Shinozaki and Shinozaki, 2005).

\section{Genes involved in response to biotic and abiotic stress}

Reactive oxygen species (ROS) as stress-response gene activators

In the model plant Arabidopsis thaliana (hereafter Arabidopsis), a few hundred genes involved in the ROS scavenging network were identified (Mittler et al., 2004). Among them, SUPEROXIDE DISMUTASE 2 (FSD2), FSD3, ASCORBATE PEROXIDASE 1 (APX1), APX2, and MONODEHYDROASCORBATE REDUCTASE 4 (MDAR4) were shown to be transcriptionally induced upon heat, cold, drought, and salinity stresses (Mittler et al., 2004). Furthermore, zinc finger and WRKY transcription factor genes (ZF- and WRKY-TFs) are induced by ROS. For example, the zinc finger protein ZAT12 is required for the expression of $A P X 1$ and responds transcriptionally to multiple triggers, including intense light, freezing, osmotic and oxidative stress, wounding, and pathogen infection (Fujita et al., 2009; Rizhsky et al., 2004), while another zinc finger protein, ZAT6, positively regulates responses to drought, salinity, as well as bacterial infection (Shi et al., 2014). Concurrently, WRKY25 is transcriptionally induced under multiple conditions, such as wounding, heat, osmotic and oxidative stress, and appears to be regulated by ZAT12 (Rizhsky et al., 2004), whereas WRKY70 interacts with ZAT7 in regulating plant disease response and the SA- and JAmediated signaling pathways (Li et al., 2004) .

\section{Mitogen-activated protein kinases (MAPKs)}

MAPKs often engage in cross-talk with ROS signaling pathways. For instance, the OXIDATIVE SIGNAL-INDUCIBLE 1 (OXI1) kinase plays a role in ROS sensing and is required for activation of MPK3 and MPK6 (Forzani et al., 2011; Smékalová et al., 2014). These two are involved in disease resistance, drought and salt tolerance, and abscisic acid (ABA) signaling (Frei dit Frey et al., 2014; Tsugama et al., 2012; Yu et al., 2010). Moreover, MITOGEN-ACTIVATED PROTEIN KINASE KINASE KINASE 1 (MEKK1), a common MAP kinase kinase kinase (MPKKK), is activated by ROS under various biotic and abiotic conditions. 
In biotic stress situations, MEKK1 mobilizes MKK4/5 and stimulates expression of $M P K 3 / 6$ for innate immunity response, while under abiotic stress, the kinase activates MKK1/MKK2 for the expression of MPK4 and MPK6, thereby affording cold and salt acclimation (Jalmi and Sinha, 2015).

\section{Hormone signaling-regulated transcription factors}

Transcription factors, such as ERF1, BOS1, RD26, MYB2, MYC2, RD22, ANAC019 and ANAC055 are associated with both ABA and JA-ET signaling (Koornneef and Pieterse, 2008; Shinozaki and Yamaguchi-Shinozaki, 2007). They are expressed under multiple biotic and abiotic stresses, including drought, salinity, osmotic imbalance, as well as infection by Fusarium oxysporum and Pieris rapae herbivory (Abe et al., 2003; Anderson et al., 2004; Fujita et al., 2004; Shinozaki and Yamaguchi-Shinozaki, 2007; Vos et al., 2013). Further, multiple transcription factors regulate the expression of defense- and woundingresponsive genes. For instance, ANAC019 and ANAC055 are expressed under drought stress, they interact with MYB2 and MYC2 to up-regulate the expression of wounding-responsive genes VSP1 and LOX2 (Bu et al., 2008; Kazan and Manners, 2013).

\section{Cross-talk between drought, pathogen, and insect herbivore response mechanisms}

Drought is one of the most ubiquitous negative abiotic environmental factors influencing plants. Under drought stress, the accumulation of ABA triggers stomatal closure to reduce water loss via transpiration (Rai and Takabe, 2006; Xiong and Ishitani, 2006). As a consequence, water uptake and assimilation are diminished, resulting in lower nutrient take up, which leads to lower biomass (Verslues et al., 2006). Adaptive responses to drought involve metabolic, osmotic, and structural adjustment, as well as production of proteins with DNA damage-control and DNA repair functions (Ingram and Bartels, 1996). ABAdependent and ABA-independent drought-response signaling pathways were described by Shinozaki and Yamaguchi-Shinozaki (2007). In addition, JA and ET have been implicated as important regulators of drought response (Bray, 1997; Shinozaki et al., 2003; Riera et al., 2005; Huang et al., 2008). 
Plant pathogenic fungi can generally be classified as necrotrophs or biotrophs depending on whether they kill their host cells or not. Botrytis cinerea (hereafter referred to as Botrytis) is a necrotrophic fungus and is considered to be the second most important plant pathogen (Dean et al., 2012), infecting over 200 cultivated plant species and causing significant economic damage to crops worldwide. It kills plant tissue prior to feeding deploying diverse mechanisms that cause decay, e.g., enzymatic degradation of the cell wall, generation of toxic reactive oxygen compounds, and secretion of host non-selective toxins (Diaz et al., 2002; El Oirdi et al., 2011; Geraats et al., 2002; Rowe et al., 2010; Thomma et al., 1998; Thomma et al., 1999). While plant defense against biotrophic pathogens commonly involves SA, fighting necrotrophic invaders often requires the action of JA and ET (Pieterse et al., 2009). ABA and SA have been shown to exert negative effects on resistance to necrotrophic fungi (El Oirdi et al., 2011; Liu et al., 2015; Vos et al., 2015).

Insect herbivores consume over $15 \%$ of the plant biomass produced annually in temperate and tropical ecosystems, making insect herbivory a major conduit by which energy flows through food chains (Agrawal, 2011; Cyr and Pace, 1993; Johnson, 2011). JA is an important primary signal in herbivoreinduced local and systemic defense mechanisms in various plant-herbivore interactions, while $A B A$ plays a modulating role in the JA-responsiveness (Bodenhausen and Reymond, 2007; Howe and Jander, 2008; Soler et al., 2013; Vos et al., 2013). Pieris rapae (hereafter Pieris), the Small Cabbage White butterfly, is among the most destructive pests of cruciferous plants as it has adapted to the glucosinolate-derived glycoside toxins produced by crucifers as chemical deterrents (Hopkins et al., 2009). SA is reported to inhibit the JAdependent defense pathway induced by Pieris feeding (Koornneef et al., 2008).

One of the signaling pathways that modulate the cross-talk between Botrytis, Pieris, and drought stresses is the MYC2-related signaling pathway. MYC2 is a positive regulator of drought stress response induced by $A B A$ as well as a negative regulator of the Botrytis defense gene PDF1.2 through interaction with ERF1 within the JA-signaling pathway. What is more, MYC2 down-regulates the expression of other pathogen defense genes, such as $P R 3$ and $P R 4$, while 
boosting that of the Pieris defense-responsive gene VSP2 (Anderson et al., 2004; Lorenzo et al., 2004;Dombrecht et al., 2007; Vos et al., 2013).

\section{Effects of consecutive stresses on plants}

Abiotic stresses can significantly affect plant responses to biotic strains and vice versa, depending on the timing, nature, and severity of the afflicting events (Atkinson and Urwin, 2012; Appel et al., 2014; Rejeb et al., 2014). Both positive and negative interactions among various stress factor combinations have been reported (Suzuki et al., 2014). For instance, a drought-stress-associated increase in cellular ABA concentrations causes plant stomata to close, which has a negative impact on photosynthesis. This often leads to elevated canopy temperatures and reduced relative humidity of the afflicted plant, which may render its microenvironment suboptimal for herbivores, adversely influencing their fitness and reproduction (Haile, 2000). At the gene level, drought-induced accumulation of ABA leads to up-regulation of specific transcription factors, such as CBF, NAC, and MYB. The TF activation, in turn, triggers elevated expression of pathogenesis-related (PR) genes, thus rendering the plant resistant to subsequent biotic stress. For example, the drought tolerance gene Di19-encoded ZF-TF modulates the expression of PR1, PR2, and PR5 in A. thaliana (Liu et al., 2013).

Furthermore, increased ABA concentrations have a positive impact on callose deposition, which may boost plant resistance to fungal and bacterial pathogens (Mauch-Mani and Mauch, 2005). Studies with tomato plants showed that a drought-associated surge in endogenous ABA levels caused a $50 \%$ reduction in Botrytis infection (Achuo, 2006), while moderate drought stress in Nicotiana benthamiana led to decreased growth of its bacterial invader, Pseudomonas syringae pv. tabaci (Ramegowda et al., 2013).

On the other hand, exposure to herbivorous insects and pathogens can have both positive and negative impacts on plant response to subsequent drought stress (Ramegowda and Senthil-Kumar, 2015). For example, Brome Mosaic Virus (BMV), Cucumber Mosaic Virus (CMV), and Tobacco Mosaic Virus (TMV) infections enhance drought tolerance of $N$. benthamiana through reduction of 
transpiration rate and a boost in osmoprotectant levels (Xu et al., 2008). The reverse is true of the infection of $A$. thaliana by the Turnip Mosaic Virus (TuMV), which causes lesions, mosaics, and mottling that reduce photosynthetic capacity, thereby rendering the plant more susceptible to subsequent drought stress (Prasch and Sonnewald, 2015).

\section{Tailored responses of plants to stress combinations}

Generally, plant responses to individual stress factors are different from those involved in countering a simultaneous or sequentially combination of detrimental triggers. Most studies have focused on a single time point in the stress response, representing only a snapshot of the transcriptional changes that are induced by a single or combinatorial stress (Stein and Waters, 2011; Shaik and Ramakrishna, 2013). Coding sequences involved in response to multiple factors can be identified through comparison of differentially expressed genes (DEGs) under various individual and combined stress treatments (Prasch and Sonnewald, 2013; Rasmussen et al., 2013). Transcriptome analysis on simultaneous effects of drought, heat, and virus infections in Arabidopsis resulted in the identification of 11 genes involved in all relevant single, double, and triple stress treatments (Prasch and Sonnewald, 2013). It was impossible though to determine how single stress responses led to responses to stress factor combinations. For instance, expression of the resistance gene RPS6 was only responsive in case of simultaneous heat, drought, and virus treatments, but not under any of the double or individual stresses (Rasmussen et al., 2013a). As the responses of plants to blends of triggering factors cannot be predicted based on mechanisms countering their individual components, there is likely to be a unique combinatorial stress response, as has been suggested by Ramegowda and Senthil-Kumar (2015). 


\section{RNA-sequencing and genome-wide association}

\section{mapping}

Microarray and RNA-sequencing (RNA-seq) approaches are very useful in providing large, yet comprehensive, sets of gene expression data. However, for financial reasons, this strategy can be applied to examine but a limited number of genotypes at once. Natural variation, partly reflecting the adaptation to different environments, is one of the most important resources for genetic studies in Arabidopsis (Koornneef et al., 2004; Alonso-Blanco et al., 2005; Weigel, 2012). Due to the aforementioned constraints, most investigative efforts addressing plant responses to multiple individual stresses have focused on only a few genotypes (Stein and Waters, 2011; Rasmussen et al., 2013; Shaik and Ramakrishna, 2013). To fully understand the genetic architecture of complex traits, such as plant adaptation to a diversity of stress factors, a much wider survey of the natural genetic variation within a species is mandatory (if variation is present). Genome-wide association (GWA) analysis, relying on a large number of well-genotyped plant accessions, provides an important tool for natural genetic variation research approaches. Although the interest in natural variation and GWA mapping is rapidly increasing (Wijnen and Keurentjes, 2014; Ogura and Busch, 2015), no comprehensive evaluation of natural genetic variation in plants tolerant to simultaneous and sequential biotic and abiotic stress factor combinations was available at the time the hereby presented $\mathrm{PhD}$ research project had begun. 


\section{Scope of the thesis}

In the research endeavors described in this thesis, Arabidopsis was used as a model organism to study plant responses to different sequential combinations of biotic factors (infection with Botrytis or herbivory by Pieris) and drought. The main objective was to identify genes that contribute to tolerance to the aforementioned sequential stress combinations.

The investigations described herein are part of a collaborative project, involving three PhD students, embedded within the national 'Learning from Nature' research program. My contribution involved application of large-scale experimental approaches to examine the genetics and physiology of variation in plant tolerance to sequential combinations of the indicated biotic/abiotic factors, mainly focusing on the response to drought or osmotic stress, in combination with biotic factors. With three single stress-inducing determinants, i.e., drought or osmotic stress, Pieris herbivory and Botrytis infection, six sequential combinations of treatments could be applied. RNA-seq was used to analyze the dynamics of transcriptome changes over time that occurred in Arabidopsis accession Col-0 in response to the single and combined treatments, whereas the GWA approach was employed to identify genes characterized by natural genetic variation contributing to different phenotypic responses to the investigated trigger combinations.

The main results of the 'Learning from Nature' program, integrating the GWA analyses of the aforementioned large set of Arabidopsis accessions (the HapMap population) challenged with 11 single environmental factors and several of their consecutive combinations, are reported in a comprehensive, multiauthor paper presented as Chapter 2.

Responses to the consecutive biotic and drought/osmotic stresses were mapped using two types of the GWA methods. Genotype-by-environment ( $\mathrm{G} \times \mathrm{E}$ ) effects and phenotypic plasticity of several singe nucleotide polymorphisms (SNPs) relevant to stress responses were highlighted and the biological functions of some candidate genes were validated by gene expression and mutant analyses in Chapter $\mathbf{3 .}$ 
Responses to consecutive drought and Botrytis infection were delineated using univariate GWA-mapping. Investigation results pertaining to phenotypic plasticity of significant SNPs as well as biological function of selected candidate genes are presented in Chapter 4.

Major outcomes of the whole-transcriptome profile analysis of Arabidopsis Col-0 exposed to three single stress triggers (Pieris, Botrytis, drought) and six consecutive combinations thereof are reported in a multi-author paper presented as Chapter 5.

The most relevant or significant findings of the whole-transcriptome profiling of Arabidopsis Col-0 exposed to biotic and subsequent drought stresses and the alternative splicing (AS) events identified under different conditions were investigated in detail and are described in Chapter 6.

Phenotypic responses of 16 Arabidopsis accessions challenged with different single or combinatorial stresses and the changes in expression levels of nine drought-responsive genes under alternative stress conditions are expounded upon in Chapter 7.

All findings presented in this thesis, in concert with their implications for future research, are comprehensively discussed in Chapter $\mathbf{8}$. 


\section{References}

Abe, H., Urao, T., Ito, T., Seki, M., Shinozaki, K. and Yamaguchi-Shinozaki, K. (2003). Arabidopsis AtMYC2 (bHLH) and AtMYB2 (MYB) function as transcriptional activators in abscisic acid signaling. Plant Cell 15, 63-78.

Adie, B. A., Perez-Perez, J., Perez-Perez, M. M., Godoy, M., SanchezSerrano, J. J., Schmelz, E. A. and Solano, R. (2007). ABA is an essential signal for plant resistance to pathogens affecting JA biosynthesis and the activation of defenses in Arabidopsis. Plant Cell 19, 1665-81.

Agrawal, A. A. (2011). Current trends in the evolutionary ecology of plant defence. Functional Ecology 25, 420-432.

Alonso-Blanco, C., Gomez-Mena, C., Llorente, F., Koornneef, M., Salinas, J. and Martinez-Zapater, J. M. (2005). Genetic and molecular analyses of natural variation indicate CBF2 as a candidate gene for underlying a freezing tolerance quantitative trait locus in Arabidopsis. Plant Physiol 139.

Anderson, J. P., Badruzsaufari, E., Schenk, P. M., Manners, J. M., Desmond, O. J., Ehlert, C., Maclean, D. J., Ebert, P. R. and Kazan, K. (2004). Antagonistic interaction between abscisic acid and jasmonate-ethylene signaling pathways modulates defense gene expression and disease resistance in Arabidopsis. Plant Cell 16, 3460-79.

Appel, H. M., Maqbool, S. B., Raina, S., Jagadeeswaran, G., Acharya, B. R., Hanley, J. C., Miller, K. P., Hearnes, L., Jones, A. D., Raina, R. et al. (2014). Transcriptional and metabolic signatures of Arabidopsis responses to chewing damage by an insect herbivore and bacterial infection and the consequences of their interaction. Frontiers in Plant Science 5, 441.

Atkinson, N. J. and Urwin, P. E. (2012). The interaction of plant biotic and abiotic stresses: from genes to the field. Journal of Experimental Botany 63, 3523-3543.

Bodenhausen, N. and Reymond, P. (2007). Signaling pathways controlling induced resistance to insect herbivores in Arabidopsis. Molecular Plant-Microbe Interactions 20, 1406-1420.

Bray, E. A. (1997). Plant responses to water deficit. Trends in Plant Science 2, 48-54.

Bu, Q., Jiang, H., Li, C. B., Zhai, Q., Zhang, J., Wu, X., Sun, J., Xie, Q. and Li, C. (2008). Role of the Arabidopsis thaliana NAC transcription factors ANAC019 and ANAC055 in regulating jasmonic acid-signaled defense responses. Cell Res 18, 756-67.

Caarls, L., Pieterse, C. M. J. and Van Wees, S. C. M. (2015). How salicylic acid takes transcriptional control over jasmonic acid signaling. Frontiers in Plant Science 6.

Colcombet, J. and Hirt, H. (2008). Arabidopsis MAPKs: a complex signalling network involved in multiple biological processes. Biochem Journal 413, 217-26.

Cyr, H. and Pace, M. L. (1993). Magnitude and patterns of herbivory in aquatic and terrestrial ecosystems. Nature 361, 148-150.

Dean, R., Van Kan, J. A. L., Pretorius, Z. A., Hammond-Kosack, K. E., Di Pietro, A., Spanu, P. D., Rudd, J. J., Dickman, M., Kahmann, R., Ellis, J. et al. (2012). The Top 10 fungal pathogens in molecular plant pathology. Molecular Plant Pathology 13, 414-430.

Diaz, J., Ten Have, A. and Van Kan, J. A. L. (2002). The role of ethylene and wound signaling in resistance of tomato to Botrytis cinerea. Plant Physiology 129, 13411351.

Dombrecht, B., Xue, G. P., Sprague, S. J., Kirkegaard, J. A., Ross, J. J., Reid, J. B., Fitt, G. P., Sewelam, N., Schenk, P. M., Manners, J. M. et al. (2007). MYC2 differentially modulates diverse jasmonate-dependent functions in Arabidopsis. Plant Cell 19, 2225-45.

El Oirdi, M., Abd El Rahman, T., Rigano, L., El Hadrami, A., Rodriguez, M. C., Daayf, F., Vojnov, A. and Bouarab, K. (2011). Botrytis cinerea manipulates the antagonistic effects between immune pathways to promote disease development in tomato. Plant Cell 23, 2405-2421. 
Forzani, C., Carreri, A., de la Fuente van Bentem, S., Lecourieux, D., Lecourieux, F. and Hirt, H. (2011). The Arabidopsis protein kinase Pto-interacting 1-4 is a common target of the oxidative signal-inducible 1 and mitogen-activated protein kinases. FEBS Journal 278, 1126-1136.

Frei dit Frey, N., Garcia, A. V., Bigeard, J., Zaag, R., Bueso, E., Garmier, M., Pateyron, S., de Tauzia-Moreau, M. L., Brunaud, V., Balzergue, S. et al. (2014). Functional analysis of Arabidopsis immune-related MAPKs uncovers a role for MPK3 as negative regulator of inducible defences. Genome Biology 15, R87.

Fujita, M., Fujita, Y., Noutoshi, Y., Takahashi, F., Narusaka, Y., Yamaguchi-Shinozaki, K. and Shinozaki, K. (2006). Crosstalk between abiotic and biotic stress responses: a current view from the points of convergence in the stress signaling networks. Current Opinion in Plant Biology 9, 436-442.

Fujita, Y., Fujita, M., Yamaguchi-Shinozaki, K. and Shinozaki, K. (2009). Transcription Factors Involved in the Crosstalk between Abiotic and Biotic Stress-Signaling Networks. In Signal Crosstalk in Plant Stress Responses, pp. 43-58: Wiley-Blackwell.

Geraats, B. P. J., Bakker, P. A. H. M. and Van Loon, L. C. (2002). Ethylene insensitivity impairs resistance to soilborne pathogens in tobacco and Arabidopsis thaliana. Molecular Plant-Microbe Interactions 15, 1078-1085.

Hopkins, R. J., Van Dam, N. M. and Van Loon, J. J. A. (2009). Role of glucosinolates in insect-plant relationships and multitrophic interactions. Annual Review of Entomology 54, 57-83.

Howe, G. A. and Jander, G. (2008). Plant immunity to insect herbivores. Annual Review of Plant Biology 59, 41-66.

Huang, D. Q., Wu, W. R., Abrams, S. R. and Cutler, A. J. (2008). The relationship of drought-related gene expression in Arabidopsis thaliana to hormonal and environmental factors. Journal of Experimental Botany 59, 2991-3007.

Ingram, J. and Bartels, D. (1996). The molecular basis of dehydration tolerance in plants. Annual Review of Plant Physiology and Plant Molecular Biology 47, 377-403.

Jalmi, S. K. and Sinha, A. K. (2015). ROS mediated MAPK signaling in abiotic and biotic stress- striking similarities and differences. Front Plant Science 6, 769.

Johnson, M. T. J. (2011). Evolutionary ecology of plant defences against herbivores. Functional Ecology 25, 305-311.

Kazan, K. and Manners, J. M. (2013). MYC2: the master in action. Molecular Plant 6, 686-703.

Koornneef, A., Leon-Reyes, A., Ritsema, T., Verhage, A., Den Otter, F. C., Van Loon, L. C. and Pieterse, C. M. J. (2008). Kinetics of salicylate-mediated suppression of jasmonate signaling reveal a role for redox modulation. Plant Physiology 147, 1358-1368.

Koornneef, A. and Pieterse, C. M. (2008). Cross talk in defense signaling. Plant Physiology 146, 839-44.

Koornneef, M., Alonso-Blanco, C. and Vreugdenhil, D. (2004). Naturally occurring genetic variation in Arabidopsis thaliana. Annual Review of Plant Biology 55, 141-72.

Li, J., Brader, G. and Palva, E. T. (2004). The WRKY70 Transcription Factor: A Node of Convergence for Jasmonate-Mediated and Salicylate-Mediated Signals in Plant Defense. Plant Cell 16, 319-331.

Liu, S., Kracher, B., Ziegler, J., Birkenbihl, R. P. and Somssich, I. E. (2015). Negative regulation of ABA signaling by WRKY33 is critical for Arabidopsis immunity towards Botrytis cinerea 2100. eLife 4, e07295.

Liu, W. X., Zhang, F. C., Zhang, W. Z., Song, L. F., Wu, W. H. and Chen, Y. F. (2013). Arabidopsis Di19 functions as a transcription factor and modulates PR1, PR2, and PR5 expression in response to drought stress. Molecular Plant 6, 1487-502.

Lorenzo, O., Chico, J. M., Sanchez-Serrano, J. J. and Solano, R. (2004). JASMONATE-INSENSITIVE1 encodes a MYC transcription factor essential to discriminate between different jasmonate-regulated defense responses in Arabidopsis. Plant Cell 16, 1938-50. 
Mauch-Mani, B. and Mauch, F. (2005). The role of abscisic acid in plantpathogen interactions. Current Opinion in Plant Biology 8, 409-414.

Mittler, R., Vanderauwera, S., Gollery, M. and Van Breusegem, F. (2004). Reactive oxygen gene network of plants. Trends in Plant Science 9, 490-498.

Ogura, T. and Busch, W. (2015). From phenotypes to causal sequences: using genome wide association studies to dissect the sequence basis for variation of plant development. Current Opinion in Plant Biology 23, 98-108.

Pareek, A., Sopory, S. K., Bohnert, H. J. and Govindjee. (2010). Abiotic Stress Adaptation in Plants: Physiological, Molecular and Genomic Foundation. Dordrecht: Springer Science+Business Media B.V.

Pierik, R., Mommer, L. and Voesenek, L. A. C. J. (2013). Molecular mechanisms of plant competition: neighbour detection and response strategies. Functional Ecology 27, 841-853.

Pieterse, C. M., Van der Does, D., Zamioudis, C., Leon-Reyes, A. and Van Wees, S. C. (2012). Hormonal modulation of plant immunity. Annual Review of Cell and Developmental Biology 28, 489-521.

Pieterse, C. M. J., Leon-Reyes, A., Van der Ent, S. and Van Wees, S. C. M. (2009). Networking by small-molecule hormones in plant immunity. Nature Chemical Biology 5, 308-316.

Prasch, C. M. and Sonnewald, U. (2013). Simultaneous application of heat, drought, and virus to Arabidopsis plants reveals significant shifts in signaling networks. Plant Physiology 162, 1849-66.

Prasch, C. M. and Sonnewald, U. (2015). Signaling events in plants: Stress factors in combination change the picture. Environmental and Experimental Botany 114, 4-14.

Ramegowda, V. and Senthil-Kumar, M. (2015). The interactive effects of simultaneous biotic and abiotic stresses on plants: mechanistic understanding from drought and pathogen combination. Journal of Plant Physiology 176, 47-54.

Ramegowda, V., Senthil-Kumar, M., Ishiga, Y., Kaundal, A., Udayakumar, M. and Mysore, K. S. (2013). Drought Stress Acclimation Imparts Tolerance to Sclerotinia sclerotiorum and Pseudomonas syringae in Nicotiana benthamiana. International Journal of Molecular Sciences 14, 9497-9513.

Rasmussen, S., Barah, P., Suarez-Rodriguez, M. C., Bressendorff, S., Friis, P., Costantino, P., Bones, A. M., Nielsen, H. B. and Mundy, J. (2013a). Transcriptome Responses to Combinations of Stresses in Arabidopsis. Plant Physiology 161, 1783-1794.

Rejeb, I. B., Pastor, V. and Mauch-Mani, B. (2014). Plant responses to simultaneous biotic and abiotic stress: molecular mechanisms. Plants 3, 458-475.

Riera, M., Valon, C., Fenzi, F., Giraudat, J. and Leung, J. (2005). The genetics of adaptive responses to drought stress: abscisic acid-dependent and abscisic acid-independent signalling components. Physiologia Plantarum 123, 111-119.

Rizhsky, L., Davletova, S., Liang, H. and Mittler, R. (2004). The Zinc Finger Protein Zat12 Is Required for Cytosolic Ascorbate Peroxidase 1 Expression during Oxidative Stress in Arabidopsis. Journal of Biological Chemistry 279, 11736-11743.

Robert-Seilaniantz, A., Bari, R. and Jones, J. D. G. (2010). A Biotic or Abiotic Stress? In Abiotic Stress Adaptation in Plants, eds. A. Pareek S. K. Sopory H. J. Bohnert and Govindjee), pp. 103-122: Springer Netherlands.

Robert-Seilaniantz, A., Grant, M. and Jones, J. D. G. (2011). Hormone crosstalk in plant disease and defense: more than just jasmonate-salicylate antagonism. Annual Review of Phytopathology 49, 317-343.

Rowe, H. C., Walley, J. W., Corwin, J., Chan, E. K. F., Dehesh, K. and Kliebenstein, D. J. (2010). Deficiencies in jasmonate-mediated plant defense reveal quantitative variation in Botrytis cinerea pathogenesis. PLoS Pathogens 6, e1000861.

Shaik, R. and Ramakrishna, W. (2013). Genes and co-expression modules common to drought and bacterial stress responses in Arabidopsis and rice. PLOS ONE 8 , e77261.

Shi, H., Wang, X., Ye, T., Chen, F., Deng, J., Yang, P., Zhang, Y. and Chan, Z. (2014). The Cysteine2/Histidine2-Type Transcription Factor ZINC FINGER OF 
ARABIDOPSIS THALIANA6 Modulates Biotic and Abiotic Stress Responses by Activating Salicylic Acid-Related Genes and C-REPEAT-BINDING FACTOR Genes in Arabidopsis. Plant Physiology 165, 1367-1379.

Shinozaki, K. and Yamaguchi-Shinozaki, K. (2007). Gene networks involved in drought stress response and tolerance. Journal of Experimental Botany 58, 221-7.

Shinozaki, K., Yamaguchi-Shinozaki, K. and Seki, M. (2003). Regulatory network of gene expression in the drought and cold stress responses. Current Opinion in Plant Biology 6, 410-417.

Smékalová, V., Doskočilová, A., Komis, G. and Šamaj, J. (2014). Crosstalk between secondary messengers, hormones and MAPK modules during abiotic stress signalling in plants. Biotechnology Advances 32, 2-11.

Soler, R., Erb, M. and Kaplan, I. (2013). Long distance root-shoot signalling in plant-insect community interactions. Trends in Plant Science 18, 149-156.

Stam, J. M., Kroes, A., Li, Y., Gols, R., van Loon, J. J., Poelman, E. H. and Dicke, M. (2014). Plant interactions with multiple insect herbivores: from community to genes. Annu Rev Plant Biol 65, 689-713.

Stein, R. J. and Waters, B. M. (2011). Use of natural variation reveals core genes in the transcriptome of iron-deficient Arabidopsis thaliana roots. Journal of Experimental Botany.

Suzuki, N., Rivero, R. M., Shulaev, V., Blumwald, E. and Mittler, R. (2014). Abiotic and biotic stress combinations. New Phytologis 203, 32-43.

Takahashi, F., Mizoguchi, T., Yoshida, R., Ichimura, K. and Shinozaki, K. (2011). Calmodulin-dependent activation of MAP kinase for ROS homeostasis in Arabidopsis. Molecular Cell 41, 649-60.

Thomma, B. P. H. J., Eggermont, K., Penninckx, I. A. M. A., Mauch-Mani, B., Vogelsang, R., Cammue, B. P. A. and Broekaert, W. F. (1998). Separate jasmonate-dependent and salicylate-dependent defense-response pathways in Arabidopsis are essential for resistance to distinct microbial pathogens. Proceedings of the National Academy of Sciences of the United States of America 95, 15107-15111.

Thomma, B. P. H. J., Eggermont, K., Tierens, K. F. M. and Broekaert, W. F. (1999). Requirement of functional ethylene-insensitive 2 gene for efficient resistance of Arabidopsis to infection by Botrytis cinerea. Plant Physiology 121, 1093-1102.

Tsugama, D., Liu, S. and Takano, T. (2012). Drought-induced activation and rehydration-induced inactivation of MPK6 in Arabidopsis. Biochemical and Biophysical Research Communications 426, 626-629.

Tuteja, N. (2007). Abscisic Acid and abiotic stress signaling. Plant Signal Behav 2, 135-8.

Tuteja, N. and Sarvajeet, S. G. (2012). Plant Acclimation to Environmental Stress: Springer New York.

Vos, I. A., Moritz, L., Pieterse, C. M. J. and Van Wees, S. C. M. (2015). Impact of hormonal crosstalk on plant resistance and fitness under multi-attacker conditions. Frontiers in Plant Science 6, 639.

Vos, I. A., Verhage, A., Schuurink, R. C., Watt, L. G., Pieterse, C. M. J. and Van Wees, S. C. M. (2013). Onset of herbivore-induced resistance in systemic tissue primed for jasmonate-dependent defenses is activated by abscisic acid. Frontiers in Plant Science 4, 539.

Weigel, D. (2012). Natural variation in Arabidopsis: from molecular genetics to ecological genomics. Plant Physiology 158, 2-22.

Wijnen, C. L. and Keurentjes, J. J. B. (2014). Genetic resources for quantitative trait analysis: novelty and efficiency in design from an Arabidopsis perspective. Current Opinion in Plant Biology 18, 103-109.

Xu, P., Chen, F., Mannas, J. P., Feldman, T., Sumner, L. W. and Roossinck, M. J. (2008). Virus infection improves drought tolerance. New Phytologist180, 911-21.

Yamaguchi-Shinozaki, K. and Shinozaki, K. (2005). Organization of cis-acting regulatory elements in osmotic- and cold-stress-responsive promoters. Trends in Plant Science 10, 88-94. 
Yu, L., Nie, J., Cao, C., Jin, Y., Yan, M., Wang, F., Liu, J., Xiao, Y., Liang, Y. and Zhang, W. (2010). Phosphatidic acid mediates salt stress response by regulation of MPK6 in Arabidopsis thaliana. New Phytologist 188, 762-73. 



\section{OHAPTIER}

\section{Genetic architecture of plant stress resistance: multi- trait genome-wide association mapping}

Manus P.M. Thoen ${ }^{1,2,3} \neq$, Nelson H. Davila Olivas ${ }^{1} \neq$, Karen J. Kloth ${ }^{1,2,3} \neq$, Silvia Coolen ${ }^{4} \neq$, Ping-Ping Huang ${ }^{5} \neq$, Mark G.M. Aarts ${ }^{5}$, Johanna A. Bac-Molenaar ${ }^{2,5}$, Jaap Bakker ${ }^{7}$, Harro J. Bouwmeester ${ }^{2}$, Colette Broekgaarden ${ }^{4,9}$, Johan Bucher ${ }^{9}$, Jacqueline Busscher-Lange ${ }^{1,2,3}$, Xi Cheng ${ }^{2}$, Emilie F. Fradin ${ }^{2}$, Maarten A. Jongsma ${ }^{3}$, Magdalena M. Julkowska ${ }^{8,11}$, Joost J.B. Keurentjes ${ }^{5}$, Wilco Ligterink ${ }^{2}$, Corné M.J. Pieterse ${ }^{4}$, Carolien Ruyter-Spira ${ }^{2}$, Geert Smant ${ }^{7}$, Christa Testerink ${ }^{8,11}$, Björn Usadel ${ }^{10}$, Joop. J.A. van Loon ${ }^{1}$, Johan A. van Pelt ${ }^{4}$, Casper C. van Schaik ${ }^{7}$, Saskia C.M. van Wees ${ }^{4}$, Richard G.F. Visser ${ }^{9}$, Roeland Voorrips ${ }^{9}$, Ben Vosman ${ }^{9}$, Dick Vreugdenhil ${ }^{2}$, Sonja Warmerdam ${ }^{7}$, Gerrie L. Wiegers ${ }^{1,3}$, Joost van Heerwaarden ${ }^{6}$, Willem Kruijer ${ }^{6}$, Fred A. van Eeuwijk ${ }^{6}$ \& Marcel Dicke ${ }^{1 *}$

₹ These authors contributed equally to this work.

New Phytologist, accepted

${ }^{1}$ Laboratory of Entomology, Wageningen University, P.O. Box 16, 6700 AA Wageningen, The Netherlands

2 Laboratory of Plant Physiology, Wageningen University, P.O. Box 16, 6700 AA Wageningen, The Netherlands

${ }^{3}$ Business unit Bioscience, Plant Research International, Wageningen UR, P.O. Box 16, 6700 AA Wageningen, The Netherlands

${ }^{4}$ Plant-Microbe Interactions, Department of Biology, Utrecht University, P.O. Box 800.56, 3508 TB, Utrecht, The Netherlands;

${ }^{5}$ Laboratory of Genetics, Wageningen University, P.O. Box 16, 6700 AA Wageningen, The Netherlands

${ }^{6}$ Biometris, Wageningen University and Research centre, P.O. Box 16, 6700 AA Wageningen, The Netherlands

7 Laboratory of Nematology, Wageningen University, P.O. Box 8213, 6700 ES Wageningen, The Netherlands

${ }^{8}$ Section of Plant Physiology, Swammerdam Institute for Life Sciences, University of Amsterdam, P.O. Box 94215, 1090 GE Amsterdam, The Netherlands.

${ }^{9}$ Wageningen UR Plant Breeding, Wageningen University \& Research Centre, P.O. Box 386, 6700 AJ Wageningen, The Netherlands

${ }^{10}$ RWTH Aachen University, Institute for Biology I, Worringer Weg 3, 52074 Aachen, Germany

${ }^{11}$ Section of Plant Cell Biology, Swammerdam Institute for Life Sciences, University of Amsterdam, P.O. Box 94215, 1090 GE Amsterdam, The Netherlands. 


\section{Abstract}

- Plants are exposed to combinations of various biotic and abiotic stresses, but stress responses are usually investigated for single stresses only. Here, we investigated the genetic architecture underlying plant responses to 11 single stresses and several of their combinations by phenotyping 350 Arabidopsis thaliana accessions. A set of $214 \mathrm{k}$ SNPs was screened for marker-trait associations in Genome-Wide Association analyses using tailored multi-trait mixed models.

- Stress responses that share phytohormonal signaling pathways also share genetic architecture underlying these responses. After removing the effects of general robustness, for the 30 most significant SNPs average QTL-effectsizes were larger for dual stresses than single stresses.

- Plants appear to deploy broad-spectrum defensive mechanisms influencing multiple traits in response to combined stresses. Association analyses identified QTLs with contrasting and with similar responses to (a) biotic versus abiotic stresses and (b) belowground versus aboveground stresses.

- Our approach allowed for an unprecedented comprehensive genetic analysis of how plants deal with a wide spectrum of stress conditions. 


\section{Introduction}

In nature, plants face variable environments that impose a wide range of biotic and abiotic stresses. These include e.g. belowground and aboveground stresses, stresses imposed by unicellular and multicellular organisms, short and long-lasting stresses. Under natural conditions, these stresses do not occur in isolation but are commonly present simultaneously (Rizhsky et al., 2004; Bergelson \& Roux, 2010; Mittler \& Blumwald, 2010; Vile et al., 2012; Prasch \& Sonnewald, 2013; Rasmussen et al., 2013; Kissoudis et al., 2014; Rivero et al., 2014; Sewelam et al., 2014; Suzuki et al., 2014). Thus, plants are under strong selection to adapt to local conditions and have evolved sophisticated mechanisms to withstand multiple adverse environmental conditions (Howe \& Jander, 2008; Bergelson \& Roux, 2010; Pieterse et al., 2012; Stam et al., 2014; Brachi et al., 2015; Julkowska \& Testerink, 2015; Kerwin et al., 2015). Yet, investigating this in a targeted experimental way is a major challenge due to the complexity of multiple stress exposure. To gain insight into the adaptation of plants to the wide variety of stress-inducing conditions they face, genetic variation and mechanisms underlying stress resistance should be studied (Alonso-Blanco et al., 2009; Brachi et al., 2015; Kerwin et al., 2015). The responses of plants to stresses have traditionally been investigated for individual stresses (Howe \& Jander, 2008), but the research focus is currently shifting towards plant responses to combinations of stresses (Holopainen \& Gershenzon, 2010; Pierik \& Testerink, 2014; Stam et al., 2014; Suzuki et al., 2014; Kissoudis et al., 2015). The emerging picture is that responses to stress combinations cannot be predicted reliably from the responses to individual stresses (De Vos et al., 2006; Makumburage et al., 2013). For instance, the majority of transcriptional responses of Arabidopsis to combinations of two abiotic stresses could not be predicted from responses to the individual stresses (Rasmussen et al., 2013). Moreover, phenotype expression in response to two biotic stresses could not be predicted on the basis of existing information on interactions between underlying signaling pathways (De Vos et al., 2006). Phytohormones are major players in a signaling network, mediating responses to both biotic and abiotic stresses (Pieterse et al., 2009). For instance, chewing insect herbivores elicit especially the jasmonic acid (JA), abscisic acid (ABA) and ethylene (ET) signaling pathways, phloem-sucking insects and biotrophic microbial pathogens 
elicit especially the salicylic acid (SA) pathway, and drought elicits the abscisic acid (ABA) pathway (Pieterse et al., 2009). The phytohormonal responses exhibit extensive crosstalk, resulting in specific changes in plant phenotype in response to individual stresses (De Vos et al., 2005; Pieterse et al., 2012).

In plant breeding, resistance and tolerance to multiple stresses is a common selection target (Braun et al., 1996). A well-known strategy to achieve resistance and tolerance is by evaluation of candidate varieties in multienvironment trials, i.e., field trials at multiple locations during several years (van Eeuwijk et al., 2010; Malosetti et al., 2013). In such trials, multiple stresses can occur, but their occurrence and the intensity with which they occur is not guaranteed and, therefore, plant breeders developed the concept of managed stress trials in which specific and well-defined stress conditions are imposed for a single or a small number of stresses (Cooper \& Hammer, 1996; Cooper et al., 2014). Recently, the urge to manage environmental factors even more precisely has led to the development of phenotyping platforms, where, again, mainly single stresses are investigated (Fiorani \& Schurr, 2013; Granier \& Vile, 2014; Kloth et al., 2015).

Most studies, outside plant breeding, that examined plant responses to multiple stresses included only one or a few genotypes (Holopainen \& Gershenzon, 2010; Rasmussen et al., 2013; Pierik \& Testerink, 2014; Stam et al., 2014; Suzuki et al., 2014; Kissoudis et al., 2015). To obtain a further understanding of the genetic architecture of complex traits such as plant adaptation to a diversity of stresses, extensive study of the natural genetic variation within a species is instrumental. Genome-wide association (GWA) analysis is an important tool for this, requiring a large number of well-genotyped plant accessions. Yet, although the interest in natural variation and GWA mapping is rapidly increasing (Wijnen \& Keurentjes, 2014; Ogura \& Busch, 2015), a large-scale evaluation of natural genetic variation for resistance of plants to the full diversity of stresses that they are exposed to, including pathogens, herbivores and abiotic stresses and their interactions, has not been made to date. To elucidate the genetic architecture of plant stress resistance, an integrated approach is needed that models the genetics of responses to a range of single and combined stresses, including the interaction between those responses. Here, we have taken a comprehensive and integrated approach to 
investigate the genetics underlying plant responses to 15 carefully standardized single stresses or stress combinations (Table 1), making use of a global population of 350 Arabidopsis accessions that have been genotyped for $214 \mathrm{k}$ SNPs (Baxter et al., 2010; Li et al., 2010). The standardization of these 15 stress conditions is an important element of the study because it allows for phenotyping of well-defined stress responses. We developed a tailored multitrait GWA analysis that allowed the identification of candidate genes associated with plant responses to multiple stresses that were validated by gene expression and mutant analyses. 


\section{Materials and Methods}

\section{Arabidopsis thaliana population}

In this study we included 350 Arabidopsis thaliana (L.) Heynh. accessions from the Hapmap population (http://bergelson.uchicago.edu/wpcontent/uploads/2015/04/Justins-360-lines.xls). The Hapmap population has been genotyped for 250K bi-allelic SNPs (Baxter et al., 2010; Platt et al., 2010; Chao et al., 2012) and after quality control and imputation this SNP-set was reduced to a set of 214,051 SNPs.

\section{Definition of the target traits}

For every experiment, the target traits were derived from the individual plant data using the following strategy. First, when residuals deviated from normality, a logarithmic, arcsine or square root transformation was applied to the original observations. Second, genotypic (accession) means for each treatment were calculated using a mixed model to account for design effects. Different mixed models were used in the experiments, reflecting the different designs. In all cases, accession effects were modelled as fixed, and the accession means were the best linear unbiased estimator (BLUE) of these effects. Third, for traits measured in treatment and control conditions, differences or residuals (when regressing treatment on control values) were defined, in order to obtain a measure of stress tolerance that was corrected for the expression of the same trait under control conditions. Finally, within each experiment, the traits were replaced by the first principal component if the latter explained more than half of the variation in all traits in this experiment; in all other cases the original traits were retained. An overview of final traits and their corresponding sections in the Supplementary Methods can be found in Table 1 . In case of replacement by the first principal component, original traits and the variance explained by the first principal component are listed (Supplementary Methods Tables M1-M5). In total, phenotypic data for 73 individual traits were obtained by 10 different research groups. All calculations were performed in $\mathrm{R}$, unless stated otherwise. Mixed model analysis was performed with the R-package ASReml (Butler et al., 2009). In all equations the term $E$ denotes residual error. 
All other terms represent fixed effects unless stated otherwise. A colon $(:)$ is used to define interactions between terms.

\section{Statistics}

\section{Genetic correlation networks and heritability}

Pairwise marker-based genetic correlations between traits, genomic correlations, were estimated using a multi-trait mixed model (MTMM) (Korte et al., 2012). Residuals were assumed uncorrelated for traits that were measured on different plants. For some pairs of traits the likelihood was monotone, which can also occur in single-trait mixed models (Kruijer et al., 2015). In this case, the genetic correlation was estimated by the (Pearson) correlation between the univariate G-BLUPs (De los Campos et al., 2013) estimated for these traits. A network between predefined groups of traits was constructed by connecting groups whose average genetic correlation across pairs of traits was above 0.2 .

Narrow sense heritability (Table S1) was estimated using the mixed model $Y_{i}=\mu+A_{i}+E_{i} \quad$ where $Y_{i}$ represents the phenotypic means of accessions $(i=1, \ldots, 350)$, and $A_{i}$ and $E_{i}$ random genetic and residual effects. The vector of additive genetic effects follows a multivariate normal distribution with covariance $\sigma_{A}^{2} K, K$ being a marker-based relatedness matrix. The residual errors are independent, with variance $\sigma_{E}^{2}$. We obtained REML-estimates of $\sigma_{A}^{2}$ and $\sigma_{E}^{2}$, and estimated heritability as $h^{2}=\sigma_{A}^{2} /\left(\sigma_{A}^{2}+\sigma_{E}^{2}\right)$. This is an estimate of narrow-sense heritability, since the model for the genetic effects only captures additive effects, and $\sigma_{E}^{2}$ is the sum of environmental and non-additive genetic effects (see e.g. Kruijer et al. (2015)).

\section{Multi-trait mixed models}

Following Zhou and Stephens (2014), we assume the MTMM $Y=X B+G+E$, with $Y$ being the genotypes by traits $(n \times p)$ matrix of phenotypic observations. The terms $X B, G$ and $E$ stand for respectively the fixed effects (including trait specific intercepts and SNP-effects) and the random genetic and environmental effects. $G$ follows a zero mean matrix-variate normal distribution with rowcovariance (marker-based kinship) matrix $K$ and column (trait) covariance matrix $V_{g} . V_{g}$ is a $p \times p$ matrix modeling the genetic correlations between traits. 
This is equivalent with $g=\operatorname{vec}(G)$ (the vector containing the columns of $G$ being multivariate normal with a covariance matrix defined by the Kronecker product $V_{g} \otimes K$ (Zhou \& Stephens, 2014). Similarly, vec(E) follows a zero mean normal distribution with covariance $V_{e} \otimes I_{n}$, where $V_{e}$ accounts for the non-genetic correlations between traits.

\section{Factor-analytic models}

Since $V_{g}$ and $V_{e}$ contain a total of $p(p+1)$ parameters, the MTMM above becomes difficult to fit for more than 10 traits (Zhou \& Stephens, 2014). For $V_{g}$ we therefore assumed a factor analytic model, which is well known in the context of QTL-mapping for experimental populations with limited numbers of markers (Boer et al., 2007), but has not been used in the context of multivariate GWAS. As almost all traits were derived from measurements on different plants, a diagonal model $V_{e}=\operatorname{diag}\left(\sigma_{e, 1}^{2}, \ldots, \sigma_{e, p}^{2}\right)$ was chosen for the environmental covariances. For $V_{g}$ a second order factor analytic structure was chosen $V_{g}=$ $\sigma_{g}^{2}\left(\lambda \lambda^{t}+\operatorname{diag}\left(\tau_{1}^{2}, \ldots, \tau_{p}^{2}\right)\right)$, where $\sigma_{g}^{2}$ represents a scale parameter, the magnitude of genetic effects, the $p \times 2$ matrix $\lambda$ contains the trait specific scores belonging to the factor analytic part of the model that provides a rank two variancecovariance structure between traits, and $\operatorname{diag}\left(\tau_{1}^{2}, \ldots, \tau_{p}^{2}\right)$ provides trait specific residual genetic variances (Piepho, 1997; Meijer, 2009). The model was fitted with the R-package ASRreml (Butler et al., 2009).

\section{Compressed kinship}

Factor analytic models have been successfully applied to experimental populations with a simple genetic relatedness structure (Boer et al., 2007; Malosetti et al., 2008; Alimi et al., 2013), but currently available software could not perform REML-estimation for the Hapmap-population. The kinship matrix was therefore replaced by a compressed kinship matrix (Bradbury et al., 2007; Zhang et al., 2010), modeling the genetic relatedness between a number of internally homogeneous groups. Assuming there are $m$ such groups, containing $n_{1}, \ldots, n_{m}$ accessions each, the original kinship matrix $K$ is replaced by $Z K_{C} Z^{t}$, where $K_{C}$ is the kinship matrix for the groups, and $Z$ is the $n \times m$ incidence matrix assigning each of the $n$ accessions to one of the $m$ groups. The groups were created by a procedure that restricted the marker data to be linear 
combinations of environmental covariates representing the conditions at the place of origin of the accessions, as explained below.

Compressed kinship was calculated as the average kinship within genetic groups. Genotypes were assigned to $k$ genetic groups by performing Ward clustering based on the squared Euclidean distance along the first $k-1$ principal components calculated from a matrix of standardized SNP scores, followed by cutting the resulting dendrogram into $k$ distinct clusters (van Heerwaarden et al., 2012; Odong et al., 2013; van Heerwaarden et al., 2013).

The use of a compressed kinship matrix requires a choice of the level of compression, as determined by the number of genetic groups over which the individual kinship is averaged. This choice needs to balance the gain in computational efficiency with model fit (Zhang et al., 2010) and the ability of the compressed matrix to capture the correlation between genetic dissimilarity and phenotypic differences, which is ultimately the reason for including a kinship matrix in the association model. There are currently no standard methods to determine the optimum level of compression, at least not when used in a multitrait setting. We determined the appropriate level of compression for each association model based on the model likelihood, convergence and correspondence between kinship and phenotypic and geographical similarity. The latter was quantified as the Frobenius norm of the difference between the complement of the compressed kinship matrix, expanded to a block matrix of full rank, and the Euclidean distance matrix of phenotypic traits or geographic coordinates. We considered a range of 4 to 100 groups. Correspondence with phenotypic and geographical dissimilarity increased steeply from 4 to around 35 groups, after which correspondence with geographic distance increased more slowly and the correspondence with phenotypic distance showing a local decrease until 58 groups. Model likelihood was relatively stable above 4 groups but convergence was erratic depending on the modeled contrasts. For each model the number of groups was therefore chosen to be the minimum number of groups needed to achieve a level of correspondence approximating that found at 35 groups, under condition of model convergence. 
Traits (columns of $Y$ ) were standardized. Along the genome, MTMMs of the type $Y=X B+G+E$ were fitted with initially for each marker trait-specific QTL effects $\beta_{1}, \ldots, \beta_{p}$ (contained in $B$ ). To identify general QTLs with trait-specific effects, for individual markers, the null hypothesis $\beta_{1}=\beta_{2}=\cdots \beta_{p}=0$ was tested by a Wald test against the alternative hypothesis that at least one of the trait specific effects was nonzero (Zhou \& Stephens, 2014). To identify consistent QTLs, the null hypothesis $\beta_{1}=\beta_{2}=\cdots \beta_{p}=\beta \neq 0$ was tested. To identify potentially adaptive QTLs, contrasts defined on the trait specific QTL effects were tested. For example, suppose the first $p 1$ of the full set of $p$ traits represent responses measured under abiotic stresses, while the second $p 2$ traits represent responses under biotic stresses. A contrast can now be defined to test the hypothesis whether the QTL effect for abiotic stresses differs from that for biotic stresses: $\beta_{1}=\beta_{2}=\cdots \beta_{p 1}=\alpha_{\text {abiotic }} ; \beta_{p 1+1}=\beta_{p 1+2}=\cdots \beta_{p}=\alpha_{\text {biotic }}$ and $H_{0}: \alpha_{\text {abiotic }}=\alpha_{\text {biotic }}$ versus $H_{a}: \alpha_{\text {abiotic }} \neq \alpha_{\text {biotic }}$. For the Wald test for the hypothesis $\beta_{1}=\ldots=\beta_{p}$ we first fit the MTMM $Y=X B+G+E$ with $X B$ only containing trait specific means $\mu_{1}, \ldots, \mu_{p}$, and next test hypotheses on the marker effects. The contrast is defined through a partitioning of the traits in two groups (e.g. resistance against biotic or abiotic stress). Using the R-package asreml (Butler et al., 2009) we perform Wald tests for the following hypotheses:

1. $H_{0}: \beta=0$, in the constrained model $\beta_{1}=\ldots=\beta_{p}=\beta$.

2. $H_{0}: a_{1}=a_{2}$, in the constrained model where $a_{1}$ is the effect on all traits in the first group, and $a_{2}$ for traits in the second group.

Simulations to compare power for full MTMM, contrast MTMM and univariate analysis

We further compared the different Wald tests using simulations, described in more detail in the Supplementary Methods (SM.12). Specifically, we compared the performance of the general MTMM (i.e. testing the hypothesis $\beta_{1}=\beta_{2}=$ $\cdots \beta_{p}=0$ ) with the MTMM used for the contrasts ( i.e. $H_{0}: \alpha_{\text {group } 1}=\alpha_{\text {group } 2}$, where, within two predefined groups of traits, all SNP-effects equal $\alpha_{\text {group } 1}$ respectively $\alpha_{\text {group } 2}$ ). We simulated phenotypic data for given genotypic data, either assuming SNP-effects positive (but not equal) within one group of traits and negative for the other (Scenario A), or the sign of each SNP effect being chosen randomly (Scenario $B$ ). The simulation results as presented in Fig. S11 
(see later) clearly indicate that the Wald test for the contrast has superior power under scenario A, while the general MTMM performs best under scenario $B$. In both cases, univariate analysis of the trait with the highest heritability is outperformed by at least one of the MTMM analyses. As a consequence, univariate GWAS and GWAS with the general and contrast MTMM give different rankings of SNPS.

\section{Selecting candidate genes}

A significance threshold of $P<0.0001$ was chosen after implementation of genomic control (see below). For MTMM this resulted in 43 SNPs meeting this criterion. Such a threshold of 0.0001 is not uncommon in studies involving single trait GWAS (e.g., El Soda et al. 2015; van Rooijen et al. 2015; Kooke et al. 2016). Given the total number of analysed SNPs (i.e. 199589 SNPs having a minor allele frequency above 0.05) and under the null hypothesis of no QTLs and independence of the markers, we arrive at a naive estimate for the expected number of false positives of around 20, which is considerably smaller than the 43 SNPs with $P<0.0001$ recorded in the full MTMM, suggesting that about half of the significant SNPs must be true positives. Furthermore, following the procedure described by Benjamini and Hochberg (1995), we estimated the false discovery rate to be 0.45 , a number very comparable to our naive estimate above. SNPs within a $20 \mathrm{~kb}$ region were considered to be part of one LD block. This resulted in 30 genomic regions. For presentation purposes, each LD block was represented in Figures and heatmaps by the SNP with the on average strongest (absolute) effect across all traits. For the GWA contrast analyses, the same procedure was followed to define LD blocks and representative SNPs. 


\section{Correcting for genomic inflation}

The Wald test is known to suffer from some inflation (Zhou \& Stephens, 2014), which we correct for by using genomic control (GC) (Devlin \& Roeder, 1999; Devlin et al., 2001), which divides the observed test statistics $T_{1}, \ldots, T_{p}$ by the genomic inflation factor. For both the unconstrained MTMM and the MTMM for contrasts described above, we observed inflation for small as well as large $P$ values (i.e. also more $P$-values close to one than expected). Consequently, the usual genomic control procedures based on the observed versus expected median of test statistics gave too optimistic inflation factors. We therefore applied an alternative genomic control procedure, in which we regress the observed $-\log _{10}(P)$ values on the expected ones, and correct the observed $-\log _{10}(P)$ values for the slope. The genomic inflation factor was 1.24 for the full MTMM, with similar values for the other MTMM analyses (between 1.07 and 1.38). For the full MTMM without correction for population structure (i.e. taking the kinship to be the identity matrix), the inflation factor was 2.36 . 


\section{Results}

The phenotypic response of a population of 350 Arabidopsis accessions to an extensive set of stress-inducing conditions was quantified relative to the respective control treatments. Correcting for the respective control means that in the residual signal for a trait, effects of earliness, flowering time, general robustness, vigour, etc., have been removed already. Therefore, the traits as analysed represent a kind of stress per se response from which all kinds of disturbances have already been eliminated. Thirty traits, including e.g. root length, number of damaged leaves, or number of pathogen-inflicted spreading lesions (Table 1) were quantified when the plants were exposed to 15 different stresses, i.e. four abiotic stresses (drought, salt stress, osmotic stress and heat), seven biotic stresses (parasitic plant, phloem-feeding aphid, phloem-feeding whitefly, cell-content feeding thrips, leaf-chewing caterpillar, root-feeding nematode, and necrotrophic fungus) and four stress combinations (fungus and caterpillar, drought and fungus, drought and caterpillar, caterpillar and osmotic stress). For detailed information on the carefully standardized stress treatments, the trait definitions and phenotyping, see Supplementary Methods. 
Table 1. Phenotypes assessed. The dataset contains three plant stress categories applied to Arabidopsis thaliana; abiotic stress, biotic stress and combinations of both abiotic and biotic stress. Phenotype assessments that were performed under similar environmental conditions have similar background shading (light and dark grey). 'Phenotype' refers to different phenotypic assessments (in some cases the first principal component of a group of phenotypes). 'Treatment' refers to the sort of stress that was applied. Additional information on traits can be found in Supplementary Methods. Yellow indicates abiotic stress, green indicates biotic stress and blue indicates combinations of biotic and abiotic stress.

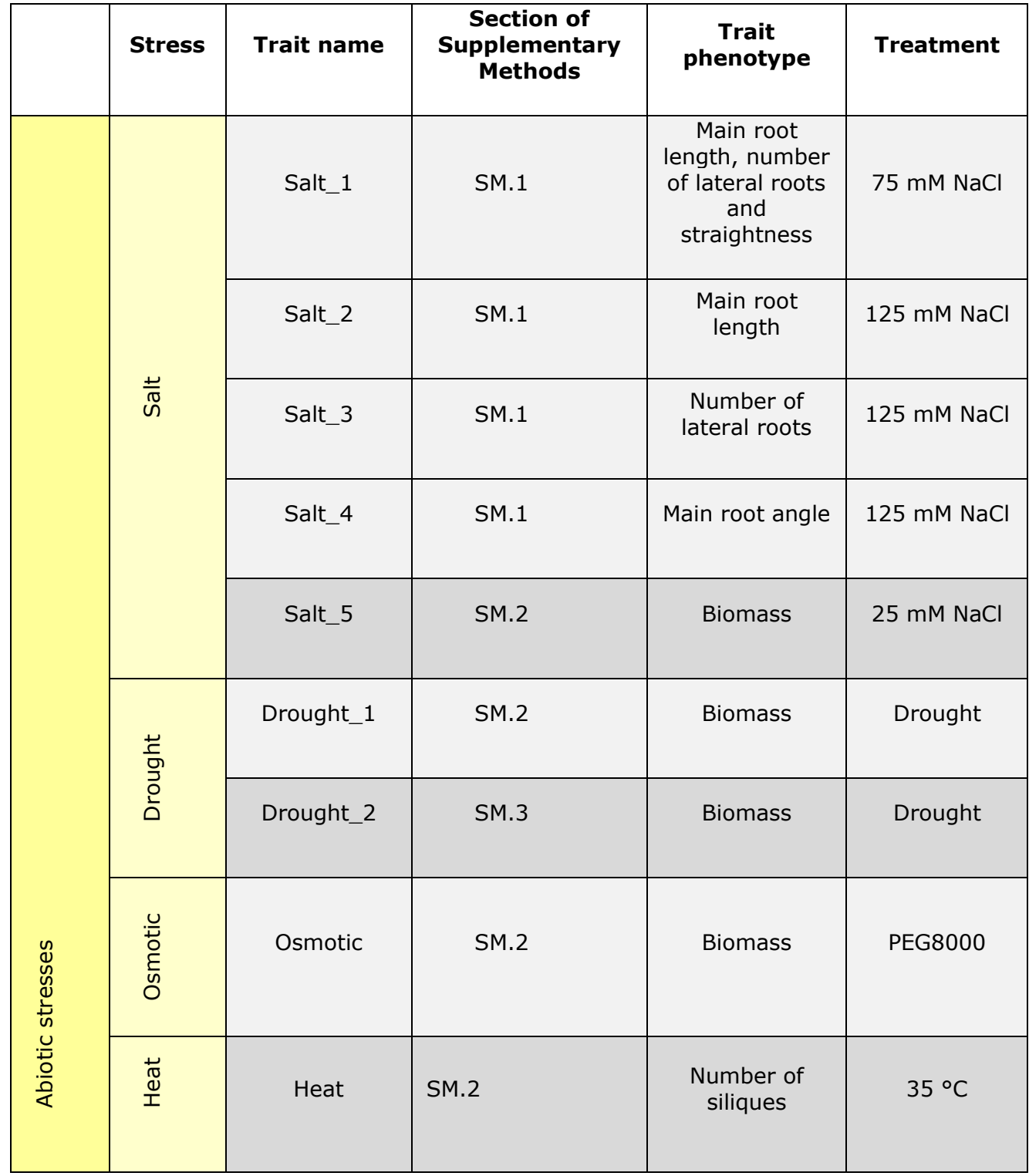




\begin{tabular}{|c|c|c|c|c|c|}
\hline \multirow{13}{*}{ 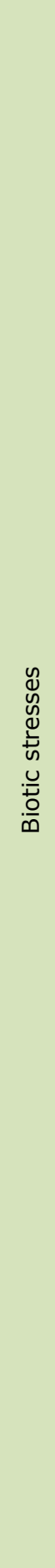 } & 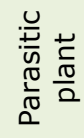 & Parasitic plant & SM.4 & Attachments & $\begin{array}{c}\text { Phelipanche } \\
\text { ramosa }\end{array}$ \\
\hline & 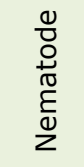 & Nematode & SM. 5 & $\begin{array}{l}\text { Offspring, } \\
\text { eggmass }\end{array}$ & $\begin{array}{c}\text { Meloidogyne } \\
\text { incognita }\end{array}$ \\
\hline & \multirow{2}{*}{ 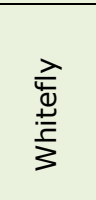 } & Whitefly_1 & SM. 6 & $\begin{array}{l}\text { Survival, } \\
\text { whiteflies }\end{array}$ & $\begin{array}{l}\text { Aleyrodes } \\
\text { proletella }\end{array}$ \\
\hline & & Whitefly_2 & SM. 6 & $\begin{array}{c}\text { Reproduction, } \\
\text { eggs }\end{array}$ & A. proletella \\
\hline & \multirow{3}{*}{$\frac{\text { 므 }}{\frac{1}{2}}$} & Aphid_1 & SM.7 & $\begin{array}{l}\text { Behavior T1, } \\
\text { probing }\end{array}$ & $\begin{array}{l}\text { Myzus } \\
\text { persicaee }\end{array}$ \\
\hline & & Aphid_2 & SM.7 & $\begin{array}{c}\text { Behavior T2, } \\
\text { probing }\end{array}$ & M. persicae \\
\hline & & Aphid_3 & SM.7 & $\begin{array}{l}\text { Offspring, } \\
\text { aphids }\end{array}$ & M. persicae \\
\hline & \multirow{3}{*}{$\frac{\text { Un }}{\frac{n}{E}}$} & Thrips_1 & SM. 8 & $\begin{array}{l}\text { Feeding } \\
\text { damage }\end{array}$ & $\begin{array}{l}\text { Frankliniella } \\
\text { occidentalis }\end{array}$ \\
\hline & & Thrips_2 & SM. 8 & Behavior T1 & $\begin{array}{c}\text { F. } \\
\text { occidentalis }\end{array}$ \\
\hline & & Thrips_3 & SM. 8 & Behavior T2 & $\begin{array}{c}F . \\
\text { occidentalis }\end{array}$ \\
\hline & \multirow{3}{*}{ 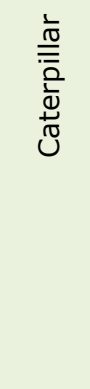 } & Caterpillar_1 & SM.9 & $\begin{array}{l}\text { Leaf area } \\
\text { consumed }\end{array}$ & Pieris rapae \\
\hline & & Caterpillar_2 & SM. 3 & Biomass & P. rapae \\
\hline & & Caterpillar_3 & SM. 3 & $\begin{array}{l}\text { Number of } \\
\text { damaged } \\
\text { leaves and } \\
\text { feeding sites }\end{array}$ & P. rapae \\
\hline
\end{tabular}




\begin{tabular}{|c|c|c|c|c|c|}
\hline & 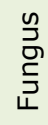 & Fungus & SM.10 & $\begin{array}{l}\text { Number of } \\
\text { spreading } \\
\text { lesions }\end{array}$ & $\begin{array}{l}\text { Botrytis } \\
\text { cinerea }\end{array}$ \\
\hline & $\stackrel{\mathscr{D}}{\underline{D}}$ & $\begin{array}{l}\text { Fungus and } \\
\text { caterpillar_1 }\end{array}$ & SM. 3 & Biomass & $\begin{array}{l}\text { B. cinerea } \\
\text { and } P \text {. rapae }\end{array}$ \\
\hline & $\begin{array}{l}\frac{0}{0} \\
\frac{3}{0} \\
0\end{array}$ & $\begin{array}{l}\text { Fungus and } \\
\text { caterpillar_2 }\end{array}$ & SM. 3 & $\begin{array}{l}\text { Number of } \\
\text { damaged } \\
\text { leaves and } \\
\text { feeding sites }\end{array}$ & $\begin{array}{l}\text { B. cinerea } \\
\text { and } P \text {. rapae }\end{array}$ \\
\hline & & $\begin{array}{l}\text { Caterpillar } \\
\text { and fungus }\end{array}$ & SM.10 & $\begin{array}{l}\text { Number of } \\
\text { spreading } \\
\text { lesions }\end{array}$ & $\begin{array}{l}\text { P. rapae and } \\
B \text {. cinerea }\end{array}$ \\
\hline & 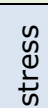 & $\begin{array}{l}\text { Drought and } \\
\text { fungus }\end{array}$ & SM.10 & $\begin{array}{l}\text { Number of } \\
\text { spreading } \\
\text { lesions }\end{array}$ & $\begin{array}{c}\text { Drought and } \\
\text { B. cinerea }\end{array}$ \\
\hline 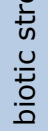 & 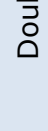 & $\begin{array}{l}\text { Drought and } \\
\text { caterpillar }\end{array}$ & SM. 3 & $\begin{array}{l}\text { Number of } \\
\text { damaged } \\
\text { leaves and } \\
\text { feeding sites }\end{array}$ & $\begin{array}{c}\text { Drought and } \\
\text { P. rapae }\end{array}$ \\
\hline & & $\begin{array}{l}\text { Caterpillar } \\
\text { and } \\
\text { osmotic_1 }\end{array}$ & SM. 9 & $\begin{array}{c}\text { Projected leaf } \\
\text { area }\end{array}$ & $\begin{array}{l}\text { P. rapae and } \\
\text { PEG8000 }\end{array}$ \\
\hline & & $\begin{array}{l}\text { Caterpillar } \\
\text { and } \\
\text { osmotic_2 }\end{array}$ & SM.9 & Biomass & $\begin{array}{l}\text { P. rapae and } \\
\text { PEG } 8000\end{array}$ \\
\hline
\end{tabular}

\section{Heritability of responses to biotic and abiotic stresses}

The phenotypic analysis resulted in a wide range of marker-based narrow sense heritability (Kruijer et al., 2015) estimates with 15 traits of low $\left(h^{2}<0.2\right)$, 10 of moderate $\left(0.2<h^{2}<0.5\right)$ and 5 of high $\left(h^{2}>0.5\right)$ heritability (Figure S1). The number of abiotic stress traits per heritability category was similar, while the number of traits related to biotic and combined stresses decreased with increasing heritability class. The most heritable traits were responses to feeding damage by thrips (Thrips_ $\left.1 ; h^{2}=0.8\right)$, and nematodes $\left(h^{2}=0.7\right)$, and responses to salt (Salt_1 and Salt_3; resp. $h^{2}=0.6$ and $h^{2}=0.7$ ) and heat (Heat; $h^{2}=0.6$ ) (Table S1). The traits related to combined stresses have predominantly low 
heritabilities; however, it should be emphasized that the combined stresses especially relate to combinations involving fungal and caterpillar stress.

\section{Genetic commonality underlying responses to different stresses}

To analyze the phenotypic variation between Arabidopsis accessions as a function of molecular marker variation, we used various mixed model approaches (see Methods section). We estimated marker-based genetic correlations, i.e. correlations based on the genome-wide commonality of SNP effects underlying pairs of traits (see Methods), to investigate the magnitude of genetic commonality underlying resistance mechanisms in response to a range of biotic and abiotic stresses. For brevity, we will refer to these marker-based genetic correlations as genetic correlations. Such genetic correlations can be interpreted as upper boundaries to the joint determination of pairs of traits by genetic factors. Genetic correlation analysis revealed a strong connection between the responses to parasitic plants and to aphids $(r=0.8)$, which were both negatively associated with other stress responses (Figure 1). Parasitic plants and aphids have in common that they target phloem and xylem tissue (Tjallingii \& Hogen Esch, 1993; Dorr \& Kollmann, 1995), and induce the SA phytohormonal pathway (De Vos et al., 2005; Runyon et al., 2008). In contrast, the biotic stress responses that were negatively associated with the responses to parasitic plants and aphids, i.e. responses to necrotrophic fungi, caterpillars, and thrips, represent JA-inducing stresses (De Vos et al., 2005; Pieterse et al., 2009; Pieterse et al., 2012). Because the SA and JA pathways predominantly interact through negative crosstalk (Pieterse et al., 2009), the two main clusters resulting from the genetic correlation analysis represent different phytohormonal signaling response mechanisms. We also observed a strong genetic correlation between plant responses to osmotic stress and root-feeding nematodes. This supports the notion that root-knot nematodes trigger a differentiation of root cells to multinucleate giant cells with severely altered water potential and osmotic pressure (Baldacci-Cresp et al., 2015). While the correlations between traits at the phenotypic level were generally rather low, the genetic correlation analysis revealed a common genetic basis underlying the responses to sets of single and combined stresses (Figure S2). 


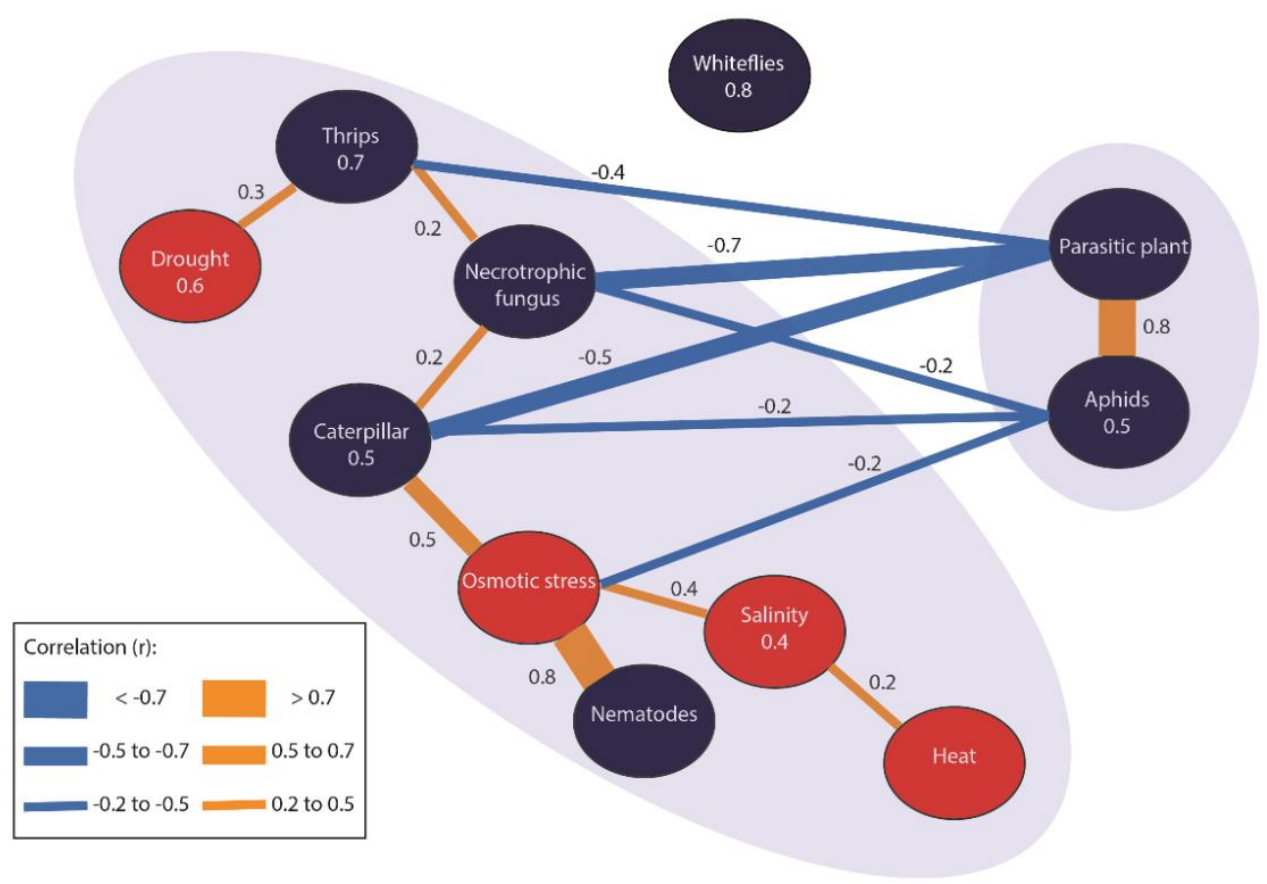

Figure 1. Mean genetic correlations between responses of Arabidopsis thaliana to abiotic (red) and biotic (dark blue) plant stresses. Thickness of lines represents the strength of mean genome-wide correlations, annotated with $r$ values (orange=positive, blue=negative correlation). The more shared genetic associations between stresses, the higher the absolute genetic correlation. Correlations are negative when alleles have opposite effects, i.e. resulting in increased resistance to one stress, but decreased resistance to the other stress. Values in balloons represent mean within-group correlation (not shown for groups consisting of a single trait). Mean between-group correlations are not shown if they are below an absolute value of $r=0.2$. Two clusters can be distinguished: (1) parasitic plants and aphids and (2) the other stresses, except whiteflies. 


\section{Candidate genes underlying responses to stresses}

To identify individual candidate genes that contributed most to the pattern of genetic correlations, we fitted multi-trait QTL mixed models (MTMMs) to the total set of 30 traits, using a $214 \mathrm{k}$ SNP set that is commonly used for GWA studies in Arabidopsis (Kim et al., 2007; Atwell et al., 2010; Li et al., 2010; Horton et al., 2012; Bac-Molenaar et al., 2015). Our multi-trait GWA approach closely follows the modeling framework developed by Zhou and Stephens (2014) and generalizes the use of MTMMs as described previously (Boer et al., 2007; Malosetti et al., 2008; Alimi et al., 2013) for classical biparental offspring populations to association panels. This GWA analysis identified 30 chromosome regions with multiple, significant SNP-trait associations. From each of those regions, the significant SNP with the strongest effect was chosen to represent the locus (Figure 2; Table S2). Clustering of stresses by estimated SNP-effect profiles (Figure 2) indicates that multiple SNPs were associated with response to more than one stress. Stress combinations induced large QTL allele substitution effects in the MTMM mapping (Figure 2 and Table S2), indicating that combinations of stresses trigger broad-spectrum defensive mechanisms. A total of 125 genes were in linkage disequilibrium (LD) with the 30 most significant SNPs from the GWA analysis. Twenty of these genes were stress-related according to gene ontology (GO) annotation data (Table S3). Of these 20 genes, six have been functionally characterized by at least one study (Table 2a). For these six genes, we explored expression data to evaluate the biological relevance of these genes in stress-responsive mechanisms of Arabidopsis (Figure S3). Of special interest were SNPs chr5.7493620, chr5.22041081 and chr4.6805259, that were in LD with WRKY38 (encoding a WRKY transcription factor involved in SA-dependent disease resistance) (Kim et al., 2008), AtCNGC4 (involved in pathogen resistance) (Chin et al., 2013) and RMG1 (coding for disease resistance protein) (Yu et al., 2013) respectively. 


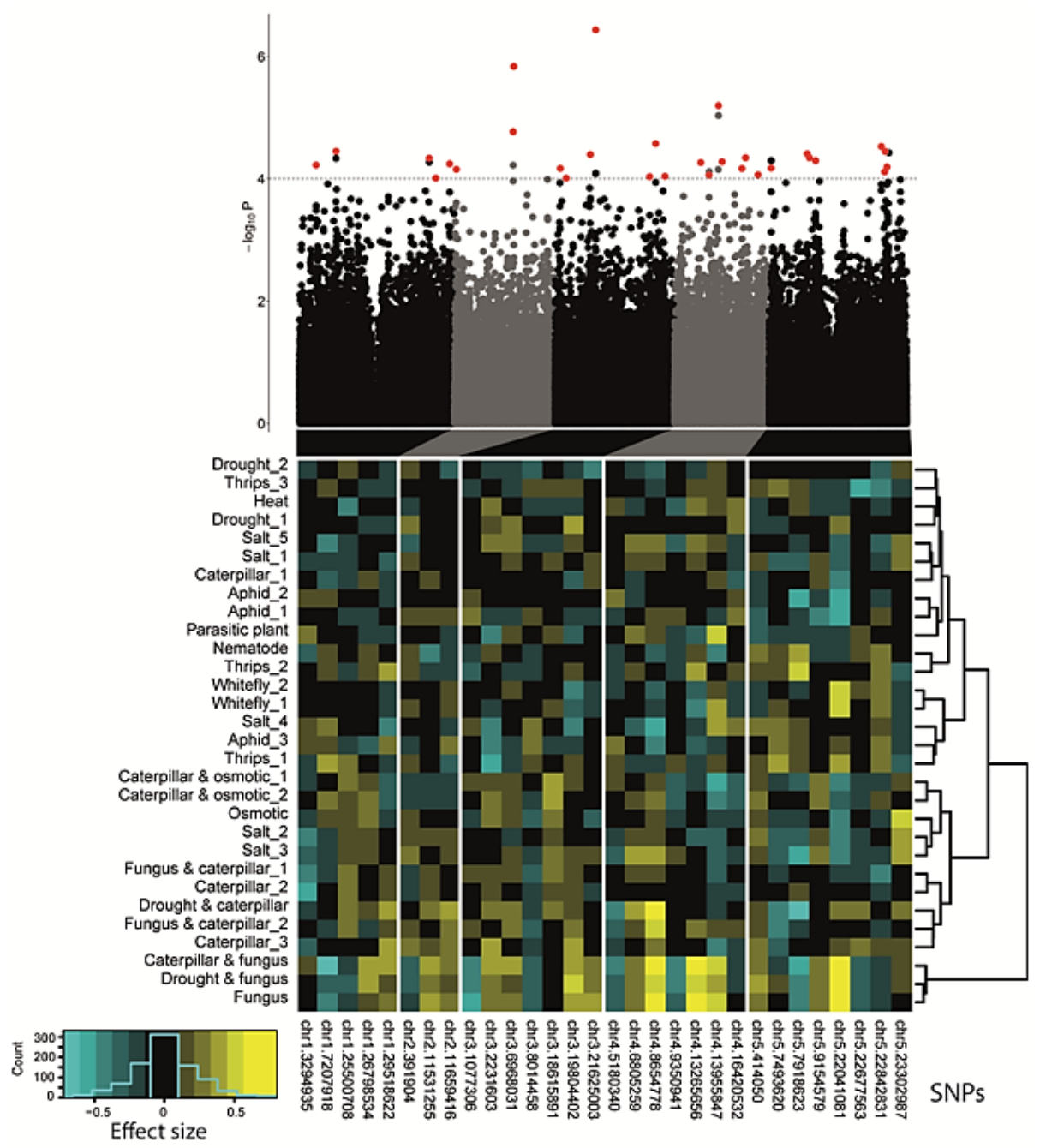

Figure 2. Multi-trait mixed-model (MTMM) GWA mapping with 30 different stress responses of Arabidopsis thaliana. The top panel shows the $214 \mathrm{k}$ SNPs with their corresponding $-\log _{10}(P)$ values for the five chromosomes. The lower panel depicts the traitspecific effect sizes of the rare alleles for significant SNPs $(P<0.0001)$ as estimated by the full MTMM. When several SNPs were located within the $20 \mathrm{~kb}$ linkage disequilibrium halfwindows around the most significant SNP in a region, the effects for the SNP with the on average strongest absolute effects are shown (red-flagged in the Manhattan plot). SNPs are named by chromosome number and position on the chromosome. Negative effect sizes (blue) correspond to reduced plant resistance due to the rare allele, positive effect sizes (yellow) to increased resistance due to the rare allele. Stress responses were clustered hierarchically according to their effect, using Ward's minimum variance method. The key shows the frequency distribution for the effect sizes of the SNPs. 


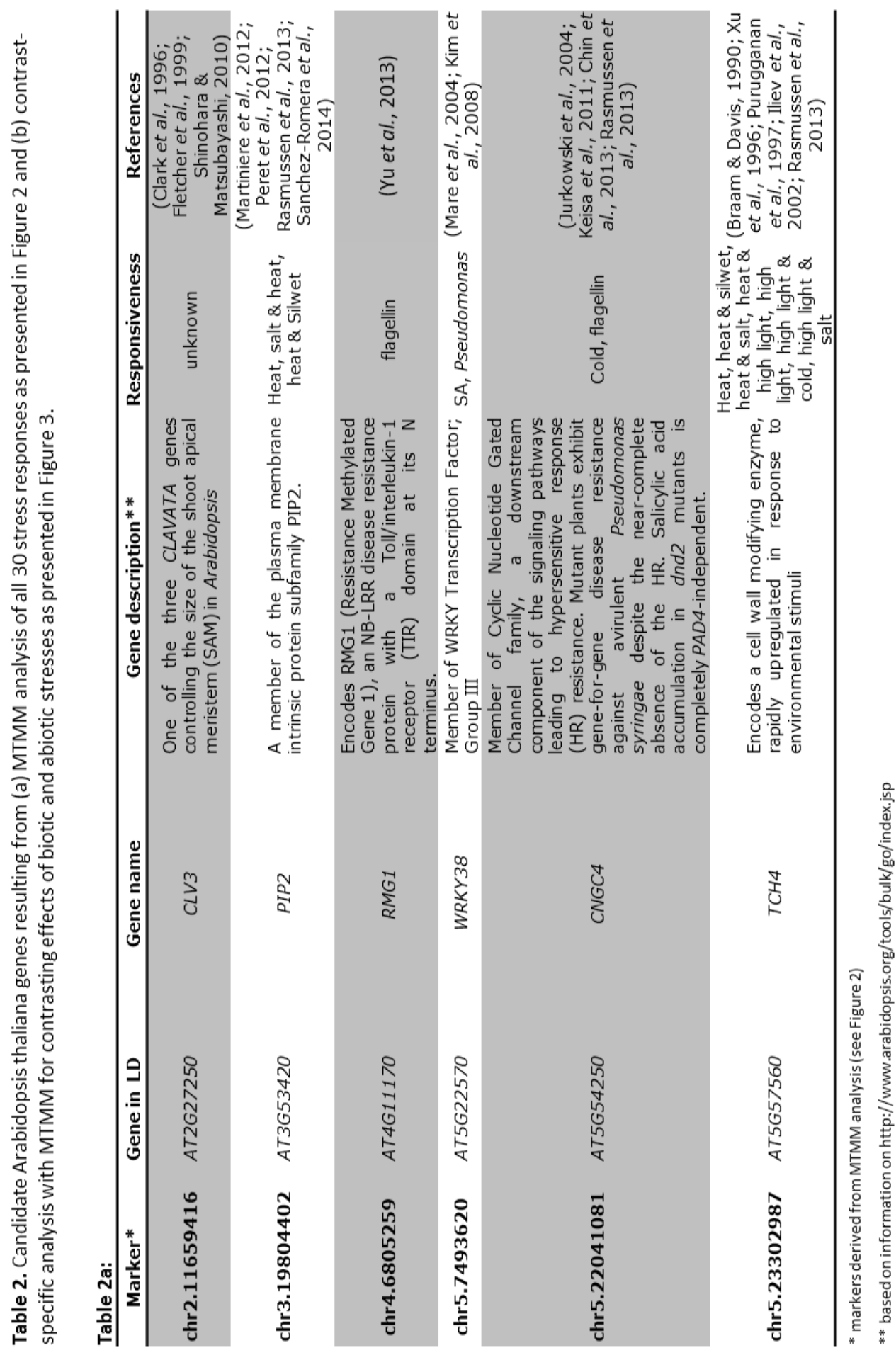




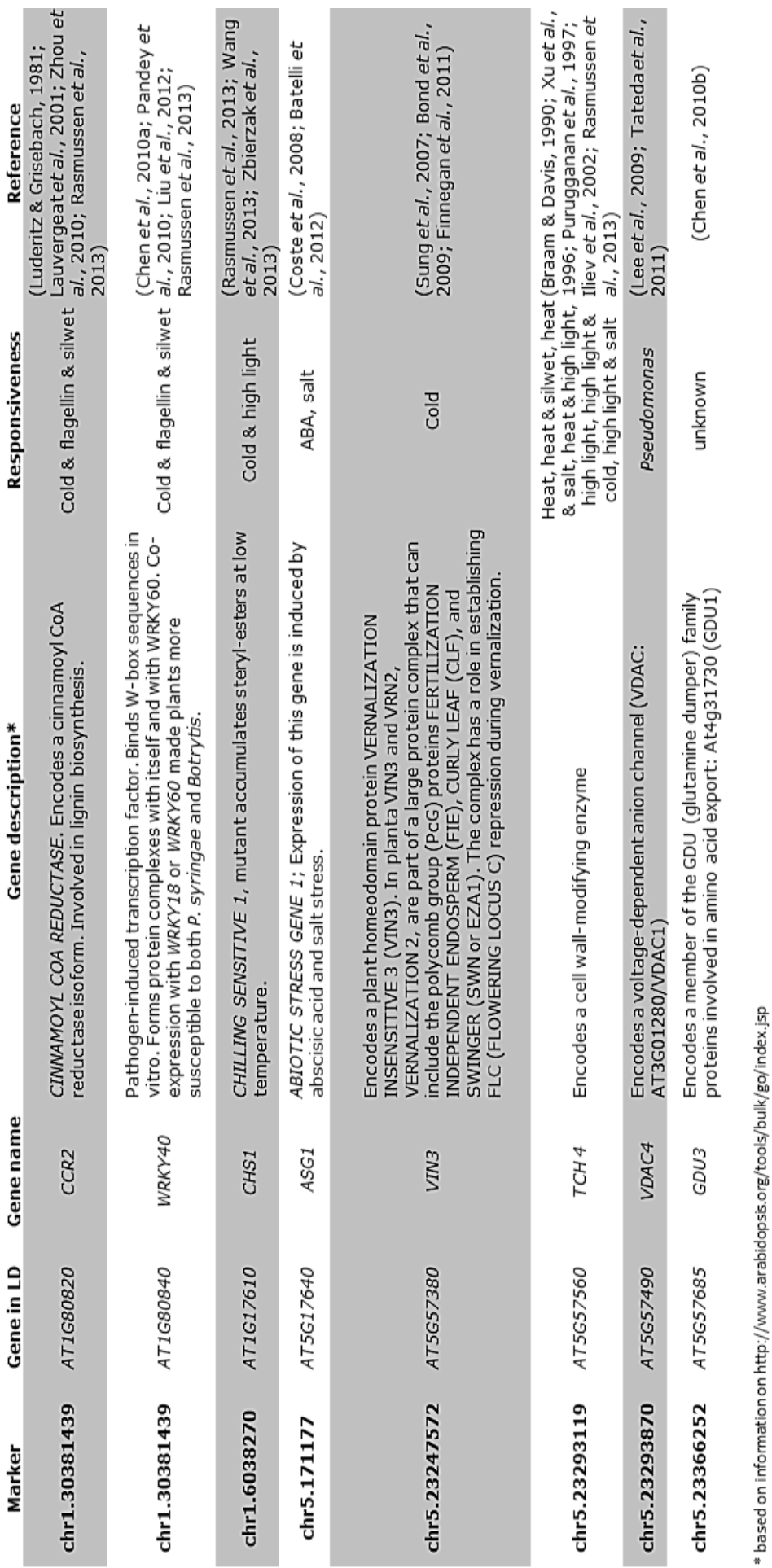




\section{Phytohormonal signaling underlying contrasts in stress}

\section{responses}

The MTMM framework allowed imposing constraints on the values of the estimated QTL effects, thereby providing a powerful testing framework for QTLS that have a common effect for the stresses belonging to one particular group of stresses as contrasted to the effect for another group of stresses (see Materials and Methods section 'Multi-trait GWAS'). We investigated whether polymorphisms for genes involved in SA and JA biosynthesis or genes responsive to signals from these pathways were the cause of the negative genetic correlations between the groups of traits sharing one or the other phytohormonal signaling pathway. To this end, we performed a multi-trait GWA mapping to test the contrast between: (1) parasitic plant and aphid response, versus (2) the most negatively correlated traits, i.e. fungus, caterpillar, thrips and drought response (Figure 1). Fifteen SNPs were significantly associated with contrasting effects between the two trait clusters (Figure S4). Seven of these SNPs, were in LD with one or more genes known to be involved in JA-, SA- or resistance-related signal transduction (Table S4). Among these genes are LOX5, whose product is involved in facilitating aphid feeding (Nalam et al., 2012a; Nalam et al., 2012b), MYB107 encoding a transcription factor responsive to SA (Stracke et al., 2001; Chen et al., 2006), the JA-inducible genes TPSO2 and TPSO3 encoding terpene synthases (Huang et al., 2010) and MES16, encoding a methyl jasmonate esterase (Christ et al., 2012). Using TAIR10 annotations, we found that in total there are 371 genes that have an annotation related to JA and SA signaling (JA-SA genes). Our GWA analysis identified significant SNPS inside or in a $20 \mathrm{~kb}$ neighbourhood of five of those. In the remainder of the genome, i.e. non JA-SA, we identified 162 genes close to or with significant SNPS. So, in candidate regions for JA-SA, we had a ratio of $5 / 371=1.35 \%$ significant genes, while in non-candidate regions, we found $162 / 27863=0.58 \%$. This is an enrichment of 2.33 times, significant at $a=0.05$ (Fisher exact probability test, mid-P value < 0.046; Rivals et al. (2007)). Following Atwell et al. (2010), an upper bound for the false discovery rate is then $1 / 2.33=0.43$.

In addition to screening for SNPs with contrasting effects, we screened for SNPs with a similar effect across the above-mentioned trait clusters (Figure S5) 
and found candidate genes involved in oxidative stress and plant responses to salinity and pathogens (Table S5).

\section{QTLs underlying contrasts in responses to biotic and abiotic stresses}

We expected a negative correlation between the responses to abiotic and biotic stresses due to antagonistic interactions between $A B A$ and the SA and JA/ET pathways (Anderson et al., 2004; Fujita et al., 2006; De Torres Zabala et al., 2009; Kissoudis et al., 2015). Testing for this contrast within the GWA analysis using our MTMM approach significantly identified 43 SNPs with a QTL effect that changed sign between biotic and abiotic conditions. For presentation purposes, traits were grouped by a cluster analysis across SNPs, while SNPs were grouped by clustering across traits. Figure 3 shows the SNPs with the strongest overall effects, identified in 18 LD intervals. The minor alleles of nine of these SNPs displayed a positive effect on biotic stress response traits and a negative effect on abiotic response traits. The remaining nine SNPs displayed the opposite effect (Figure 3). Several candidate genes were identified in LD with the SNPs that are specific for plant responses to either abiotic or biotic stresses (Table 2b), such as TCH4 (encoding a cell-wall modifying enzyme), AtCCR2 (involvement in lignin biosynthesis) and ASG1 (a gene induced by ABA and salt stress). Transcription data (Figure S6) support the notion that these genes play a contrasting role in responses to abiotic and biotic stresses and reveal an antagonistic responsiveness between $\mathrm{ABA}$ and $\mathrm{JA}$ treatment ( $T \mathrm{CH} 4$ ) or a specific responsiveness to either ABA (AtCCR2, ASG1, ATVDAC4) or JA (ATWRKY40). This is in line with the hypothesis that there are antagonistic effects between abiotic stress responses, predominantly involving the ABA pathway, and wound and biotic stress responses involving the JA-ET or SA pathways (Kissoudis et al., 2015). Previous studies have, however, also revealed an overlap in abiotic and biotic plant responses, such as similar transcriptomic perturbations after salinity and pathogens stress (Ma et al., 2006). A screen for QTLs with similar effects on resistance to biotic and abiotic stress (Figure S7) identified three genes annotated to be responsive to stress stimuli (Table S6). Transcriptional data show that these genes respond differentially to different (a)biotic stresses and phytohormones (Figure S8). ARGAH2, encoding an arginase enzyme with a role 
in the metabolism of polyamines and nitric oxide, is involved in both SA- and JAmediated resistance to both biotrophic and necrotrophic pathogens, and is also responsive to abiotic stimuli such as temperature, salt and light intensity (Figure S8) (Jubault et al., 2008; Gravot et al., 2012; Rasmussen et al., 2013). PKS1 is known to be involved in adaptation in plant growth in response to light (Fankhauser et al., 1999; Molas \& Kiss, 2008), but also seems to be responsive to Botrytis (Figure S8). These genes are promising candidates for consistent effects across biotic and abiotic stresses. 
e

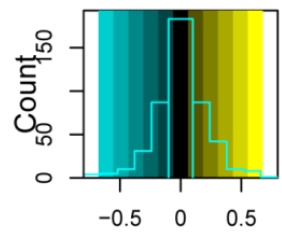

Abiotic- Biotic (specific)

Effect size

Plant response to:

$\square$ abiotic

$\square$ biotic stresses
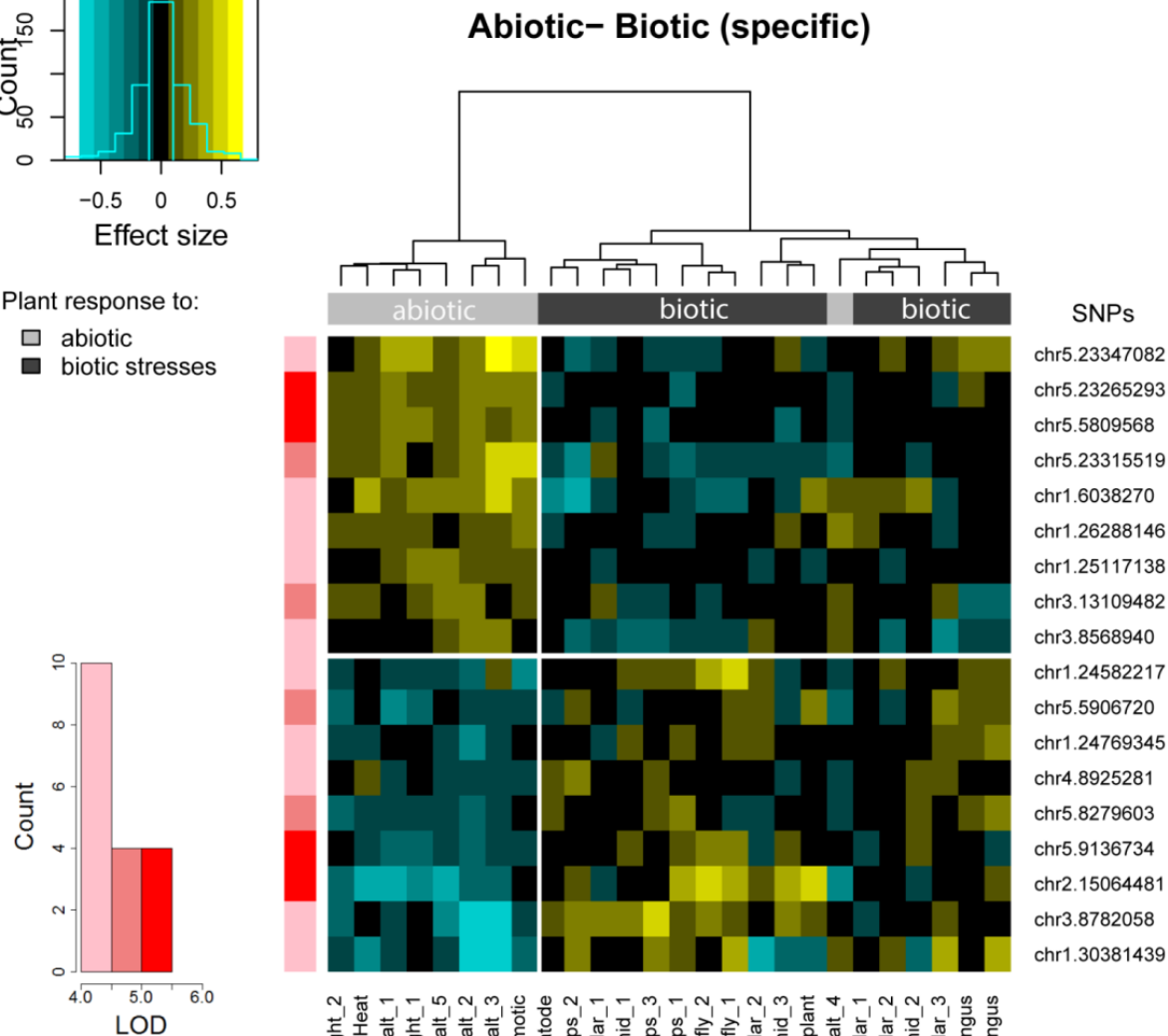

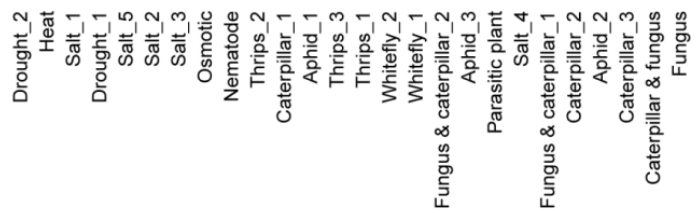

Figure 3. Genetic associations specific for contrasting responses of Arabidopsis thaliana to abiotic and biotic stresses. Genetic associations (in red) were estimated with a contrast-specific GWA analysis using MTMM. For exploratory purposes, significant SNPs $\left(P \leq 10^{-4}\right)$ for the biotic-abiotic contrast were clustered on their trait-specific effect sizes as estimated in the full MTMM, that is, without imposing a contrast restriction on the SNP effects. If there was another SNP in LD that had a higher effect size, this SNP was used a representative for the LD block. Negative effects (blue) were cases where the rare allele was associated with a detrimental effect on the plants, positive effects (yellow) were cases where the rare allele was associated with increased resistance to the stress. The rare alleles of the top 9 SNPs are associated with enhanced resistance to abiotic stresses and reduced resistance to biotic stresses; the bottom 9 SNPs show the inverse. Stresses were clustered on the basis of SNP effects using Ward's minimum variance method. The key shows the frequency distribution of SNPs across effect sizes. 


\section{QTLs underlying contrasts in responses to below- and aboveground stresses}

We expected a negative correlation between responses to below- and aboveground stresses. A strong QTL signal was found on chromosome 1 for this contrasting response (Figure S9). The associated marker (chr1. 13729757) had 12 genes in LD with it, of which 11 are annotated as pseudogenes. Transcriptional data on abiotic stresses for the only protein coding gene (AT1G36510) shows an upregulation in aboveground tissues, yet a downregulation in the root tissues (Winter et al., 2007). Marker chr5.16012837 showed the strongest signal for similar effects on responses to below- and aboveground stresses (Figure S10) for which the pathogenesis-related thaumatin superfamily protein (AT5G40020) is the most promising candidate gene.

\section{Validation of identified QTLS}

To obtain experimental support for the most interesting QTLs resulting from the MTMM, we tested homozygous T-DNA insertion lines for candidate genes RMG1 and WRKY38 (both resulting from the MTMM analysis), and TCH4 (from MTMM analysis on biotic versus abiotic contrast) for several of the stresses addressed in this study. Two independent rmg1 T-DNA insertion lines showed a phenotype that was different from the wild type (Col-0) for some of the stress conditions (Figure 4, Supplementary Methods Section SM.11), being more resistant to caterpillar feeding and osmotic stress (Figure 4). RMG1 (AT4G11170) encodes an NB-LRR disease resistance protein, which acts as a patternrecognition receptor (PRR) that recognizes evolutionarily conserved pathogenderived signatures, and transcription is induced by the bacterial peptide flg22 (Yu et al., 2013). The rare allele of the corresponding marker chr4.6805259 is associated with enhanced resistance to salt stress and the combined stresses 'caterpillar and drought' and 'caterpillar and fungus' and with enhanced susceptibility to drought stress. Gene expression data show that RMG1 is upregulated by several abiotic and biotic stresses (Figure 4). In addition, gene ontology enrichment analysis of the co-expression network of RMG1 shows an overrepresentation of genes involved in immune responses and maintenance of ion homeostasis. The latter is based upon co-expression with five genes 
encoding glutamate receptors (GLR1.2, GLR1.3, GLR2.5, GLR2.8, and GLR2.9), putatively involved in ion-influx-mediated long-distance signaling of wound, pathogen and salt stress (Ma et al., 2006; Mousavi et al., 2013; Choi et al., 2014; Kissoudis et al., 2015). T-DNA insertion lines for TCH4 and WRKY38 did not show a phenotype different from the wild type (Col-0) for any of the tested stress conditions. Whether this is dependent on the genetic background used, remains to be investigated. 

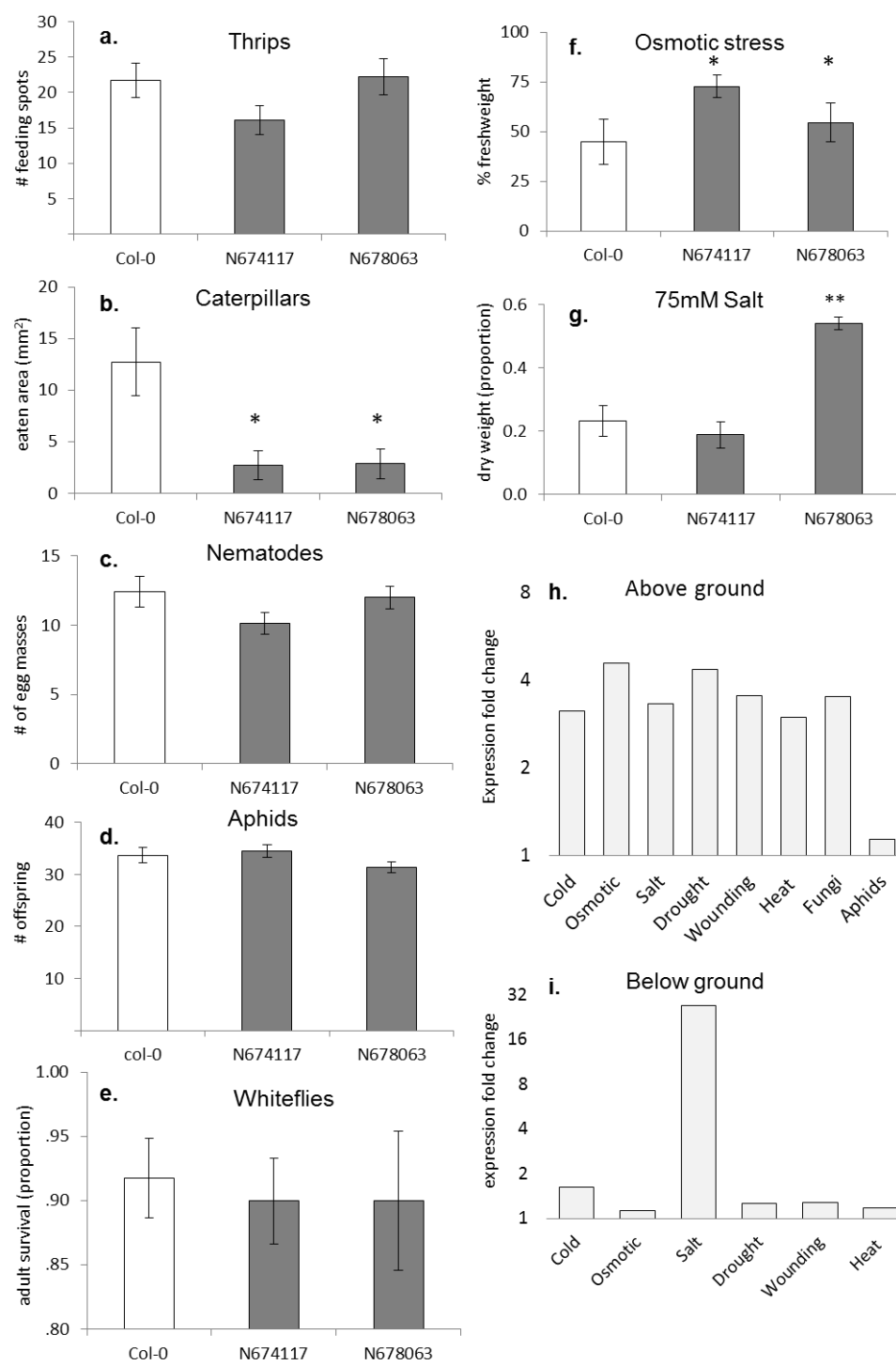

8 h. Above ground
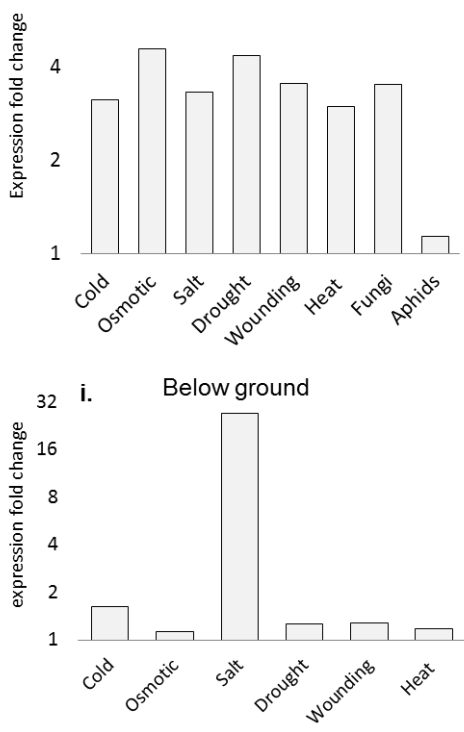

Figure 4. Phenotypes of RMG1 T-DNA mutant screenings for Arabidopsis thaliana. Phenotypes are given for two T-DNA lines in the RMG1 gene and for Col-0 as control. a. Number of thrips feeding spots on a detached leaf, 6 days post infestation $(N=24)$; b. Leaf area consumed by $P$. rapae caterpillars $(\mathrm{N}=6)$; c. Number of nematode egg masses $(\mathrm{N}=23)$; d. Number of $M$. persicae aphid offspring $(\mathrm{N}=10-17)$; e. Percent survival of adult whiteflies $(A$. proletella) $(\mathrm{N}=10)$; f. Plant fresh weight after osmotic treatment in comparison to control (\% relative to control) $(\mathrm{N}=4)$; g. Plant dry weight after $75 \mathrm{mM}$ salt treatment in comparison to control (ratio) $(\mathrm{N}=7-10)$; Mean $\pm \mathrm{SE},+: \mathrm{P}<0.10, *$ : $\mathrm{P}<0.05$, $* *: \mathrm{P}<0.01$, difference in comparison to Col-0. Relative expression fold change for RMG1 compared to untreated control plants in aboveground (h) and belowground (i) tissue. Expression data from Arabidopsis eFP browser (http://bbc.botany.utoronto.ca). 
Summarizing, our multi-trait GWA methodology facilitated a detailed analysis of the genetic architecture of resistance in Arabidopsis to a wide diversity of biotic and abiotic stresses. Application of this methodology revealed novel candidate genes associated with multiple stress responses, where specific contrasts were identified with some genes positively associated with the resistance to one set of stresses while being negatively associated with another set of stresses. In plant breeding (Brady et al., 2005; Ballesteros et al., 2015), such genes are classified as adaptive. Alternatively, other genes were identified with consistent effects across a wide spectrum of stress conditions. Such genes are labelled as constitutive in the plant breeding literature (Brady et al., 2005; Ballesteros et al., 2015). Both adaptive and constitutive QTLs are important factors to contribute to improved stress resistance and tolerance in commercial crop species (Brady et al., 2005; Ballesteros et al., 2015). 


\section{Discussion}

We developed a novel mixed-model approach to multi-trait GWA mapping with a special feature for testing contrasts between groups of stresses to identify the genetic architecture underlying a total of 30 stress response traits in Arabidopsis. The strength of our statistical approach was that our multi-trait mixed model accounted simultaneously for dependencies between genotypes and between traits, providing a natural and appropriate correction for multiple testing, while maximizing power for the detection of QTLs for the stress contrast under study. As we addressed a large number of stresses, our phenotyping experiments were distributed across a series of laboratories and were not performed simultaneously. To mitigate as much as possible the occurrence of QTLs induced purely by experiment-specific differences in plant management and environmental control, our phenotypic responses were defined in terms of control-corrected responses. This type of correction will emphasize QTLs for resistance and tolerance per se and will decrease detection power for QTLS related to development and viability.

The extensive phenotyping executed in this study was done under carefully controlled conditions in climate chambers. Ideally, phenotyping should be done in nature because that is where genetic variation is exposed to natural selection (Bergelson \& Roux, 2010; Brachi et al., 2010; Brachi et al., 2013). Here, we have phenotyped the plant population to 15 different stresses under laboratory conditions and our data show an interesting pattern based on genetic correlations that matches with phytohormonal signalling underlying stress responses (Figure 1). This indicates that the genetic architecture recorded here is biologically relevant. Drought and salt stress responses share signaltransduction mechanisms (Zhu, 2002) which is represented by the genetic correlations recorded (Figure 1 ). Insect damage is commonly associated with drought or osmotic stress and this is also clear from overlap in underlying phytohormonal signalling (Pieterse et al., 2012). Figure 1 shows that drought stress and osmotic stress correlate with insect stresses. Extending studies of genetic variation and the genetic architecture underlying responses to multiple stresses to natural conditions will be an important next step (Bergelson \& Roux, 2010). 
Through the approach developed here, candidate genes for stress responses were identified that are involved in contrasting responses when comparing biotic and abiotic stresses, above- and belowground stresses, and attack by phloem feeders compared with other biotic stresses. Among these genes many are involved in phytohormone-mediated processes, supporting the notion that the phytohormonal regulatory network plays an important role in plant stress responses (Pieterse et al., 2012). The MTMM approach further showed that certain SNPs were associated to multiple stress responses and that transcriptional patterns of genes to which the SNPs were linked, as well as the phenotype expressed upon knocking out one of these genes, matched with the observed stress responses of the plants. The RMG1 gene that was identified through this procedure has relevant effects on plant phenotype in the context of responses to individual stresses. RMG1 is a bacterium-inducible resistance gene whose activity is modulated by the plant through RNA-directed DNA methylation (RdDM) (Yu et al., 2013). RMG1 expression activates the SA pathway (Yu et al., 2013). Thus, the increased resistance against caterpillars in rmg1 mutants may be the result of elimination of SA-mediated interference with JA-induced resistance to caterpillars (Pieterse et al., 2012). RMG1 appears to be inducible by several stresses and deserves further in-depth analysis for its role in plant response to multiple stresses. Our data show that for the 30 most significant SNPs resulting from the MTMM analysis, the average absolute effect size for double stresses is on average higher than that for single stresses $(P<0.007$, Table S2). This suggests that resistance mechanisms involved in countering dual stresses are of a more general nature, in contrast to the rather specific resistance mechanisms involved in single stress responses. However, the combined stresses included in this study especially involve fungal and caterpillar stresses. Future studies including other combined stresses are needed to further investigate the suggested pattern.

The MTMM framework that we used for GWA mapping provides unbiased estimates for QTL allele substitution effects together with correct standard errors for these effects. Within the same framework we developed unique facilities to test hypotheses on QTL-by-stress interactions in multi-trait models, which are not available in competing meta-analysis approaches (Zhu et al., 2015). The variance-covariance structure that we used for the polygenic term protects 
against inflated type I error, i.e. too many false positive SNP-trait associations, as a consequence of population structure and kinship on the genotypic side and genetic correlations between traits on the trait side. The inclusion of trait correlations will, for most QTLs, improve the power of detection in comparison to single-trait GWA mapping (Korte et al., 2012; Zhou \& Stephens, 2014; section 'Multi-trait GWAS' in Materials and Methods). For a comparison of the MTMM analysis with single-trait analyses, see 'Simulations' in Materials and Methods, SM. 12 and Figures S11 and S12. Our choice for the variance-covariance structure of the polygenic term as a Kronecker product of a compressed kinship on the genotypes with an approximated unstructured variance-covariance model on the environments is sometimes used in plant breeding for genomic prediction models (Burgueno et al., 2012). However, implementation of such models in GWA mapping and especially on the scale that we present here, with 30 traits, is unprecedented and is practically far from straightforward. It required substantial work on preparatory phenotypic analyses as well as fine-tuning of the genotypic and trait variance-covariance structures to achieve convergence of the mixed models.

The MTMM analyses identified candidate genes associated with contrasting responses to biotic and abiotic stresses. Stress combinations appeared to have a strong influence on the MTMM outcome, indicative for significant interactions between different stresses when occurring simultaneously, and underlining the importance of studying the resistance of plants to combinations of stress. Transcriptional data and phenotyping of mutants provide initial support for the role of several of the candidate genes identified. Studies of plant responses to a diverse set of biotic stresses show that the transcriptional pattern is stressspecific and that phytohormonal signaling pathways can explain up to $70 \%$ of the induced gene regulation (De Vos et al., 2005). Taking the outcome of the MTMM analyses to investigate the involvement of identified candidate genes in the resistance of plants to several stresses, not only in Arabidopsis but also in related crop species such as e.g. Brassica species will be valuable in the breeding by design of future crops to protect them against combinations of stresses, including biotic and abiotic stresses. This will be of great value for next generation crops. 


\section{Acknowledgements}

This research is supported by the Perspective Programme 'Learning from Nature' of the Dutch Technology Foundation STW, which is part of the Netherlands Organisation for Scientific Research (NWO), and which is partly funded by the Ministry of Economic Affairs.

Author contributions: MPMT, NHDO, KJK, SC, PPH, JAB-M, CB, JBu, JB-L, XC, EFF, MMJ, WL, JAvP, SW, and GLW phenotyped the plants, MPMT, NHDO, KJK, SC, PPH, MGMA, JAB-M, JBa, HJB, JBu, CB, XC, EFF, MAJ, MMJ, JJBK, WL, CMJP, CR-S, GS, CT, JJAvL, JAvP, CCVS, SCMvW, RGFV, RV, BV, DV, SW, GLW, and MD designed the phenotyping experiments and made initial analyses, $\mathrm{WK}, \mathrm{JvH}$ and FAvE developed the multi-trait mixed model, WK, JvH, MPMT, NHDO, KJK, SC, $\mathrm{PPH}, \mathrm{BU}, \mathrm{FAvE}$ and MD analyzed the total dataset. MD and FAvE coordinated the study as a whole. MPMT, NHDO, KJK, SC, PPH, WK, JVH, FAvE and MD wrote the manuscript with input from JJBK, CJMP and MGMA and all authors proofread the final version of the manuscript. 


\section{References}

Alimi NA, Bink MCAM, Dieleman JA, Magán JJ, Wubs AM, Palloix A, Van Eeuwijk FA. 2013. Multi-trait and multi-environment QTL analyses of yield and a set of physiological traits in pepper. Theoretical and Applied Genetics 126: 2597-2625.

Alonso-Blanco C, Aarts MGM, Bentsink L, Keurentjes JJB, Reymond M, Vreugdenhil D, Koornneef M. 2009. What has natural variation taught us about plant development, physiology, and adaptation? Plant Cell 21: 1877-1896.

Anderson JP, Badruzsaufari E, Schenk PM, Manners JM, Desmond OJ, Ehlert C, Maclean DJ, Ebert PR, Kazan K. 2004. Antagonistic interaction between abscisic acid and jasmonate-ethylene signaling pathways modulates defense gene expression and disease resistance in Arabidopsis. Plant Cell 16: 3460-3479.

Atwell S, Huang YS, Vilhjalmsson BJ, Willems G, Horton M, Li Y, Meng D, Platt A, Tarone AM, Hu TT, et al. 2010. Genome-wide association study of 107 phenotypes in Arabidopsis thaliana inbred lines. Nature 465: 627-631.

Bac-Molenaar JA, Fradin EF, Becker FFM, Rienstra JA, van der Schoot J, Vreugdenhil D, Keurentjes JJB. 2015. Genome-wide association mapping of fertility reduction upon heat stress reveals developmental stage-specific QTLs in Arabidopsis thaliana. Plant Cell 27: 1857-1874

Baldacci-Cresp F, Maucourt M, Deborde C, Pierre O, Moing A, Brouquisse R, Favery B, Frendo P. 2015. Maturation of nematode-induced galls in Medicago truncatula is related to water status and primary metabolism modifications. Plant Science 232: 77-85.

Ballesteros DC, Mason RE, Addison CK, Acuna MA, Arguello MN, Subramanian N, Miller RG, Sater H, Gbur EE, Miller D, et al. 2015. Tolerance of wheat to vegetative stage soil waterlogging is conditioned by both constitutive and adaptive QTL. Euphytica 201: 329-343.

Batelli G, Massarelli I, Van Oosten M, Nurcato R, Vannini C, Raimondi G, Leone A, Zhu JK, Maggio A, Grillo S. 2012. Asg1 is a stress-inducible gene which increases stomatal resistance in salt stressed potato. Journal of Plant Physiology 169: 1849-1857.

Baxter I, Brazelton JN, Yu DN, Huang YS, Lahner B, Yakubova E, Li Y, Bergelson J, Borevitz JO, Nordborg M, et al. 2010. A coastal cline in sodium accumulation in Arabidopsis thaliana is driven by natural variation of the sodium transporter AtHKT1;1. Plos Genetics 6: e1001193.

Benjamini, Y, Hochberg, Y. 1995. Controlling the false discovery rate: a practical and powerful approach to multiple testing. Journal of the Royal Statistical Society. Series B (Methodological) 57: 289-300.

Bergelson J, Roux F. 2010. Towards identifying genes underlying ecologically relevant traits in Arabidopsis thaliana. Nature Reviews Genetics 11: 867-879.

Boer MP, Wright D, Feng L, Podlich DW, Luo L, Cooper M, Van Eeuwijk FA. 2007. A mixed-model quantitative trait loci (QTL) analysis for multiple-environment trial data using environmental covariables for QTL-by- environment interactions, with an example in maize. Genetics 177: 1801-1813.

Bond DM, Wilson IW, Dennis ES, Pogson BJ, Jean Finnegan E. 2009. VERNALIZATION INSENSITIVE 3 (VIN3) is required for the response of Arabidopsis thaliana seedlings exposed to low oxygen conditions. Plant Journal 59: 576-587.

Braam J, Davis RW. 1990. Rain-induced, wind-induced, and touch-induced expression of calmodulin and calmodulin-related genes in Arabidopsis. Cell 60: 357-364.

Brachi B, Faure N, Bergelson J, Cuguen J, Roux F. 2013. Genome-wide association mapping of flowering time in Arabidopsis thaliana in nature: genetics for underlying components and reaction norms across two successive years. Acta Botanica Gallica 160: 205-218.

Brachi B, Faure N, Horton M, Flahauw E, Vazquez A, Nordborg M, Bergelson J, Cuguen J, Roux F. 2010. Linkage and association mapping of Arabidopsis thaliana flowering time in nature. Plos Genetics 6: 17. 
Brachi B, Meyer CG, Villoutreix R, Platt A, Morton TC, Roux F, Bergelson J. 2015. Coselected genes determine adaptive variation in herbivore resistance throughout the native range of Arabidopsis thaliana. Proceedings of the National Academy of Sciences of the United States of America 112: 4032-4037.

Bradbury PJ, Zhang Z, Kroon DE, Casstevens TM, Ramdoss Y, Buckler ES. 2007. Tassel: software for association mapping of complex traits in diverse samples. Bioinformatics 23: 2633-2635.

Brady KU, Kruckeberg AR, Bradshaw HD. 2005. Evolutionary ecology of plant adaptation to serpentine soils. Annual Review of Ecology Evolution and Systematics 36: 243-266.

Braun H-J, Rajaram S, van Ginkel M. 1996. CIMMYT's approach to breeding for wide adaptation. Euphytica 92: 175-183.

Burgueno J, de los Campos G, Weigel K, Crossa J. 2012. Genomic prediction of breeding values when modeling Genotype $x$ Environment interaction using pedigree and dense molecular markers. Crop Science 52: 707-719.

Butler DG, Cullis BR, Gilmour AR, Gogel BJ. 2009. ASReml-R reference manual. Queensland Department of Primary Industries and Fisheries, Brisbane, Australia.

Chao DY, Silva A, Baxter I, Huang YS, Nordborg M, Danku J, Lahner B, Yakubova E, Salt DE. 2012. Genome-wide association studies identify heavy metal ATPase 3 as the primary determinant of natural variation in leaf cadmium in Arabidopsis thaliana. Plos Genetics 8: e1002923.

Chen H, Lai Z, Shi J, Xiao Y, Chen Z, Xu X. 2010a. Roles of Arabidopsis WRKY18, WRKY40 and WRKY60 transcription factors in plant responses to abscisic acid and abiotic stress. BMC Plant Biol 10: 281.

Chen $H$, Zhang $Z$, Teng $K$, Lai J, Zhang $Y$, Huang $Y$, Li $Y$, Liang L, Wang $Y$, Chu C, et al. 2010b. Up-regulation of LSB1/GDU3 affects geminivirus infection by activating the salicylic acid pathway. Plant Journal 62: 12-23.

Chen YH, Yang XY, He K, Liu MH, Li JG, Gao ZF, Lin ZQ, Zhang YF, Wang XX, Qiu XM, et al. 2006. The MYB transcription factor superfamily of arabidopsis: Expression analysis and phylogenetic comparison with the rice MYB family. Plant Molecular Biology 60: 107-124.

Chin K, DeFalco TA, Moeder W, Yoshioka K. 2013. The Arabidopsis cyclic nucleotide-gated ion channels AtCNGC2 and AtCNGC4 work in the same signaling pathway to regulate pathogen defense and floral transition. Plant Physiology 163: 611-624.

Choi WG, Toyota M, Kim SH, Hilleary R, Gilroy S. 2014. Salt stress-induced $\mathrm{Ca} 2+$ waves are associated with rapid, long-distance root-to-shoot signaling in plants. Proceedings of the National Academy of Sciences of the United States of America 111: 6497-6502.

Christ B, Schelbert S, Aubry S, Sussenbacher I, Muller T, Krautler B, Hortensteiner S. 2012. MES16, a member of the methyl esterase protein family, specifically demethylates fluorescent chlorophyll catabolites during chlorophyll breakdown in Arabidopsis Plant Physiology 158: 628-641.

Clark SE, Jacobsen SE, Levin JZ, Meyerowitz EM. 1996. The CLAVATA and SHOOT MERISTEMLESS loci competitively regulate meristem activity in Arabidopsis. Development 122: 1567-1575. $636 \mathrm{pp}$.

Cooper M, Hammer GL. 1996. Plant adaptation and crop improvement. IRRI.

Cooper M, Messina CD, Podlich D, Totir LR, Baumgarten A, Hausmann NJ, Wright D, Graham G. 2014. Predicting the future of plant breeding: complementing empirical evaluation with genetic prediction. Crop \& Pasture Science 65: 311-336.

Coste AT, Ramsdale M, Ischer F, Sanglard D. 2008. Divergent functions of three Candida albicans zinc-cluster transcription factors (CTA4, ASG1 and CTF1) complementing pleiotropic drug resistance in Saccharomyces cerevisiae. Microbiology 154: 1491-1501.

De los Campos G, Hickey JM, Pong-Wong R, Daetwyler HD, Calus MPL. 2013. Whole-genome regression and prediction methods applied to plant and animal breeding. Genetics 193: 327-345. 
De Torres Zabala M, Bennett MH, Truman WH, Grant MR. 2009. Antagonism between salicylic and abscisic acid reflects early host-pathogen conflict and moulds plant defence responses. Plant Journal 59: 375-386.

De Vos M, Van Oosten VR, Van Poecke RMP, Van Pelt JA, Pozo MJ, Mueller MJ, Buchala AJ, Metraux JP, Van Loon LC, Dicke M, et al. 2005. Signal signature and transcriptome changes of Arabidopsis during pathogen and insect attack. Molecular PlantMicrobe Interactions 18: 923-937.

De Vos M, Van Zaanen W, Koornneef A, Korzelius JP, Dicke M, Van Loon LC, Pieterse CMJ. 2006. Herbivore-induced resistance against microbial pathogens in Arabidopsis. Plant Physiology 142: 352-363.

Devlin B, Roeder K. 1999. Genomic control for association studies. Biometrics 55: $997-1004$.

Devlin B, Roeder K, Wasserman L. 2001. Genomic control, a new approach to genetic-based association studies. Theoretical Population Biology 60: 155-166.

Dorr I, Kollmann R. 1995. Symplasmic sieve element continuity between Orobanche and its host. Botanica Acta 108: 47-55.

El-Soda M, Kruijer W, Malosetti M, Koornneef M, Aarts MGM. 2015. Quantitative trait loci and candidate genes underlying genotype by environment interaction in the response of Arabidopsis thaliana to drought. Plant Cell and Environment 38: 585599.

Fankhauser C, Yeh KC, Lagarias JC, Zhang H, Elich TD, Chory J. 1999. PKS1, a substrate phosphorylated by phytochrome that modulates light signaling in Arabidopsis. Science 284: 1539-1541.

Finnegan EJ, Bond DM, Buzas DM, Goodrich J, Helliwell CA, Tamada Y, Yun JY, Amasino RM, Dennis ES. 2011. Polycomb proteins regulate the quantitative induction of VERNALIZATION INSENSITIVE 3 in response to low temperatures. Plant Journal 65: 382-391.

Fiorani F, Schurr U 2013. Future Scenarios for Plant Phenotyping. Annual Review of Plant Biology 64: 267-291.

Fletcher JC, Brand U, Running MP, Simon R, Meyerowitz EM. 1999.

Signaling of cell fate decisions by CLAVATA3 in Arabidopsis shoot meristems. Science $\mathbf{2 8 3}$ : 1911-1914.

Fujita M, Fujita Y, Noutoshi Y, Takahashi F, Narusaka Y, YamaguchiShinozaki K, Shinozaki K. 2006. Crosstalk between abiotic and biotic stress responses: a current view from the points of convergence in the stress signaling networks. Current Opinion in Plant Biology 9: 436-442.

Granier C, Vile D. 2014. Phenotyping and beyond: modelling the relationships between traits. Current Opinion in Plant Biology 18: 96-102.

Gravot A, Deleu C, Wagner G, Lariagon C, Lugan R, Todd C, Wendehenne D, Delourme R, Bouchereau A, Manzanares-Dauleux MJ. 2012. Arginase Induction Represses Gall Development During Clubroot Infection in Arabidopsis. Plant and Cell Physiology 53: 901-911.

Holopainen JK, Gershenzon J. 2010. Multiple stress factors and the emission of plant VOCs. Trends in Plant Science 15: 176-184.

Horton MW, Hancock AM, Huang YS, Toomajian C, Atwell S, Auton A, Muliyati NW, Platt A, Sperone FG, Vilhjalmsson BJ, et al. 2012. Genome-wide patterns of genetic variation in worldwide Arabidopsis thaliana accessions from the RegMap panel. Nature Genetics 44: 212-216.

Howe GA, Jander G. 2008. Plant immunity to insect herbivores. Annual Review of Plant Biology 59: 41-66.

Huang MS, Abel C, Sohrabi R, Petri J, Haupt I, Cosimano J, Gershenzon J, Tholl D. 2010. Variation of herbivore-induced volatile terpenes among Arabidopsis ecotypes depends on allelic differences and subcellular targeting of two terpene synthases, TPS02 and TPS03. Plant Physiology 153: 1293-1310.

Iliev EA, Xu W, Polisensky DH, Oh MH, Torisky RS, Clouse SD, Braam J. 2002. Transcriptional and posttranscriptional regulation of Arabidopsis $\mathrm{TCH} 4$ expression 
by diverse stimuli. Roles of cis regions and brassinosteroids. Plant Physiology 130: 770783.

Jubault M, Hamon C, Gravot A, Lariagon C, Delourme R, Bouchereau A, Manzanares-Dauleux MJ. 2008. Differential regulation of root arginine catabolism and polyamine metabolism in clubroot-susceptible and partially resistant Arabidopsis genotypes. Plant Physiology 146: 2008-2019.

Julkowska MM, Testerink C. 2015. Tuning plant signaling and growth to survive salt. Trends in Plant Science 20: 586-594.

Jurkowski GI, Smith RK, Jr., Yu IC, Ham JH, Sharma SB, Klessig DF, Fengler KA, Bent AF. 2004. Arabidopsis DND2, a second cyclic nucleotide-gated ion channel gene for which mutation causes the "defense, no death" phenotype. Molecular Plant-Microbe Interactions 17: 511-520.

Keisa A, Kanberga-Silina K, Nakurte I, Kunga L, Rostoks N. 2011.

Differential disease resistance response in the barley necrotic mutant nec1. BMC Plant Biology 11: 66.

Kerwin R, Feusier J, Corwin J, Rubin M, Lin C, Muok A, Larson B, Li BH, Joseph B, Francisco M, et al. 2015. Natural genetic variation in Arabidopsis thaliana defense metabolism genes modulates field fitness. eLife 4: e05604.

Kim KC, Lai ZB, Fan BF, Chen ZX. 2008. Arabidopsis WRKY38 and WRKY62 transcription factors interact with histone deacetylase 19 in basal defense. Plant Cell 20: 2357-2371.

Kim S, Plagnol V, Hu TT, Toomajian C, Clark RM, Ossowski S, Ecker JR, Weigel D, Nordborg M. 2007. Recombination and linkage disequilibrium in Arabidopsis thaliana. Nature Genetics 39: 1151-1155.

Kissoudis C, Chowdhury R, van Heusden S, van de Wiel C, Finkers R,

Visser RF, Bai Y, van der Linden G. 2015. Combined biotic and abiotic stress resistance in tomato. Euphytica 202: 317-332.

Kissoudis C, van de Wiel C, Visser RGF, van der Linden G. 2014. Enhancing crop resilience to combined abiotic and biotic stress through the dissection of physiological and molecular crosstalk. Frontiers in Plant Science 5: 207.

Kloth KJ, ten Broeke CJM, Thoen MPM, den Brink MHV, Wiegers GL, Krips

OE, Noldus L, Dicke M, Jongsma MA. 2015. High-throughput phenotyping of plant resistance to aphids by automated video tracking. Plant Methods 11: 4.

Kooke R, Kruijer W, Bours R, Becker F, Kuhn A, van de Geest H, Buntjer J, Doeswijk T, Guerra J, Bouwmeester $H$, et al. 2016. Genome-wide association mapping and genomic prediction elucidate the genetic architecture of morphological traits in Arabidopsis. Plant Physiology 170: 2187-2203.

Korte A, Vilhjalmsson BJ, Segura V, Platt A, Long Q, Nordborg M. 2012. A mixed-model approach for genome-wide association studies of correlated traits in structured populations. Nature Genetics 44: 1066-1071.

Kruijer W, Boer MP, Malosetti M, Flood PJ, Engel B, Kooke R, Keurentjes JJB, van Eeuwijk FA. 2015. Marker-based estimation of heritability in immortal populations. Genetics 199: 379-393.

Lauvergeat V, Lacomme C, Lacombe E, Lasserre E, Roby D, GrimaPettenati J. 2001. Two cinnamoyl-CoA reductase (CCR) genes from Arabidopsis thaliana are differentially expressed during development and in response to infection with pathogenic bacteria. Phytochemistry 57: 1187-1195.

Lee SM, Hoang MH, Han HJ, Kim HS, Lee K, Kim KE, Kim DH, Lee SY, Chung WS. 2009. Pathogen inducible voltage-dependent anion channel (AtVDAC) isoforms are localized to mitochondria membrane in Arabidopsis. Molecules and Cells 27: 321-327.

Li Y, Huang Y, Bergelson J, Nordborg M, Borevitz JO. 2010. Association mapping of local climate-sensitive quantitative trait loci in Arabidopsis thaliana. Proceedings of the National Academy of Sciences of the United States of America 107: 21199-21204.

Liu ZQ, Yan L, Wu Z, Mei C, Lu K, Yu YT, Liang S, Zhang XF, Wang XF, Zhang DP. 2012. Cooperation of three WRKY-domain transcription factors WRKY18, 
WRKY40, and WRKY60 in repressing two ABA-responsive genes ABI4 and ABI5 in Arabidopsis. Journal of Experimental Botany 63: 6371-6392.

Luderitz T, Grisebach H. 1981. Enzymic synthesis of lignin precursors. Comparison of cinnamoyl-CoA reductase and cinnamyl alcohol:NADP+ dehydrogenase from spruce (Picea abies L.) and soybean (Glycine max L.). European Journal of Biochemistry 119: 115-124.

Ma SS, Gong QQ, Bohnert HJ. 2006. Dissecting salt stress pathways. Journal of Experimental Botany 57: 1097-1107.

Makumburage GB, Richbourg HL, LaTorre KD, Capps A, Chen CX, Stapleton AE. 2013. Genotype to phenotype maps: multiple input abiotic signals combine to produce growth effects via attenuating signaling interactions in maize. G3Genes Genomes Genetics 3: 2195-2204.

Malosetti M, Ribaut JM, van Eeuwijk FA. 2013. The statistical analysis of multi-environment data: modeling genotype-by-environment interaction and its genetic basis. Frontiers in Physiology 4: 17.

Malosetti M, Ribaut JM, Vargas M, Crossa J, Van Eeuwijk FA. 2008. A multitrait multi-environment QTL mixed model with an application to drought and nitrogen stress trials in maize (Zea mays L.). Euphytica 161: 241-257.

Mare C, Mazzucotelli E, Crosatti C, Francia E, Stanca AM, Cattivelli L. 2004. Hv-WRKY38: a new transcription factor involved in cold- and drought-response in barley. Plant Molecular Biology 55: 399-416.

Martiniere A, Li X, Runions J, Lin J, Maurel C, Luu DT. 2012. Salt stress triggers enhanced cycling of Arabidopsis root plasma-membrane aquaporins. Plant Signaling \& Behavior 7: 529-532.

Meijer K. 2009. Factor-analytic models for genotype $\times$ environment type problems and structured covariance matrices. Genetics Selection Evolution 41: 21.

Mittler R, Blumwald E. 2010. Genetic engineering for modern agriculture: Challenges and perspectives. Annual Review of Plant Biology 61: 443-462.

Molas ML, Kiss JZ. 2008. PKS1 plays a role in red-light-based positive phototropism in roots. Plant Cell and Environment 31: 842-849.

Mousavi SAR, Chauvin A, Pascaud F, Kellenberger S, Farmer EE. 2013. GLUTAMATE RECEPTOR-LIKE genes mediate leaf-to-leaf wound signalling. Nature 500: 422-426.

Nalam VJ, Keereetaweep J, Shah J. 2012a. The green peach aphid, Myzus persicae, acquires a LIPOXYGENASE5-derived oxylipin from Arabidopsis thaliana, which promotes colonization of the host plant. Plant Signaling \& Behavior 8: e22735.

Nalam VJ, Keeretaweep J, Sarowar S, Shah J. 2012b. Root-derived oxylipins promote green peach aphid performance on arabidopsis foliage. Plant Cell 24: 1643-1653.

Odong TL, van Heerwaarden J, van Hintum TJL, van Eeuwijk FA, Jansen J. 2013. Improving hierarchical clustering of genotypic data via Principal Component Analysis. Crop Science 53: 1546-1554.

Ogura T, Busch W. 2015. From phenotypes to causal sequences: using genome wide association studies to dissect the sequence basis for variation of plant development. Current Opinion in Plant Biology 23: 98-108.

Pandey SP, Roccaro M, Schon M, Logemann E, Somssich IE. 2010.

Transcriptional reprogramming regulated by WRKY18 and WRKY40 facilitates powdery mildew infection of Arabidopsis. Plant Journal 64: 912-923.

Peret B, Li G, Zhao J, Band LR, Voss U, Postaire O, Luu DT, Da Ines O, Casimiro I, Lucas $\mathbf{M}$, et al. 2012. Auxin regulates aquaporin function to facilitate lateral root emergence. Nature Cell Biology 14: 991-998.

Piepho HP. 1997. Analyzing genotype-environment data by mixed models with multiplicative effects. Biometrics 53: 761-766.

Pierik R, Testerink C. 2014. The art of being flexible: how to escape from shade, salt, and drought. Plant Physiology 166: 5-22.

Pieterse CMJ, Leon-Reyes A, Van der Ent S, Van Wees SCM. 2009. Networking by small-molecule hormones in plant immunity. Nature Chemical Biology $\mathbf{5}$ : 308-316. 
Pieterse CMJ, Van der Does D, Zamioudis C, Leon-Reyes A, Van Wees SCM. 2012. Hormonal modulation of plant immunity. Annual Review of Cell and Developmental Biology 28: 489-521.

Platt A, Horton M, Huang YS, Li Y, Anastasio AE, Mulyati NW, Agren J, Bossdorf O, Byers D, Donohue K, et al. 2010. The scale of population structure in Arabidopsis thaliana. Plos Genetics 6: e1000843.

Prasch CM, Sonnewald U. 2013. Simultaneous application of heat, drought, and virus to Arabidopsis plants reveals significant shifts in signaling networks. Plant Physiology 162: 1849-1866.

Purugganan MM, Braam J, Fry SC. 1997. The Arabidopsis TCH4 xyloglucan endotransglycosylase - Substrate specificity, $\mathrm{pH}$ optimum, and cold tolerance. Plant Physiology 115: 181-190.

Rasmussen S, Barah P, Suarez-Rodriguez MC, Bressendorff S, Friis $\mathbf{P}$, Costantino P, Bones AM, Nielsen HB, Mundy J. 2013. Transcriptome responses to combinations of stresses in Arabidopsis. Plant Physiology 161: 1783-1794.

Rivals I, Personnaz L, Taing L, Potier MC. 2007. Enrichment or depletion of a GO category within a class of genes: which test? Bioinformatics 23: 401-407.

Rivero RM, Mestre TC, Mittler R, Rubio F, Garcia-Sanchez F, Martinez V. 2014. The combined effect of salinity and heat reveals a specific physiological, biochemical and molecular response in tomato plants. Plant Cell and Environment 37: 1059-1073.

Rizhsky L, Liang H, Shuman J, Shulaev V, Davletova S, Mittler R. 2004. When defense pathways collide. The response of Arabidopsis to a combination of drought and heat stress. Plant Physiology 134: 1683-1696.

Runyon JB, Mescher MC, De Moraes CM. 2008. Parasitism by Cuscuta pentagona attenuates host plant defenses against insect herbivores. Plant Physiology 146: 987-995.

Sanchez-Romera B, Ruiz-Lozano JM, Li G, Luu DT, Martinez-Ballesta Mdel C, Carvajal M, Zamarreno AM, Garcia-Mina JM, Maurel C, Aroca R. 2014.

Enhancement of root hydraulic conductivity by methyl jasmonate and the role of calcium and abscisic acid in this process. Plant Cell and Environment 37: 995-1008.

Sewelam N, Oshima Y, Mitsuda N, Ohme-Takagi M. 2014. A step towards understanding plant responses to multiple environmental stresses: a genome-wide study. Plant Cell and Environment 37: 2024-2035.

Shinohara H, Matsubayashi Y. 2010. Arabinosylated glycopeptide hormones: new insights into CLAVATA3 structure. Current Opinion in Plant Biology 13: 515-519.

Stam JM, Kroes A, Li YH, Gols R, van Loon JJA, Poelman EH, Dicke M.

2014. Plant interactions with multiple insect herbivores: from community to genes. Annual Review of Plant Biology 65: 689-713.

Stracke R, Werber M, Weisshaar B. 2001. The R2R3-MYB gene family in Arabidopsis thaliana. Current Opinion in Plant Biology 4: 447-456.

Sung S, Schmitz RJ, Amasino R. 2007. The role of VIN3-LIKE genes in environmentally induced epigenetic regulation of flowering. Plant Signaling and Behavior 2: 127-128.

Suzuki N, Rivero RM, Shulaev V, Blumwald E, Mittler R. 2014. Abiotic and biotic stress combinations. New Phytologist 203: 32-43.

Tateda C, Watanabe K, Kusano T, Takahashi Y. 2011. Molecular and genetic characterization of the gene family encoding the voltage-dependent anion channel in Arabidopsis. Journal of Experimental Botany 62: 4773-4785.

Tjallingii WF, Hogen Esch T. 1993. Fine structure of aphid stylet routes in plant tissues in correlation with EPG signals. Physiological Entomology 18: 317-328.

van Eeuwijk FA, Bink MC, Chenu K, Chapman SC. 2010. Detection and use of QTL for complex traits in multiple environments. Current Opinion in Plant Biology 13: 193205.

van Heerwaarden J, Hufford MB, Ross-Ibarra J. 2012. Historical genomics of North American maize. Proceedings of the National Academy of Sciences of the United States of America 109: 12420-12425. 
van Heerwaarden J, Odong TL, van Eeuwijk FA. 2013. Maximizing genetic differentiation in core collections by PCA-based clustering of molecular marker data. Theoretical and Applied Genetics 126: 763-772.

van Rooijen R, Aarts MGM, Harbinson J. 2015. Natural genetic variation for acclimation of photosynthetic light use efficiency to growth irradiance in Arabidopsis. Plant Physiology 167: 1412-1429.

Vile D, Pervent M, Belluau M, Vasseur F, Bresson J, Muller B, Granier C, Simonneau T. 2012. Arabidopsis growth under prolonged high temperature and water deficit: independent or interactive effects? Plant Cell and Environment 35: 702-718.

Wang $Y$, Zhang $Y$, Wang $Z$, Zhang $X$, Yang S. 2013. A missense mutation in CHS1, a TIR-NB protein, induces chilling sensitivity in Arabidopsis. Plant Journal 75: 553565.

Wijnen CL, Keurentjes JJB. 2014. Genetic resources for quantitative trait analysis: novelty and efficiency in design from an Arabidopsis perspective. Current Opinion in Plant Biology 18: 103-109.

Winter D, Vinegar B, Nahal H, Ammar R, Wilson GV, Provart NJ. 2007. An "Electronic Fluorescent Pictograph" Browser for Exploring and Analyzing Large-Scale Biological Data Sets. PLOS ONE 2: 12.

Xu W, Campbell P, Vargheese AK, Braam J. 1996. The Arabidopsis XETrelated gene family: Environmental and hormonal regulation of expression. Plant Journal 9: 879-889.

Yu A, Lepere G, Jay F, Wang JY, Bapaume L, Wang Y, Abraham AL, Penterman J, Fischer RL, Voinnet $\mathbf{O}$, et al. 2013. Dynamics and biological relevance of DNA demethylation in Arabidopsis antibacterial defense. Proceedings of the National Academy of Sciences of the United States of America 110: 2389-2394.

Zbierzak AM, Porfirova S, Griebel T, Melzer M, Parker JE, Dormann P. 2013. A TIR-NBS protein encoded by Arabidopsis Chilling Sensitive 1 (CHS1) limits chloroplast damage and cell death at low temperature. Plant Journal 75: 539-552.

Zhang Z, Ersoz E, Lai CQ, Todhunter RT, Tiwari HK, Gore MA, Bradbury PJ, Yu J, Arnett DK, Ordovas JM, et al. 2010. Mixed linear model approach adapted for genome-wide association studies. Nature Genetics 42: 355-360.

Zhou R, Jackson L, Shadle G, Nakashima J, Temple S, Chen F, Dixon RA. 2010. Distinct cinnamoyl CoA reductases involved in parallel routes to lignin in Medicago truncatula. Proceedings of the National Academy of Sciences of the United States of America 107: 17803-17808.

Zhou X, Stephens M. 2014. Efficient multivariate linear mixed model algorithms for genome-wide association studies. Nature Methods 11: 407-409.

Zhu JK. 2002. Salt and drought stress signal transduction in plants. Annual Revies of Plant Biology 53: 247-273.

Zhu XF, Feng T, Tayo BO, Liang JJ, Young JH, Franceschini N, Smith JA, Yanek LR, Sun YV, Edwards TL, et al. 2015. Meta-analysis of correlated traits via summary statistics from GWASs with an application in hypertension. American Journal of Human Genetics 96: 21-36. 


\section{Supporting Information}

Additional supporting information may be download via link

https://www.dropbox.com/sh/hb95t5dwvxbsc1u/AAB2Z7Q1DPSwCCUoaTBBi2Pa?dl=0

\section{Supplementary tables}

Table S1: Data overview on phenotyping the 350 Arabidopsis thaliana accessions of the HapMap collection. trait.

Table S2: Summed effect sizes of 30 most significant SNPs in MTMM per

Table S3: 125 candidate genes derived from the Multitrait Mixed Model analysis.

Table S4: Genes in linkage with SNPs with - $\log _{10}(P)$ score above 4 (20 kb half-window size) in the contrast-specific GWA mapping of parasitic plants and aphids on the one hand versus fungus, caterpillar, thrips and drought on the other hand.

Table S5: Candidate genes in linkage with SNPs with $-\log _{10}(P)$ score above 4 (20 kb half-window size) that have common effects on plant response to parasitic plants and aphids on the one hand versus fungus, caterpillar, thrips and drought on the other hand.

Table S6: Candidate genes in linkage with SNPs with $-\log _{10}(P)$ score above 4 (20 kb half-window size) that have common effects on biotic and abiotic stress responses. 


\section{Supplementary figures}

Figure S1: Narrow sense heritability for Arabidopsis thaliana resistance to abiotic and biotic stresses.

Figure S2: Genetic and phenotypic correlation matrix.

Figure S3: Expression data of 6 candidate genes (resulting from MTMM, see Table 2a) in plants exposed to biotic or abiotic stress factors, relative to control conditions.

Figure S4: Genetic associations specific for plant responses to the main clusters of the genetic correlation network (see Figure 1): parasitic plant and aphid versus fungus, caterpillar, thrips and drought.

Figure S5: Genetic associations common for plant response to the main clusters of the genetic correlation network: parasitic plant and aphid on the one hand versus fungus, caterpillar, thrips and drought on the other hand.

Figure S6: Expression data of 6 candidate genes (resulting from MTMM analysis, see Table $2 \mathrm{~b}$ ) in plants exposed to biotic or abiotic stress factors, relative to control conditions.

Figure S7: Genetic associations common for plant responses to abiotic and biotic stresses.

Figure S8: Expression data of 3 candidate genes (resulting from MTMM, see Supplementary Table S6) in plants exposed to biotic or abiotic stress factors, relative to control conditions.

Figure 59: Genetic associations specific for plant responses to either below- or aboveground stress.

Figure S10: Genetic associations common for plant responses to below- and aboveground stresses.

Figure S11: Power of MTMM in simulations.

Figure S12: Comparison of SNPS identified by MTMM and Univariate GWAS. 


\section{Supplementary methods}

SM.1 Salt

SM.2 Abiotic

SM.3 Caterpillar - combinatory stress

SM.4 Parasitic plants

SM.5 Nematodes

SM.6 Whiteflies

SM.7 Aphids

SM.8 Thrips

SM.9 Drought - combinatory stress

SM.10 Fungus - combinatory stress

SM.11 Screening of T-DNA lines 


\section{$\mathbb{C} \| \Delta \mathbb{P} \mathbb{\|} \mathbb{R} \mathbb{R} 3$}

\section{Genome wide association analysis reveals candidate genes controlling a negative effect of biotic stresses on consecutive osmotic stress response}

Pingping Huang ${ }^{1}$, Mohamed El-Soda ${ }^{2}$, Katarzyna W. Wolinska ${ }^{1}$, Nelson H. Davila Olivas $^{4}$, Joop J.A. Van Loon ${ }^{3}$, Marcel Dicke ${ }^{3}$, Kaige Zhao ${ }^{1}$, Jan van Kan ${ }^{4}$, Mark G. M. Aarts ${ }^{1}$

\footnotetext{
${ }^{1}$ Laboratory of Genetics, Wageningen University, Droevendaalsesteeg 1, 6708 PB, Wageningen, the Netherlands.

${ }^{2}$ Department of Genetics, Faculty of Agriculture, Cairo University, Giza, 12613, Egypt.

${ }^{3}$ Laboratory of Entomology, Wageningen University, Wageningen, The Netherlands.

${ }^{4}$ Laboratory of Phytopathology, Wageningen University, Droevendaalsesteeg 1, 6708 PB, Wageningen, the Netherlands.
} 


\section{Abstract}

While the response of Arabidopsis thaliana to either drought, herbivory or fungal infection has been well-examined, the consequences of exposure to a series of such (a)biotic stresses are not well studied. This work reports on the genetic mechanisms underlying the Arabidopsis thaliana (Arabidopsis) response to a priming pre-treatment with a biotic agent, followed by drought, a 'combinatorial stress' treatment. Two genome-wide association (GWA) mapping approaches were employed on a set of 350 Arabidopsis accessions. Plants were exposed separately to either fungal infection using Botrytis cinerea or herbivory using Pieris rapae caterpillars, and then subjected to a PEG treatment to simulate drought. Alternatively, plants were subjected to PEG without any pretreatment. Multi-environment (ME) GWA-mapping identified a number of SNPs associated with candidate genes for which allelic variation affects the plant response to combinatorial stresses in a gene-by-environment interaction (GXE) mode. Univariate GWA-mapping identified additional SNPs that affect phenotypic plasticity in response to the combinatorial stress treatment when compared to the single PEG treatment. SNPs associated with a gene of unknown function and DROUGHT INDUCED 19 (DI19) were identified by both approaches, supporting their potential involvement in the combinatorial stress response. Several SNPs were found to be in linkage disequilibrium with known stress-responsive genes such as PEROXIDASE 34 (PRX34), BASIC LEUCINE ZIPPER 25 (bZIP25), RESISTANCE METHYLATED GENE 1 (RMG1), and WHITE RUST RESISTANCE 4 (WRR4). New roles were found for some of these known stress-responsive genes, e.g. for PRX34 in their response to the combined Pieris and PEG treatment, and RMG1 to Botrytis and PEG. In general, antagonistic interactions were found between biotic and drought stress responses, and found a negative effect of biotic stresses on consecutive osmotic stress response. The results of this study illustrate the complexity of combinatorial stress responses, but also offer new leads to improve tolerance of crop species to multiple stresses. 


\section{Keywords}

Arabidopsis thaliana, genome-wide association mapping, genotype-byenvironment interaction, QTL x E, (a)biotic stress response, multiple stress. 


\section{Introduction}

In the field, plants are simultaneously or sequentially exposed to various biotic and abiotic stress inducing factors which limit plant performance and cause a substantial annual reduction in agricultural production (Hirt and Shinozaki, 2004). Plant responses to biotic and abiotic stresses are mainly regulated by abscisic acid ( $A B A$ ), in response to abiotic stresses such as drought and salt (Shinozaki and Yamaguchi-Shinozaki, 2007); jasmonic acid (JA) and ethylene (ET), in response to necrotrophic fungi and chewing insects; and salicylic acid (SA), which activates the plant defence response upon infection with biotrophic fungi (Pieterse et al., 2009; Dodds and Rathjen, 2010). Alongside these major compounds, plant hormones such as brassinosteroids (BRs), auxins (AUXs), and cytokinins ( $\mathrm{CKs}$ ) are also involved in regulating the response of plants to biotic and abiotic stresses (Hirt, 2009; Pareek et al., 2010; Pieterse et al., 2012). Negative interactions have been observed between JA and $S A, A B A$ and $S A, A B A$ and $J A$, and $A B A$ and $E T$; while positive interactions have been observed between ABA and AUX (Yoshioka and Shinozaki, 2009).

The interaction between biotic and abiotic stress responses is often mediated by different plant hormones and the outcome can be synergistic, antagonistic, or neutral. The impact of abiotic stresses on plant responses biotic strains can significantly, and vice versa (Atkinson and Urwin, 2012; Appel et al., 2014; Rejeb et al., 2014). For instance, drought increased ABA concentrations have a positive impact on callose deposition, which may boost plant resistance to fungal and bacterial pathogens (Mauch-Mani and Mauch, 2005; Achuo et al., 2006; Ramegowda et al., 2013). Turnip Mosaic Virus (TuMV), which causes lesions, mosaics, and mottling that reduce photosynthetic capacity, thereby rendering the plant more susceptible to subsequent drought stress (Prasch and Sonnewald, 2015).

Generally, plant responses to individual stress factors are different from those involved in countering detrimental triggers that are either simultaneous or sequentially combined. The consecutive exposure to biotic and abiotic stresses triggers specific multiple-stress-responsive gene expression, indicating the existence of a unique combinatorial stress signalling network (Atkinson and Urwin, 2012; Sewelam et al., 2014). For instance, Rasmussen et al (2013) 
showed that $61 \%$ transcription changes in response to the combinatorial stress cannot be predicted from the single stress responses. Prasch and Sonnewald (2013) showed that the expression of the resistance gene RPS6 was only regulated in case of simultaneous heat, drought, and virus treatments, but not under any of the double or individual stresses. Transcriptome analysis on simultaneous effects of drought, heat, and virus infections in Arabidopsis resulted in identification of 11 genes involved in all relevant single, double, and triple stress treatments (Prasch and Sonnewald, 2013), with those responding to stress factor combinations impossible to determine in presence of single triggers (Rasmussen et al., 2013).

Most studies on the response of plants to multiple stresses have only focused on a limited number of genotypes (Narsai et al., 2013; Shaik and Ramakrishna, 2013; Sewelam et al., 2014; Ramegowda and Senthil-Kumar, 2015), which is fine to understand the general physiology of the response, but insufficient to understand the genetic architecture of such response. For Arabidopsis it is attractive to investigate the natural genetic variation of a trait using genome-wide association (GWA) mapping, which gives high genetic resolution for traits which have high heritability and are controlled by a modest number of genes (Baxter et al., 2010; Li et al., 2010; Chao et al., 2012; BacMolenaar et al., 2015; Ogura and Busch, 2015).

In the research reported here, the genetic architecture of the Arabidopsis response to a biotic stress, followed by drought, was examined. Arabidopsis plants were either exposed to Pieris rapae larvae, to induce response to herbivory, or to the necrotrophic fungus Botrytis cinerea, to induce a fungal infection response, and then subjected to a PEG treatment which induces an osmotic stress, simulating drought. These two treatments will be further referred to as 'Pieris and PEG' and 'Botrytis and PEG'. Control plants were subjected to the single PEG treatment without any pre-treatment. The purpose of our study was to 1 ) determine the heritability of the single and combined treatments for different growth parameters; 2) identify SNPs associated with these phenotypes through different GWA approaches; 3) identify candidate genes linked to these SNPs, for which allelic variation contributes to the trait variation; and 4) 
determine if there is any genotype by environment interaction affecting the phenotypes, that could be linked to allelic variation. 


\section{Materials and Methods}

\section{Plant material and growth conditions.}

For the GWA-mapping experiments, the Arabidopsis HapMap population ( $\mathrm{Li}$ et al., 2010) was used. Seeds of most T-DNA insertion mutants (Supplementary Table 1) were ordered from the European Arabidopsis Stock Centre (http://Arabidopsis.info/), except for seeds of the drought-induced19 (di19) mutant (Liu et al., 2013), which were obtained from the authors.

Prior to experiments, seeds were stratified at $4^{\circ} \mathrm{C}$ in the dark for 5 days. Thereafter, one seed per accession was sown on one Grodan ${ }^{\circ}$ Rockwool cube of 40 X 40 X $40 \mathrm{~mm}$. Plants were watered 3 times / week (on Monday, Wednesday, and Friday, respecitvely) with a nutrient solution developed for Arabidopsis with $\mathrm{pH}=7$ and $\mathrm{EC}=1.4 \mathrm{mS} / \mathrm{cm}$. The nutrient solution consisted of $1.7 \mathrm{mM} \mathrm{NH}_{4}^{+}$, $4.5 \mathrm{mM} \mathrm{K}^{+}, 0.4 \mathrm{mM} \mathrm{Na}^{+}, 2.3 \mathrm{mM} \mathrm{Ca}^{2+}, 1.5 \mathrm{mM} \mathrm{Mg}^{2+}, 4.4 \mathrm{mM} \mathrm{NO}_{3}{ }^{-}, 0.2 \mathrm{mM} \mathrm{Cl}^{-}$, $3.5 \mathrm{mM} \mathrm{SO}_{4}{ }^{2-}, 0.6 \mathrm{mM} \mathrm{HCO}_{3}{ }^{-}, 1.12 \mathrm{mM} \mathrm{PO}_{4}{ }^{3-}, 0.23 \mathrm{mM} \mathrm{SiO}_{3}{ }^{2-}, 21 \mu \mathrm{M} \mathrm{\textrm {Fe } ^ { 2 + }}$ chelated using $3 \%$ diethylene triaminopentaacetic acid, $3.4 \mu \mathrm{M} \mathrm{Mn}^{2+}, 4.7 \mu \mathrm{M}$ $\mathrm{Zn}^{2+}, 14 \mu \mathrm{M} \mathrm{BO}_{3}{ }^{3-}, 6.9 \mu \mathrm{M} \mathrm{Cu}^{2+}$, and $<0.1 \mu \mathrm{M} \mathrm{MoO}_{4}{ }^{4-}$. Plants were grown in a climate-controlled growth chamber set to short day condition, i.e. $10 \mathrm{~h}$ day/14 $\mathrm{h}$ night, at $21^{\circ} \mathrm{C}$ day $/ 19^{\circ} \mathrm{C}$ night temperatures and $70 \%$ relative humidity. Irradiation was set at $200 \mu \mathrm{mol} \mathrm{m} \mathrm{m}^{-2}$.

\section{Preparation of Pieris rapae caterpillar and Botrytis cinerea spores}

Pieris rapae caterpillars were reared on cabbage plants (Brassica oleracea convar. capitata var. alba) under greenhouse conditions $\left(24^{\circ} \mathrm{C}\right.$, with natural daylight). Butterflies were fed on the nectar of flowering plants such as Lantana camara. When flowers were scarce, additional food solution consisting of $20 \%$ honey and $10 \%$ sucrose was introduced to butterflies. Inbreeding of the existing population was minimized by adding wild butterflies and caterpillars from the Dutch Flevopolder. After starving for 1h, first-instar (L1) larvae were placed on Arabidopsis leaves using a fine paint brush as described by Van Wees et al., (2013). 
Botrytis cinerea strain B05.10 (Staats and Van Kan, 2012) was grown for 2 weeks at room temperature on half-strength potato dextrose agar (PDA; Difco Laboratories) plates containing $100 \mu \mathrm{g} / \mathrm{ml}$ penicillin and $200 \mu \mathrm{g} / \mathrm{ml}$ streptomycin. Spores were collected, filtered through glass wool and then resuspended in half-strength potato dextrose broth (PDB; Difco Laboratories) to a final density of $1 \times 10^{5}$ spores/ml. Three hours after incubation, spores were used for inoculation by applying two 5- $\mu$ l droplets on two Arabidopsis leaves as described by Van Wees et al., (2013).

\section{Single and combinatorial stresses}

The HapMap population : For single osmotic treatment, 19-day-old plants were irrigated with Hyponex nutrient solution that contained $7.7 \% \mathrm{w} / \mathrm{v}$ Polyethylene Glycol (PEG) 8000 for 6 days ("PEG"). For combinatorial stress, 17day-old plants were exposed to one Pieris 1st instar larva or inoculated with two $5-\mu l$ droplets of Botrytis spore suspension for 1 day. The next day, the caterpillars were removed and the plant continued to grow in the control conditions for 1 day, followed by irrigation with Hyponex solution that contained $7.7 \% \mathrm{w} / \mathrm{v}$ Polyethylene Glycol (PEG) 8000 for 6 days ("Pieris and PEG" and "Botrytis and PEG" ). Plants that did not receive any stress treatment were used as a control (Figure 1). 
a.

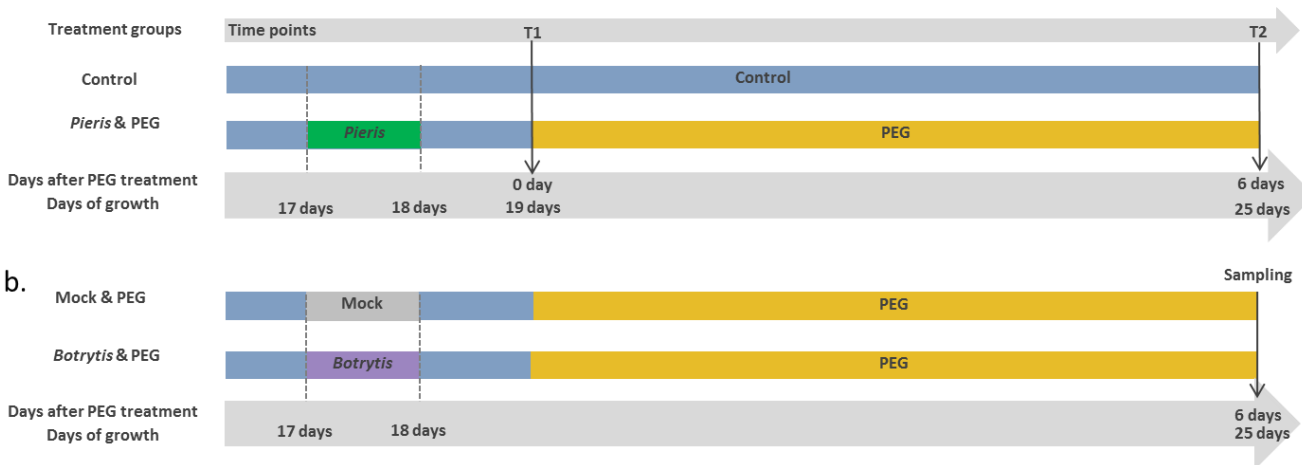

Figure 1. Experimental design for treatment of Arabidopsis plants subjected to Mock and PEG, Botrytis cinerea infection and PEG, or Pieris rapae herbivory and PEG, and control conditions. Conditions are indicated in different colours: control (dark blue); PEG (yellow); Botrytis treatment (purple); Mock treatment (grey); and Pieris treatment (green). Vertical arrows indicate sampling time points ( $\mathrm{T} 1, \mathrm{~T} 2$, Sampling). Vertical dashed line indicates before and after biotic treatment.

Testing T-DNA mutants: In addition to the four treatments described in the previous paragraph, For single Pieris treatment, 17-day-old plants were exposed to either one Pieris $1^{\text {st }}$ instar larva or two $5-\mu l$ droplets of Botrytis spore suspension for 1 day. Thereafter, the caterpillars were removed and the plant was allowed to grow in control conditions for 6 days ("Pieris" and "Botrytis") (Figure 2).

Testing extreme accessions from the HapMap population: This experiment was carried out with the same design as described in the previous paragraph. 


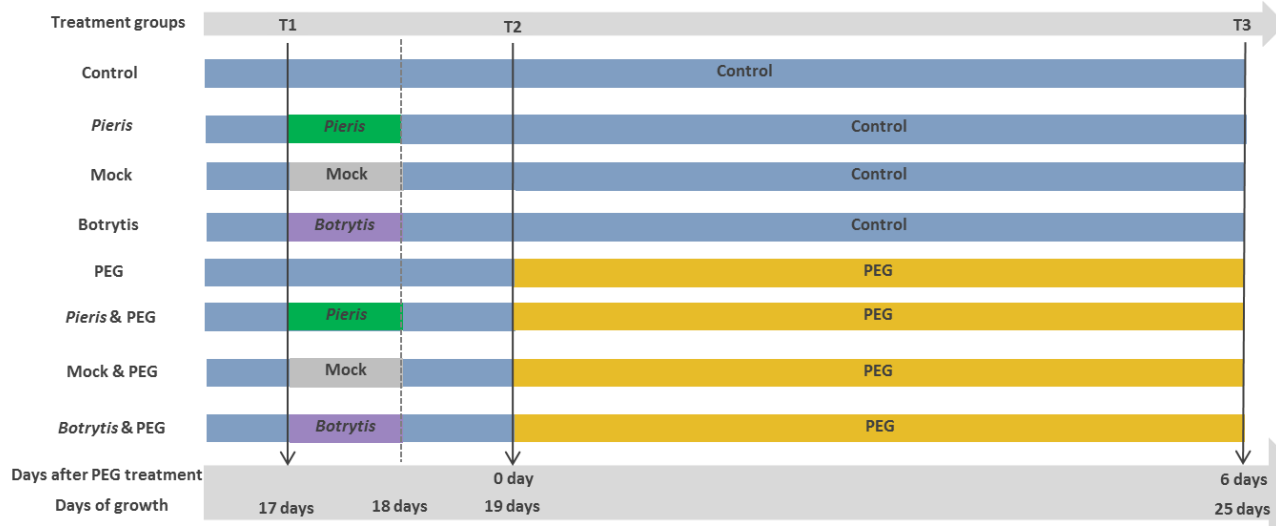

Figure 2. Experimental design for treatment of Arabidopsis plants subjected to PEG, Botrytis cinerea infection, Pieris rapae herbivory, Mock, PEG, Mock and PEG, Botrytis infection and PEG, or Pieris rapae herbivory and PEG, and control conditions. Conditions are indicated in different colours: control (dark blue); PEG (yellow); Botrytis treatment (purple); Mock treatment (grey); and Pieris treatment (green). Vertical arrows indicate sampling time points (T1 - T3). Vertical dashed line indicates after biotic treatment

\section{Plant phenotyping}

For all plants used for GWA mapping, projected Leaf Area (PLA) were measured. For the combined Pieris and PEG treatment, plants were measured directly after the Pieris pre-treatment ( $\mathrm{T} 1)$, and 6 days after the PEG treatment (T2) ( Figure 1.a). For the other treatments the rosettes of too many plants were overlapping and the data was not used for mapping. Instead, for the Botrytis and Mock \& PEG treatments, rosette fresh weight (FW) and dry weight (DW) were measured after the PEG treatment. (Figure 1.b). Rosette water content (WC) was calculated using the formula: $\frac{\text { fresh wight-dryweight }}{\text { fresh weight }}$.

\section{Genome-Wide Association mapping}

For PLA, residuals were calculated from the regression analysis between the PLA after Pieris treatment (T1) and PLA after PEG treatment (T2), whereas, residuals of DW and WC were calculated from the regression between the Botrytis \& PEG and mock \& PEG. These residual values were used for mapping. For Multi-Environment (ME) GWA mapping (Korte et al., 2012) a minor allele frequency (MAF) of 0.05 was used and a $-\log (p$-value) of 4 as arbitrary threshold value for significance as described by El-Soda et al., 2015). Univariate GWA-mapping was performed using the scan_GLS software as described by 
Kang et al. (2010) and Kruijer et al (2015). In brief, this approach involves performing generalized least squares (GLS) calculations conditional on the variance components, which were estimated in the model without markers and can efficiently handle genetically identical individuals. Thereafter, SNPs with MAF $<0.05$ were excluded. The proportion of explained phenotypic variance was the criterion to identify significant SNPs as described by (Sun et al., 2010). The information of SNPs in the linkage disequibrium (LD) with the significant SNPS were identified by the online LD tool (http://dev3.ab.wur.nl/AthaLD), as well as the information of coding sequence substitution and amino acid substitution.

\section{Selection of candidate genes}

Gene ontology annotation of selected candidate genes for the quantitative trait loci (QTL) identified after the GWA analysis was found in The Arabidopsis Information Resource (TAIR; www.Arabidopsis.org/tools/bulk/go/index.jsp). Genes characterized by one or more of the following criteria were considered as candidates: 1) Genes known to be responsive to biotic and abiotic stimulus and expressed under abiotic stress and hormonal (JA and ABA) treatments; 2) Genes with reported physiological functions in response to stress conditions and expressed under abiotic stresses and hormonal (JA and $A B A$ ) treatments (http://bar.utoronto.ca/efp/cgi-bin/efpWeb.cgi). 3) Genes with reported physiological functions in response to stress conditions and responsive to biotic and abiotic stimuli.

T-DNA mutants of the candidate genes were ordered from the European Arabidopsis Stock Centre if available as homozygotes.

\section{Comparing drought and PEG8000-induced osmotic stress}

Four-week-old Col-0 plants were subjected to a PEG treatment as described above and compared to soil-grown plants subjected to drought by withholding water for $6 \mathrm{~d}$. The expression of five drought-responsive genes; $R D 26$ (AT4G27410), MYC2 (AT1G32640), RD29b (AT5G52300), RD29a (AT5G52310), and P5CS1 (AT2G39800) (Shinozaki and Yamaguchi-Shinozaki, 2007) was determined by real-time quantitative reverse transcription PCR ( $q R T-P C R$ ) under both treatments. 
RNA was extracted from rosettes following the protocol described by OnateSanchez and Vicente-Carbajosa (2008). cDNA was synthesized from $800 \mathrm{ng}$ of total RNA using an iScript cDNA synthesis kit (Bio-Rad). cDNA was diluted ten times before qRT-PCR. The gene with ID At3g15930 was selected as reference for qRT-PCR as the most stably expressed across different drought, osmotic, and Botrytis treatments based on the transcriptome database Genevestigator (refgenes.org/rg). The qRT-PCR was performed using iQ SYBR ${ }^{\circledR}$ Green Supermix (cat. no. 170-8885) on a Bio-Rad CFX96 real-time PCR system set at $95^{\circ} \mathrm{C}$ for 4 minutes, followed by 40 cycles of $95^{\circ} \mathrm{C}$ for $10 \mathrm{~s}$ and $55^{\circ} \mathrm{C}$ for $30 \mathrm{~s}$. Relative gene expression was calculated using the $2(-\Delta \Delta C T)$ method (Livak and Schmittgen, 2001). The standard error were calculated from at least 3 plants per accession. Primers of the five drought-responsive genes mentioned in the previous paragraph are provided in Supplementary Table 2. Primers of all investigated candidate genes are provided in Supplementary Table 3.

\section{Statistical analysis}

The broad sense heritability was calculated using the equation $\mathrm{H}^{2}=$ $\frac{\operatorname{Var}(G)}{\operatorname{Var}(G)+\operatorname{Var}(E)}$, in which $\mathrm{G}=$ Genotype, $\mathrm{E}=$ Environment and Var = Variance. Pearson's correlation coefficient ' $r$ ', one-way and two-way ANOVAs were performed using GenStat for Windows $16^{\text {th }}$ Edition (VSN International Ltd., Hemel Hempstead, UK). Student's t-test was used to compare the growth of the same accession between treatments. Three-way ANOVA was performed as described by (Sokal RR, 1981). Statistical tests of FW, DW and WC were performed on 4 plants per accession, whereas gene expression was performed on 3 plants per accession. 


\section{Results}

\section{Drought stress can be mimicked by exposure to polyethylene}

\section{glycol}

To minimize non-genetic, environmental, variation affecting the phenotypes of the Arabidopsis Hapmap population used for GWA analysis, plants were grown on rockwool, watered with nutrient solution, rather than on a peat-based substrate often used in growing Arabidopsis. This however limits the application of drought stress by withholding water, as rockwool is much more resilient to drying than peat-based substrates. To induce a stress mimicking drought, a polyethylene glycol (PEG) 8000 treatment was applied, which gave similar plant phenotypes after six days as plants grown on a peat-sand mixture left without water for six days. To show that also plant physiology was comparable, the expression of five genes involved in the ABA-dependent or the ABA-independent pathway of drought response (Shinozaki and Yamaguchi-Shinozaki, 2007) was determined in plants subjected to either treatment (Figure 3). Except for $R D 29 b$, the change of expression of these genes compared to control conditions, was indistinguishable. $R D 29 \mathrm{~b}$ expression was induced in both treatments, only slightly, but significantly, more in the PEG treatment. Nevertheless, we conclude that the two treatments are sufficiently comparable to consider the PEG treatment as physiologically equivalent to drought by withholding watering, which allowed us to use rock-wool-based growth set-up that supports more uniform growth of Arabidopsis under control and stress treatments than a peatbased growth set-up. 


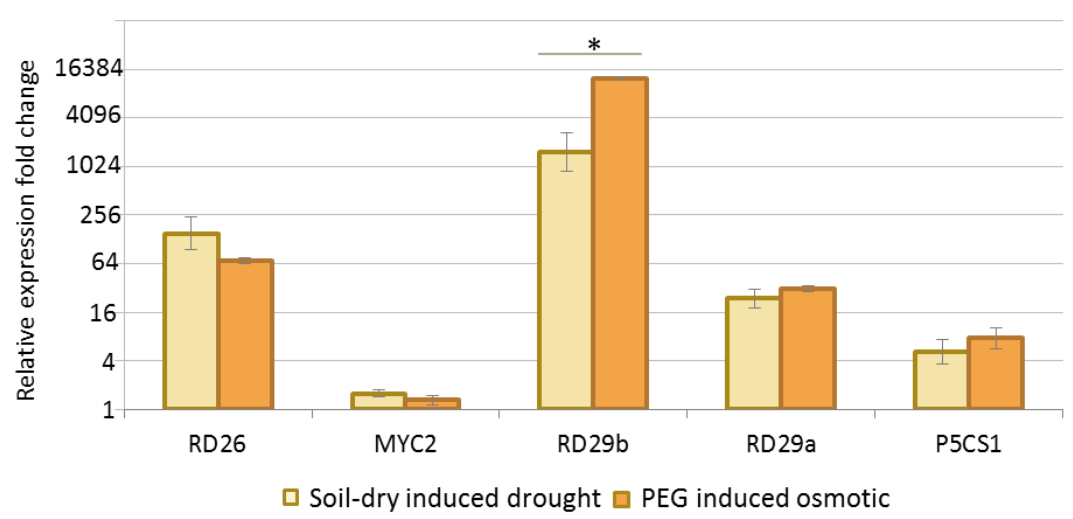

Figure 3. Relative expression of five drought-responsive genes in rosettes of plants either grown on a peat-based mixture, subjected to drought by withholding water (light orange) or grown on rock wool, watered with a nutrient solution containing PEG8000 (PEG), to induce an osmotic stress response (dark orange). Relative gene expression was determined by comparing expression in treated plants with expression in control plants (either growing in a well-watered peat mixture or on rock wool watered with a nutrient solution without PEG). A Student's t-test was used to compare expression of each gene in the different conditions. * indicates a significant difference $(p<0.05)$. Standard errors were calculated based on at least four plants per treatment.

\section{Phenotyping the Hapmap population under the Botrytis and the combinatorial stress}

To obtain relevant trait values for genetic characterization of quantitative trait loci (QTLs) involved in the response to a combined stress treatment, plants of the Hapmap population were grown on rock wool and either pre-treated with Pieris rapae larvae (Pieris treatment), to induce herbivory, or with Botrytis cinerea (Botrytis treatment), to induce fungal infection, before exposure to a PEG treatment to induce osmotic stress (Figure 1-2). In this experiment, rosette fresh weight (FW) and projected rosette leaf area (PLA) were determined in the Pieris treatment and FW, PLA, rosette dry weight (DW) and the (FW-DW)/FW ratio, representing water content (WC) for the Botrytis treatment. The rosette FW measurements of plants exposed to different treatments were all significantly correlated, suggesting no major environmental disturbances or experimental errors (Supplementary Table 4). Broad sense heritabilities $\left(\mathrm{H}^{2}\right)$ were calculated for the measured traits in control and treated plants, which ranged from 0.37 to 0.63 (Supplementary Table 5), suggesting moderate to high heritability and good prospects of identification of QTLs upon mapping. Two-way ANOVA was performed to test the interaction between treatments and 
accessions for PLA, FW, DW, and WC. Significant main effects of treatments and accessions were observed, but no but no significant interactions between accessions and treatments were found (Supplementary Table 6).

\section{Univariate GWA-mapping of residuals identified SNPs associated with phenotypic plasticity}

Rather than mapping each trait for the respective treatment, we decided to map the residual values for each trait and accession combination obtained after regression of the trait value of stress-treated plants on the trait value of controlgrown plants, or of combinatorial stress-treated plants on single stress-treated plants. The residuals represent the variations of the accessions (genotype) in response to stresses (environment) (Filiault and Maloof, 2012). To observe the effect of Pieris priming on plant growth in response to subsequent PEG, residuals was calculated from a regression of PLA of the Pieris and PEG stress treatment on PLA of the Pieris treatment alone. When these PLA residual values were used for GWA analysis, 60 associated SNPs were found, directly linked to 47 genes (Supplementary Figure 2.a, Supplementary Table 7), but with more genes in linkage disequilibrium (LD) with these SNPs. Eleven SNPs were mapped to a genomic region that spans only $19 \mathrm{~kb}$ on chromosome (Chr) 2, in which six genes are located (Figure 4.a), corresponding to TAIR gene IDs At2g36540At $2 \mathrm{~g} 36590$. The combined boxplot of the haplotypes combining nine of these 11 SNPs showed a that accessions containing the non-Col-0 allele had comparably lower rosette PLA under the Pieris and PEG treatment vs. the single Pieris treatment than accessions containing the Col-0 allele (Figure 5.a and Supplementary Figure 2). SNPs m73992 -97 were located at gene with TAIR IDs At2g36560, significantly reduced PLA was observed in when comparing accessions carrying the non-Col allele to the accessions carrying Col-0 allele (Figure 5.b).

When the residuals resulting from the regression of the DW upon Botrytis and PEG treatment on the DW upon PEG treatment alone were used for GWA mapping, 26 associated SNPs were found, residing in 17 genes (Supplementary Figure 2.b, Supplementary Table 8). Nine associated SNPs mapped to a locus spanning $9.7 \mathrm{~kb}$ of Chr 1 , corresponding to four genes with TAIR IDs At1g56280- 
At1g56310 (Figure 4.b). The combined boxplot of the haplotypes combining these nine SNPs (m33786 - m33809) showed a that accessions containing the non-Col-0 allele had comparably higher rosette DW under the Botrytis and PEG treatment vs. the single PEG treatment than accessions containing the Col-0 allele (Figure 5.c). Six (m33789 - m33798) of these 9 SNPs, residing in At1g56290, the non-Col-0 allele of the six SNPs exhibited significant higher DW under the Botrytis and PEG treatment vs. the single PEG treatment than accessions containing the Col-0 allele (Figure 5.d). The SNP marker m33786, residing in the DROUGHT INDUCED 19 (DI19) gene (At1g56280), significantly distinguished accessions carrying the non-Col-0 allele from those carrying the Col-0 allele (Figure 5.e), with the former allele contributing to a relatively higher DW after the combined treatment.

When mapping the residuals for WC, comparing the combined Botrytis and PEG treatment with the single PEG treatment, 23 associated SNPs were found, corresponding to 17 genes (Supplementary Figure 2.c, Supplementary Table 6). A set of SNPs identifies one region of $14.5 \mathrm{~kb}$ on Chr. 5, comprising genes with TAIR IDs At5g06480-At5g06530 (Figure 4.c, Supplementary Table 9). One of these genes is ATP-BINDING CASSETTE TRANSPORTER G22 gene (At5g06530), mutation of which is known to cause increased water respiration and drought susceptibility (Kuromori et al., 2011). Accessions carrying the non-Col-0 haplotype for SNP markers m164494 and particularly m164506, residing in this region, had a lower relative WC upon Botrytis and PEG stress vs. PEG stress alone, when compared to accessions carrying the Col-0 allele (Figure 5.f \& g)

Figure 4. Genomic region on (a) Genomic region on Chromosome 2 from (15324282bp to $1346000 \mathrm{bp}$ ) contains six genes At2g36540-At2g365090 which were associated to 11 significant SNPs for Pieris and PEG responses (Supplementary Table 7). (b) Chromosome 1 from (21072000bp to 21088000bp) contains six genes At1g56280-At1g56330 which were associated to nine significant SNPs for Botrytis and PEG responses(Supplementary Table 8); (b) Chromosome 5 from 1970000bp to 2005000bo) contains five genes At5g06490 At5g06530 which were associated to nine significant SNPs for Botrytis and PEG responses (Supplementary Table 9). Red colour indicates SNPs in high LD (LD > 0.8) with SNP m73995 (a), m33786 (b), or m164484 (c), which exhibited the highest $-\log (p)$ score among the SNPs. Yellow colour indicates SNPs in LD (LD > 0.3) with the SNP m33786, m73995 or m164484. Yellow boxes indicates gene exons. Horizontal dashed line indicates bonferroni threshold. Figure 4 is the GWA-mapping results which was obtained using an accelerated mixed model (AMM) method from online tool GWAPP (https://gwas.gmi.oeaw.ac.at). Same association region was found by both AMM and GLS (this project) methods. 

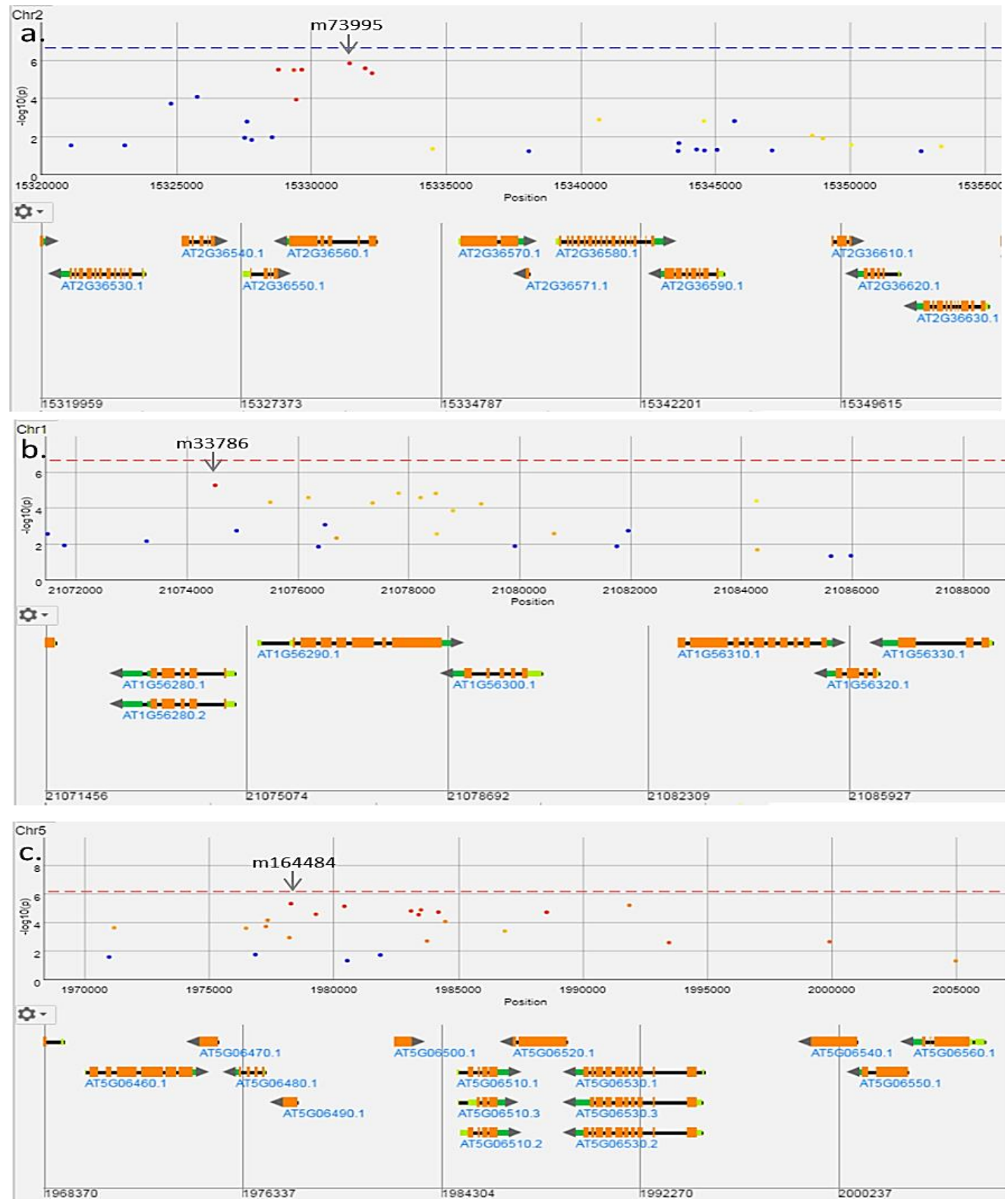

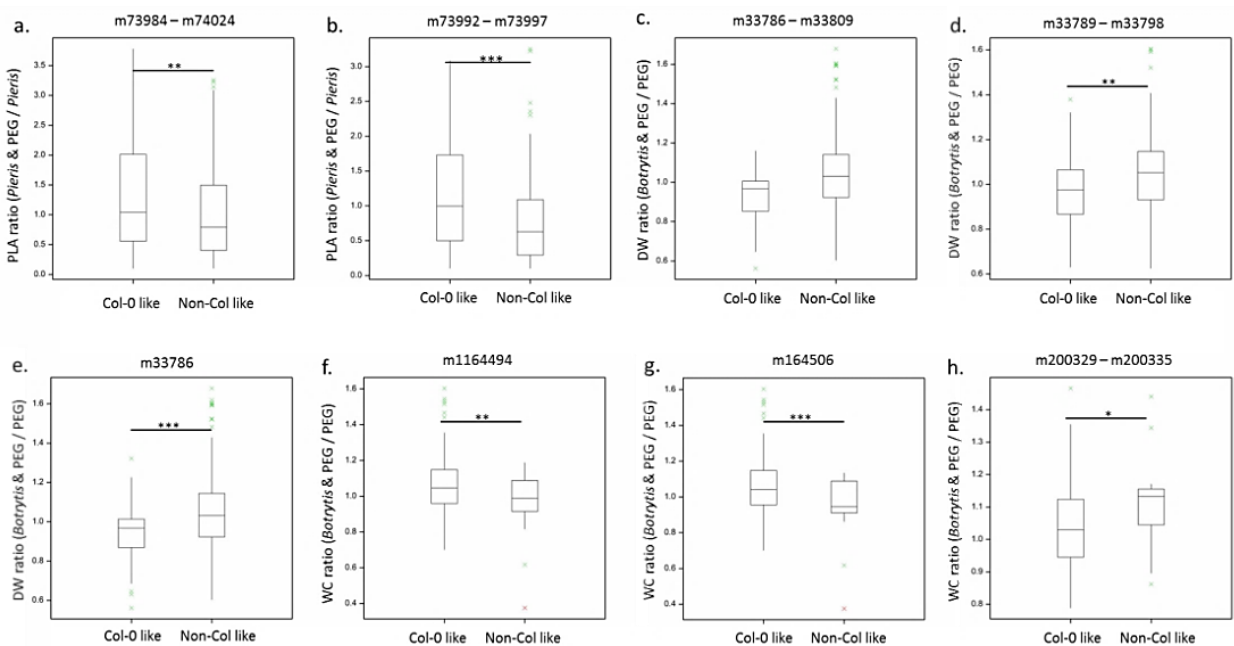

Figure 5. Boxplots shows the effect of two types alleles (Col-0 like and Non-Col like) of the combination of (a) 11 significant SNPs (m73984 - m74024) and (b) six (SNPs m73992 - m73997, residing in At2g36560, on project rosette area (PLA) under the Pieris and PEG treatment vs. the single Pieris treatment; the combination of (c) nine significant SNPs (m33786 - m33809), (d) six significant SNPs (m33789 - m33798, residing in At1g56290), and (e) one significant SNP m33786 (residing in DI19) on rosette dry weight (DW) under the Botrytis and PEG treatment vs. the single PEG treatment; Significant SNPs (e) m1164494, (f) m164506, and m200329 - m200335 on rosette water content (WC) under the Botrytis and PEG treatment vs. the single PEG treatment. Student's t-test was sued to compare the difference of the two groups. *indicates the difference between the Col- 0 like allele and the Non-Col allele is $*<0.05, * *<0.01, * * *<0.001$. 


\section{Multi-environment GWA-mapping identified SNPs interacting with the stress treatments}

Next to the univariate GWA mapping approach, we also employed Multienvironment (ME) GWA-mapping using a multi trait mixed model (MTMM) approach (El-Soda et al., 2015). This approach has the advantage over the first one that it can test multiple environment responses and thus is better in identifying SNPs representing QTL-by-environment interaction (QXE). This method was used to map PLA at the two time-points at which it was determined during the Pieris and PEG treatment, with T1 representing the Pieris pretreatment and T2 representing the Pieris and PEG treatment (Figure 1). This revealed 39 associated SNPs (Supplementary Figure 3.a and supplementary table 9). Seven of these SNPs mapped to a region of Chr 2 containing the genes with TAIR IDs At2g36550 and At2g36560. This region was also found in the univariate GWA analysis (Supplementary Figure 2.a). Accession carrying the non-Col allele of gene with TAIR ID At2g36560 exhibited reduced PLA under the Pieris and PEG treatment vs. the single Pieris treatment when compared to accessions containing the Col-0 allele (Figure 5.b)

When mapping the QXE effect on DW, comparing the combined Botrytis and PEG treatment with the single PEG treatment, 10 associated SNPs, residing in nine genes were found (Supplementary Figure 3.b and supplementary table 10). One of these was the SNP identifying the DI19 gene also identified upon univariate GWA analysis (Figure 4.b). The SNP m33786 was associated with DW and was mapped to an intron of gene Di19. However, this SNP was in the intron but the Non-col like allele of SNP m33786 is more tolerant to the consecutive combination of Botrytis and PEG stress in comparison to the Col-0 like allele (Figure 5.e). When mapping the effect on WC for the same treatments, 43 associated SNPs were found, residing in 30 genes (Supplementary Figure 3.c and supplementary table 10). Four of these SNPs identify a region on $\mathrm{Chr} 3$ containing associated with seven genes with TAIR IDs At3g22670-At3g22730 (Supplementary Table 10). The combined boxplot of the haplotypes combining these four SNPs showed a that accessions containing the non-Col-0 allele did not exhibit differences in WC under the Botrytis and PEG treatment vs. the single Botrytis treatment than accessions containing the Col-0 allele (Supplementary 
Figure 4.a). In addition, six associated SNPs mapped to a region on Chr. 5 containing seven genes with TAIR IDs At5g48120-At5g48180. Boxplot of the haplotypes combining these six SNPs showed a that accessions containing the non-Col-0 allele did not exhibit differences in WC under the Botrytis and PEG treatment vs. the single PEG treatment than accessions containing the Col-0 allele (Supplementary Figure 4.b). Two SNPs m200317 and m200318 were residing in MET18 gene (At5g48120). No significant differences in WC between accessions carrying the non-Col-0 alleles of the two SNPs under the Botrytis and PEG treatment vs. the single PEG treatment when compared to accessions containing the Col-0 allele (Supplementary Figure 4.c). SNPs m200329, m200331 and m200335, residing in the genes with TARI IDs At5g48130 and At5g48140. Boxplot of the haplotypes combing the three SNPs showed that accessions containing Non-Col-0 allele exhibited higher WC under the Botrytis and PEG treatment vs. the single Botrytis treatment than accessions containing the Col-0 allele (Figure 5.h). SNP m200389, residing in NITRILE SPECIFIER PROTEIN 5 (ATNSP5) gene (At5g48180), the non-Col-0 allele did not show difference in relative WC upon Botrytis and PEG stress vs. PEG stress alone, when compared to accessions carrying the Col-0 allele (Supplementary Figure 4.d). SNP m60883 was identified by both univariate and ME GWA-mapping approaches. is from C (Col-0 like) to $\mathrm{G}$ (non-Col like) led to a nonsynonymous amino acid substitution from Glu to GIn (A-A position E500Q. The SNP is residing in gene with TAIR ID AT2G13690, and the non-Col-0 allele did not show difference in relative WC upon Botrytis and PEG stress vs. PEG stress alone, when compared to accessions carrying the Col-0 allele (Supplementary Figure 4.e)

\section{Amino acid substitution of the significant SNPs in response to combinatorial stress}

Using ME and univariate GWA mapping approaches revealed a number of QTLs containing candidate genes that could play a role in the response to combinatorial stresses. To support the relevance of the identified loci, several SNPs were found using both methods (Figure 6). This involves only a few loci though, for the response to Pieris and subsequent PEG the QTL on Chr 2 comprising genes with IDs At2g36550 and At2g36560, linked to eight associated 
SNPs and the QTL on Chr 3 around gene AT3G45910, linked to one associated SNP. For the response to Botrytis and PEG four common SNPs were found in both approaches, covering as many loci, around genes with IDs At1g48670, At1g56280 (DI19), At2g13690 and a larger region comprising At5g48120 At5g48180.

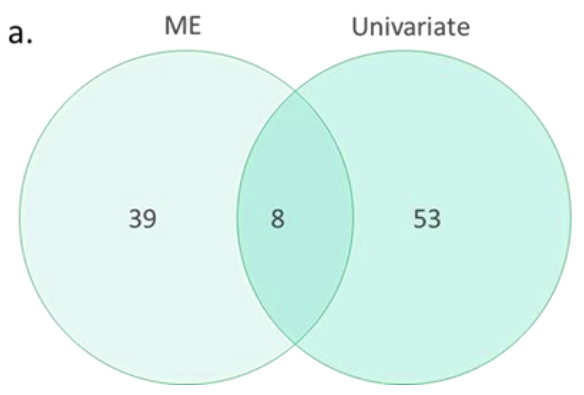

b. ME Univariate

Figure 6. Venn diagram exhibit significant SNPs identified by ME and univariate GWAmapping methods for traits related to the combined Pieris and subsequent PEG treatment response (a), and the Botrytis and subsequent PEG treatment response (b).

Although not all polymorphisms will be represented in the SNP markers used for GWAS analysis, some of the identified SNPS could be causal to the allelic variation. Therefore the allelic effect on any coding region was determined for these SNPS. SNP marker m73991 resides in At2g36550, encoding a Haloacid dehalogenase-like hydrolase (HAD) superfamily protein, with the allelic difference causing a non-synonymous amino acid substitution from an Asp to a Lys (A-A position N138K) residue in the non-Col-0 allele. Two additional SNPs, m73993 and m73994, cause non-synonymous changes to the coding region of At2g36560, encoding a protein of unknown function and the expression was very low, substituting Ala to Glu (A-A position A488E) and Glu to Gly (A-A position E420G) amino acids. SNP $\mathrm{m} 111770$ is residing in the intergenic region between genes with TAIR IDs At3g45910 and At3g45920, but is in LD $(r=0.53)$ with another SNP causing a Val to Ile (A-A position V44I) substitution in the coding region of At3g45920.

The allelic effect of SNP m33786 on DI19 gene was showed in Figure 4.e. SNP m60883 is residing in gene with TAIR ID AT2G13690. The coding sequence 
substitution of SNP m60883 is from C (Col-0 like) to G (non-Col like) led to a nonsynonymous amino acid substitution from Glu to Gln (A-A position E500Q).

\section{PRX34 and RMG1 play a role in the response of PLA to consecutive biotic and PEG stresses}

A number of genes in LD with the significant SNPs have reported roles in response to biotic or abiotic stresses, but not to Pieris, Botrytis, or PEG. To determine if they play a role in response to these stresses, mutants for seven genes associated with response QTLs were further examined (Table 1). The HAD gene family member At2g36560 (Pieris and PEG) and DI19 (Botrytis and PEG) were identified by both ME and univariate GWA-mapping approaches. In total, nine homozygous T-DNA insertion mutants, one for each of these nine candidate genes, were further analysed.

Plants were subjected to the same treatments as the HapMap accessions have been to (Figure 2). The di19 mutant exhibited small rosette in all conditions when compared to the wild type (Supplementary Figure 5). One-way ANOA was performed to test the significance of projected rosette area expansion of T-DNA insertion mutants to the wild type, it shows that the prx34 and bzip25 mutants were more tolerant to Pieris herbivory (Figure 7.a) and the rmg1 mutant was more tolerant to Botrytis infection when compared to the wild type (Figure 7.b). Two-way ANOVA was performed to test the significance of the response to PEG treatments compared to wild-type plants. When determining the response to single PEG, the prx34, di19 and rmg1 mutants were more tolerant than the wild type (Figure 7.c \& d). When regarding the combinatorial treatments, the prx34 mutant was found to be more sensitive to the combination of Pieris and subsequent PEG treatment (Figure 7.e); while the arf4 mutant was more tolerant to the combination of Botrytis and subsequent PEG treatment (Figure 7.f). 


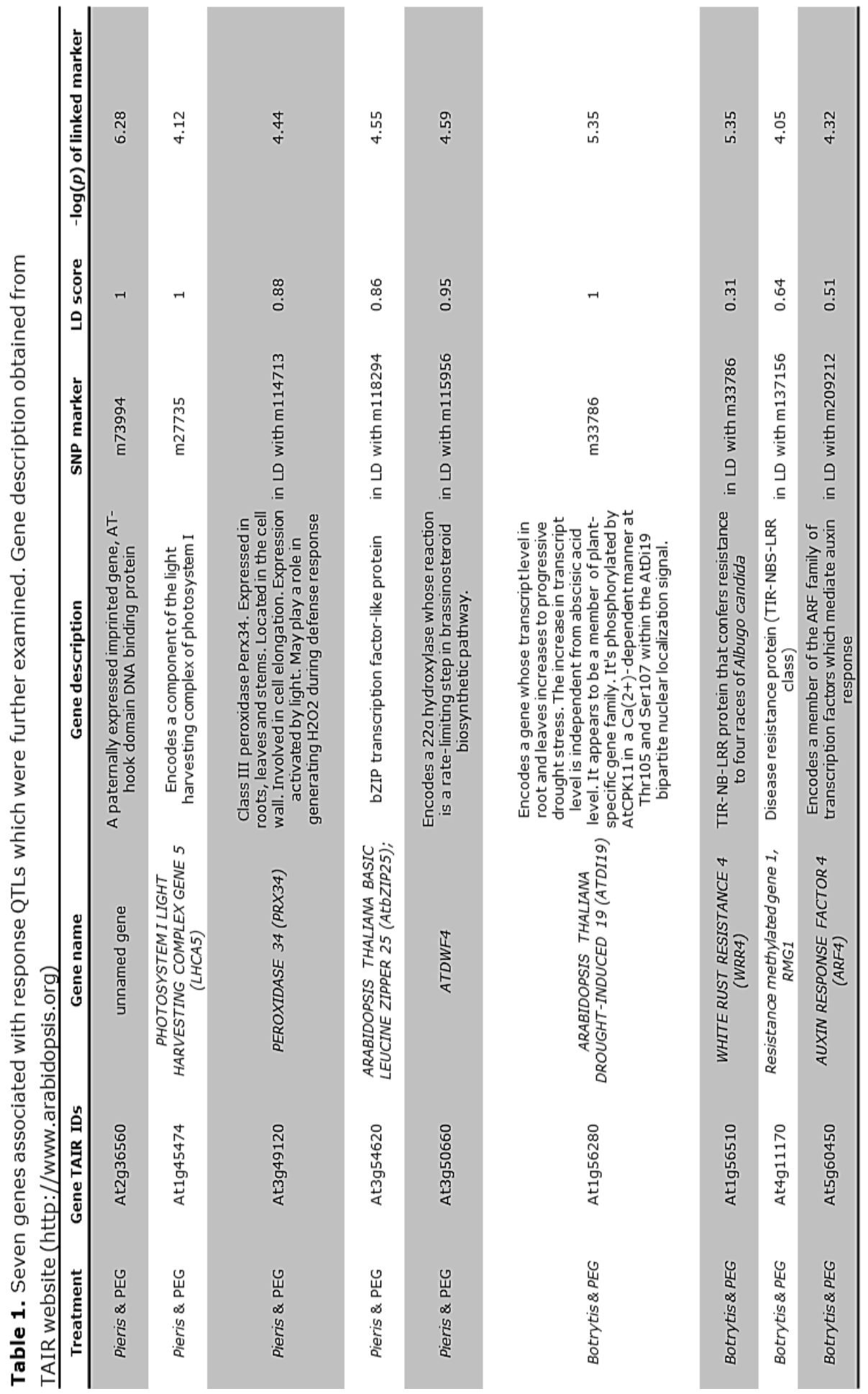


a.

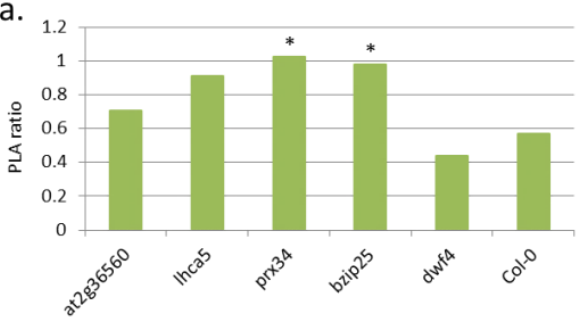

c.
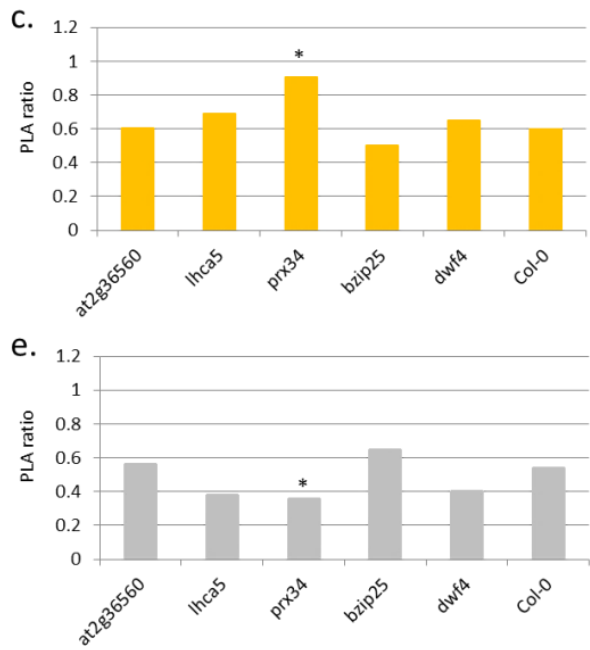
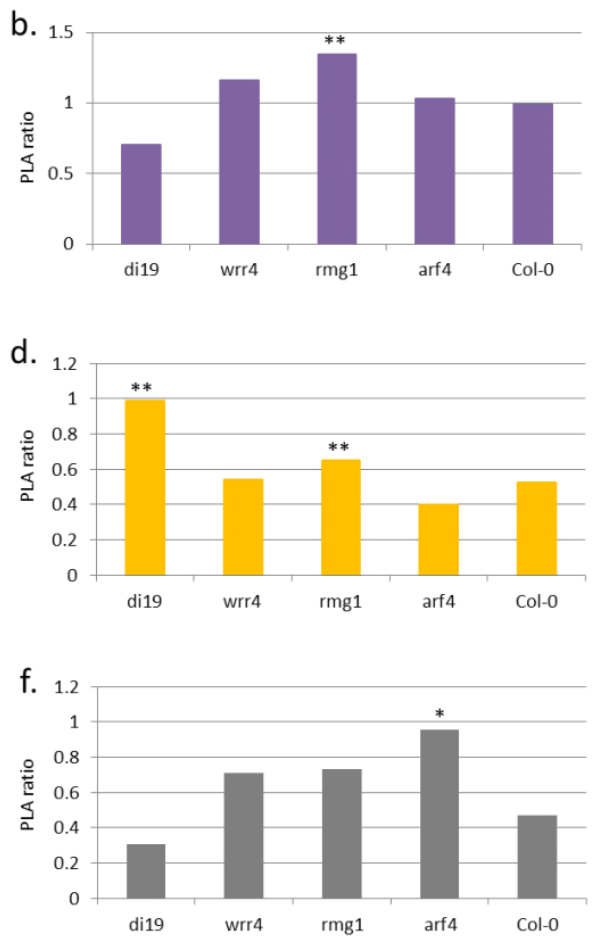

Figure7. Ratios of projected rosette areas (PLA) of T-DNA inerstion mutants Ihca5, prx34, bzip25, dwf4, wrr4, rmg1, arf4, di19 and for the HAD gene family member with TAIR ID At2g36560 subjected to several (a)biotic stress treatments, compared to Col-0 wild type.

a. Comparing plants treated with Pieris to the PLA of the same plants before treated with Pieris

b. Comparing plnats treated with Botrytis to the PLA of the same plants before treated with Botrytis

c. Ratio of PLA of the genotypes in figure 7.a after subjected to PEG, compared to the control (yellow).

d. Ratio of PLA of genotypes in figure 7.b after subjected to PEG, compared to the control (yellow).

e. Ratio of PLA of Col-0 wild type and the mutant plants after pre-treatment with Pieris and subsequent exposure to PEG, compared to plants exposed to PEG only (light grey).

f. Ratio of PLA of Col-0 wild type and the mutant plants after subjected to pretreatment with Botrytis and subsequent exposed to PEG, compared to plants exposed to PEG only (dark grey).

Two-way ANOVA was used for analysis of differences between mutants and wild type, samples comprised six plants per treatment per line. 


\section{The response of the extreme accessions to single and combined stresses}

Some accessions that exhibited a phenotype on the extreme ends of the spectrum (either more tolerant or more sensitive) to the combinatorial stress treatments were analysed for expression of the selected candidate genes upon exposure to different stress treatments. This involved four accessions responsive to Pieris and PEG and six accessions responsive to Botrytis and PEG (table 2).

Table 2. Accessions showed extreme phenotype in response to combinatorial stress from the result of screening Hapmap population

\begin{tabular}{llll}
\hline Treatment & CS code & Accessions & Phenotype \\
\hline Pieris \& PEG & CS28201 & Da(1)-12 & Tolerant \\
\hline Pieris \& PEG & CS76105 & Bur-0 & Tolerant \\
\hline Pieris \& PEG & CS76124 & Duk & Susceptible \\
Pieris \& PEG & CS76156 & Kulturen-1 & Susceptible \\
Botrytis \& PEG & CS76143 & Hovdala-2 & Tolerant \\
Botrytis \& PEG & CS76147 & In-0 & Tolerant \\
Botrytis \& PEG & CS76164 & Ler-1 & Tolerant \\
Botrytis \& PEG & CS76214 & Pro-0 & Susceptible \\
Botrytis \& PEG & CS76218 & Rennes-1 & Susceptible \\
Botrytis \& PEG & CS28743 & Sp-0 & Susceptible \\
\hline
\end{tabular}

Different responses to the combinatorial stress was found when compared to the result from screening the Hapmap accessions, Kulturen-1 was tolerant to the Pieris \& PEG combined stress when compare to single stress (Figure 8.a), Ler-1 was susceptible to Botrytis \& PEG when compared to single PEG while Rennes- 1 and Sp-0 were tolerant to the combinatorial stress (Figure 8.b). No significant correlation between the result from the screening Hapmap population and the re-screen data, showing a variation of the environmental factors between the two times screening. Significant interaction between treatments and accessions were observed on DW (Supplementary Table 12). 


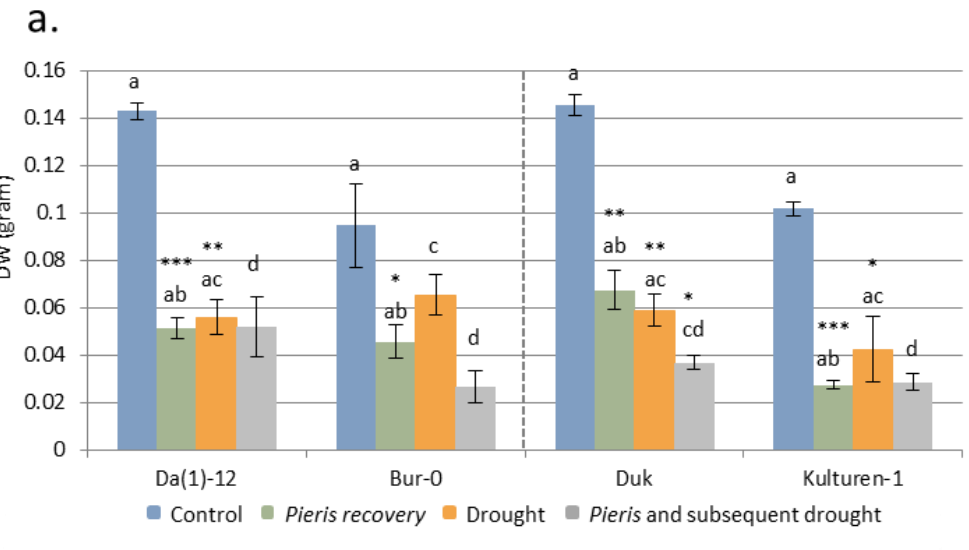

b.

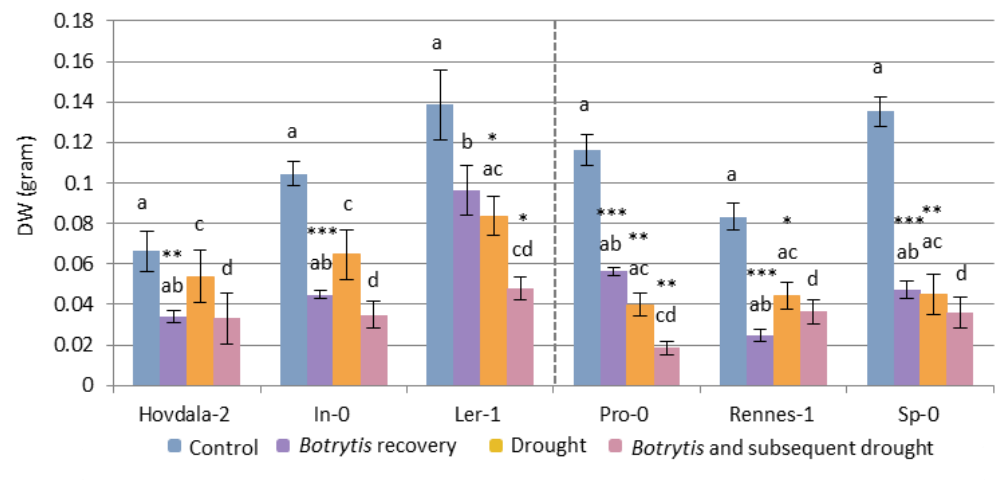

Figure 8. Phenotypes of Arabidopsis accessions $\mathrm{Da}(1)-12$, Bur-0 (more tolerant), Duk and Kulturen-1 (more sensitive), identified as extremes in the response to the Pieris and subsequent PEG treatment, and Hovdala-2, In-0, Ler-1 (more tolerant), Pro-0, Rennes-1 and Sp-0 (more sensitive), identified as extremes in the response to the Botrytis and subsequent PEG treatment. Vertical dashed line separate accessions were either tolerant or susceptible to combinatorial stress (result from screening the HapMap population).

a. Rosette dry weights (DW) of accessions grown in control conditions (blue), after Pieris pre-treatment (green), after PEG treatment (yellow), and after the combinatorial Pieris and PEG treatment (grey).

b. DW of accessions grown in control conditions (blue), after Botrytis pretreatment (purple), after PEG treatment (yellow), and after the combinatorial Botrytis and PEG treatment (lilac).

Two-way ANOVA was used to compare accessions under single Pieris and single PEG to the control, or under the combinatorial treatment to the PEG treatment. * indicates mutants with a significant difference under single stress in comparison to the control, or under combinatorial stress in comparison to single PEG $(p<0.05$, $* * p<0.01, * * *<0.001)$. Averages $\pm \mathrm{SE}$ are shown, $\mathrm{n}=4$. 


\section{Variation in candidate gene expression in extreme accessions grown under different stress conditions}

The expression of the candidate genes bZIP25, PRX33 (in LD with PRX34), PRX34, LHCA5, DWF4 was determined in the accessions Duk, Kulturen-1, Da(1)12, and Bur-0, grown under control, Pieris, PEG, and Pieris and subsequent PEG treatments (Figure 9). Three-way ANOVA to test the interaction between accessions and treatment was performed for bZIP25, PRX33, and LHCA5 exhibited significant interaction between accessions, Pieris, and PEG (Supplementary Table 13). The five genes were downregulated in all accessions grown under PEG and combinatorial stresses. The expression under Pieris treatment exhibit contrasting expression direction when compared to the PEG treatment. In general, all genes were down-regulated under single PEG and the combinatorial stress. bZIP25 is more down-regulated in Kulturen-1 than other accessions under PEG treatment, the expression is less down-regulated in Bur-0 under combinatorial stress when compared to single PEG treatment (Figure 9.a). $P R X 33$ was less down-regulated in $\mathrm{Da}(1)-12$ than other accessions under PEG treatment, the expression is more down-regulated in $\mathrm{Da}(1)-12$ but less downregulated in Bur-0 under combinatorial stress in compression to single PEG stress (Figure 9.b). PRX34 is less down-regulated in $\mathrm{Da}(1)-12$ and Duk than in Bur- 0 and Kulturen-1, the expression was more down-regulated under the Pieris and PEG combined stress when compared to single PEG (Figure 9.c). LHCA5 was extreme down-regulated under PEG treatment but less down-regulated under the combinatorial treatment when compared to single PEG (Figure 9.d). DWF4 is less down-regulated than other accessions under PEG treatment, reduced downregulation was observed in Bur-0 and Kulturen-1 under Pieris combined stress when compared to single PEG treatment (Figure 9.e).

The expression levels of the DI19, RMG1, ARF5, and WRR4 genes in response to the Botrytis treatment and the subsequent PEG conditions was studied in the 6 accessions; Pro-0, Rennes-1, Sp-0, Hovdala-2, In-0, and Ler-1 (Figure 10). Three-way ANOVA to test the interaction between treatments and accessions of RMG1 and WRR4 showed significant interaction between accessions, Botrytis, and PEG treatments (Supplementary Table 14). Strong 
expression of RMG1 was observed in Botrytis infection condition, the expression fold change up to 256 times relative expression fold change in accession Sp-0 (Figure 10.b). Under PEG stress, DI19 and WRR4 were upregulated in all accessions (Figure 10.a \& d). variations of DI19 and ARF4 were observed among accessions; DI19 was up-regulated up to 4 times fold change in Hovdala-2 and ARF4 was up-regulated up to 8 times fold change in the same accession. Under combinatorial stress, all accessions exhibit down-regulation ARF4, except in Rennes-1 (Figure 10.c). Variation of RMG1 expression was found among accessions. The gene is down-regulated in Hovdala-2 but up-regulated in Sp-0 under combinatorial stress when compared to single PEG treatment.
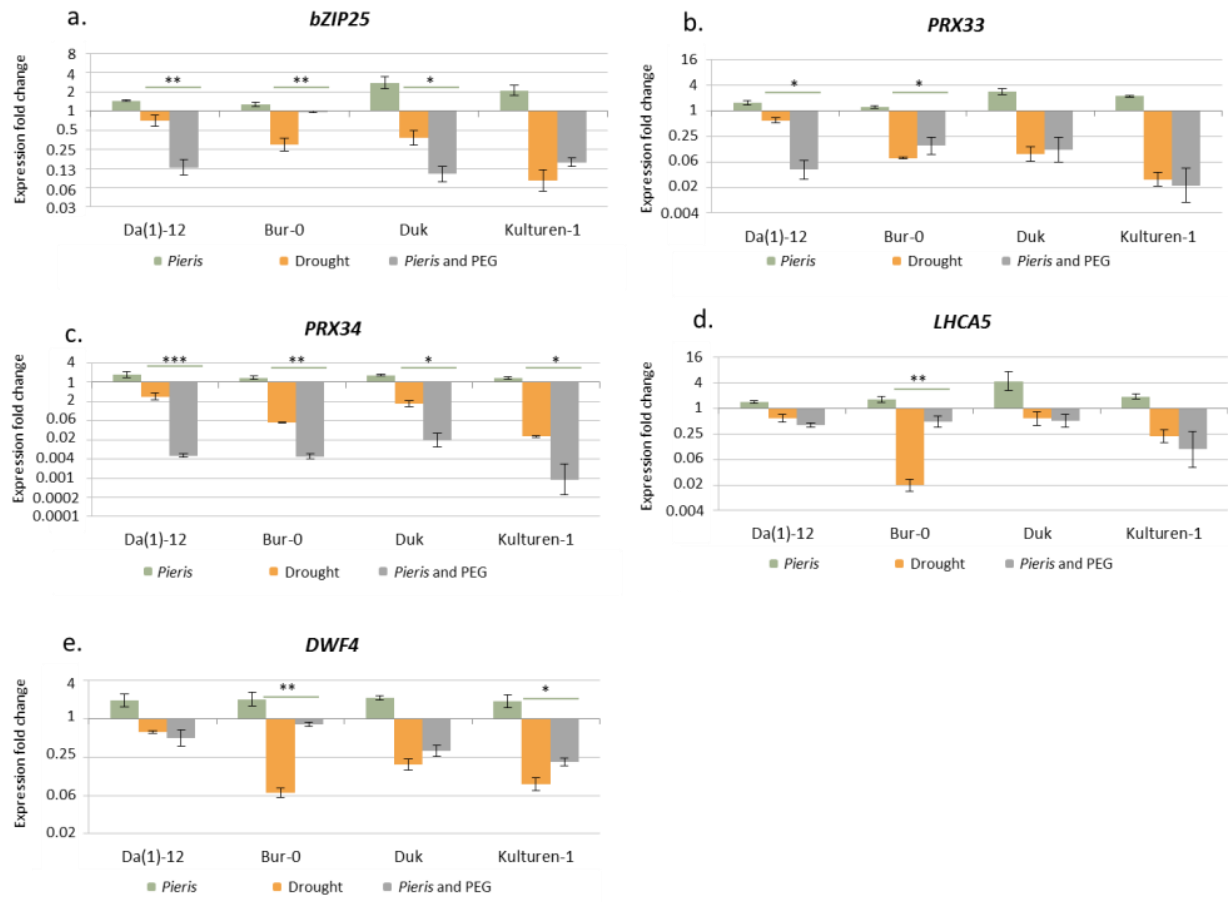

Figure 9. Relative rosette gene expression analysis of $b Z I P 25$ (a.), $P R X 33$ (b.), $P R X 34$ (c.), LHCA5 (d.), and DWF4 (e.) in four Arabidopsis accessions selected based on extreme phenotypes, after Pieris treatment (green), PEG treatment (yellow), and the combinatorial Pieris and subsequent PEG treatment (grey), compared to the expression under control, untreated, conditions. Two-way ANOVA was used to compare gene expressions under combinatorial stress to PEG for each accession. Average rel. expression differences observed in treatments compared to control conditions are shown $\pm \mathrm{SE} ; \mathrm{n}=3$. * indicates a significant difference of gene expression under combinatorial stress when compared to the PEG $(* p<0.05, * * p<0.01, * * * p<0.001)$ 

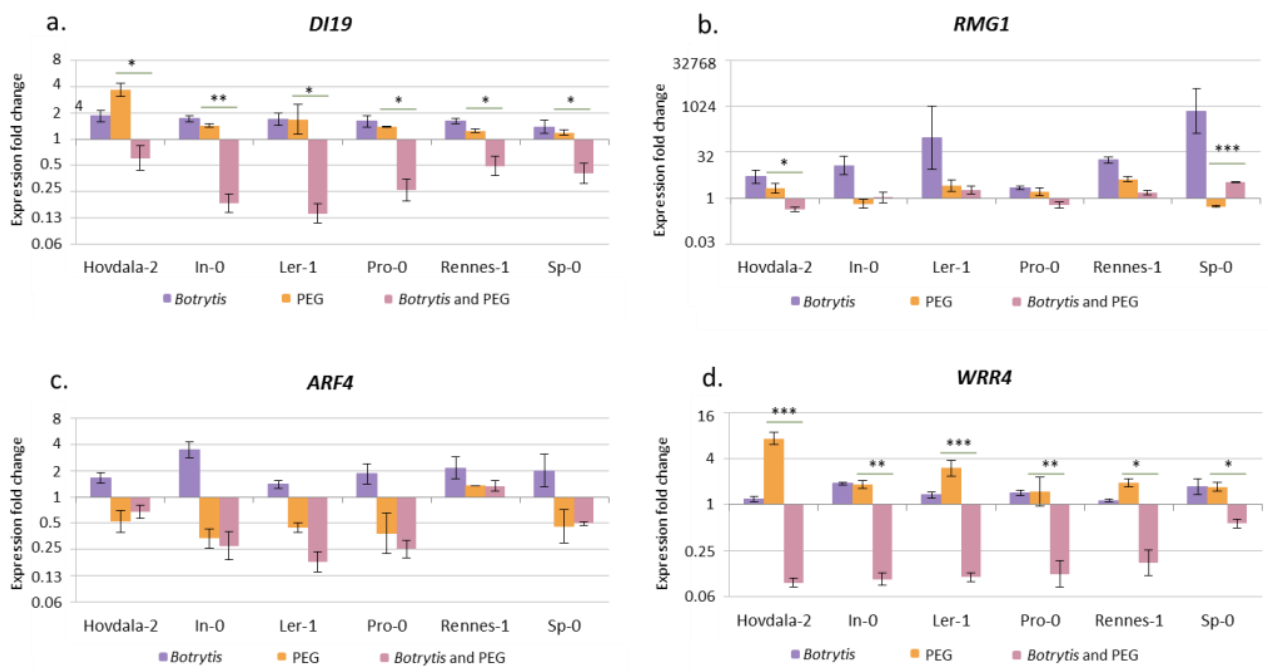

Figure 10. Relative rosette gene expression analysis of DI19 (a.), RMG1 (b.), ARF4 (c.), and WRR4 (d.) in four Arabidopsis accessions selected based on extreme phenotypes, after Botrytis treatment (Purple), PEG treatment (yellow), and the combinatorial Botrytis and subsequent PEG treatment (Pink), compared to the expression under control, untreated, conditions. Two-way ANOVA was used to compare gene expressions under combinatorial stress to PEG within each accession. Average rel. expression differences observed in treatments compared to control conditions are shown $\pm S E ; n=3$. * indicates a significant difference of gene expression under combinatorial stress when compared to the PEG $(* p<0.05, * * p<0.01, * * * p<0.001)$ 


\section{Discussion}

\section{Comparing the PEG osmotic stress treatment with conventional drought stress}

PEG8000 was used to simulate drought stress. PEG and conventional drought by soil-drying is known to cause some differences in plant responses, such as for instance shown by differences in proline accumulation and leaf thickness in apple, while the impacts on Relative Water Content (RWC) and chlorophyll content are similar (Kautz et al., 2015). We expected that a PEG treatment would emphasize the osmotic aspects of drought and thus more the physiology related to drought tolerance, rather than plant architectural responses contributing to drought avoidance, like for example adaptations of the root system architecture (El-Soda et al., 2015). The latter would be much less expressed in our rock wool-based growth system anyhow, since the small rock wool cubes do not allow for root drought avoidance. The mean reason to revert to a PEG treatment was related to the difficulties we experienced in obtaining homogeneous drought on peat-based substrates for a large set of plants, which would be needed for a proper GWA analysis of the Arabidopsis HapMap population. We are aware of much more suitable systems, especially designed to screen for plant drought on soil, such as the Phenopsis system (Granier et al., 2006), but logistic issues made us instead decide to use the PEG-mediated osmotic stress. There is also an important advantage of such approach, as we could use this in our rock wool based growth facilities equipped with imaging systems to record plant size and thus accurately measure plant growth (BacMolenaar et al., 2016; Flood et al., 2016). Using an ebb-flood system to water the rock wool blocks also allowed us to reproducibly test different PEG-nutrient solution mixtures for the most suitable osmotic potential for screening. In order to verify that the PEG-nutrient solution induced similar, but more controlled, symptoms as observed upon conventional drought stress of plants raised on peat-based mixtures by withholding watering, we examined gene expression of a set of known drought responsive genes in both conditions and their respective controls (Figure 3). The five tested genes are involved in the ABA-dependent and -independent regulatory system for drought responses (Shinozaki and Yamaguchi-Shinozaki, 2007). Since we observed comparable expression profiles 
of these drought-responsive genes in response to PEG and soil-drying treatments, we concluded that the PEG8000 induced osmotic stress would be sufficiently suitable to study the Arabidopsis 'drought' response.

\section{Allelic effects on combinatorial stress responses}

Previous studies of Arabidopsis natural variation exhibited different effects of allelic variation on plant morphology and stress responses which led to the identification of the gene involved. For instance, GWA mapping of cellular traits identified the KUK gene involved in root development regulation and root growth (Meijon et al., 2014). Likewise, different alleles of the ACCELERATED CELL DEATH 6 (ACD6) gene were found to exhibit pleiotropy effects on pathogen resistance and results in plants with small rosettes (Todesco et al., 2010). In general, an allelic effect of a locus can be due to a single bp mutation, such as often found in EMS-mutagenized populations, which can be simple SNPs, for instance when such a SNP causes a non-synonymous amino acid substitution in one of the exons of the gene involved or when it causes an intron-exon splice junction error (Brown, 1996; Guyon-Debast et al., 2010). Both cases are likely to affect the amino acid sequence of the protein encoded by the gene, which can affect its function. For instance, a SNP causing alternative splicing of the FLOWERING LOCUS C (FLC) antisense transcript COOLAIR, resulted in differential expression of $F L C$ and altered flowering time in Arabidopsis ( $L i$ et al., 2015). Furthermore, natural accessions carrying a single SNP at the $\mathrm{HIGH}$ AFFINITY K+ TRANSPORTER $1 ; 1($ AtHKT1;1) gene affected the expression of the gene leading to a difference in leaf $\mathrm{Na}^{+}$accumulation (Baxter et al., 2010).

In this study, we initially identified SNPs as markers for the traits we used to evaluate the response to single and combinatorial stresses. Of the two GWA approaches we employed, the univariate approach yielded more associated SNPs than the ME approach. This could be due to a higher number of false positives in univariate approach, which is known to occur when compared to the ME approach, as the latter also considers the within and the between trait(s) variation (Korte et al., 2012). The traits we measured (FW, DW, WC and PLA) are very general growth traits, likely to be genetically affected by allelic variation at many loci. So, even when heritabilities are high, which was the case for these traits (table 2), the multitude of small-effect QTLs could make it very difficult to 
identify these QTLs (Kooke et al., 2016). This is also the reason we used an arbitrary threshold of $-\log (p)=4$ to assign associated SNPs (van Rooijen et al., 2015), instead of the much more conservative Bonferroni corrected threshold ($\log (p)=6.5$ ) which is advised (Atwell et al., 2010). If this conservative threshold was used, we would only consider one associated SNP for the univariate GWA approach, m73995, close to gene ID At2g36550, for rosette PLA upon combinatorial Pieris and PEG stress (Supplementary Figure 2.a and supplementary Table 8). This same SNP was also identified in the ME GWA approach (Supplementary Figure 3.a and supplementary Table 10). For the response to Botrytis and PEG, only the ME GWA approach would have yielded significantly associated SNPs; m64241, close to the GENE WITH UNSTABLE TRANSCRIPT 15 (At2g18440); and m94602, close to an unnamed gene (At3g22690) involved in chloroplast RNA editing, and in LD with a region containing seven more genes (At3g22670-At3g22740), of which RNA-DIRECTED DNA METHYLATION 1 (RDM1), involved in DNA methylation and YELLOW SEEDLING 1 (YS1), involved in chloroplast RNA editing, have been analysed in some detail (Zhou et al., 2009; Law et al., 2010).

To avoid false positives, we focussed on genes identified in both approaches for further analysis, as we considered those to be less likely to be false positive. The genetically best supported locus comprises two unnamed genes (At2g36550 and At2g36560), supposedly involved in the response to Pieris and PEG. There are several SNPs in LD with the main associated SNP, all residing in the At2g36560 gene, which could explain the allelic variation. Unfortunately the KO mutant for this gene did not show any aberrant responses to the stress treatments in comparison to wild-type plants to support it as the most likely candidate underlying the allelic variation. It could be that the Col-0 allele is redundant, but that other accessions carry gain-of-function allelic variants affecting the phenotype. At2g36550 is a member of Haloacid dehalogenase-like hydrolase (HAD) superfamily protein and At $2 \mathrm{~g} 36560$ is a member of AT hook domain DNA-binding protein. The biological functions of the two genes haven't yet to be studied. However, At2g36560 could act as a positive factor for gene expression. For instance, one of the AT hook motif DNA binding protein (PF1) 
was found can bind to the promoter site of oat photochromic A3 (PHYA3) gene and could has influence on the gene expression (Nieto-Sotelo et al., 1994).

In case of DW measured upon Botrytis and subsequent PEG stress, both mapping approaches identified SNP marker m33786 to be associated. This marker resides in an intron of the DI19 gene, but in LD with this marker there are three more genes: an unnamed gene encoding a CwfJ-like Zn-finger DNA binding protein, that is expressed in response to Cabbage leaf curl virus (CaLcuV) infection (Ascencio-Ibanez et al., 2008) (At1g56290); an unnamed gene which encodes a chaperone DnaJ-domain superfamily protein, expressed in response to oxidative stresses (At1g56300); and an unnamed gene that encodes a ribonuclease $\mathrm{H}$-like superfamily protein (At1g56310). Although no SNP in any of these gene could be identified that would explain the allelic variation, the DI19 gene is the most likely candidate underlying this QTL, as in our hands, the KO mutant was very small, under all tested conditions (Supplementary Figure 5), and we could not test it properly for response to stress treatments. The expression of Di19 can be found in seedlings, roots, rosettes, stems, flowers, and siliques (Milla et al., 2006). The gene is up-regulated under drought, but did not respond to ABA (Gosti et al., 1995), it is down-regulated under Botrytis infection, and the combination of Botrytis and subsequent drought (Coolen et al., 2016). Liu et al. (2013) reported that the di19 knockout mutant was susceptible to drought and that the constitutive overexpression line was tolerant to drought. The gene encodes a Cys2/His2-type zinc-finger protein that functions as a transcription factor (Liu et al., 2013). The expression of DI19 can be modulated by Calcium-dependent Protein Kinases CPK3, CPK4, CPK11, and CPK12 via $\mathrm{Ca}^{+}$-dependent signalling (Milla et al., 2006; Rodriguez Milla et al., 2006). Some of the CPK family proteins were reported to be involved in biotic stress resistances. For instance, CPK1 plays a role in Botrytis, Fusarium oxysporum, and Pseudomonas syringae pv.tomato DC3000 (Pst) resistance (Coca and San Segundo, 2010), while CPK4, CPK5, CPK6, and CPK11 are involved in Pst resistance (Boudsocq et al., 2010), CPK5, CPK6, and CPK11 are involved in Botrytis resistance (Gravino et al., 2015), and CPK3 and CPK13 act as positive regulators of PDF1.2 resistant to Spodoptera littoralis caterpillars (Kanchiswamy et al., 2010). Thus, we hypothesize that the down-regulation of DI19 under Botrytis infection is due to the negative regulation by CPKs. 


\section{Role of other candidate genes in response to single and combinatorial stresses}

Some candidate genes in LD with the significant SNPs have been reported to have a role in the response to stresses, but not yet in the response to Pieris, Botrytis, and PEG. We therefore identified homozygous T-DNA insertion mutants for as many of these genes and determined their phenotypes in response to treatments.

Mutant prx34 was found to be more susceptible to the Pieris and PEG combinatorial stress (Figure 7). The univariate GWA-mapping on PLA showed another gene, $P R X 33$ (AT3G49110), to be in LD with $P R X 34$. Both genes encode cell-wall peroxidases (Valerio et al., 2004). PRX34 is more abundantly expressed than PRX33 (Sultana et al., 2015), and the expression of $P R X 33$ is partially controlled by $P R X 34$ (Daudi et al., 2012). Knockout mutant prX34 was found to exhibit reduced expression of both $P R X 33$ and $P R X 34$ and reduced 50\% MAMPinduced ROS production in apoplastic space (Daudi et al., 2012; O'Brien et al., 2012). The double knock-out mutant prx33prx34 expressed a number of Pierisresponsive genes such as VSP2, which were not expressed in the wild type (Liu et al., 2005; Dombrecht et al., 2007; Mammarella et al., 2014), as well as other JA and ET-responsive genes such as VSP, PR3, PR4 (Mammarella et al., 2014). It is possible that the resistance of the prx34 mutant to Pieris is due to the enhanced expression of VSP2. The prx33prx34 double knockout mutant also showed reduced expression of drought tolerance gene ETHYLENE RESPONSE FACTOR 1 (ERF1) (Cheng et al., 2013; Mammarella et al., 2014), which was negatively regulated by Pieris (De Vos et al., 2006). This could be the reason why the prx34 mutant was more sensitive to the consecutive Pieris and PEG stress combination instead of more tolerant as expected based on the response to Pieris alone.

RMG1 encodes a NB-LRR-TIR protein and the promoter region contains two helitron-related repeats AtERP4 and AtERP11, which are the target by siRNAs and can be heavily methylated in all cytosine sequencing contexts ( $Y u$ et al., 2013). It is a primary target of the RNA-directed DNA methylation (RdDM) and the expression can be enhanced by flg22 treatment in the knockout mutant of 
gene Repressor Of Silencing 1 (ROS1) (Yu et al., 2013). Thus, the observed phenotype of rmg1 mutants in response to stresses could be due to the RMG1 involved RdDM. In addition, the body of the defense responsive gene WRKY22 was found at the $5^{\prime}$ part of the open reading frame ORF of $R M G 1$ ( $Y u$ et al., 2013), which suggests a epigenetic control of the two genes under multiple stresses.

When looking at the effect of biotic pre-treatment on plants in response to PEG, we found the Pieris pre-treatment has positive effect on prx34 mutant and the Botrytis pre-treatment has positive influence on $r m g 1$ mutant in response to PEG stress (Figure 7). Comparing candidate genes expression under the combinatorial stress to PEG stress, the expression of $P R X 34$ is more downregulated under the combinatorial stress when compared to single PEG stress (Figure 9). Negative effects of Botrytis pre-treatment was observed on DI19 gene expression in response to drought stress (Figure 10). We hypothesised the interaction between biotic and abiotic stress responses can be synergistic, antagonistic, or neutral. In this study, additive effects was found on arf4 mutant, which did not show significant responses to individual Botrytis and PEG treatments but was tolerant to the combination. Antagonistic effect was observed on prx34 mutant, the mutant was tolerant to Pieris and PEG stresses, but was susceptible to the combination of Pieris and PEG when compared to the wild type (Figure 7). Neutral effect was observed on rmg1 mutant, the mutant was tolerant to individual Botrytis and PEG treatments, but did not show different phenotype under the combination of Botrytis and PEG treatment when compared to the wild type. This suggests it will be impossible to obtain genotypes that would be tolerant to both single and combinatorial stresses. To illustrate this, overexpression of $P R X 34$ and ARF4 could result in enhanced tolerance to combinatorial stresses, but this is likely to reduce the tolerance to each of the biotic single stresses. 


\section{Conclusion}

To conclude, this study used two GWA-mapping approaches and identified a number of candidate genes exhibited allelic variation in the response to combinatorial stress. Genes. To confirm the allelic effect on stress responses, genetic complementation tests to examine the biological function of the different alleles in response to different stress conditions will be needed. We validated the biological function of nine genes, of which seven genes were in LD with the identified associated SNPs, not the gene in which the SNP resides. These genes have reported functions in response to some stresses, in single and combinatorial stress conditions. Antagonistic interaction between Pieris and PEG was observed for the prx34 mutant, and antagonistic interaction between Botrytis and PEG for the rmg1 mutant. The expression of candidate genes in single and combinatorial conditions was measured from Arabidopsis accessions. However, we did not observe a relation between the variation of the phenotypic responses in Arabidopsis natural accessions and the variation in candidate gene expression. A powerful analysing method such as algorithm locally linear embedding graph generator (LEGG) (Van Poecke et al., 2007) could be useful to define relationships between expression profile and phenotypic variances in response to stresses. 


\section{Acknowledgement}

We thank Taede Stoker, Gerrit Stunnenberg, and Pauline Sanderson of Unifarm, Wageningen UR, for their help with plant propagation. We acknowledge Dr. Yifang Chen (China Agriculture University, PR China) for providing seeds of the di19 mutant. We are grateful to the Entomology group of Wageningen University for providing us with Pieris rapae larvae, and the Plant-Microbe Interactions group of Utrecht University and the Phytopathology group of Wageningen University for providing us with Botrytis cinerea cultures. We thank Prof. Maarten Koornneef and Dr. Robert Akkers for critical reading of the manuscript and numerous very useful suggestions. This work was financially supported by The Netherlands Organization for Scientific Research (NWO) through the Technology Foundation, Perspective Programme 'Learning from Nature' [STW10988]. 


\section{References}

Achuo EA, Prinsen E, Höfte M (2006) Influence of drought, salt stress and abscisic acid on the resistance of tomato to Botrytis cinerea and Oidium neolycopersici Plant Pathology Volume 55, Issue 2. In Plant Pathology, Vol 55, pp 178-186

Appel HM, Maqbool SB, Raina S, Jagadeeswaran G, Acharya BR, Hanley JC, Miller KP, Hearnes L, Jones AD, Raina R, Schultz JC (2014) Transcriptional and metabolic signatures of Arabidopsis responses to chewing damage by an insect herbivore and bacterial infection and the consequences of their interaction. Frontiers in Plant Science 5: 441

Ascencio-Ibanez JT, Sozzani R, Lee TJ, Chu TM, Wolfinger RD, Cella R, Hanley-Bowdoin L (2008) Global analysis of Arabidopsis gene expression uncovers a complex array of changes impacting pathogen response and cell cycle during geminivirus infection. Plant Physiol 148: 436-454

Atkinson NJ, Urwin PE (2012) The interaction of plant biotic and abiotic stresses: from genes to the field. Journal of Experimental Botany 63: 3523-3543

Atkinson NJ, Urwin PE (2012) The interaction of plant biotic and abiotic stresses: from genes to the field. J Exp Bot 63: 3523-3543

Atwell S, Huang YS, Vilhjalmsson BJ, Willems G, Horton M, Li Y, Meng D, Platt A, Tarone AM, Hu TT, Jiang R, Muliyati NW, Zhang X, Amer MA, Baxter I, Brachi B, Chory J, Dean C, Debieu M, de Meaux J, Ecker JR, Faure N, Kniskern JM, Jones JD, Michael T, Nemri A, Roux F, Salt DE, Tang C, Todesco M, Traw MB, Weigel D, Marjoram P, Borevitz JO, Bergelson J, Nordborg M (2010) Genome-wide association study of 107 phenotypes in Arabidopsis thaliana inbred lines. Nature 465: 627631

Bac-Molenaar JA, Fradin EF, Becker FF, Rienstra JA, van der Schoot J, Vreugdenhil D, Keurentjes JJ (2015) Genome-Wide Association Mapping of Fertility Reduction upon Heat Stress Reveals Developmental Stage-Specific QTLs in Arabidopsis thaliana. Plant Cell 27: 1857-1874

Bac-Molenaar JA, Granier C, Keurentjes JJ, Vreugdenhil D (2016) Genomewide association mapping of time-dependent growth responses to moderate drought stress in Arabidopsis. Plant Cell Environ 39: 88-102

Baxter I, Brazelton JN, Yu D, Huang YS, Lahner B, Yakubova E, Li Y, Bergelson J, Borevitz JO, Nordborg M, Vitek O, Salt DE (2010) A coastal cline in sodium accumulation in Arabidopsis thaliana is driven by natural variation of the sodium transporter AtHKT1;1. PLoS Genet 6: e1001193

Boudsocq M, Willmann MR, McCormack M, Lee H, Shan L, He P, Bush J, Cheng S-H, Sheen J (2010) Differential innate immune signalling via Ca2+ sensor protein kinases. 464: 418-422

Brown JW (1996) Arabidopsis intron mutations and pre-mRNA splicing. Plant J 10: $771-780$

Chao DY, Silva A, Baxter I, Huang YS, Nordborg M, Danku J, Lahner B, Yakubova E, Salt DE (2012) Genome-wide association studies identify heavy metal ATPase 3 as the primary determinant of natural variation in leaf cadmium in Arabidopsis thaliana. PLoS Genet 8: e1002923

Cheng MC, Liao PM, Kuo WW, Lin TP (2013) The Arabidopsis ETHYLENE RESPONSE FACTOR1 regulates abiotic stress-responsive gene expression by binding to different cis-acting elements in response to different stress signals. Plant Physiol 162: 1566-1582

Coca M, San Segundo B (2010) AtCPK1 calcium-dependent protein kinase mediates pathogen resistance in Arabidopsis. Plant J 63: 526-540

Coolen S, Proietti S, Hickman R, Davila Olivas NH, Huang PP, Van Verk MC Van Pelt JA, Wittenberg AH, De Vos M, Prins M, Van Loon JJ, Aarts MG, Dicke M, Pieterse CM, Van Wees SC (2016) Transcriptome dynamics of Arabidopsis during sequential biotic and abiotic stresses. Plant J 
Daudi A, Cheng Z, O'Brien JA, Mammarella N, Khan S, Ausubel FM, Bolwell GP (2012) The apoplastic oxidative burst peroxidase in Arabidopsis is a major component of pattern-triggered immunity. Plant Cell 24: 275-287

De Vos M, Van Zaanen W, Koornneef A, Korzelius JP, Dicke M, Van Loon LC, Pieterse CMJ (2006) Herbivore-Induced Resistance against Microbial Pathogens in Arabidopsis. Plant Physiology 142: 352-363

Dodds PN, Rathjen JP (2010) Plant immunity: towards an integrated view of plant-pathogen interactions. Nat Rev Genet 11: 539-548

Dombrecht B, Xue GP, Sprague SJ, Kirkegaard JA, Ross JJ, Reid JB, Fitt GP, Sewelam N, Schenk PM, Manners JM, Kazan K (2007) MYC2 differentially modulates diverse jasmonate-dependent functions in Arabidopsis. Plant Cell 19: 22252245

El-Soda M, Kruijer W, Malosetti M, Koornneef M, Aarts MG (2015) Quantitative trait loci and candidate genes underlying genotype by environment interaction in the response of Arabidopsis thaliana to drought. Plant Cell Environ 38: 585-599

Filiault DL, Maloof JN (2012) A genome-wide association study identifies variants underlying the Arabidopsis thaliana shade avoidance response. PLoS Genet 8: e1002589

Flood PJ, Kruijer W, Schnabel SK, van der Schoor R, Jalink H, Snel JF, Harbinson J, Aarts MG (2016) Phenomics for photosynthesis, growth and reflectance in Arabidopsis thaliana reveals circadian and long-term fluctuations in heritability. Plant Methods 12: 14

Gosti F, Bertauche N, Vartanian N, Giraudat J (1995) Abscisic acid-dependent and -independent regulation of gene expression by progressive drought in Arabidopsis thaliana. Mol Gen Genet 246: 10-18

Granier C, Aguirrezabal L, Chenu K, Cookson SJ, Dauzat M, Hamard P, Thioux JJ, Rolland G, Bouchier-Combaud S, Lebaudy A, Muller B, Simonneau T, Tardieu F (2006) PHENOPSIS, an automated platform for reproducible phenotyping of plant responses to soil water deficit in Arabidopsis thaliana permitted the identification of an accession with low sensitivity to soil water deficit. New Phytol 169: 623-635

Gravino M, Savatin DV, Macone A, De Lorenzo G (2015) Ethylene production in Botrytis cinerea- and oligogalacturonide-induced immunity requires calcium-dependent protein kinases. Plant J 84: 1073-1086

Guyon-Debast A, Lecureuil A, Bonhomme S, Guerche P, Gallois JL (2010) A SNP associated with alternative splicing of RPT5b causes unequal redundancy between RPT5a and RPT5b among Arabidopsis thaliana natural variation. BMC Plant Biol 10: 158

Hirt H (2009) Plant Stress Biology: From Genomics to Systems Biology. Wiley

Hirt H, Shinozaki K (2004) Plant Responses to Abiotic Stress. Springer

Kanchiswamy CN, Takahashi H, Quadro S, Maffei ME, Bossi S, Bertea C, Zebelo SA, Muroi A, Ishihama N, Yoshioka H, Boland W, Takabayashi J, Endo Y, Sawasaki T, Arimura G (2010) Regulation of Arabidopsis defense responses against Spodoptera littoralis by CPK-mediated calcium signaling. BMC Plant Biol 10: 97

Kang HM, Sul JH, Service SK, Zaitlen NA, Kong SY, Freimer NB, Sabatti C, Eskin E (2010) Variance component model to account for sample structure in genomewide association studies. Nat Genet 42: 348-354

Kautz B, Noga G, Hunsche M (2015) PEG and drought cause distinct changes in biochemical, physiological and morphological parameters of apple seedlings. Acta Physiologiae Plantarum 37: 1-6

Kooke R, Kruijer W, Bours R, Becker F, Kuhn A, van de Geest H, Buntjer J, Doeswijk T, Guerra J, Bouwmeester H, Vreugdenhil D, Keurentjes JJ (2016) Genome-Wide Association Mapping and Genomic Prediction Elucidate the Genetic Architecture of Morphological Traits in Arabidopsis. Plant Physiol 170: 2187-2203

Korte A, Vilhjalmsson BJ, Segura V, Platt A, Long Q, Nordborg M (2012) A mixed-model approach for genome-wide association studies of correlated traits in structured populations. Nat Genet 44: 1066-1071

Kruijer W, Boer MP, Malosetti M, Flood PJ, Engel B, Kooke R, Keurentjes JJB, van Eeuwijk FA (2015) Marker-Based Estimation of Heritability in Immortal Populations. Genetics 199: 379-398 
Kuromori T, Sugimoto E, Shinozaki K (2011) Arabidopsis mutants of AtABCG22, an ABC transporter gene, increase water transpiration and drought susceptibility. Plant J 67: 885-894

Law JA, Ausin I, Johnson LM, Vashisht AA, Zhu JK, Wohlschlegel JA, Jacobsen SE (2010) A protein complex required for polymerase $V$ transcripts and RNAdirected DNA methylation in Arabidopsis. Curr Biol 20: 951-956

Li P, Tao Z, Dean C (2015) Phenotypic evolution through variation in splicing of the noncoding RNA COOLAIR. Genes Dev 29: 696-701

Li Y, Huang Y, Bergelson J, Nordborg M, Borevitz JO (2010) Association mapping of local climate-sensitive quantitative trait loci in Arabidopsis thaliana. Proc Natl Acad Sci U S A 107: 21199-21204

Liu WX, Zhang FC, Zhang WZ, Song LF, Wu WH, Chen YF (2013) Arabidopsis Di19 functions as a transcription factor and modulates PR1, PR2, and PR5 expression in response to drought stress. Mol Plant 6: 1487-1502

Liu Y, Ahn JE, Datta S, Salzman RA, Moon J, Huyghues-Despointes B, Pittendrigh B, Murdock LL, Koiwa H, Zhu-Salzman K (2005) Arabidopsis vegetative storage protein is an anti-insect acid phosphatase. Plant Physiol 139: 1545-1556

Livak KJ, Schmittgen TD (2001) Analysis of relative gene expression data using real-time quantitative PCR and the 2(-Delta Delta C $(T)$ ) Method. Methods 25: 402-408

Mammarella ND, Cheng Z, Fu ZQ, Daudi A, Bolwell GP, Dong $X$, Ausubel FM (2014) Apoplastic peroxidases are required for salicylic acid-mediated defense against Pseudomonas syringae. Phytochemistry

Mauch-Mani B, Mauch F (2005) The role of abscisic acid in plant-pathogen interactions. Current Opinion in Plant Biology 8: 409-414

Meijon M, Satbhai SB, Tsuchimatsu T, Busch W (2014) Genome-wide association study using cellular traits identifies a new regulator of root development in Arabidopsis. 46: 77-81

Milla MA, Townsend J, Chang IF, Cushman JC (2006) The Arabidopsis AtDi19 gene family encodes a novel type of Cys2/His2 zinc-finger protein implicated in ABAindependent dehydration, high-salinity stress and light signaling pathways. Plant Mol Biol 61: $13-30$

Narsai R, Wang C, Chen J, Wu J, Shou H, Whelan J (2013) Antagonistic, overlapping and distinct responses to biotic stress in rice (Oryza sativa) and interactions with abiotic stress. BMC Genomics 14: 93

Nieto-Sotelo J, Ichida A, Quail PH (1994) PF1: an A-T hook-containing DNA binding protein from rice that interacts with a functionally defined $d(A T)$-rich element in the oat phytochrome A3 gene promoter. The Plant Cell 6: 287-301

O'Brien JA, Daudi A, Finch P, Butt VS, Whitelegge JP, Souda P, Ausubel FM, Bolwell GP (2012) A peroxidase-dependent apoplastic oxidative burst in cultured Arabidopsis cells functions in MAMP-elicited defense. Plant Physiol 158: 2013-2027

Ogura T, Busch W (2015) From phenotypes to causal sequences: using genome wide association studies to dissect the sequence basis for variation of plant development. Curr Opin Plant Biol 23: 98-108

Onate-Sanchez L, Vicente-Carbajosa J (2008) DNA-free RNA isolation protocols for Arabidopsis thaliana, including seeds and siliques. BMC Res Notes 1: 93

Pareek A, Sopory SK, Bohnert H (2010) Abiotic Stress Adaptation in Plants: Physiological, Molecular and Genomic Foundation. Springer Netherlands

Pieterse CM, Leon-Reyes A, Van der Ent S, Van Wees SC (2009) Networking by small-molecule hormones in plant immunity. Nat Chem Biol 5: 308-316

Pieterse CM, Van der Does D, Zamioudis C, Leon-Reyes A, Van Wees SC (2012) Hormonal modulation of plant immunity. Annu Rev Cell Dev Biol 28: 489-521

Prasch CM, Sonnewald U (2013) Simultaneous application of heat, drought, and virus to Arabidopsis plants reveals significant shifts in signaling networks. Plant Physiol 162: 1849-1866

Prasch CM, Sonnewald U (2015) Signaling events in plants: Stress factors in combination change the picture. Environmental and Experimental Botany 114: 4-14 
Ramegowda V, Senthil-Kumar M (2015) The interactive effects of simultaneous biotic and abiotic stresses on plants: mechanistic understanding from drought and pathogen combination. J Plant Physiol 176: 47-54

Rasmussen S, Barah P, Suarez-Rodriguez MC, Bressendorff S, Friis P, Costantino P, Bones AM, Nielsen HB, Mundy J (2013) Transcriptome Responses to Combinations of Stresses in Arabidopsis. Plant Physiology 161: 1783-1794

Rejeb IB, Pastor V, Mauch-Mani B (2014) Plant responses to simultaneous biotic and abiotic stress: molecular mechanisms. Plants 3: 458-475

Rodriguez Milla MA, Uno Y, Chang I-F, Townsend J, Maher EA, Quilici D, Cushman JC (2006) A novel yeast two-hybrid approach to identify CDPK substrates: Characterization of the interaction between AtCPK11 and AtDi19, a nuclear zinc finger protein1. FEBS Letters 580: 904-911

Sewelam N, Oshima Y, Mitsuda N, Ohme-Takagi M (2014) A step towards understanding plant responses to multiple environmental stresses: a genome-wide study. Plant Cell Environ 37: 2024-2035

Shaik R, Ramakrishna W (2013) Genes and co-expression modules common to drought and bacterial stress responses in Arabidopsis and rice. PLoS One 8: e77261

Shinozaki K, Yamaguchi-Shinozaki K (2007) Gene networks involved in drought stress response and tolerance. J Exp Bot 58: 221-227

Sokal RR RF (1981) Biometry the Principles and Practice of Statistics in Biological Research (2nd ed.). . WH Freeman and Company, San Francisco, USA

Staats M, Van Kan JAL (2012) Genome update of Botrytis cinerea strains B05.10 and T4. Eukaryotic Cell 11: 1413-1414

Sultana N, Florance HV, Johns A, Smirnoff N (2015) Ascorbate deficiency influences the leaf cell wall glycoproteome in Arabidopsis thaliana. Plant Cell Environ 38: 375-384

Sun G, Zhu C, Kramer MH, Yang SS, Song W, Piepho HP, Yu J (2010) Variation explained in mixed-model association mapping. 105: 333-340

Todesco M, Balasubramanian S, Hu TT, Traw MB, Horton M, Epple P, Kuhns C, Sureshkumar S, Schwartz C, Lanz C, Laitinen RAE, Huang Y, Chory J, Lipka V, Borevitz JO, Dangl JL, Bergelson J, Nordborg M, Weigel D (2010) Natural allelic variation underlying a major fitness trade-off in Arabidopsis thaliana. 465: 632-636

Valerio L, De Meyer M, Penel C, Dunand C (2004) Expression analysis of the Arabidopsis peroxidase multigenic family. Phytochemistry 65: 1331-1342

Van Poecke RMP, Sato M, Lenarz-Wyatt L, Weisberg S, Katagiri F (2007) Natural Variation in RPS2-Mediated Resistance among Arabidopsis Accessions: Correlation between Gene Expression Profiles and Phenotypic Responses. The Plant Cell 19: 40464060

van Rooijen R, Aarts MG, Harbinson J (2015) Natural genetic variation for acclimation of photosynthetic light use efficiency to growth irradiance in Arabidopsis. Plant Physiol 167: 1412-1429

Van Wees SCM, Van Pelt JA, Bakker PAHM, Pieterse CMJ (2013) Bioassays for assessing jasmonate-dependent defenses triggered by pathogens, herbivorous Insects, or beneficial rhizobacteria. Methods in Molecular Biology 1011: 35-49

Wiley

Yoshioka K, Shinozaki K (2009) Signal Crosstalk in Plant Stress Responses.

Yu A, Lepere G, Jay F, Wang J, Bapaume L, Wang Y, Abraham AL, Penterman J, Fischer RL, Voinnet O, Navarro L (2013) Dynamics and biological relevance of DNA demethylation in Arabidopsis antibacterial defense. Proc Natl Acad Sci U S A 110: 2389-2394

Zhou W, Cheng Y, Yap A, Chateigner-Boutin AL, Delannoy E, Hammani K, Small I, Huang J (2009) The Arabidopsis gene YS1 encoding a DYW protein is required for editing of rpoB transcripts and the rapid development of chloroplasts during early growth. Plant J 58: 82-96 


\section{Supporting Information}

Additional supporting information may be download via link

https://www.dropbox.com/sh/hb95t5dwvxbsc1u/AAB2Z7Q1DPSwCCUoaTBBi2Pa?dl=0 


\section{$\mathbb{C} \| \Delta \mathbb{P} \mathbb{\|} \mathbb{E} \mathbb{R} 4$}

\section{Genome wide association mapping of the Arabidopsis thaliana growth response to the sequential combination of drought and Botrytis cinerea infection}

Pingping Huang ${ }^{1}$, Mohamed El-Soda ${ }^{2}$, Katarzyna W. Wolinska ${ }^{1}$, Silvia Coolen ${ }^{4}$, Hans van Pelt ${ }^{4}$, Corné Pieterse ${ }^{4}$, Jan van Kan ${ }^{5}$, Mark G. M. Aarts ${ }^{1}$.

${ }^{1}$ Laboratory of Genetics, Wageningen University, Droevendaalsesteeg 1, 6708 PB, Wageningen, the Netherlands.

2 Department of Genetics, Faculty of Agriculture, Cairo University, Giza, 12613, Egypt.

${ }^{3}$ Plant-Microbe Interactions, Department of Biology, Utrecht University, P.O. Box 800.56, 3508 TB, Utrecht, the Netherlands.

${ }^{4}$ Laboratory of Phytopathology, Wageningen University, Droevendaalsesteeg 1, 6708 $P B$, Wageningen, the Netherlands. 


\section{Abstract}

There is an increasing demand to understand the genetics underlying plant response to multiple stresses. Therefore, we designed this work to study the effect of drought pre-treatment on plant response to a subsequent Botrytis cinerea infection to identify genes responsible for tolerating this combinatorial stress. Genome-wide association mapping was performed on plant rosette dry weight of 350 Arabidopsis thaliana accessions subjected to the combinatorial stress. In addition, Arabidopsis accessions were exposed to a single Botrytis cinerea treatment without any pre-treatment. We identified the effect of a number of significant SNPs that were associated with the DW plasticity under stresses, as well as candidate genes that could play a role in the response to the combinatorial stress. Furthermore, we validated the biological functions of six candidate genes under single drought or Botrytis stress and the combinatorial stress using T-DNA insertion mutants and the transcriptome data of the most extreme eight accessions. Allelic effect was observed on MYB46 gene expression under combinatorial stress comparing to single Botrytis treatment. The role of MYB46, PAL4 and HSFA1b in response to the interaction of drought and Botrytis infection was highlighted. We observed antagonistic interaction between drought and Botrytis infection, and the effect of drought pre-treatment on subsequent Botrytis infection is negative.

\section{Keywords}

Arabidopsis thaliana, genome-wide association mapping, (a)biotic stress tolerance, multiple stresses. 


\section{Introduction}

In nature, plants are exposed to various stress that often occur simultaneously or sequentially. When stress factors interfere negatively with plants, this will limit its performance causing a substantial yield reduction (Hirt and Shinozaki, 2004).

Plant responses to biotic and abiotic stresses are often mediated through hormone-signaling pathways. For example, abscisic acid (ABA) plays a major role in the response to abiotic stress such as drought and salinity (Shinozaki and Yamaguchi-Shinozaki, 2007). Jasmonic acid (JA) and ethylene (ET) signaling pathways are essential in reacting to necrotrophic fungi and chewing insects (Vos et al., 2013 ). Salicylic acid (SA) activates plant defense mechanisms upon the infection with biotrophic fungi (Dodds and Rathjen, 2010; Pieterse et al., 2009). Several additional hormones such as brassinosteroids (BR), auxins (IAA) and cytokinins (CK) are also involved in regulating plant responses to biotic and abiotic stress (Hirt, 2009; Pareek et al., 2010; Pieterse et al., 2012). The interaction between plant response to biotic and abiotic stress can be either synergistic, e.g. positive interactions between ABA and IAA or antagonistic, e.g. negative interactions between $J A$ and $S A, A B A$ and $S A, A B A$ and $J A$, and between ABA and ET (Yoshioka and Shinozaki, 2009).

The mechanisms by which plants respond to individual biotic or abiotic stress were extensively studied at both molecular and cellular levels. For example, recent comparative transcriptome studies identified unique as well as overlapping genes playing a role in Arabidopsis thaliana response to several single biotic and abiotic stress (Narsai et al., 2013; Ramegowda and SenthilKumar, 2015; Sewelam et al., 2014; Shaik and Ramakrishna, 2013). In contrast, far less information is known on the plant response to simultaneous or consecutive biotic and/or abiotic stresses. Generally, consecutive stresses trigger the expression of specific multiple stress-responsive genes, indicating a tailored combinatorial stress signaling network (Atkinson and Urwin, 2012; Sewelam et al., 2014). For example, earlier studies (Anderson et al., 2004; Pré et al., 2008; Vos et al., 2013; Yoshioka and Shinozaki, 2009) showed that Botrytis infection triggers the expression of defense responsive genes such as 
PDF1.2 in the JA-ET signaling pathway through the ERF branch, which interacts negatively with the drought induced $A B A$ responses.

The present study focused on identifying genes underlying Arabidopsis thaliana responses to consecutive drought and infection by a necrotrophic fungus, Botrytis cinerea. The objectives were to: 1) observe the effect of the prior drought stress on subsequent Botrytis infection, 2) study the variation in allelic effect in response to single Botrytis and the combinatorial stresses and 3 ) identify genes that could play a role in the response to the single and combinatorial stresses. To achieve these goals we conducted genome-wide association mapping (GWA-mapping) for the rosette dry weight (DW) of 350 Arabidopsis accessions (Baxter et al., 2010; Li et al., 2010). Our results identified a number of genes exhibited allelic effects on combinatorial stress responses, and reported the new role of several known gene in response to the consecutive drought and Botrytis infection. 


\section{Materials and Methods}

\section{Plant material and growth conditions}

For GWA-mapping experiments, the HapMap population (Li et al., 2010) was used. Seeds of the T-DNA insertion mutants (Supplementary Table 1) were ordered from The Nottingham Arabidopsis Stock Centre (NASC) (http://Arabidopsis.info/). Seeds of the HEAT SHOCK TRANCRIPTION FACTOR $1 \mathrm{~b}$ (HSFA1b) over-expression lines and its wild-type are described by Bechtold et al (2013).

Seeds were stratified at $4^{\circ} \mathrm{C}$ for two days in the dark, then sown on river sand and watered with half-strength Hoagland nutrient solution as described by Van Wees et al (2013). Plants were grown in a climate-controlled growth chamber under short day conditions ( $8 \mathrm{hrs}$ day/16 hrs night) at $21^{\circ} \mathrm{C}, 70 \%$ relative humidity and $100 \mu \mathrm{mol} \mathrm{m} \mathrm{m}^{-2} \mathrm{~s}^{-1}$ irradiation. Fourteen-day-old seedlings were transferred to individual pots filled with a mixture of river sand and peat $(1: 1, v / v)$ and watered three times per week, at the bottom of each pot.

\section{Botrytis spore preparation}

Botrytis cinerea strain B05.10 (Staats and Van Kan, 2012) was grown for two weeks at room temperature on half-strength potato dextrose agar plates (PDA; Difco Laboratories) containing $100 \mu \mathrm{g} / \mathrm{ml}$ penicillin and $200 \mu \mathrm{g} / \mathrm{ml}$ streptomycin. Spores were collected, filtered through glass wool and then resuspended in half-strength potato dextrose broth (PDB; Difco Laboratories) to a final density of $1 \times 10^{5}$ spores $/ \mathrm{ml}$. Three hours after incubation spores were used for inoculation by applying six $5-\mu l$ droplets on six Arabidopsis leaves.

\section{Single and combinatorial stress application}

Screening the Hapmap population: For the combinatorial stress experiments, i.e. drought on subsequent Botrytis, four-week-old Arabidopsis plants of the Hapmap population were subjected to drought by withholding water for seven days and then re-watered to recover for one day. Thereafter, plants were inoculated with Botrytis by pipetting six $5-\mu l$ droplets of spore suspension $\left(1 \times 10^{5} \mathrm{spores} / \mathrm{ml}\right)$ onto six leaves (one droplet per leaf). For the single Botrytis 
treatment, 5-week-old plants were inoculated with Botrytis as described above. Plants in both treatments were incubated at $100 \%$ relative humidity $(\mathrm{RH})$ for 3 days, after which samples were collected (Figure 1.a).

Screening T-DNA mutants: In addition to the two treatments described in the previous paragraph, two mock treatments replaced the Botrytis treatment where six 5- $\mu$ l droplets PDB were applied on six leaves per plants. Two additional treatments were applied in which one treatment involved watering plants for 34 days and the other treatment involved watering plants for 27 days and then subject them to drought for six days (Figure 1.b). After mock or Botrytis infection, plants were maintained at $100 \% \mathrm{RH}$ for three days. For drought treatments, six plants of each mutant/wild-type were used. For the Botrytis treatment, 48 leaves (8 plants) of each mutant/wild-type were used. Samples for gene expression were collected twice, directly after drought stress and three days after Botrytis treatment.

Screening extreme accessions of the Hapmap population: This experiment was carried out with the same design as described in the previous paragraph except that an additional sample for gene expression was collected after one day (at time point 2, Figure 1.b) of Botrytis infection treatment. 
a.

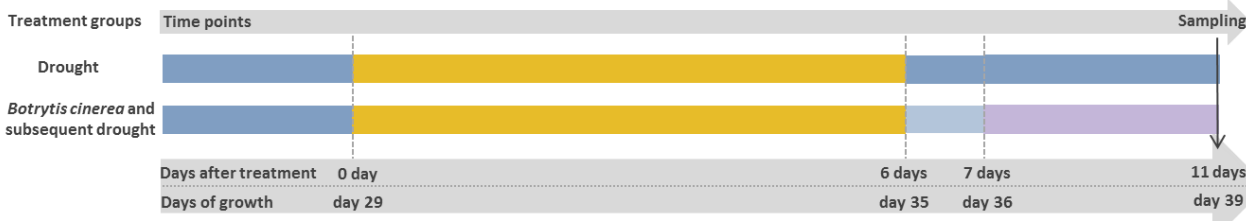

b.

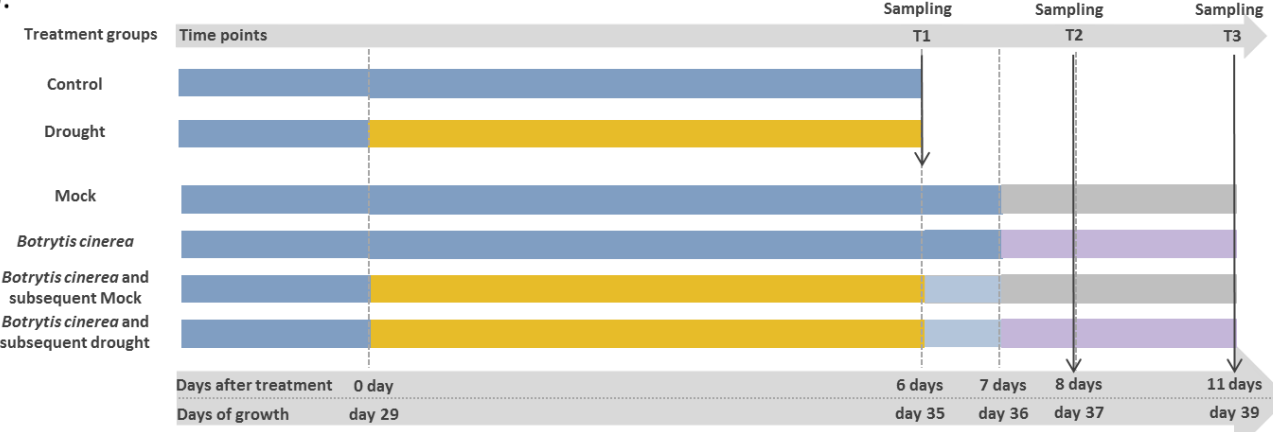

Figure 1. Experimental design for treatment of Arabidopsis plants subjected to drought, Botrytis cinerea infection and combinatorial stress. Conditions are indicated in different colors: control (dark blue); drought (yellow); Botrytis treatment (purple); rehydration after drought (light blue). Dashed line indicate days after treatment and the growth date. Vertical arrows indicate sampling time point (a.) and sampling time points (T1-T3). a. experimental design for screening Hapmap accessions in response to drought and the consecutive drought and Botrytis infection, b. experimental design for T-DNA insertion mutants, wild-type and Arabidopsis accessions in different conditions.

\section{Genome-Wide Associations mapping}

GWA-mapping was performed using the scanGLS software as described by Kang et al (2010) and Kruijer et al (2015). In brief, this approach performs generalized least squares (GLS) calculations conditional on variance components estimated in the model without markers. Thereafter, SNPs with minor allele frequency (MAF) lower than 5\% were excluded. Marker-trait associated $P$-values at $10^{-4}$ with type I error was used to evaluate significant SNPs. The proportion of explained phenotypic variance of each significant SNP was the criterion used to identify significant SNPS as described by Sun et al (2010). The information of SNPs in the linkage disequibrium (LD) with the significant SNPs were identified by the online LD tool (http://dev3.ab.wur.nl/AthaLD), as well as the information of coding sequence substitution and amino acid substitution. 


\section{Selection of candidate genes}

Gene ontology annotation of selected candidate genes for the quantitative trait loci (QTL) identified after the GWA analysis was found in The Arabidopsis Information Resource (TAIR; www.Arabidopsis.org/tools/bulk/go/index.jsp). Genes characterized by one or more of the following criteria were considered as candidates: 1) Genes known to be responsive to biotic and abiotic stimulus and expressed under abiotic stress and hormonal (JA and ABA) treatments; 2) Genes with reported physiological functions in response to stress conditions and expressed under abiotic stresses and hormonal (JA and $A B A$ ) treatments (http://bar.utoronto.ca/efp/cgi-bin/efpWeb.cgi). 3) Genes with reported physiological functions in response to stress conditions and responsive to biotic and abiotic stimuli.

\section{Quantitative reverse transcriptase ( $q R T$ )-PCR}

To determine the expression of the candidate genes in Arabidopsis accessions with an extreme phenotype compared to the whole set of accessions, total RNA was extracted from rosettes following the protocol described by Onate-Sanchez and Vicente-Carbajosa (2008). Thereafter, cDNA was synthesized from $800 \mathrm{ng}$ of total RNA using an iScript cDNA synthesis kit (BioRad) at $25^{\circ} \mathrm{C}$ for 5 minutes, $42^{\circ} \mathrm{C}$ for 30 minutes and $85^{\circ} \mathrm{C}$ for 5 minutes. cDNA was diluted ten times to be used in the quantitative reverse transcription PCR (qRT-PCR). Based on the transcriptome data collected from several drought, osmotic and Botrytis studies using the Genevestigator web tool http://refgenes.org/rg, unnamed gene At3G19460 was considered to be the most stable gene expressed across experiments and was selected as reference gene for gene expression studies. The qRT-PCR was performed using iQ SYBR ${ }^{\circledR}$ Green Supermix (cat. no. 170-8885) on a Bio-Rad CFX96 real-time PCR system set at $95^{\circ} \mathrm{C}$ for 4 minutes, followed by 40 cycles of $95^{\circ} \mathrm{C}$ for 10 seconds and $55^{\circ} \mathrm{C}$ for 30 seconds. The primer sequences used to amplify the four investigated genes, i.e. TUBLINE 5 (TUB5), PHENYLALANINE AMMONIA-LYASE 4 (PAL4), MYB DOMAIN PROTEIN 46 (MYB46) and HEAT SHOCK FACTOR 1b (HSFA1b), are provided in Supplementary Table 2 and their expression was measured in eight accessions, i.e. Est-1 (CS76127), Sei-0 (CS28729), Kulturen-1 (CS76156), RRS7 (CS28713), (Col-0 (CS76113), Rsch-4 (CS76222), Cnt-1 (CS28160), Can-0 
(CS76109). Gene primers were designed at the identical sequence region of the eight accessions.

\section{Statistical analysis}

Pearson's correlation coefficient, one-way and 2-way ANOVAs were performed using GenStat for Windows $16^{\text {th }}$ Edition (VSN International Ltd., Hempstead, UK). Three-way ANOVA was performed as described by (Sokal RR, 1981). Student's t-test was used to compare rosette dry weight (DW) and lesion diameter of plants grown under the combinatorial stress with those grown under the single Botrytis stress. For lesion diameter, 48 leaves (eight plants) per accession or line were used, whereas, for DW, five plants per accession or line were used. 


\section{Results}

\section{Phenotyping the Arabidopsis accessions under drought and the combinatorial stress}

To identify genes that are involved in the response to consecutive abiotic and biotic stress the Arabidopsis HapMap population was grown for four weeks under normal, well-watered conditions and then subjected to one week of drought. Thereafter plants were watered and allowed to recover for one day (rehydration), before being infected with Botrytis. Rosettes of plants were harvested after three days Botrytis treatment and used to determine rosette dry weights (DW) (Figure 1.a). High broad sense Heritabilities $\left(\mathrm{H}^{2}\right)$ of DWs under single Botrytis treatment (0.69) and the combinatorial stress $(0.73)$ were observed. High Pearson's correlation coefficient $(0.81)$ between the two treatments was found. Two-way ANOVA of DW was used to test the interaction between treatments and accessions. Significant interaction between treatments and accessions was found $(p<0.001)$, indicating that there is genetic variation for the drought pre-treatment effect.

\section{Univariate GWA-mapping of DW residuals identified SNPS associated with phenotypic plasticity}

GWA-mapping using the DW residuals that was calculated from a regression analysis of DW under consecutive drought and Botrytis to DW under single Botrytis treatment, and revealed 34 significantly associated SNPs (Figure 2 and Table 1) that are located in or very close to 30 genes. 


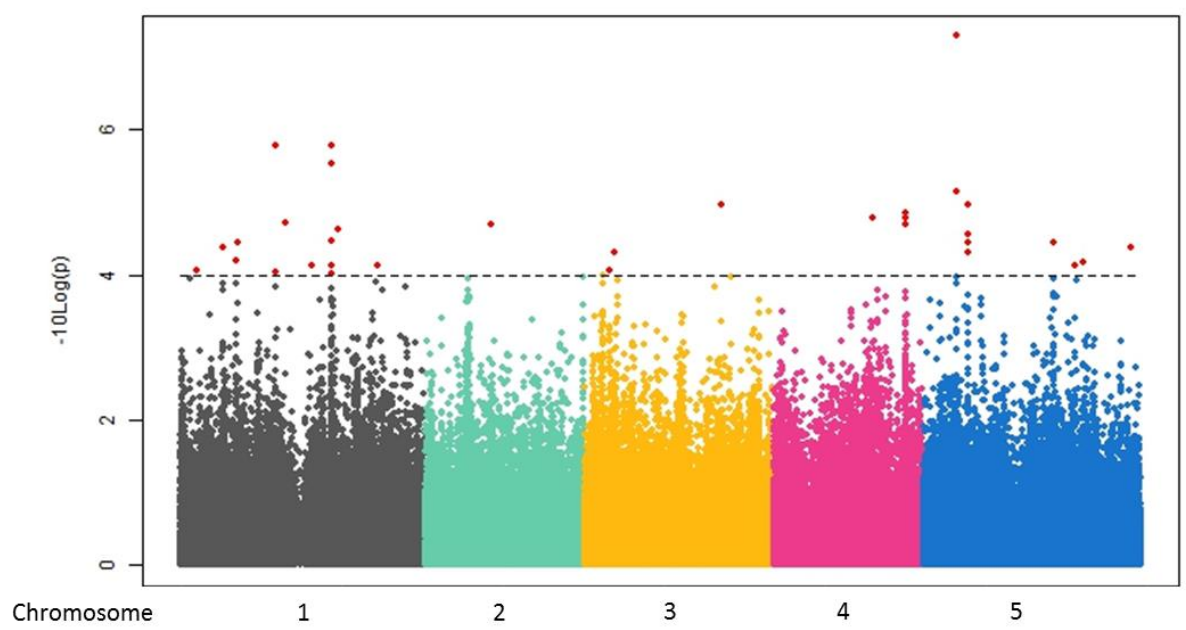

Figure 2. univariate GWA-mapping on DW for consecutive drought and Botrytis stress responses identified 34 significant SNPs $(-\log (p)>4)$ (red) that located on five chromosomes (black, green, yellow, red, blue).

SNP m168340 exhibited strong association with $-\log (p)$ score at 7.29 (Figure 2 and Table 1). The non-Col like allele of SNP m168340 presented strong effect on combinatorial stress responses and explained $8.06 \%$ genetic variation of accessions in response to the combinatorial stress. This SNP is not located or very close to any gene, but when looking at the Linkage Disequilibrium (LD) of the SNP, a large locus (150kb) including 74 SNPs that are associated to 16 genes was found. Another four SNPs exhibited higher $-\log (p)$ score $(>5)$ than the other SNPs (Table 1). SNP m168339 is in LD with m16340 and is present at the same locus. SNP $\mathrm{m} 20853$ is located at the $2.2 \mathrm{~kb}$ upstream region of MULTIPLE ORGANELLAR RNA EDITING FACTOR 5 (MOREF5; AT1G32580), which acts as a cofactor of pentatricopeptide repeat proteins (PPR proteins) for RNA editing in plastids and mitochondria in flowering plants (Zehrmann et al., 2015). The synonymous non-Col like allele of the SNP explained $5.82 \%$ phenotypic variance. Two SNPs $\mathrm{m} 370070$ and m370071 are located at the exon of an unnamed gene AT1G50650 with unknown molecular function. The non-Col like alleles of the two SNPs explained respectively $5.78 \%$ and $6.03 \%$ phenotypic variations of accessions under the combinatorial stress. The synonymous substitution of SNP $\mathrm{m} 370070$ is from G (Col-0 like) to T (non-Col like). The nonsynonymous substitution of SNP m370071 is from A (Col-0 like) to G (non- 
Col like) and causes an isoleucine to threonine change, but the on effect on combinatorial stress responses was found when comparing the non-Col allele to Col-0 allele.

SNPs such as m157533, m157535, and m151030 exhibited large allelic effects on combinatorial stress responses among the 34 significant SNPs and displayed high $-\log (p)$ score ( 4.8; Table 1$)$. SNPs m157533 and m157535 are associated with unnamed gene AT4G34400; and another SNP m157515 (4.69) was found in LD with $\mathrm{m} 15735$ and is associated with gene ARIADNE 1 (ARI1; AT4G34370), which could interact with the ubiquitin-conjugating (E2) enzymes for ubiquitination (Mladek et al., 2003). The coding sequence substitution of SNPs m157533 and m151030 were synonymous, while SNP m157535 in ARI1 displayed a nonsynonymous substitution from G (Col-0 like) to A (non-Col like) leading to an amino acid substitution from aspartic acid to asparagine. We found that the amino acid substitution in $A R I 1$ has a negative effect on combinatorial stress responses as reduced DW ratio was observed under the combinatorial stress when compared to Botrytis infection (Figure 3.a). The SNP m151030 present in the 5th exon of the 3XHIGH MOBILITY GROUP-BOX2 (3XHMG-BOX2; AT4G23800) gene showed a synonymous substitution and is unlikely to cause an effect on the protein function. However, another SNP is tightly linked (LD $=0.98$ ) to SNP m151030 and displayed a nonsynonymous substitution from $\mathrm{G}$ to $\mathrm{C}$ resulting in a serine to threonine change. The non-Col allele of the SNP exhibited negative effects on combinatorial stress tolerance in comparison to the Col-0 like allele (Figure 3.b) Gene PROTEIN ARGININE METHYLTRANSFERASE 4A (PRMT4A; AT5G49020; Table 1) encodes a type I protein arginine methyltransferase which plays a role in regulating flowering time through the FLOWERING LOCUS C (FLC)-dependent pathway (Niu et al., 2008). The SNP m201187 present in PRMT4A displayed a $-\log (p)$ score of 4.12 and was a sequence substitution from $\mathrm{G}$ to $\mathrm{T}$ causing a valine to phenylalanine substitution. Plants with this genotype can cope less with combined stress when compared with plants that contain the Col-0 like allele (Figure 3.c). 
a.

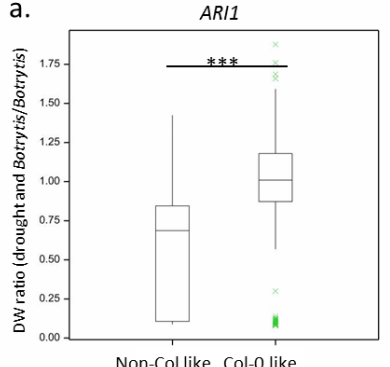

b.

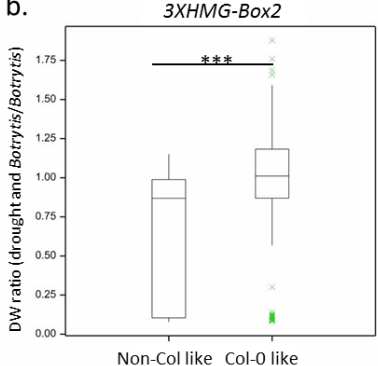

c.

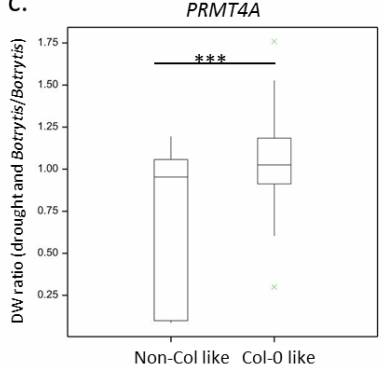

Figure 3. Boxplots of the combination of two types allele (Col-0 like and non-Col like) of SNPs associated with gene ARI1 (a), 3XHMG-Box2 (b) and PRMT4A (c). Student's t-test was used to compare the differences of the Col-0 like and non-Col like alleles of each gene. $* * *$ indicates significant differences $(p<0.0001)$ 


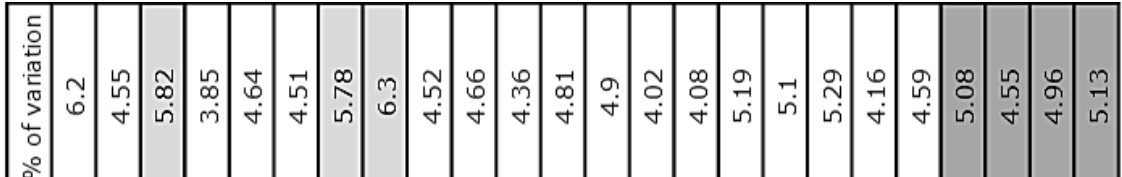

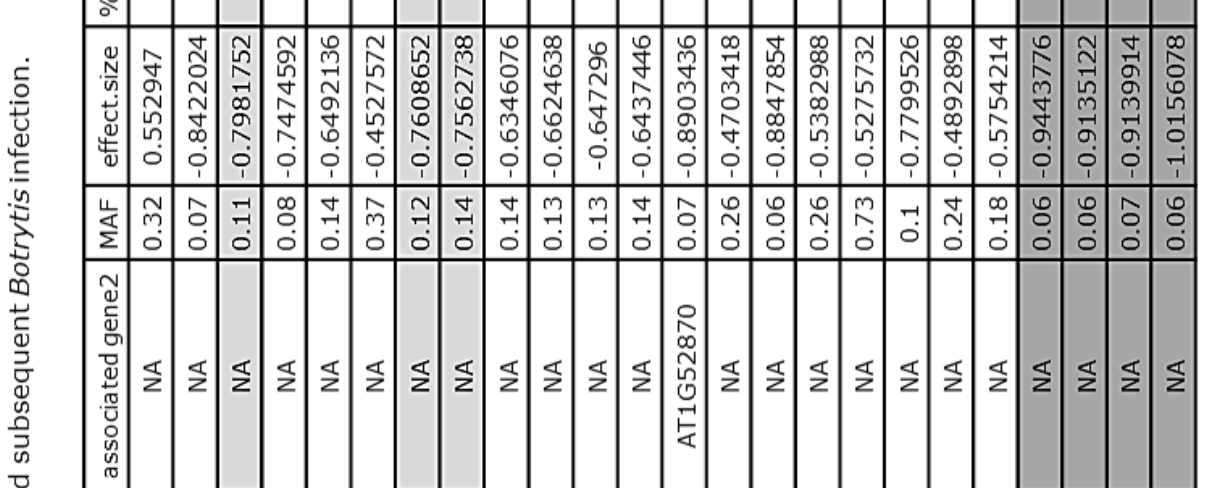

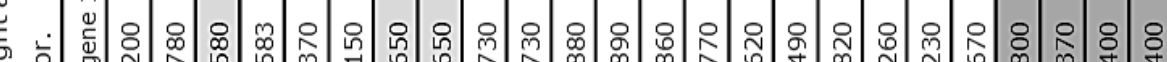

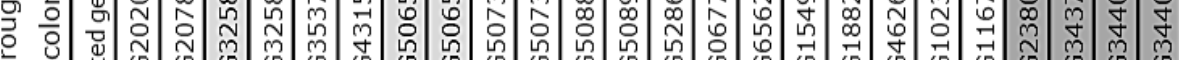
눈

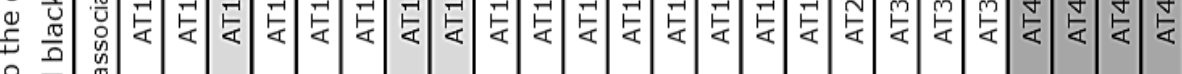
잉

¿

힌

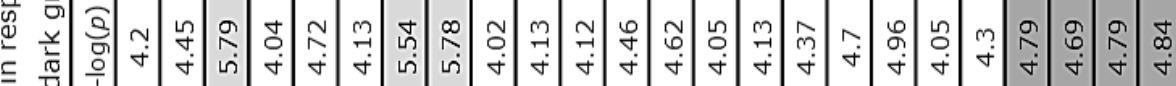

$\sum_{\substack{n \\ 0}}^{n}$

†.

ลิ ซ্巳

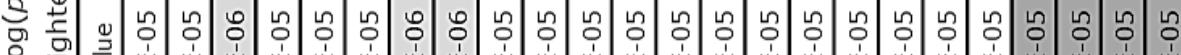

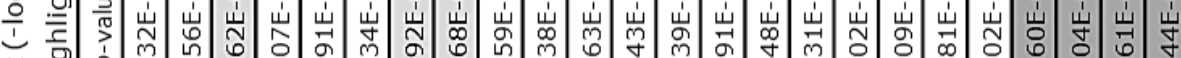

䒕

응

茫

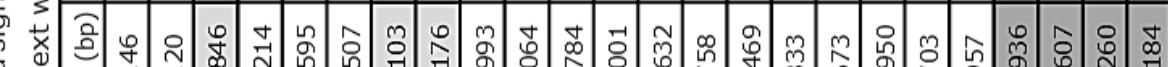

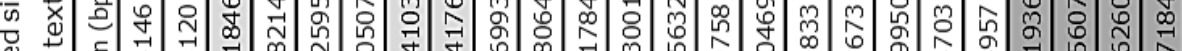

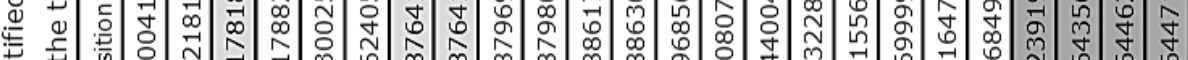

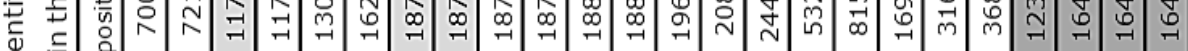
-

요

은

흔

है

$\sum$

$\frac{\Phi}{\frac{1}{4}} \frac{\mathrm{o}}{\mathrm{c}}$

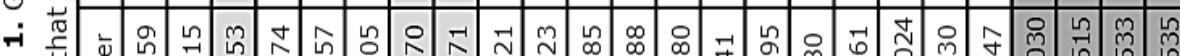

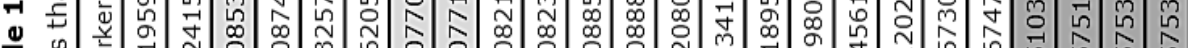

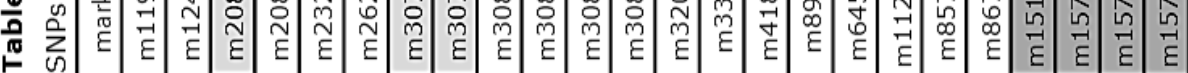




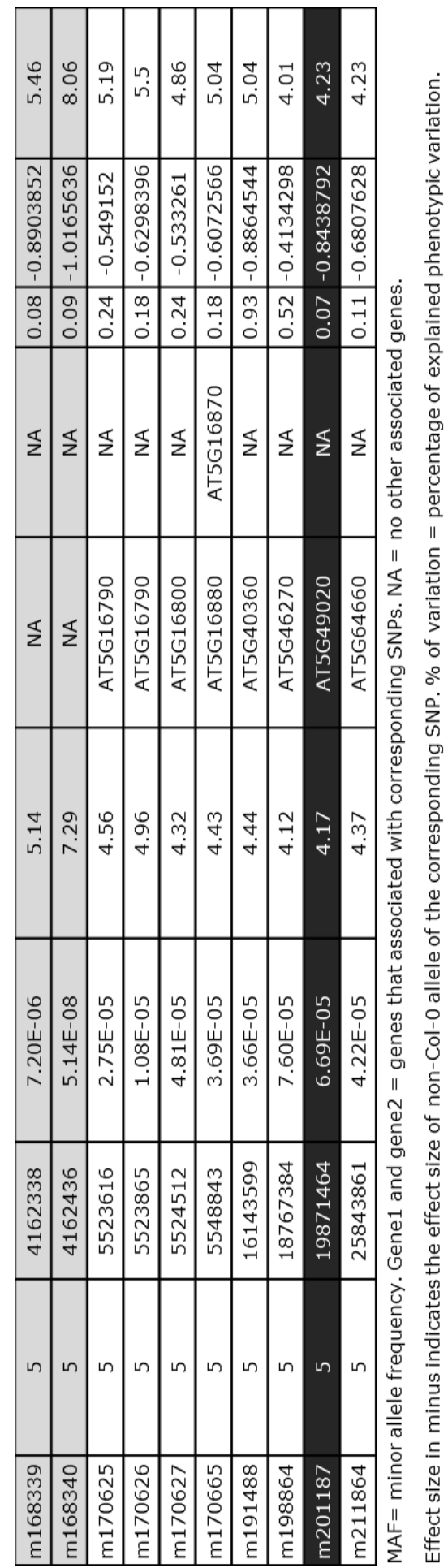




\section{Genes play a role in response to combinatorial stress}

Another 191 candidate genes were in linkage disequilibrium (LD >0.3) with the associated significant SNPs, increasing the total number to 221 candidate genes that could explain the allelic variance revealed by challenging the population with the single and combinatorial stresses (Supplementary Table 3). Some candidate genes were reported to play a role in response to biotic, abiotic or plant hormone treatments, but none were reported a role in response to both drought and Botrytis infection. To study the interaction of drought and Botrytis infection, six candidate genes: DREB2A-INTERACTING PROTEIN 1 (DRIP1; AT1G06770), ARMADILLO BTB PROTEIN 1 (ABAP1; AT5G13060), TUBULIN BETA-5 CHAIN (TUB5; AT1G20010), PHENYLALANINE AMMONIA-LYASE 4 (PAL4; AT3G10340), MYB DOMAIN PROTEIN 46 (MYB46; AT5G12870) and HEAT SHOCK FACTOR $1 B$ (HSFA1b) (AT5G16820), were selected based on available homozygous T-DNA insertion mutants. drip1, tub5 and pal4 mutants were tolerant to drought and myb46, abap1 and hsfa1b were susceptible to drought when compared to the wild-type (Figure 4.a). Under Botrytis infection, pal4 mutant was less infected when compared to the wild-type (Figure 4.b). Comparing the DW of plants under combinatorial stress to Botrytis infection without pre-treatment, pal4 and hsfa $1 b$ exhibited reduced DW when compared to the wild-type (Figure 4.c). When comparing the lesion diameter under combinatorial stress to Botrytis infection, tub5 and myb46 mutants exhibited less spread lesion diameter than the wild-type (Figure 4.d) and $h s f a 1 b$ displayed larger spread lesion diameter than the wild-type. 
a.

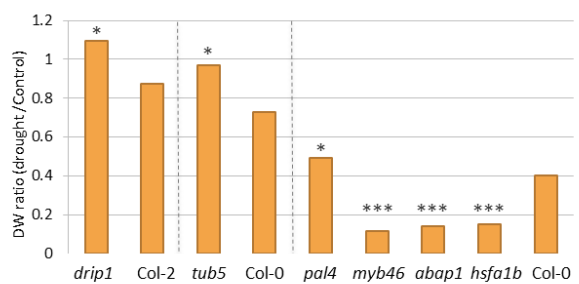

C.

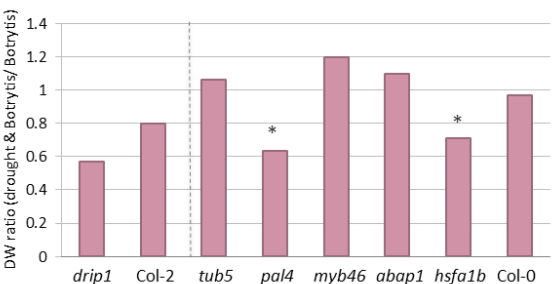

b.

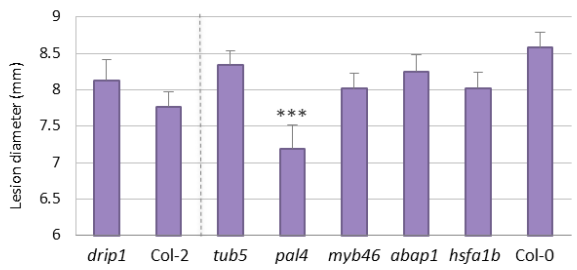

d.

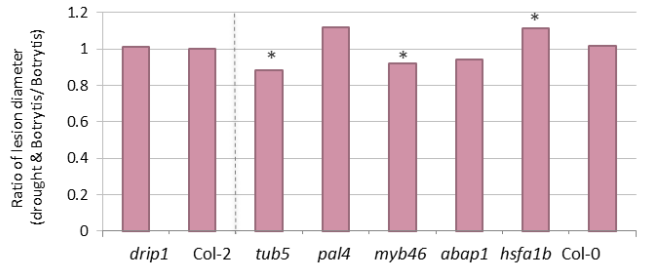

Figure 4. Phenotype of T-DNA insertion mutants of genes DRIP1, TUB4, PAL4, MYB46, $A B A P 1$ and $H S F A 1 B$, in response to drought (yellow, a.), in response to Botrytis infection (purple, b), in response to the combinatorial stress (pink, c \& d). Dashed line indicates Col-2 is the wild-type for drip1 mutant.

a. Ratio of rosette dry weight (DW) of the wild-types (Col-0, Col-2) and the mutant plants in response to drought, when compared to the control.

b. Lesion diameter $(\mathrm{mm})$ of these genotypes under Botrytis treatment.

c. Ratio of DW of these genotypes under combinatorial stress, when compared to Botrytis without pre-treatment.

d. Ratio of lesion diameter of these genotypes under Botrytis treatment.

Two-way ANOVA was used for all figures. Standard error for DW was calculated from at least eight plants, standard error for lesion diameter was calculated from at least 48 leaf replicates (6 leaves per plant $\times 8$ biological repeat). * indicates mutants with a significant difference when compared to Col-0 wild type $(* p<0.05, * * * p<0.001)$

Two overexpression lines HSFA1bOX1 (WS-2 background) and HSFA1bOX2 (Col-0 background) were used to observe the effect on Botrytis and the combinatorial stress responses. Plants that overexpressed HSFA1B were more tolerant to drought than wild-types (Figure 5.a). Under Botrytis infection, reduced lesion diameter was observed from the two overexpression lines when compared to the wild-types (Figure 5.b). We found HSFA1bOX2 is more tolerant to the combinatorial stress when compared to the wild-type (Figure 5.c) and only slightly more tolerance was observed in $H S F A 1 b O X 1$, though not significant. Comparing the lesion diameter under combinatorial stress to single Botrytis infection, the two overexpression lines were more sensitive to Botrytis when pre-treated with drought than the wild-types (Figure 5.d). Less spread lesion 
diameter of the two overexpression lines was found when compared to the wildtypes under combinatorial stress (Figure 5.e).

a.

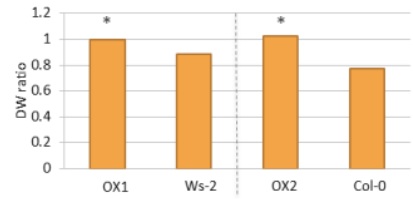

d.

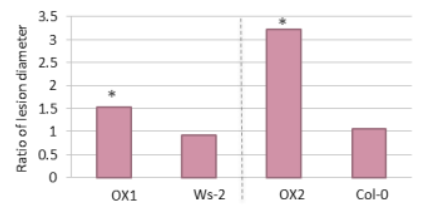

b.

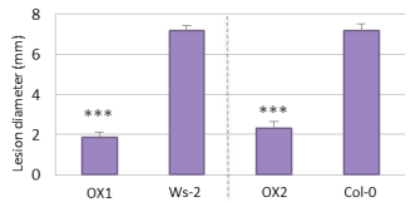

e.

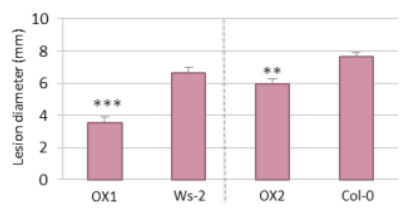

C.

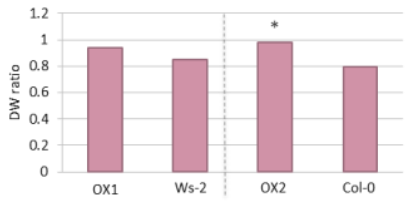

Figure 5. Phenotype of HSFA1b overexpression lines and the wild types in response to drought (yellow, a.), in response to Botrytis infection (purple, b), in response to the combinatorial stress (pink, c, d \& e), Dashed line indicates the HSFA1b overexpression line 1 (OX1) and the wild-type Ws-2; HSFA1b overexpression line 2 (OX2) and the wild-type Col-0.

a. Ratio of rosette dry weight (DW) of the two wild-types (Ws-2, Col-0) and the two overexpression lines (OX1, OX2) in response to drought when compared to the control.

b. Lesion diameter of these genotypes under Botrytis treatment.

c. Ratio of DW of these genotypes under combinatorial stress, when compared to Botrytis without pre-treatment.

d. Ratio of lesion diameter of these genotypes under combinatorial stress, when compared to Botrytis without pre-treatment.

e. Lesion diameter of these genotypes under combinatorial stress.

Two-way ANOVA was used for all figures. Standard error for DW was calculated from at least eight plants, standard error for lesion diameter was calculated from at least 72 leaf replicates ( 6 leaves per plant $\times 12$ plants). * indicates mutants with a significant difference when compared to Col-0 wild type $(* p<0.05, * *<0.01, * * * p<0.001)$.

To observe the effect of drought pre-treatment on gene expression in sequential Botrytis infection condition, the relative expression fold change of genes TUB5, PAL4, MYB46 and HSFA1b were measured in eight Arabidopsis accessions that either were susceptible (Est-1, CS76127; Sei-0, CS28729; Kulturen-1, CS76156; or RRS-7, CS28713) or tolerant (Col-0, CS76113; Rsch-4, CS76222; Cnt-1, CS28160; or Can-0, CS76109) to the combinatorial stress treatment in comparison to Botrytis stress alone (phenotype result was from the screening of the Hapmap accessions in the two conditions). TUB5 was downregulated under drought but up-regulated under Botrytis infection in all accessions (Figure 6.a). We found drought pre-treatment enhance the downregulation of TUB5 in sequential Botrytis infection condition. PAL4 was downregulated under drought but up-regulated under Botrytis treatment (Figure 6.b). 
The gene was up-regulated under combinatorial stress but the expression fold change was reduced in all accessions when compared to the single Botrytis treatment. MYB46 was up-regulated in all conditions, but the expression fold change was reduced in some accessions under combinatorial stress in comparison to the single Botrytis treatment (Figure 6.c). Variations of HSFA1b gene expression among accessions in stress conditions were observed (Figure 6.d). No significant effect of the interaction between drought and Botrytis on HSFA1b expression was observed.
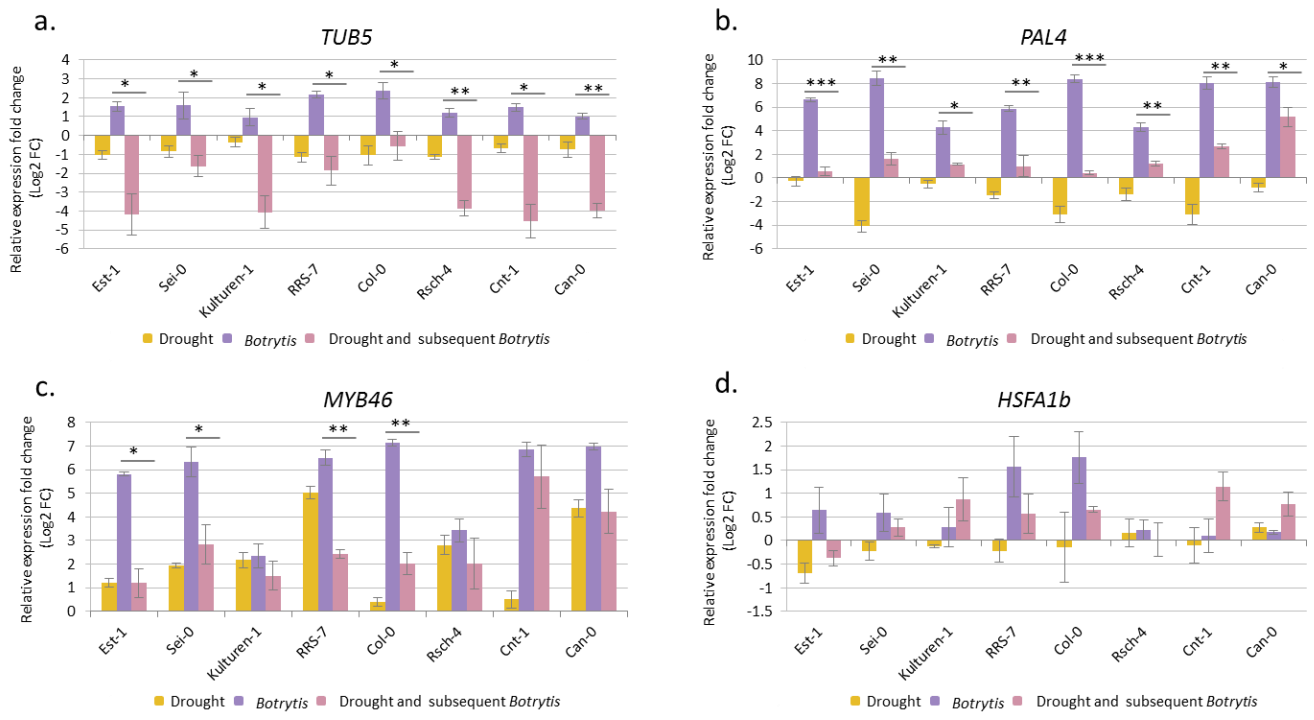

d.

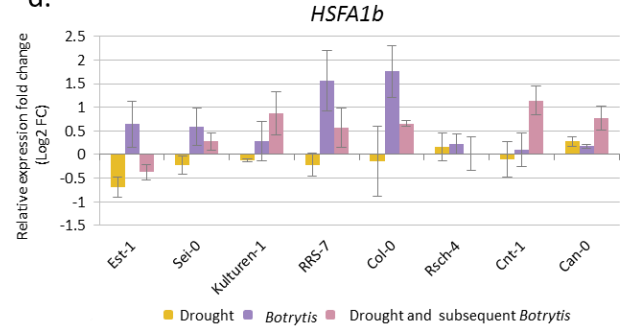

Figure 6. Relative expression fold change at Log2 scale of TUB5 (a.), PAL4 (b.), MYB46 (c.) and HSFA1b (d.) expression in eight Arabidopsis accessions after exposure to drought (yellow), after Botrytis infection (purple) and after exposure to drought and followed by Botrytis infection (pink), compared to control. Log2 scale at 1, 2, 3, 4, 5, 6, 7, 8 = actual expression fold change of $2,4,6,8,16,32,64,128,256$ times. In all figures, two-way ANOVA was used to compare accession under combinatorial stress to single Botrytis stress. Standard error was calculated from 3 plants per accession. * indicates mutants with a significant difference when compared to drought $(* p<0.05, * * p<0.01, * * *<0.001$ ) 


\section{Discussion}

\section{Mapping significant SNPs associated with drought, Botrytis and combinatorial stress}

This study uncovers the genes for which there is natural allelic variation affecting the Arabidopsis response to the single and combinatorial response to drought and Botrytis exposure. GWA-mapping revealed 221 candidate genes that could have allelic effects on Botrytis and the combinatorial stress responses. High heritability was found under both conditions, indicating that the variation of accessions in responses to stresses is due to its genetic variation. However, each identified individual QTLs explained only small genetic variances (Table 1). It is possible that a GWAs analysis on a complex trait, such as DW, could result more QTLs but with less effect size, while a simple trait could result less QTLs but with large effect size (Louthan and Kay, 2011). Several studies combined association mapping with linkage mapping and identified flowering time associated QTLs (Brachi et al., 2010), Hyaloperonospora arabidopsidis ex parasitica (Hpa) resistance genes (Nemri et al., 2010), endoreduplication related genes (Sterken et al., 2012) and shoot regeneration genes (Motte et al., 2014). Thus, to confirm the loci associated with combinatorial stress response follow-up studies of QTL analysis using parental populations will be needed.

We observed nonsynonymous amino acid substitution caused by the non-Col alleles of SNPs on gene ARI1, 3XHMG-Box2 and PRMT4A and observed negative effects of the non-Col like alleles in response to the combinatorial stress when compared to the Col-0 like alleles. This shows that the three genes could be causative genes with allelic effects on the combinatorial stress responses. PRMT4A is involved in the FLOWERING LOCUS C (FLC)-dependent pathway, but has a redundant function with PRMT4B (AT3G06930) because only the prmt4aprmt4b double knockout mutant exhibited enhanced expression of FLC (Niu et al., 2008). The GWAs analysis identified PRMT4A as the candidate gene for combinatorial stress but not PRMT4B, showing a role of PRMT4A to combinational stress response. 
Two SNPs m370070 and m370071 associated to gene AT1G50650 exhibited nonsynonymous amino acid substitution but did not affect the combinatorial stress responses. This shows that the two SNPs itself are not the causal SNPs but other SNPs in LD or the two SNPs in combination with other SNPS in LD could be casual SNPs.

Although we did not validate the allelic effects of candidate genes TUB5, PAL4, MYB46 and HSFA1b on stress responses, the mutants exhibited different phenotype in response to combinatorial stress when compared to the wild-type. This shows that the allelic variation of the four genes could contribute to the genetic variance to combinatorial stress responses. In this study, allelic effects on gene expression was observed for MYB46. The expression of MYB46 was reduced by the drought pre-treatment in most tolerant accessions in sequential Botrytis condition, but not in susceptible accessions (except in Col-0) (Figure 5.c). Other genes such as DRIP1 and SUPERSENSITIVE TO ABA AND DROUGHT 1 (SAD1, At5g48870), which are two candidate gene in LD with the significant SNPS, could show the effect of allele variation in stress responses. DRIP1 can interact with DREB2A (DEHYDRATION-RESPONSIVE ELEMENT BINDING PROTEIN2A) hereby inhibiting DREB2A function by marking the protein for proteasomal degradation via ubiquitination (Qin et al., 2008). Recently, similar negative regulation of DREB2A by DRIP1 was reported in cowpea (Sadhukhan et al., 2014) indicating that this part of the drought response in plants is conserved and likely to be tightly controlled. We showed that loss of DRIP1 resulted in a tolerance to drought stress when compared to wild-type (Figure 3.a). SAD1 is a multifunctional protein involved in mRNA splicing, export and degradation (Xiong et al., 2001). The sad1 mutant is impaired in regulation of the ABA-dependent stress responsive genes (Xiong et al., 2001) and has an early flowering phenotype (Perez-Santangelo et al., 2014), a common drought escape strategy (Franks, 2011; Sherrard and Maherali, 2006). Moreover, the finding of $S A D 1$ shows that there is possibly extensive degradation at the mRNA level (Golisz et al., 2013) and can affect the protein level during stress. Thus, the allele effects of SAD1 on mRNA degradation is an area interesting to explore. To confirm the effects of the allele variations on combinatorial stress responses, complementation tests to validate the biological role of the natural alleles of the described genes are necessary. 


\section{The role of candidate genes in response to drought and Botrytis stresses}

The knockout mutants of candidate genes MYB46, PAL4 and TUB5 exhibited different phenotypes compared to wild type and the genes displayed differential gene expression under the combinatorial stress when compared to the single Botrytis treatment. Antagonistic interaction between drought and Botrytis was for pal4 mutant, and the same effect was found on TUB5 gene expression. Negative effect of drought pre-treatment was observed in hsfa1b, tub5 and myb46 mutants in sequential Botrytis infection. The negative effect of drought pre-treatment was observed on the expression of PAL4 and MYB46 as well.

The MYB46 transcription factor regulates the expression of many secondary wall biosynthesis genes such as cellulose synthases genes (CESA4, CESA7, CESA8) and activates MYB family and NAC family transcription factors, which are involved in lignin and secondary wall biosynthesis (Ko et al., 2014). CESA8 is also a drought and osmotic tolerant gene (Chen et al., 2005). Thus, the susceptible phenotype of myb46 to drought stress could be due to less expression of CESA8. With regard to the Botrytis stress, one study reported that the myb46 mutant is tolerant to Botrytis infection because the Botrytis defense responsive gene PDF1.2 is highly expressed in the mutant and that led to an enhanced tolerance to Botrytis stress (Ramirez et al., 2011). However, in the present study, we did not observe the same phenotype of myb46 mutant in response to Botrytis infection when compared to the wild type. Downstream of MYB46 are MYB58 and MYB63 that are involved in regulating lignin biosynthesis through regulating the expression of several monolignol biosynthesis genes including PAL1 and PAL4 (Kim et al., 2014; Ko et al., 2014; Ko et al., 2009; Zhou et al., 2009). PAL4 is a member of the Phenylalanine ammonia-lyase (PAL) gene family that contains four genes PAL1-PAL4 that respond to various stresses such as pathogens, cold, wounding and UV (Huang et al., 2010). PAL genes are involved in salicylic acid (SA) accumulation, the knockout mutants of PAL genes exhibited $75 \%$ reduction of SA when compared to the wild type (Dempsey et al., 2011). SA can antagonize JA (Yoshioka and Shinozaki, 2009). Thus, we hypothesis that the increased tolerance to Botrytis is due to reduced SA concentration in pal4 mutant, which led to increased JA accumulation. 
Two SNPs were located at the exon of HSFA1b and were in LD (LD>0.46) with significant SNP $\mathrm{m} 170625(-\log (p)=4.56)$. The non-Col like alleles of the two SNPs led to nonsynonymous amino acid substitution. However, we did not observe significant effects of the non-Col like alleles (neither individual nor in combination with each other) on combinatorial stress response, when compared to the Col-0 like alleles. This is showing that the two alleles are not the causal alleles of HSFA1b. However, the HSFA1b gene is the most likely candidate underlying this QTL, as in our hands, the KO mutant was susceptible to drought and drought + Botrytis and the overexpression lines were tolerant to both stresses. HSFA1b belong to the HEAT SHOCK TRANSCRIPTION FACTOR (HSF) family genes and is regulated by NAC019 and flowers of nac019 plants are hypersensitive to drought stress (Duc et al., 2016; Sakuraba et al., 2015). Bechtold et al (2013) showed that overexpression HSFA1b gene led to upregulation of more than 500 genes, some of them were drought and Botrytis responsive genes. Ethylene- and JA-related genes ETHYLENE RESPONSIVE ELEMENT BINDING FACTOR 6/9 (ERF6/9), JSMONATE-ZIM-DOMAIN PROTEIN 1/5/7 (JAZ1/5/7); Botrytis defense responsive genes BCL-2-ASSOCIATED ATHANOGENE 6 (BAG6), OCTADECANOID-RESPONSIVE ARABIDOPSIS AP2/ERF 59 (ORA59); and drought responsive genes such as DRE/CRT-BINDING PROTEIN $2 B$ (DREB2b), EARLY RESPONSIVE TO DEHYDRATION 5 (ERD5), and RESPONSIVE TO DESSICATION 29B (RD29B) were up-regulated. This is showing that HSFA1b is play a role in response to Botrytis and drought stress. 


\section{Conclusion}

In this study, the univariate GWA-mapping identified a number of SNPs for combinatorial stress responses but the each SNP only explained small proportion of phenotypic variance. Follow-up analysis such as QTL mapping with biparental population would be helpful to reduce false positives and to identify QTLs with large effect. A set of 221 candidate genes associated with the significant SNPs were identified and some exhibited allelic effects on stress responses. Follow-up experiment such as complementation tests to validate the biological role of the natural alleles will be needed. Antagonistic interaction between drought and Botrytis was observed and we found the negative effect of drought pre-treatment on mutants performance and gene expression during subsequent Botrytis infection. We did not observe any mutants were tolerant to the combinatorial stress, as well as to the two single stresses. The present study provides an insights into plant response mechanisms triggered by sequential drought and Botrytis infection. We highlighted some candidate genes as targets for breeding by allele mining aimed at ultimate improvement of crop tolerance to relevant stress factor combinations. 


\section{Acknowledgements}

We are grateful to Professor Philip M Mullineaux (University of Essex, UK) for providing the over-expression lines of HSFA1b. ); We thank Prof. Maarten Koornneef and Dr. Robert Akkers for critically reading the manuscript and providing useful suggestions. This work was financially supported by The Netherlands Organization for Scientific Research (NWO) through the Technology Foundation, Perspective Programme 'Learning from Nature' [STW10988]. 


\section{Reference}

Anderson, J. P., Badruzsaufari, E., Schenk, P. M., Manners, J. M., Desmond, O. J., Ehlert, C., Maclean, D. J., Ebert, P. R. and Kazan, K. (2004). Antagonistic interaction between abscisic acid and jasmonate-ethylene signaling pathways modulates defense gene expression and disease resistance in Arabidopsis. Plant Cell 16, 3460-79.

Atkinson, N. J. and Urwin, P. E. (2012). The interaction of plant biotic and abiotic stresses: from genes to the field. J Exp Bot 63, 3523-43.

Baxter, I., Brazelton, J. N., Yu, D., Huang, Y. S., Lahner, B., Yakubova, E., Li, Y., Bergelson, J., Borevitz, J. O., Nordborg, M. et al. (2010). A coastal cline in sodium accumulation in Arabidopsis thaliana is driven by natural variation of the sodium transporter AtHKT1;1. PLoS Genet 6, e1001193.

Bechtold, U., Albihlal, W. S., Lawson, T., Fryer, M. J., Sparrow, P. A. C., Richard, F., Persad, R., Bowden, L., Hickman, R., Martin, C. et al. (2013). Arabidopsis HEAT SHOCK TRANSCRIPTION FACTORA1b overexpression enhances water productivity, resistance to drought, and infection. J Exp Bot 64, 3467-3481.

Brachi, B., Faure, N., Horton, M., Flahauw, E., Vazquez, A., Nordborg, M., Bergelson, J., Cuguen, J. and Roux, F. (2010). Linkage and association mapping of Arabidopsis thaliana flowering time in nature. PLoS Genet 6, e1000940.

Chen, Z., Hong, X., Zhang, H., Wang, Y., Li, X., Zhu, J.-K. and Gong, Z. (2005). Disruption of the cellulose synthase gene, AtCesA8/IRX1, enhances drought and osmotic stress tolerance in Arabidopsis. The Plant Journal 43, 273-283.

Dempsey, D. A., Vlot, A. C., Wildermuth, M. C. and Klessig, D. F. (2011). Salicylic Acid biosynthesis and metabolism. Arabidopsis Book 9, e0156.

Dodds, P. N. and Rathjen, J. P. (2010). Plant immunity: towards an integrated view of plant-pathogen interactions. Nat Rev Genet 11, 539-48.

Duc, H. N., Bang, H. Q. and Quang, N. X. (2016). Modelling and prediction of air pollutant transport during the 2014 biomass burning and forest fires in peninsular Southeast Asia. Environ Monit Assess 188, 106.

Franks, S. J. (2011). Plasticity and evolution in drought avoidance and escape in the annual plant Brassica rapa. New Phytol 190, 249-57.

Golisz, A., Sikorski, P. J., Kruszka, K. and Kufel, J. (2013). Arabidopsis thaliana LSM proteins function in mRNA splicing and degradation. Nucleic Acids Res $\mathbf{4 1}$, 6232-49.

Hirt, H. (2009). Plant Stress Biology: From Genomics to Systems Biology: Wiley.

Hirt, H. and Shinozaki, K. (2004). Plant Responses to Abiotic Stress: Springer.

Huang, J. L., Gu, M., Lai, Z. B., Fan, B. F., Shi, K., Zhou, Y. H., Yu, J. Q. and Chen, Z. X. (2010). Functional Analysis of the Arabidopsis PAL Gene Family in Plant Growth, Development, and Response to Environmental Stress. Plant Physiol 153, 15261538.

Kang, H. M., Sul, J. H., Service, S. K., Zaitlen, N. A., Kong, S. Y., Freimer, N. B., Sabatti, C. and Eskin, E. (2010). Variance component model to account for sample structure in genome-wide association studies. Nat Genet 42, 348-54.

Kim, W. C., Kim, J. Y., Ko, J. H., Kang, H. and Han, K. H. (2014).

Identification of direct targets of transcription factor MYB46 provides insights into the transcriptional regulation of secondary wall biosynthesis. Plant Mol Biol 85, 589-99.

Ko, J. H., Jeon, H. W., Kim, W. C., Kim, J. Y. and Han, K. H. (2014). The MYB46/MYB83-mediated transcriptional regulatory programme is a gatekeeper of secondary wall biosynthesis. Ann Bot 114, 1099-107.

Ko, J. H., Kim, W. C. and Han, K. H. (2009). Ectopic expression of MYB46 identifies transcriptional regulatory genes involved in secondary wall biosynthesis in Arabidopsis. Plant J 60, 649-65.

Kruijer, W., Boer, M. P., Malosetti, M., Flood, P. J., Engel, B., Kooke, R., Keurentjes, J. J. B. and van Eeuwijk, F. A. (2015). Marker-Based Estimation of Heritability in Immortal Populations. Genetics 199, 379-398. 
Li, Y., Huang, Y., Bergelson, J., Nordborg, M. and Borevitz, J. O. (2010). Association mapping of local climate-sensitive quantitative trait loci in Arabidopsis thaliana. Proc Natl Acad Sci U S A 107, 21199-204.

Louthan, A. M. and Kay, K. M. (2011). Comparing the adaptive landscape across trait types: larger QTL effect size in traits under biotic selection. BMC Evolutionary Biology 11, 1-12.

Mladek, C., Guger, K. and Hauser, M. T. (2003). Identification and characterization of the ARIADNE gene family in Arabidopsis. A group of putative E3 ligases. Plant Physiol 131, 27-40.

Motte, H., Vercauteren, A., Depuydt, S., Landschoot, S., Geelen, D., Werbrouck, S., Goormachtig, S., Vuylsteke, M. and Vereecke, D. (2014). Combining linkage and association mapping identifies RECEPTOR-LIKE PROTEIN KINASE1 as an essential Arabidopsis shoot regeneration gene. Proc Natl Acad Sci U S A 111, 8305-10.

Narsai, R., Wang, C., Chen, J., Wu, J., Shou, H. and Whelan, J. (2013). Antagonistic, overlapping and distinct responses to biotic stress in rice (Oryza sativa) and interactions with abiotic stress. BMC Genomics 14, 93.

Nemri, A., Atwell, S., Tarone, A. M., Huang, Y. S., Zhao, K., Studholme, D. J., Nordborg, M. and Jones, J. D. (2010). Genome-wide survey of Arabidopsis natural variation in downy mildew resistance using combined association and linkage mapping. Proc Natl Acad Sci U S A 107, 10302-7.

Onate-Sanchez, L. and Vicente-Carbajosa, J. (2008). DNA-free RNA isolation protocols for Arabidopsis thaliana, including seeds and siliques. BMC Res Notes 1, 93.

Pareek, A., Sopory, S. K. and Bohnert, H. (2010). Abiotic Stress Adaptation in Plants: Physiological, Molecular and Genomic Foundation: Springer Netherlands.

Perez-Santangelo, S., Mancini, E., Francey, L. J., Schlaen, R. G., Chernomoretz, A., Hogenesch, J. B. and Yanovsky, M. J. (2014). Role for LSM genes in the regulation of circadian rhythms. Proc Natl Acad Sci U S A 111, 15166-71.

Pieterse, C. M., Leon-Reyes, A., Van der Ent, S. and Van Wees, S. C. (2009). Networking by small-molecule hormones in plant immunity. Nat Chem Biol 5, 308-16.

Pieterse, C. M., Van der Does, D., Zamioudis, C., Leon-Reyes, A. and Van Wees, S. C. (2012). Hormonal modulation of plant immunity. Annu Rev Cell Dev Biol 28, 489-521.

Pré, M., Atallah, M., Champion, A., De Vos, M., Pieterse, C. M. J. and Memelink, J. (2008). The AP2/ERF Domain Transcription Factor ORA59 Integrates Jasmonic Acid and Ethylene Signals in Plant Defense. Plant Physiol 147, 1347-1357.

Qin, F., Sakuma, Y., Tran, L. S., Maruyama, K., Kidokoro, S., Fujita, Y., Fujita, M., Umezawa, T., Sawano, Y., Miyazono, K. et al. (2008). Arabidopsis DREB2A-interacting proteins function as RING E3 ligases and negatively regulate plant drought stress-responsive gene expression. Plant Cell 20, 1693-707.

Ramegowda, V. and Senthil-Kumar, M. (2015). The interactive effects of simultaneous biotic and abiotic stresses on plants: mechanistic understanding from drought and pathogen combination. Journal of Plant Physiology 176, 47-54.

Ramirez, V., Agorio, A., Coego, A., Garcia-Andrade, J., Hernandez, M. J., Balaguer, B., Ouwerkerk, P. B. F., Zarra, I. and Vera, P. (2011). MYB46 Modulates Disease Susceptibility to Botrytis cinerea in Arabidopsis. Plant Physiol 155, 1920-1935.

Sadhukhan, A., Panda, S. K. and Sahoo, L. (2014). The cowpea RING ubiquitin ligase VuDRIP interacts with transcription factor VuDREB2A for regulating abiotic stress responses. Plant Physiol Biochem 83, 51-6.

Sakuraba, Y., Kim, Y.-S., Han, S.-H., Lee, B.-D. and Paek, N.-C. (2015). The Arabidopsis Transcription Factor NAC016 Promotes Drought Stress Responses by Repressing AREB1 Transcription through a Trifurcate Feed-Forward Regulatory Loop Involving NAP. Plant Cell 27, 1771-1787.

Sewelam, N., Oshima, Y., Mitsuda, N. and Ohme-Takagi, M. (2014). A step towards understanding plant responses to multiple environmental stresses: a genomewide study. Plant Cell Environ 37, 2024-35. 
Shaik, R. and Ramakrishna, W. (2013). Genes and co-expression modules common to drought and bacterial stress responses in Arabidopsis and rice. PLOS ONE 8 , e77261.

Sherrard, M. E. and Maherali, H. (2006). The adaptive significance of drought escape in Avena barbata, an annual grass. Evolution 60, 2478-89.

Shinozaki, K. and Yamaguchi-Shinozaki, K. (2007). Gene networks involved in drought stress response and tolerance. J Exp Bot 58, 221-7.

Sokal RR, R. F. (1981). Biometry the Principles and Practice of Statistics in Biological Research (2nd ed.). . WH Freeman and Company, San Francisco, USA.

Staats, M. and Van Kan, J. A. L. (2012). Genome update of Botrytis cinerea strains B05.10 and T4. Eukaryotic Cell 11, 1413-1414.

Sterken, R., Kiekens, R., Boruc, J., Zhang, F., Vercauteren, A., Vercauteren, I., De Smet, L., Dhondt, S., Inze, D., De Veylder, L. et al. (2012). Combined linkage and association mapping reveals CYCD5;1 as a quantitative trait gene for endoreduplication in Arabidopsis. Proc Natl Acad Sci U S A 109, 4678-83.

Sun, G., Zhu, C., Kramer, M. H., Yang, S. S., Song, W., Piepho, H. P. and Yu, J. (2010). Variation explained in mixed-model association mapping. 105, 333-340.

Van Wees, S. C. M., Van Pelt, J. A., Bakker, P. A. H. M. and Pieterse, C. M. J. (2013). Bioassays for assessing jasmonate-dependent defenses triggered by pathogens, herbivorous Insects, or beneficial rhizobacteria. Methods in Molecular Biology 1011, 35-49.

Vos, I. A., Verhage, A., Schuurink, R. C., Watt, L. G., Pieterse, C. M. J. and Van Wees, S. C. M. (2013). Onset of herbivore-induced resistance in systemic tissue primed for jasmonate-dependent defenses is activated by abscisic acid. Frontiers in Plant Science 4, 539.

Xiong, L., Gong, Z., Rock, C. D., Subramanian, S., Guo, Y., Xu, W., Galbraith, D. and Zhu, J. K. (2001). Modulation of abscisic acid signal transduction and biosynthesis by an Sm-like protein in Arabidopsis. Dev Cell 1, 771-81.

Yoshioka, K. and Shinozaki, K. (2009). Signal Crosstalk in Plant Stress Responses: Wiley.

Zehrmann, A., Hartel, B., Glass, F., Bayer-Csaszar, E., Obata, T., Meyer, E., Brennicke, A. and Takenaka, M. (2015). Selective homo- and heteromer interactions between the multiple organellar RNA editing factor (MORF) proteins in Arabidopsis thaliana. J Biol Chem 290, 6445-56.

Zhou, J., Lee, C., Zhong, R. and Ye, Z. H. (2009). MYB58 and MYB63 are transcriptional activators of the lignin biosynthetic pathway during secondary cell wall formation in Arabidopsis. Plant Cell 21, 248-66. 


\section{Supporting Information}

Additional supporting information may be download via link

https://www.dropbox.com/sh/hb95t5dwvxbsc1u/AAB2Z7Q1DPSwCCUoaTBBi2Pa?dl=0 



\section{$\mathbb{C} \| \Delta \mathbb{P} \mathbb{I} \mathbb{E} \mathbb{R} 5$}

\section{Transcriptome dynamics of Arabidopsis during sequential biotic and abiotic stresses}

Silvia Coolen ${ }^{1 *}$, Silvia Proietti ${ }^{1 *}$, Richard Hickman ${ }^{1 *}$, Nelson H. Davila Olivas ${ }^{2 *}$, Ping-Ping Huang ${ }^{3 *}$, Marcel C. Van Verk ${ }^{1,4}$, Johan A. Van Pelt ${ }^{1}$, Alexander H.J. Wittenberg ${ }^{5}$, Martin De Vos $^{5}$, Marcel Prins ${ }^{5}$, Joop J.A. Van Loon ${ }^{2}$, Mark G.M. Aarts $^{3}$, Marcel Dicke ${ }^{2}$, Corné M.J. Pieterse ${ }^{1}$ and Saskia C.M. Van Wees ${ }^{1}$

Plant J, (2016), 86: 249-267. doi:10.1111/tpj.13167JJ,

* These authors contributed equally to this work.

${ }^{1}$ Plant-Microbe Interactions, Department of Biology, Utrecht University, P.O. Box 800.56, 3508 TB, Utrecht, the Netherlands

${ }^{2}$ Laboratory of Entomology, Wageningen University, P.O. Box 16, 6700 AA Wageningen, the Netherlands

${ }^{3}$ Laboratory of Genetics, Wageningen University, P.O. Box 16, 6700 AA Wageningen, the Netherlands

${ }^{4}$ Bioinformatics, Department of Biology, Utrecht University, P.O. Box 800.56, 3508 TB, Utrecht, the Netherlands

${ }^{5}$ Keygene N.V., P.O. Box 216, 6700 AE Wageningen, the Netherlands. 


\section{Abstract}

In nature, plants have to cope with a wide range of stress conditions that often occur simultaneously or in sequence. To investigate how plants cope with multi-stress conditions, we analyzed the dynamics of whole-transcriptome profiles of Arabidopsis thaliana exposed to six sequential double stresses inflicted by combinations of (1) infection by the fungus Botrytis cinerea, (2) herbivory by Pieris rapae, and (3) drought stress. Each of these stresses induced specific expression profiles over time, in which one third of all differentially expressed genes was shared by at least two single stresses. Of these, 394 genes were differentially expressed during all three stress conditions, albeit often in opposite directions. When two stresses were applied in sequence, plants displayed transcriptome profiles that were very similar to the second stress, irrespective of the nature of the first stress. Nevertheless, significant first-stresssignatures could be identified in the sequential stress profiles. Bioinformatic analysis of the dynamics of co-expressed gene clusters highlighted specific clusters and biological processes of which the timing of activation or repression was altered by a prior stress. The first-stress-signatures in second stress transcriptional profiles were remarkably often related to responses to phytohormones, strengthening the notion that hormones are global modulators of interactions between different types of stress. Because prior stresses can affect the level of tolerance against a subsequent stress (e.g. prior herbivory strongly affected $B$. cinerea resistance), the first-stress-signatures can provide important leads for the identification of molecular players that are decisive in the interactions between stress response pathways.

\section{Keywords:}

Combinatorial plant stress, transcript profiling, Botrytis cinerea, Pieris rapae, drought stress, gene regulatory network, plant hormones, RNA-Seq, Arabidopsis thaliana 


\section{Introduction}

Plants are continuously threatened by a wide range of harmful microbial pathogens and insect herbivores. Besides these biotic stresses, plants are also exposed to extreme abiotic environmental conditions such as drought, heat, cold, water logging, high salinity or toxicity. Adaptive plant responses to single biotic and abiotic stresses have been extensively studied. Both biotic and abiotic stress responses are associated with the action of the phytohormones jasmonic acid $(J A)$, ethylene (ET), abscisic acid (ABA), and salicylic acid (SA), and to a lesser extent with cytokinin, brassinosteroids and auxin (Broekgaarden et al. 2015, Giron et al. 2013, Kazan and Lyons 2014, O'Brien and Benková 2013, Pieterse et al. 2012, Robert-Seilaniantz et al. 2011). JA and ET are generally involved in defense against pathogens with a necrotrophic lifestyle, whereas defenses against biotrophs are commonly controlled by SA (Glazebrook 2005). ABA is associated with plant development and abiotic stresses (Yamaguchi-Shinozaki and Shinozaki 2006), such as drought, but its role in modulating JA-dependent defenses against insect herbivores and SA-dependent defenses against pathogens is becoming increasingly evident (Verhage et al. 2011, Vos et al. 2013b, Yasuda et al. 2008). Antagonistic and synergistic interactions between hormonal signal-transduction pathways are thought to provide the plant with a regulatory potential to adapt to its complex biotic and abiotic environment while utilizing its resources in a cost-efficient manner (Pieterse et al. 2012, Reymond and Farmer 1998, Robert-Seilaniantz et al. 2011, Vos et al. 2015, Vos et al. 2013a).

In natural and agricultural settings, plants often have to cope with multiple stress conditions simultaneously. In the context of climate change, it is highly likely that the frequency and complexity of these multi-stress conditions will increase and further threaten crop yield. Abiotic stresses can significantly affect plant responses to biotic stresses and vice versa, depending on the timing, nature, and severity of the stresses (Appel et al. 2014, Atkinson and Urwin 2012, Rejeb et al. 2014). How plants regulate and prioritize their adaptive response when exposed to multiple stresses is largely unknown. Several studies have investigated plant responses to different stress factors occurring simultaneously or sequentially (Atkinson et al. 2013, De Vos et al. 2006, Kissoudis et al. 2014, 
Mohr and Cahill 2003, Prasch and Sonnewald 2013, Ramegowda and SenthilKumar 2015, Rasmussen et al. 2013, Rivero et al. 2014, Santino et al. 2013, Sewelam et al. 2014, Sham et al. 2015, Stam et al. 2014, Suzuki et al. 2014, Van Oosten et al. 2008). From these studies, the picture emerged that different stress signaling pathways are interconnected in a network that is under control of key regulators such as MAP kinases, transcription factors and the abovementioned stress-related hormones (Caarls et al. 2015, Fujita et al. 2006, Pieterse et al. 2009, Rejeb et al. 2014, Robert-Seilaniantz et al. 2011). In order to gain insight in the complexity of the plant response to combinatorial stresses, several recent studies investigated changes in the transcriptome of Arabidopsis thaliana (hereafter called Arabidopsis) in response to simultaneous exposure to abiotic and biotic stresses (Atkinson et al. 2013, Prasch and Sonnewald 2013, Ramegowda and Senthil-Kumar 2015, Rasmussen et al. 2013, Sham et al. 2015, Suzuki et al. 2014). Generally, the responses to the single stresses were different from those to the double stresses. However, these studies often focused on a single time point, representing only a snapshot of the transcriptional changes that are induced by a single or combinatorial stress. The influence of one stress on the other may primarily have an effect on the timing of the response to the second stress, causing the detection of large transcriptional differences in combinatorial stresses at one time point, while over time these differences may be much smaller or are the result of a shift in the phasing of the expression profiles.

In order to gain detailed insight into how plants cope with multiple stresses, we here investigated how a first stress influences the nature and dynamics of the transcriptional response that is induced by a second stress. We chose to study the response of the model plant species Arabidopsis to two biotic stresses (infection by the necrotrophic fungus Botrytis cinerea and herbivory by larvae of Pieris rapae) and to one abiotic stress (drought stress by water withhold). These stresses were chosen because in previous studies it was demonstrated that the plant hormones JA, ABA, and/or ET are involved in adaptive plant responses to these respective stresses. We hypothesized that combining these stresses may lead to hormonal signal interactions that potentially affect the outcome of the response to the second stress. Several 
previous studies have identified thousands of Arabidopsis genes that change in expression in response to the selected single stresses (Birkenbihl et al. 2012, Clauw et al. 2015, De Vos et al. 2005, Ferrari et al. 2007, Huang et al. 2008, Rehrig et al. 2014, Reymond et al. 2004, Reymond et al. 2000, Rowe et al. 2010, Windram et al. 2012), but their dynamic behavior during multi-stress conditions is largely unknown.

B. cinerea is considered the second most important plant pathogen (Dean et al. 2012), infecting over 200 cultivated plant species and causing significant economic damage to crops worldwide. Moreover, $B$. cinerea has become an important model for studying interactions between plants and necrotrophic pathogens (Laluk and Mengiste 2010, Van Kan 2006). As a necrotroph, $B$. cinerea kills plant tissue prior to feeding by using different mechanisms that cause plant decay, e.g. enzymatic degradation of the cell walls, generation of toxic reactive oxygen compounds, or secretion of host nonselective toxins. JA and ET participate in the defense response of Arabidopsis against B. cinerea (Diaz et al. 2002, El Oirdi et al. 2011, Geraats et al. 2002, Rowe et al. 2010, Thomma et al. 1998, Thomma et al. 1999), while ABA and SA can have a negative effect on $B$. cinerea resistance (El Oirdi et al. 2011, Liu et al. 2015, Vos et al. 2015).

Insect herbivores consume over $15 \%$ of the plant biomass produced annually in temperate and tropical ecosystems making insect herbivory a major conduit by which energy flows through food webs (Agrawal 2011, Cyr and Pace 1993, Johnson 2011). The Small Cabbage White butterfly $P$. rapae is one of the most destructive pests of cruciferous plants because it has adapted to the glycoside toxins known as glucosinolates that are produced by crucifers as chemical defenses (Hopkins et al. 2009). Arabidopsis and other plants activate additional defense responses that reduce the performance of leaf-chewing $P$. rapae caterpillars on pre-infested plants (De Vos et al. 2006). It has been shown that this herbivore- or wound-induced resistance also extends systemically to undamaged plant parts (Howe and Jander 2008, Vos et al. 2013b). JA is an important primary signal in herbivore-induced local and systemic defenses in various plant-herbivore interactions, while ABA has a modulating role in the JA-responsiveness (Bodenhausen and Reymond 2007, 
Howe and Jander 2008, Soler et al. 2013, Vos et al. 2013b). SA is reported to inhibit the JA-dependent defense pathway that is induced by $P$. rapae feeding (Koornneef et al. 2008).

Drought is one of the most frequently experienced abiotic environmental stresses in plants. Low water availability in the rhizosphere leads to a reduction in leaf stomatal conductance and growth (Schachtman and Goodger 2008). Adaptive responses to drought also involve metabolic, osmotic, and structural adjustment, as well as the production of proteins with DNA damage control and repair functions (Ingram and Bartels 1996). ABA accumulation is essential for the adaptation to drought, but also $A B A$-independent regulatory systems are involved in drought stress-responsive gene expression. In the latter, JA and ET have been implicated as important regulators (Bray 1997, Huang et al. 2008, Riera et al. 2005, Shinozaki et al. 2003).

In this study, we used RNA-Seq to analyze the dynamics of the transcriptome changes that occurred in Arabidopsis over four time points in response to $B$. cinerea infection, $P$. rapae feeding, drought stress, and all six combinations of sequential double stresses. Our results show that irrespective of the first stress, Arabidopsis is capable of swiftly adapting its transcriptome to respond to the second stress. Over time, this second stress-induced transcriptome is highly similar to that of plants that did not receive a first stress, but contains clear first-stress-signatures, which may play a role in the phenotypic interaction between consecutive stresses. 


\section{Material \&Methods}

\section{Plant cultivation}

Seeds of Arabidopsis thaliana accession Col-0 were sown in cultivation containers filled with autoclaved river sand. Sand was supplied with halfstrength Hoagland solution containing Sequestreen as described (Van Wees et al. 2013). In order to attain $100 \%$ relative humidity $(\mathrm{RH})$ for germination, cultivation containers were enclosed in a tray with water covered with a transparent lid. Seeds were stratified for two days at $4^{\circ} \mathrm{C}$ in the dark to ensure a homogeneous germination after which the tray was moved to a growth chamber $(\mathrm{t}=0)$ with an 8-h day/16-h night rhythm, a temperature of $21^{\circ} \mathrm{C}$, and a light intensity of $100 \mu \mathrm{mol} / \mathrm{m}^{2} / \mathrm{s}$. After $10 \mathrm{~d}$, the lids of the trays were slightly opened and gradually removed over a 4 -d period to adjust to the $70 \% \mathrm{RH}$ present in the growth chamber. Fourteen-day-old seedlings were transplanted to individual pots containing a mixture of river sand and potting soil (1:1 (v:v)). Pots were supplied with water from the bottom up three times per week. At an age of 3 weeks the plants were supplied once with half-strength Hoagland solution.

\section{Rearing of $P$. rapae and treatment with $P$. rapae caterpillars}

Pieris rapae caterpillars were reared on cabbage plants (Brassica oleracea convar. capitata var. alba) under greenhouse conditions $\left(24^{\circ} \mathrm{C}\right.$, with natural daylight). Butterflies were supplied with flowering plants such as Lantana camara for their (nectar) food. When flowers were scarce, additional food (solution of $20 \%$ honey and $10 \%$ sucrose) was offered to the butterflies. Inbreeding of the population was minimalized by adding wild butterflies and caterpillars from the Dutch Flevopolder to the existing population. After starving for $1 \mathrm{~h}$, first-instar (L1) larvae were placed on Arabidopsis leaves using a fine paint brush as described (Van Wees et al. 2013).

\section{Cultivation of B. cinerea and treatment with B. cinerea spores}

Botrytis cinerea strain B05.10 (Staats and Van Kan 2012) was grown on half-strength Potato Dextrose Agar (PDA; Difco Laboratories) plates containing penicillin $(100 \mu \mathrm{g} / \mathrm{ml})$ and streptomycin $(200 \mu \mathrm{g} / \mathrm{ml})$ for 2 weeks at room 
temperature. Spores were collected, filtered through glass wool, and resuspended in half-strength Potato Dextrose Broth (PDB; Difco Laboratories) to a final density of $1 \times 10^{5}$ spores/ml. After a 3-h incubation period, the spores were used for inoculation by applying 5- $\mu$ l droplets on Arabidopsis leaves as described (Van Wees et al. 2013). For the RNA-Seq analysis, 4 droplets were applied on a single leaf, while for disease resistance assays, a single droplet was administered to the leaf.

\section{Single and sequential double stress treatments}

Single and sequential double stress treatments were applied according to the schedule shown in Fig. 1. Developmental leaf number 8 was treated with the second stress and harvested for RNA-Seq analysis. Individual leaves were numbered from oldest to youngest. For single and sequential double stress treatments in which $B$. cinerea was the second stress, developmental leaf number 8 of 5 -week-old plants was inoculated with $B$. cinerea by pipetting four $5-\mu l$ droplets of spore suspension $\left(1 \times 10^{5}\right.$ spores $\left./ \mathrm{ml}\right)$ onto the leaf. Plants were kept at $100 \% \mathrm{RH}$ for the remaining time period. Mock-treated plants received droplets of PDB and were kept at $100 \% \mathrm{RH}$. Pre-treatment with drought was started when plants were 4 weeks old by withholding water for $7 \mathrm{~d}$, after which plants were re-watered and allowed to recover for $1 \mathrm{~d}$ before plants were inoculated with $B$. cinerea. $P$. rapae pre-treatment was started $1 \mathrm{~d}$ prior to $B$. cinerea inoculation by placing a single $P$. rapae $\mathrm{L} 1$ caterpillar on leaf 7 and allowing it to feed for $1 \mathrm{~d}$. Only plants of which leaf number 8 was undamaged were used for inoculation with $B$. cinerea as second stress. Leaf number 8 was harvested at $6,12,18$, and $24 \mathrm{~h}$ after inoculation with $B$. cinerea.

For single and sequential double stress treatments in which $P$. rapae herbivory was the second stress, two $P$. rapae $L 1$ larvae were transferred to developmental leaf number 8 of 5 -week-old plants. Pre-treatment with drought was achieved as described above for the $B$. cinerea experimental set-up. $B$. cinerea pre-treatment was performed $1 \mathrm{~d}$ prior to introduction of $P$. rapae by inoculating leaves 6 and 7 with one $5-\mu$ droplet of $B$. cinerea spore suspension $\left(1 \times 10^{5}\right.$ spores $\left./ \mathrm{ml}\right)$ per leaf and placing the plants at $100 \% \mathrm{RH}$ for $1 \mathrm{~d}$. A mock treatment for the $B$. cinerea pre-treatment was included by placing droplets with 
PDB on the leaves and keeping the plants at $100 \% \mathrm{RH}$ for $1 \mathrm{~d}$. Leaf number 8 was harvested at 3, 6, 12 and $24 \mathrm{~h}$ after the start of $P$. rapae feeding. When leaf number 8 was not damaged by $P$. rapae (because it had moved to another leaf), the next-closest $P$. rapae-damaged leaf was harvested.

For single and sequential double stress treatments in which drought was the second stress, 4-week-old plants were refrained from watering for $7 \mathrm{~d}$. After $7 \mathrm{~d}$ of water withhold, plants were re-watered and allowed to recover for $1 \mathrm{~d}$. $B$. cinerea pre-treatment was performed at the beginning of day 1 of the drought period by inoculating leaves 6 and 7 with one $5-\mu$ droplet of $B$. cinerea spore suspension $\left(1 \times 10^{5}\right.$ spores $\left./ \mathrm{ml}\right)$ per leaf and placing the plants at $100 \% \mathrm{RH}$ for 1 d. A mock treatment for the $B$. cinerea pre-treatment was included by placing droplets with PDB on the leaves and keeping the plants at $100 \% \mathrm{RH}$ for $1 \mathrm{~d}$. $P$. rapae pre-treatment was performed at the same time as the $B$. cinerea pretreatment by placing one $P$. rapae caterpillar on leaf 7 and allowing it to feed on the plant for $1 \mathrm{~d}$. Only plants of which leaf number 8 was undamaged were used to harvest leaf 8 . Leaf number 8 was harvested at $5,6,7$, and $7+1 \mathrm{~d}$ after the onset of water withhold (with $7+1$ representing the time point of $1 \mathrm{~d}$ after rewatering).

For each treatment and time point, 3 biological replicates were used for RNA-Seq analysis. Each of the three biological replicates consisted of four pooled "number 8" leaves harvested from four similarly-treated plants. For all treatments in which $B$. cinerea inoculation was used as first or second stress, a mock treatment was performed in which plants were inoculated with droplets of half-strength PDB and placed at $100 \%$ RH for 1 d. For all treatments without $B$. cinerea, controls consisted of untreated plants. After harvest, leaf samples were immediately frozen in liquid nitrogen and stored at $-80^{\circ} \mathrm{C}$. 


\section{Experimental design}

The experiment was carried out in a fully randomized factorial design with two factors; time and treatment, with time having four levels (four time points analyzed per stress combination) and treatment having five levels (control, mock, two different first stresses per sequential stress, and one single stress). The climate chamber space was divided in three blocks, in which time was randomized. Within every time point, treatments were assigned randomly to the plants. RNA extraction was carried out in batches of approximately 20 randomly chosen samples.

\section{RNA extraction, library preparation, and RNA-Seq alignment}

RNA was extracted using the Plant RNeasy Plant Mini Kit (Qiagen), according to the manufacturer's instructions. All samples were treated with DNase I on column using the Qiagen RNase-Free DNase Set. Quality of RNA was checked by determining the RNA Integrity Number (RIN) with an Agilent 2100 bioanalyzer and RNA LabChip. For the library preparation samples with a RIN value $\geq 6$ were used. The samples were processed according to the TruSeq Stranded mRNA HT Sample Prep Kit from Illumina. This protocol allows the identification of strandspecific transcripts. First, poly-A RNA was isolated from the total RNA using Poly$\mathrm{T}$ oligo-attached magnetic beads. Subsequently, Poly-A RNA was fragmented using divalent cations under elevated temperature. First-strand cDNA was synthesized using random primers. Strand specificity was achieved by replacing dTTP with dUTP in the second Strand Marking Mix (SMM), followed by second strand CDNA synthesis using DNA polymerase I and RNase H. Samples were sequenced with an Illumina Hi-seq 2000 sequencer using three sequencing runs. Samples were randomly assigned to 7 lanes of the Illumina flow cells within each run.

Processing of raw sequencing data, alignment of the RNA-Seq data to the Arabidopsis genome, and downstream processing was performed as described (Van Verk et al. 2013). RNA-Seq reads were aligned to the Arabidopsis genome (TAIR version 10) using TopHat v2.0.4 (Trapnell et al. 2009) with parameters: 'transcriptome-mismatches 3', 'N 3', 'bowtie1', 'no-noveljuncs', 'genome-read-mismatches 3', 'read-mismatches 3', 'G', 'min-intron- 
length 40 ', 'max-intron-length 2000'. Gene expression levels were calculated by counting the number of mapped reads per annotated gene model using HTSeqcount v0.5.3p9 (Anders et al. 2014). For downstream analyses, raw read counts were normalized for between sample differences in sequencing depth (Love et al. 2014). Differential gene expression was calculated using DESeq2 (Love et al. 2014) for all stress treatments and time points relative to the appropriate nonstress-treated control/mock treatment that was cultivated and harvested in exactly the same way as the stress-treated samples. The raw $P$. rapae RNA-Seq data have been used in a previous study (Davila Olivas et al. 2016). In the study of Davila Olivas et al., the raw $P$. rapae RNA-Seq reads were analyzed independently of this study with the specific goal to identify $P$. rapae-responsive genes that are affected by prior drought stress or $B$. cinerea infection and possibly link them to effects on changes in $P$. rapae resistance. In the present study, the raw $P$. rapae RNA-Seq reads were used in the larger framework of analyzing global dynamics of gene expression profiles during multiple combinatorial stress conditions in which also $B$. cinerea and drought stress were analyzed as second stresses. All raw RNA-Seq read data are deposited in the NCBI Short Read Archive (http:// www.ncbi.nlm.nih.gov/sra/) under the BioProject accession code PRJNA315516.

\section{Gene ontology analysis}

To identify enrichment of gene ontology (GO)-terms in the different sets of DEGs, 'Go term finder' (Boyle et al. 2004) analysis was performed using an $A$. thaliana gene association file downloaded from ftp.geneontology.org on May $2^{\text {nd }}$ 2013. The default background set was used (all 30504 transcripts in the database that have GO annotations). GO term finder tests for overrepresentation of $\mathrm{GO}$ categories using the hypergeometric distribution and false discovery rate for multiple testing ( $P$-value $\leq 0.05)$. Figures showing heatmaps of $P$-values were generated using the R package (version 3.2.1). 


\section{Clustering}

Hierarchical clustering of the core set of single stress DEGs was performed on $\log _{2}$ fold-change expression values using the $\mathrm{R}$ function hclust with a cosine similarity metric and average linkage. The cutree function was used with a visually determined cut height to partition the resulting dendrogram into clusters. Clustering of the core set of single stress DEGs and shared main treatment datasets was performed using model-based clustering package mclust version 4 in R (Fraley et al. 2012) with the number of clusters optimized in the range 1 to 10 using the Bayesian information criterion.

\section{Wigwams analysis}

To identify modules of co-expressed genes across single and sequential stresses the Wigwams algorithm was applied (Polanski et al. 2014), using $\log _{2}$ transformed expression values for the DEGs of each single stress across their respective sequential double stresses. For each main treatment, Wigwams was run with default arguments to partition genes into modules that indicate coexpression in subsets of the relevant main treatments. 


\section{Results}

\section{Experimental approach for RNA-Seq analysis of single and sequential stress time series}

In order to capture a maximal dynamic range of the stress responses, the response to each of the three main stresses was monitored in a different time frame of four time points, depending on how quickly the stress response developed (Fig. 1).

The transcriptional response to each single and sequential stress was compared at each time point to a non-treated control (for treatments not involving $B$. cinerea) or a mock-treated control (same $100 \%$ relative humidity conditions as $B$. cinerea treatments) that was harvested at the same time as the stress treatment. For the study of $B$. cinerea stress, a time span between 6 and $24 \mathrm{~h}$ after inoculation with a $5-\mu \mathrm{l}$ droplet of $5 \times 10^{5}$ spores $/ \mathrm{ml}$ was chosen, because previous studies showed that the earliest transcriptional changes can be observed around $6 \mathrm{~h}$ after application of the inoculum, while at $24 \mathrm{~h}$ after inoculation massive changes in gene expression can be detected (Vos et al. 2015, Windram et al. 2012). For the study of $P$. rapae stress, we chose a time span between 3 and $24 \mathrm{~h}$ after infestation by larvae of stage L1 because previous studies demonstrated that this would yield a maximal dynamic range of transcriptional responses (De Vos et al. 2005, Reymond et al. 2004, Reymond et al. 2000, Verhage et al. 2011). For the induction of drought stress, 4-week-old Arabidopsis plants that had previously been watered with equal amounts of water were subsequently withheld from water for 7 days. At day 5 of water withhold, drought-stressed plants were clearly smaller and darker colored than the watered control plants, a phenotype that progressed further on day 6 and 7 when they were at the point of wilting. The transcriptome time series were chosen at 5, 6 and 7 days after water withhold, and at day $8(7+1 d)$, which was one day after re-watering. The recovery response at day 8 was chosen as the fourth time point of the drought time series because this recovery response after drought stress is interesting by itself, and at this time point the sequential treatment with $B$. cinerea and $P$. rapae was executed and thus could function as 
a reference treatement. Prior to applying the second stress, further development of the first stress was stopped by changing the $100 \%$ relative humidity condition to $70 \%$ (first stress $B$. cinerea), by removing the caterpillar (first stress $P$. rapae), or by re-watering the plants (first stress drought). Developmental leaf number 8 was used for applying B. cinerea or $P$. rapae as second stress. For all treatments, leaf number 8 was harvested for RNA-Seq analysis. When leaf number 8 was not damaged by $P$. rapae, the next-closest $P$. rapae-damaged leaf was harvested. Three biological replicates per treatment and time point were subjected to RNA-Seq. Each of the three biological replicates consisted of four "number 8 " leaves that were pooled to form one sample. After harvest, leaves were processed and subjected to RNA-Illumina sequencing. On average, 14.6 million reads (range $8.5-29.8$ million) were generated per sample with $>90 \%$ of sequences aligning to the Arabidopsis genome after quality filtering (Van Verk et al. 2013). 


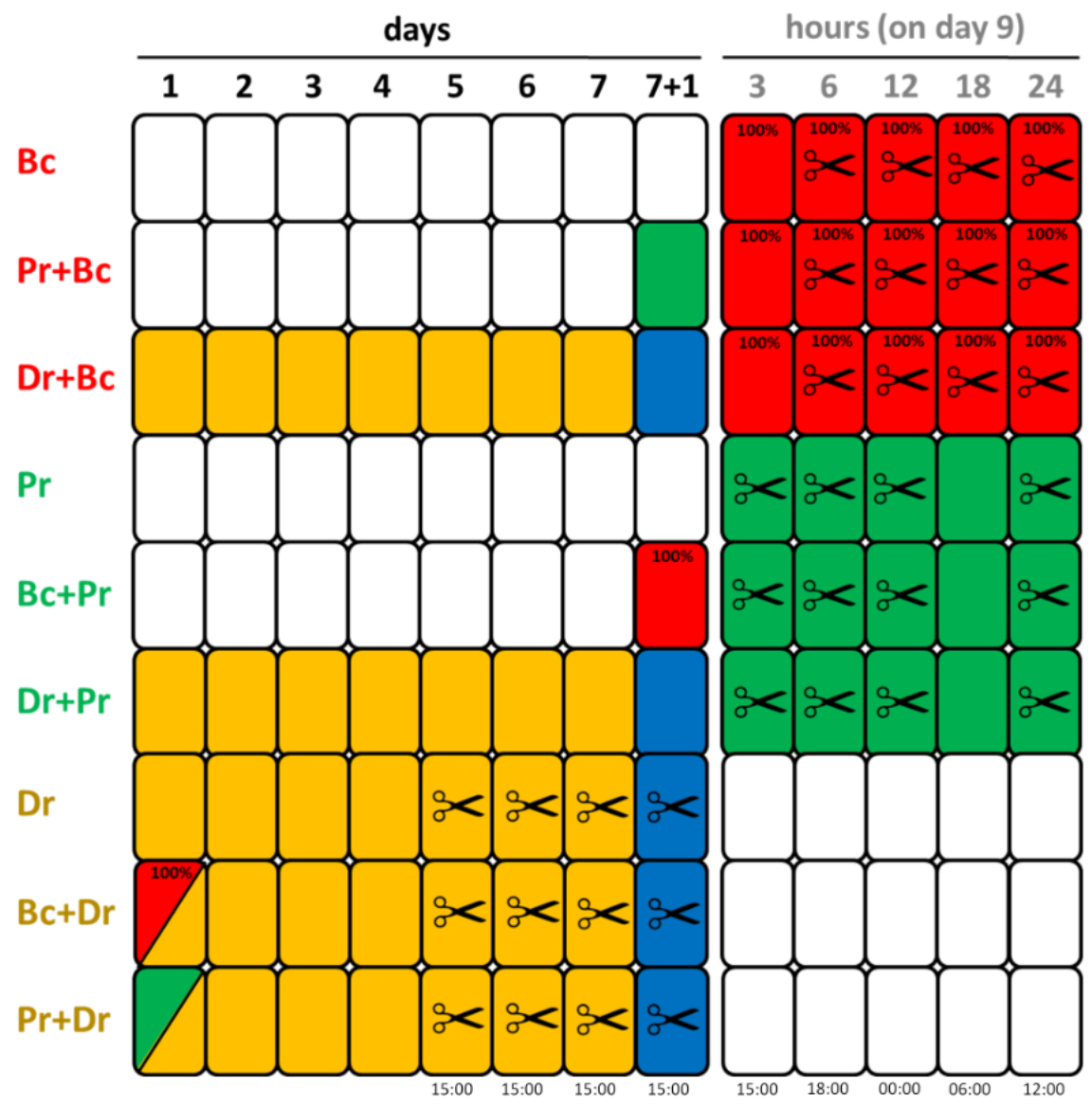

Figure 1. Experimental schedule of treatments and harvests for RNA-Seq time series of single and sequential double stresses. The schedule shows the timing of treatments and time points of harvest for the three main treatments, $B$. cinerea $(\mathrm{Bc}$, red), $P$. rapae ( $\mathrm{Pr}$, green) and Drought ( $\mathrm{Dr}$, yellow), and the respective pre-treatments. Each single and sequential double stress sample had a mock/control (not visualized) that was harvested at the same time point as the stress treatment. Mock-treated plants were cultivated under the same conditions as their respective $B$. cinerea-treated plants (same periods of $100 \% \mathrm{RH}$ ). Untreated control plants were cultivated under the same conditions as their respective $P$. rapae- and/or drought-treated plants. First stresses were stopped by either lowering relative humidity from $100 \%$ to $70 \%$ (after $1 \mathrm{~d}$ in case of Bc pretreatment), removing caterpillars from plants (after $1 \mathrm{~d}$ ), or re-watering after a 7-d period of drought $(7+1 ;$ blue). In case the second stress was drought, the pre-treatments with $B$. cinerea and $P$. rapae were performed right after the last moment of watering. $100 \%$; period of $100 \% \mathrm{RH}$ instead of standard $70 \% \mathrm{RH}$; time indications at the bottom indicate time of the day at which plants were harvested. 


\section{Time series transcriptome profiling following single and sequential stresses}

In this study, our aim was to analyze the dynamic transcriptome changes that are triggered by the single stresses and investigate how the nature and dynamics of these transcriptome profiles were affected by pre-exposure to each of the other two stresses. First, a set of differentially expressed genes (DEGs) derived from each single stress time series was selected according to their significance in fold-change expression (false discovery rate (FDR) $<0.05$ ) and an additional threshold level of at least 2 -fold change $\left(-1>\log _{2}>1\right)$ in comparison to the respective control (Supporting Table S1). The first observation that can be made from the RNA-Seq results is that over time there are clear differences in the number of genes that are significantly activated or repressed during the different single stress conditions (Fig. 2). For responses to B. cinerea (total 2076 unique DEGs) and $P$. rapae (total 3952 unique DEGs), a strong increase in the number of activated genes is observed over time, while relatively few genes are repressed. Upon exposure to drought stress (total 4032 unique DEGs for the first three time points, plus 482 additional unique DEGs for the $1 \mathrm{~d}$ after rewatering time point), relatively more genes become repressed than activated. A prior stress did not dramatically change the number of DEGs relative to the single stresses (Fig. 2). Clustering the union of DEGs of the single stress sets (total 7355 unique DEGs), and subsequent Gene Ontology (GO) analysis (Boyle et al. 2004) of overrepresented biological processes in each cluster highlights the differentially regulated biological processes during the plant response to the single stresses and uncovers similarities and contrasts between the different stress responses (Fig. 3; Supporting Table S2). 

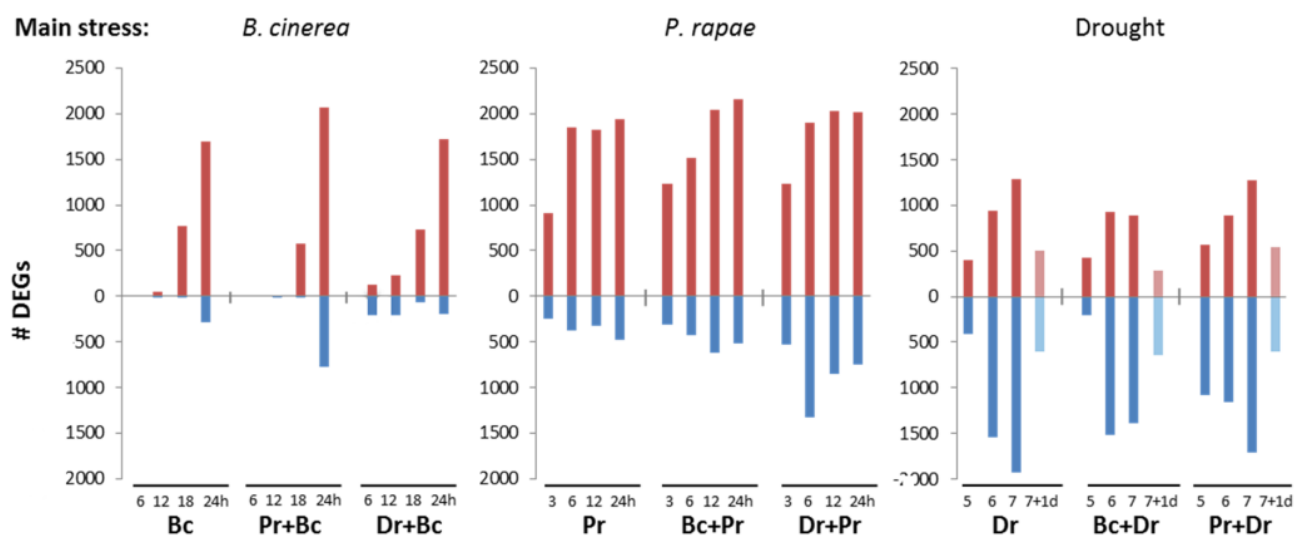

Figure 2. Numbers of DEGs at different time points in single and sequential stress responses. Graphs show the number of activated (red bars) and repressed (blue bars) differentially expressed genes (DEGs) for all single stresses and their corresponding sequential double stresses at different time points after treatment (FDR $<0.05$; $>2$-fold). The one day after rewatering time point of the drought treatments is indicated as " $7+1 \mathrm{~d}$ ". $\mathrm{Bc}$, B. cinerea; $\mathrm{Pr}, \mathrm{P}$. rapae; $\mathrm{Dr}$, drought; $\mathrm{Pr}+\mathrm{Bc}, \mathrm{Dr}+\mathrm{Bc}, \mathrm{Bc}+\mathrm{Pr}, \mathrm{Dr}+\mathrm{Pr}, \mathrm{Bc}+\mathrm{Dr}$, and $\mathrm{Pr}+\mathrm{Dr}$, respective sequential double stresses.

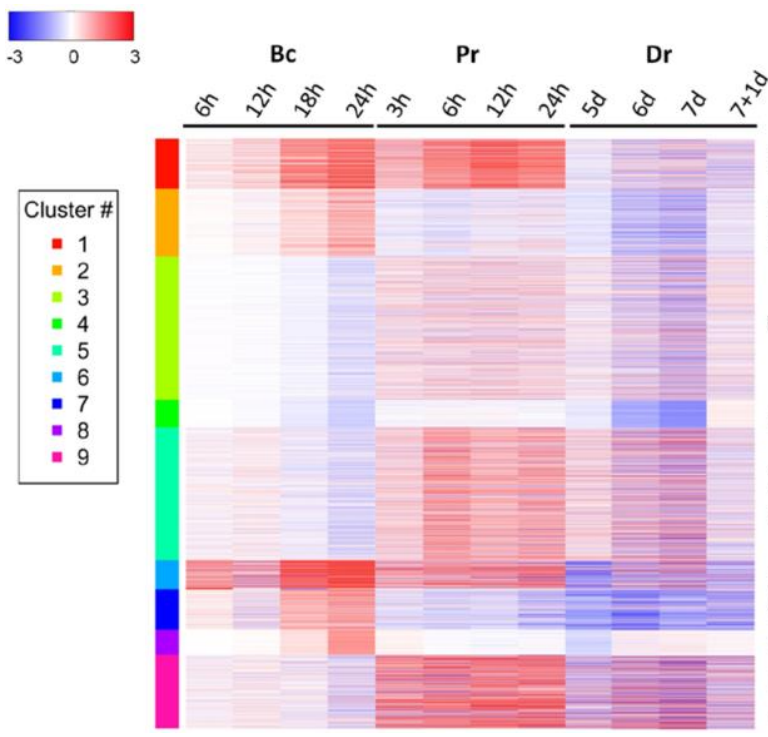

Response to chitin
Defense response,
incompatible interaction
Response to stimulus
Photosynthesis
Cell wall macromolecule
metabolic process
Response to chemical stimulus
Systemic acquired resistance
RNA methylation
Response to oxygen
containing compound

Figure 3. Clustering of the single stress DEGs. Heatmap showing the expression patterns of the union of differentially expressed genes (DEGs) in the three single stresses at different time points after induction (total 7173 unique genes). DEGs were clustered using Mclust yielding 9 gene clusters (colored bars on the left). On the right side, the most significant GO term for each cluster (full data set in Supporting Table S2). Bc, B. cinerea; $\mathrm{Pr}, P$. rapae; Dr, drought. For drought stress, the time point one day after re-watering $(7+1 d)$ was included in the cluster analysis. Blue-red color key for change in gene expression level: $-3>\log _{2}$ fold change $>3$. 


\section{Core DEGs that are shared between the single stress responses}

To investigate to what extent genes and biological processes are shared between the three single stress responses, we compared their DEGs. Figure $4 a$ shows that there is a large overlap between the DEGs of the single stress responses, ranging from 1716 genes shared between the drought and $P$. rapae sets, to 788 genes between the drought and $B$. cinerea sets, and 777 genes between the $P$. rapae and $B$. cinerea sets. Of all 7173 DEGs (excluding the $1 \mathrm{~d}$ after re-watering time point), 2493 DEGs (35\%) are shared with one or both of the other stresses. A core set of 394 DEGs (5\%) was differentially expressed in response to all three single stresses, and clustered into 12 co-expressed gene clusters (Fig. 4b). Among this core set of shared DEGs are several wellcharacterized hormone-responsive marker genes, including LOX2 (At3g45140), JAZ7 (At2g34600), and JAZ8 (At1g30135) (JA responsive), PDF1.2 (At5g44420) and ORA59 (At1g06160) (JA/ET responsive), PR4 (At3g04720), ERF5 (At5g47230), ERF6 (At4g17490), and ACS2 (At1g01480) (ET responsive), Rap2.6L (At5g13330), and HAI1 (At5g59220) (ABA responsive), and $P R-1$ (At2g14610), PR-5 (At1g75040), and FRK1 (At2g19190) (SA responsive) (Supporting Table S1 for details of their expression patterns). In Fig. $4 c$ the expression patterns of well-characterized marker genes of the response to $B$. cinerea (PDF1.2; At5g44420), P. rapae (LOX2; At3g45140), and drought stress (RAD18; At5g66400) are depicted, confirming that the different stress treatments resulted in the expected response. When looking at the co-expressed gene clusters, only the genes of cluster 1 (top GO terms related to "response to oxygen-containing compound", "response to JA" and "response to wounding"; Supporting Table S3) are regulated in the same direction (activated) during all three individual stress conditions (Fig. 4b). All other gene clusters behave clearly different in response to the three single stresses and are often regulated in opposite directions (Fig. 4b). For example cluster 8 (top GO terms related to "response to other organism", "defense response" and "immune system process") is activated by $B$. cinerea, but repressed by $P$. rapae and drought. Conversely, cluster 10 (top GO terms "multidimensional cell growth", "response to light stimulus", and "cell wall organization") is activated by $P$. rapae, but repressed by $B$. cinerea and drought. The fact that there is an overlap in the expression of genes under all three single stresses, whether in the same or in 
opposite directions, suggests that these genes or their regulators may act as a point of convergence if plants were to experience these stresses in combination.

(a)

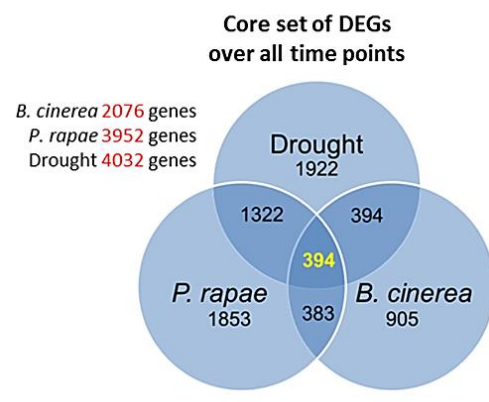

(b)

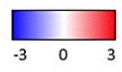

$\mathrm{Bc} \quad \mathrm{Pr} \quad \mathrm{Dr}$

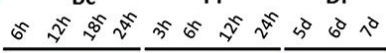

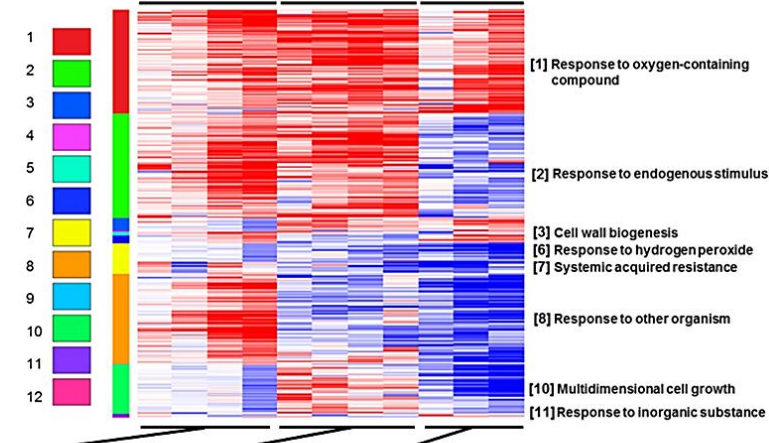

(c)

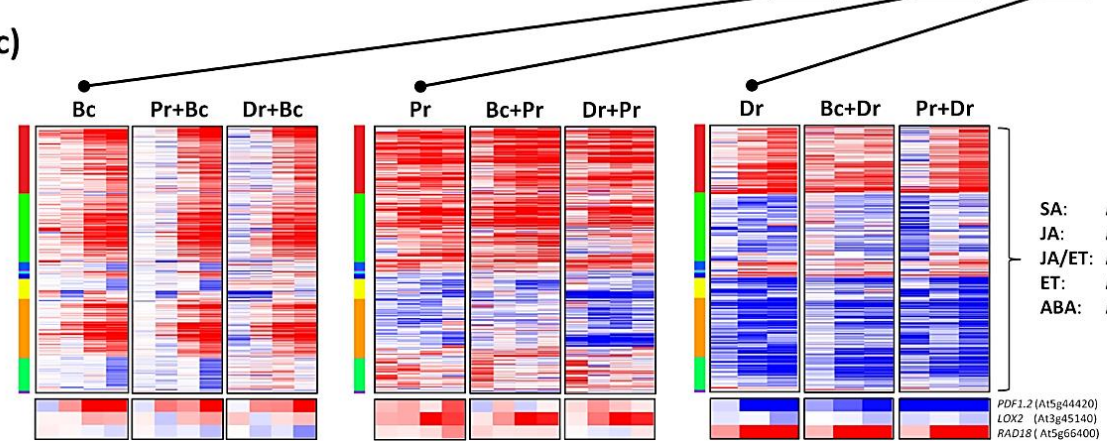

Figure 4. Shared DEGs between the single stress responses. (a) Venn diagram showing the overlap between the DEGs of each of the single stress responses. The total number of unique DEGs per single stress over all time points is shown in red (full data set in Supporting Table S1). (b) Hierarchical clustering of the 394 core DEGs that are shared between the three single stresses (Cosine similarity metric; 12 clusters are color coded in the square boxes on the left). On the right side, the most significant GO term for each cluster (full data set in Supporting Table S3). (c) Comparison of the expression patterns of the 394 core DEGs in response to the single and respective sequential double stresses. Different lanes in (c) reflect the transcription profiles at the time points after treatment as indicated above the lanes in (b). Gene names in the lower right corner represent marker genes of the SA, JA, JA/ET, ET and ABA response pathways that are among the 394 core DEGs. PDF1.2, LOX2, and RAD18, represent known marker genes for the response to $B$. cinerea, $P$. rapae, and drought, respectively. $\mathrm{Bc}$, B. cinerea; $\mathrm{Pr}, \mathrm{P}$. rapae; $\mathrm{Dr}$, drought; $\mathrm{Pr}+\mathrm{Bc}, \mathrm{Dr}+\mathrm{Bc}, \mathrm{Bc}+\mathrm{Pr}, \mathrm{Dr}+\mathrm{Pr}, \mathrm{Bc}+\mathrm{Dr}$, and $\mathrm{Pr}+\mathrm{Dr}$, respective sequential double stresses. Blue-red color key for change in gene expression level: $-3>\log _{2}$ fold change $>3$. 


\section{B. cinerea data set: effect of herbivory and drought stress on dynamics of B. cinerea-induced gene expression}

To investigate the effect of $P$. rapae infestation and drought stress on the dynamics of the transcriptome changes that are induced by $B$. cinerea infection, we analyzed the expression patterns over time of all 2076 B. cinerea-responsive DEGs. Clustering of this group of genes yielded 10 clusters of co-expressed genes across the $B$. cinerea single and sequential stress data sets. Gene clusters that are activated in response to $B$. cinerea infection are enriched for GO terms such as "response to chitin" (Fig. 5, cluster 3; Supporting Table S4), reflecting recognition of fungal chitin by the plant immune system (Pel and Pieterse 2013), and "response to ET stimulus", reflecting the high level of ET emission that is related to plant responses to $B$. cinerea infection (Broekgaarden et al. 2015). In addition, gene clusters that are repressed in response to $B$. cinerea infection are associated with GO terms such as "multidimensional cell growth" (Fig. 5, cluster 9; Supporting Table S4), highlighting the antagonistic relationship between plant growth and defense (Wang and Wang 2014).

In order to identify in greater detail co-regulated genes of which the expression pattern in response to $B$. cinerea infection was affected by either herbivory or drought stress, we used the bioinformatics tool Wigwams (Polanski et al. 2014). The Wigwams algorithm identifies gene modules showing evidence for co-regulation in multiple gene expression time series and identifies signatures of condition-dependent regulatory mechanisms in co-regulated gene sets. Wigwams identified 35 modules of co-regulated genes in the $B$. cinerea data sets (Supporting Fig. S1). Analysis of these clusters for co-expression revealed gene modules of which the expression patters were clearly affected in one or both of the sequential stress treatments in comparison to the $B$. cinerea treatment alone (examples shown in Fig. 6). These gene modules represent signatures of a previous stress in the $B$. cinerea-induced transcriptome profile, and may thus be functionally related to the effect of the first stress on the outcome of the plant response to $B$. cinerea infection. The genes in these Wigwams modules are given in Supporting Table S5 along with their GO term analysis. 


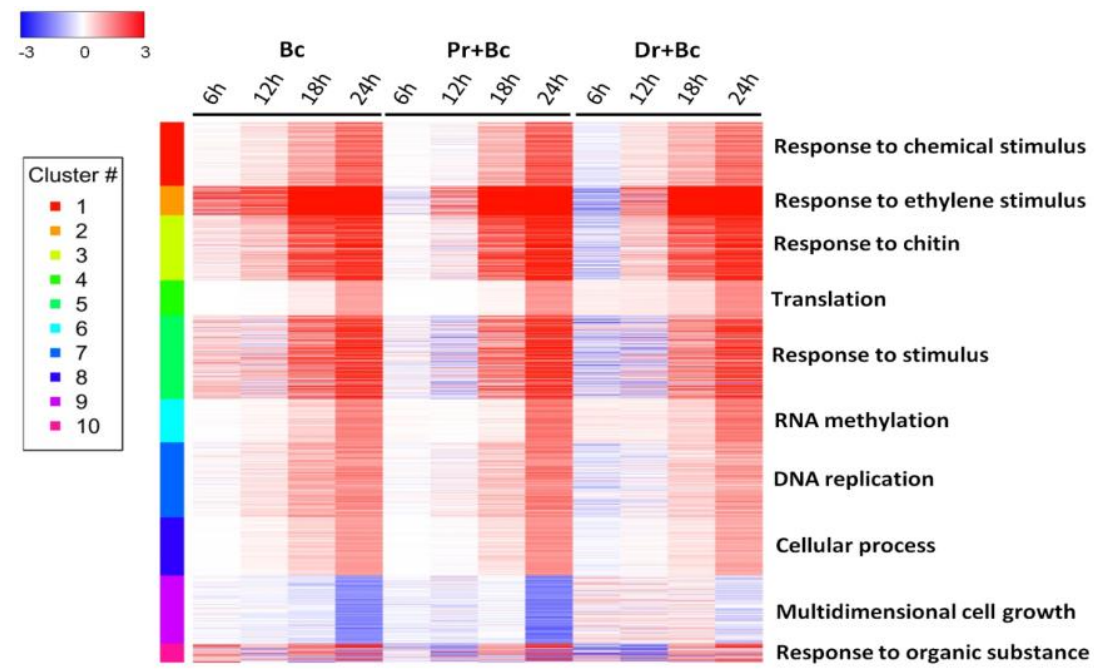

Figure 5. Dynamics of the expression of the $B$. cinerea set of DEGs during single and sequential double stresses. Heatmap showing the expression pattners of the 2076 $B$. cinerea-responsive $\mathrm{DEGs}$ during $B$. cinerea infection on mock pre-treated $(\mathrm{BC}), P$. rapae pre-infested $(\mathrm{Pr}+\mathrm{Bc})$ or drought pre-treated $(\mathrm{Dr}+\mathrm{Bc})$ Arabidopsis plants. The $B$. cinerearesponsive DEGs were clustered using Mclust yielding 10 clusters (colored bars on the left). On the right side, the most significant GO term for each cluster (full data set in Supporting Table S4). Blue-red color key for change in gene expression level: $-3>\log _{2}$ fold change $>$ 3. 


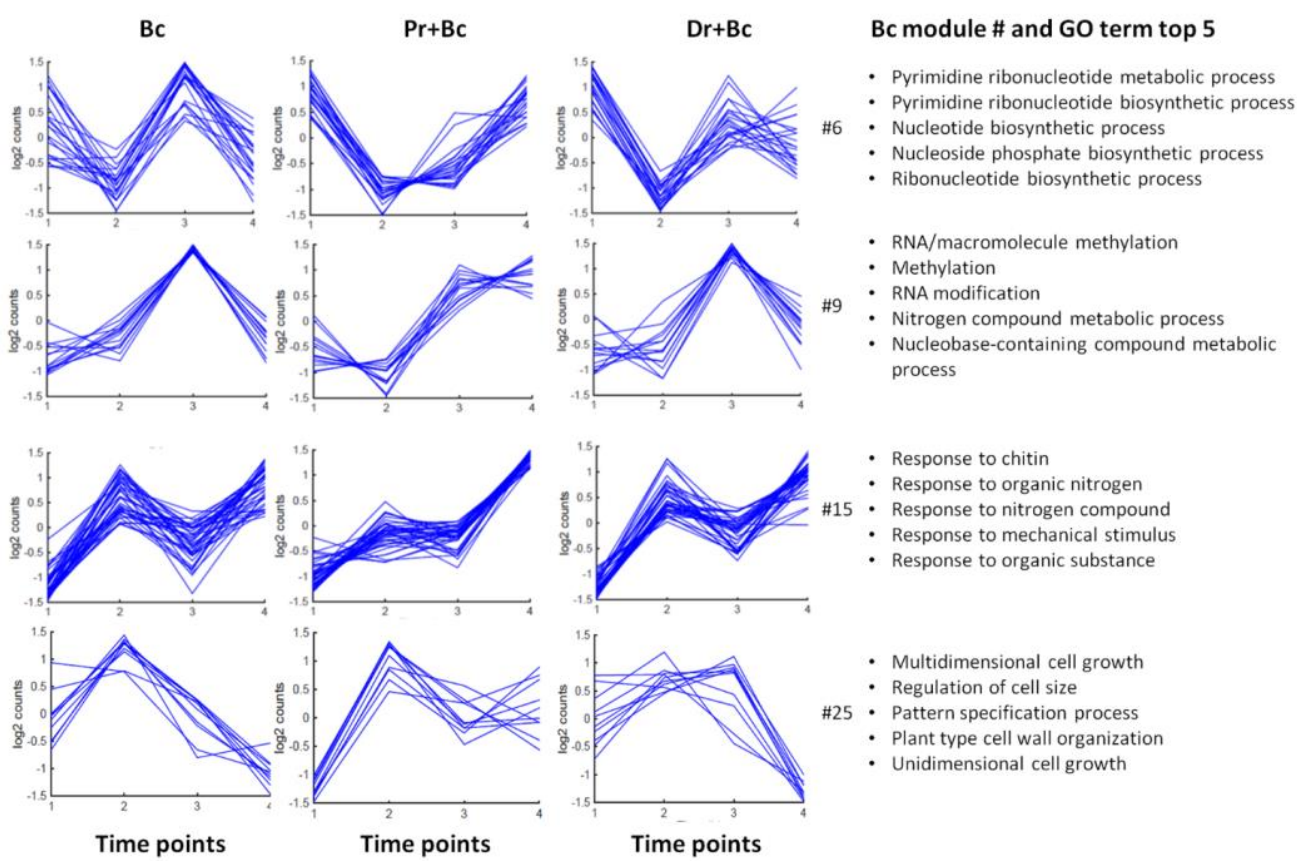

Figure 6. Expression patterns of selected Wigwams modules from the $B$. cinerea set of DEGs during single and sequential stress conditions. A selection of Wigwams modules of co-expressed gene clusters is depicted that show a different pattern in one or both of the sequential stresses $P$. rapae- $B$. cinerea $(\mathrm{Pr}+\mathrm{Bc})$ and drought- $B$. cinerea $(\mathrm{Dr}+\mathrm{Bc})$ in comparison to the single stress $B$. cinerea $(\mathrm{BC})$. The modules represent standardized patterns of differential gene expression over time ( $\log _{2}$ counts). Blue-colored graphs indicate modules of which the genes are significantly co-expressed over time in the given stress condition. Time points $1,2,3$ and 4 represent $6,12,18$ and $24 \mathrm{~h}$ after $B$. cinerea inoculation. The top 5 of GO terms with highest significance in the respective modules are given (full data set for all Wigwams modules is presented in Supporting Table S5).

Among the $B$. cinerea-responsive Wigwams modules of which the coexpression pattern is different when plants were previously exposed to herbivory or drought stress, are gene modules with GO term enrichments for rather general plant processes such as nucleoside biosynthesis and metabolism (modules 6 and 9), and cell growth (module 25), but also modules related to more specific plant processes, such as response to chitin and nitrogen (module 15). Functional analysis of underlying candidate genes should reveal their importance for effects on the outcome of the second stress response.

Further knowledge about the biological processes that are affected when $B$. cinerea infection is preceded by either drought stress or herbivory can be gained by analyzing the phasing of gene expression under the different single and 
sequential stress conditions. We did this by analyzing at which time point a GO term becomes significantly overrepresented in the $B$. cinerea-related DEG set. For this, we clustered all $B$. cinerea-responsive DEGs according to their time point of first differential expression, divided them over activated and repressed genes, and performed GO term analysis on them. Figure 7 shows the timing and strength of the onset of significant GO term enrichment in the single and sequential double stress conditions. In the $B$. cinerea single stress data set, GO terms related to responses to ET, fungus, chitin, SA, and oxygen-containing compound, or to processes such as systemic acquired resistance, respiratory burst, and defense appear early in the activated gene set, reflecting the importance of these processes in the plant response to this necrotrophic pathogen. Prior infestation with $P$. rapae clearly delayed the appearance of these GO terms (become visible at 18 hai in Fig. 7), while pre-treatment with drought stress did not have a dramatic effect on the phasing of the activated genes. For the repressed genes in the $B$. cinerea set of DEGs, pre-infestation with $P$. rapae has clearly only minor effects on the GO term phasing. By contrast, pretreatment with drought stress noticeably affected the phasing of GO terms related to responses to fungus, JA, SA, ABA, chitin, and oxygen-containing compound, and to auxin metabolic process, defense, systemic acquired resistance, and glucosinolate biosynthetic process. Remarkably, biological processes related to hormone action prevail in the $B$. cinerea-responsive processes that are sensitive to modulation by prior exposure to one of the other stresses. 


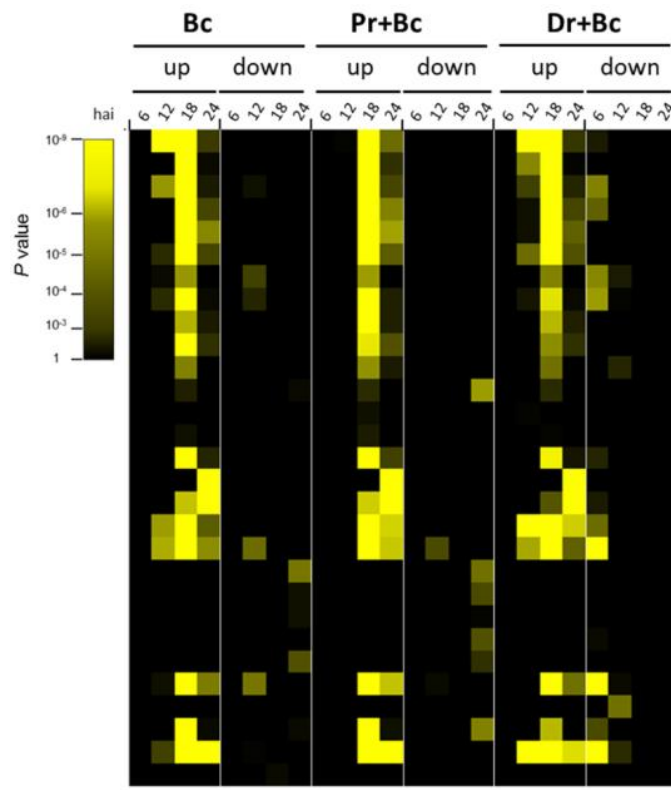

response to ethylene

ethylene biosynthetic process

response to fungus

response to jasmonic acid

response to wounding

respiratory burst

regulation of hydrogen peroxide metabolic process

response to salicylic acid

defense response by callose deposition

response to water deprivation

auxin metabolic process

response to auxin

response to insect

camalexin metabolic process

response to abscisic acid

RNA methylation

cellular macromolecule localization

response to chitin

defense response

cell wall organization or biogenesis

response to gibberellin

cytokinin-activated signaling pathway

response to brassinosteroid

growth

systemic acquired resistance

glucosinolate biosynthetic process

response to lipid

response to oxygen-containing compound photosynthesis

Figure 7. Timing of GO term overrepresentation patterns in $B$. cinerea single and sequential stress data sets. Heatmap represents the strengths of the $P$-values of GO term overrepresentation in the $B$. cinerea-responsive DEG sets (corresponding to the gene lists in Supporting Table S1) that become significantly activated (up) or repressed (down) for the first time at the given stress conditions and time points. Color index represents level of significance ( $P$-values). On the right, overrepresented $\mathrm{GO}$ terms. $\mathrm{Bc}, B$. cinerea; $\mathrm{Pr}$, $P$. rapae; Dr, drought; hai, h after $B$. cinerea infection. 


\section{Effect of herbivory or drought stress on resistance to B. cinerea}

Both herbivory and drought stress imposed a first-stress-signature in the dynamics of the $B$. cinerea-induced transcriptome profiles. Wigwams analysis gained insight into the identity of candidate genes related to these first-stresssignatures (Supporting Fig. S1; Supporting Table S5), whereas analysis of GO term enrichment provided global insight into the biological processes that were affected by the stress pre-treatments (Fig. 7). To investigate whether the two prior stresses affected the resistance level to $B$. cinerea infection we performed disease resistance bioassays. Inoculation of 5-week-old Arabidopsis Col-0 plants with $B$. cinerea resulted in the development of spreading lesions in about $60 \%$ of the inoculated leaves (Fig. 8).

(a)

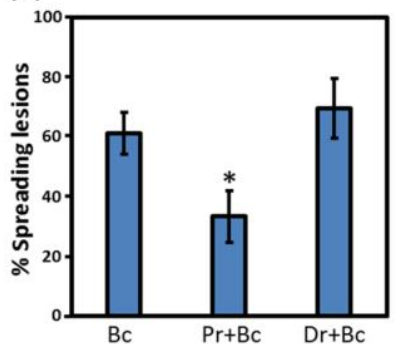

(b)

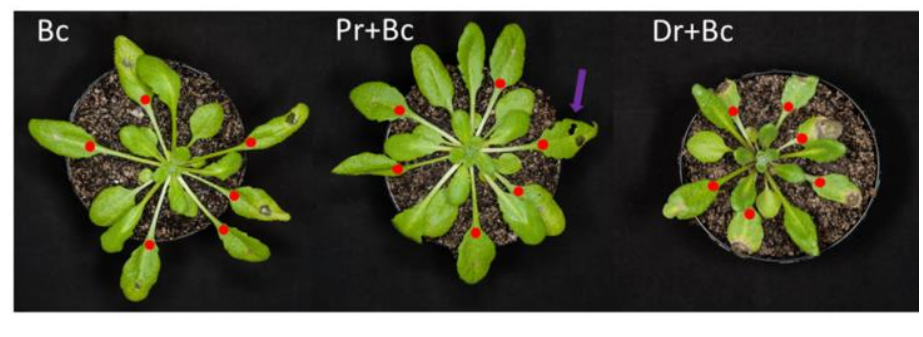

Figure 8. Effect of herbivory and drought stress on resistance of Arabidopsis to $B$. cinerea. (a) Quantification of $B$. cinerea disease symptoms on Arabidopsis accession Col-0 plants (\% spreading lesions per plant). On each plant, six leaves were inoculated with one droplet of $B$. cinerea spores. Three d later, the average number of leaves with spreading lesions was determined per plant. Asterisk indicates statistically significant difference from single stress (Bc) treatment ( $n=6$ plants; Student's $t$-test; $P<0.05)$. (b) Photographs of $B$. cinerea disease symptoms $3 \mathrm{~d}$ after inoculation. $\mathrm{Bc}, B$. cinerea-inoculated plants; $\mathrm{Pr}+\mathrm{Bc}, B$. cinerea-inoculated plants that prior to inoculation were exposed to herbivory by $P$. rapae larvae for $24 \mathrm{~h}$; $\mathrm{Dr}+\mathrm{BC}, \mathrm{B}$. cinerea-inoculated plants that prior to inoculation received a drought treatment for 7 days, followed by a re-watering phase of one day. Red dots, $B$. cinerea-inoculated leaves; purple arrow, damage caused by $P$. rapae feeding.

Plants that were exposed to drought stress prior to $B$. cinerea inoculation showed a similar percentage of leaves with spreading lesions ( $70 \%)$. Interestingly, plants that were exposed to herbivory prior to $B$. cinerea inoculation showed a significantly enhanced level of resistance against $B$. cinerea infection (average $\sim 35 \%$ spreading lesions). Together these results indicate that a first stress can have strong effects on the outcome of the adaptive stress response to a second stress, depending on the nature of the first stress. 


\section{P. rapae data set: effect of $B$. cinerea infection and drought stress on dynamics of $P$. rapae-induced gene expression}

The same approach as described above for the $B$. cinerea transcriptome data was taken to investigate the effect of prior $B$. cinerea infection and drought stress on the transcriptional dynamics that are induced by $P$. rapae feeding. Analysis of the global expression profiles of the $3952 P$. rapae-responsive DEGs yielded 9 clusters of co-expressed genes during single and sequential $P$. rapae stress (Fig. 9).

As expected, $P$. rapae feeding induced many genes related to the GO term "response to JA stimulus" (Fig. 9, cluster 7; Supporting Table S6), reflecting induced defenses that are triggered by herbivory-inflicted wounding (Wasternack 2015). In addition, $P$. rapae feeding repressed SA-related genes associated with GO terms "defense response" and "systemic acquired resistance" (Fig. 9, cluster 3; Supporting Table S6), reflecting the antagonistic relationship between JA- and SA-dependent defenses (Pieterse et al. 2012). In analogy with what we observed in the $B$. cinerea data sets, the general gene expression patterns over time overlapped greatly between the responses to $P$. rapae single and sequential double stress treatments, again suggesting that Arabidopsis is capable of reprogramming its transcriptome to the last stress encountered, thereby overruling the effects of the prior stresses. For instance, while prior drought stress impacted the expression of over 1000 genes in the leaf tissue just prior to the start of the $P$. rapae treatment (Supporting Table S1; Fig. 3 last lane), already from the first time point $(3 \mathrm{~h})$ after herbivory this effect was mostly vanished in the $P$. rapae-induced profiles, which readily followed a similar expression pattern as in the $P$. rapae single treatment (Fig. 9). A similar pattern is visible in the core set of 394 DEGs (Fig. 4c, middle panel). Nevertheless, during the sequential stresses first-stress signatures can be detected, e.g. genes in cluster 3 of Fig. 9 and clusters 7 and 8 of Fig. 4c (middle panel) show a weaker repression in the $B$. cinerea pre-treatment and a stronger repression in the drought pre-treatment. In general, these $P$. rapae-related results confirm previous findings (Davila Olivas et al. 2016). 


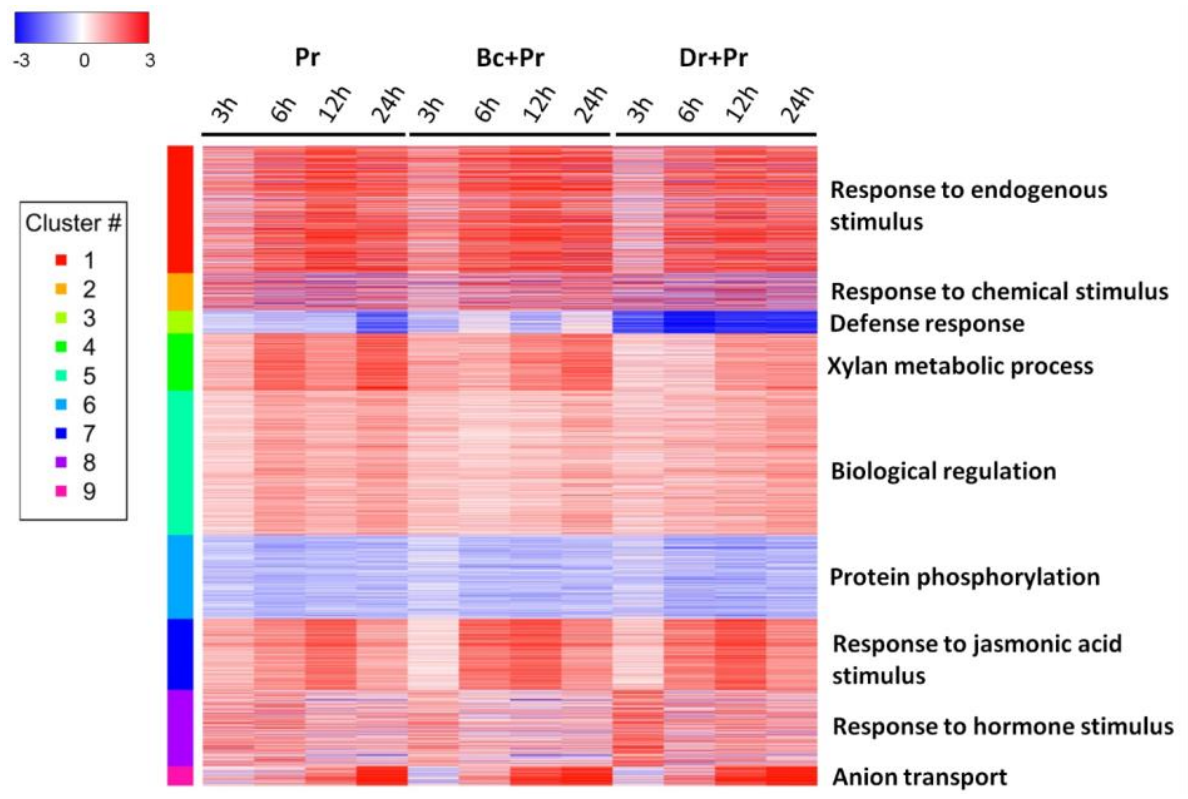

Figure 9. Dynamics of the expression of the $P$. rapae set of DEGs during single and sequential double stresses. Heatmap showing the expression patterns over time of the $3952 P$. rapae-responsive DEGs during feeding of $P$. rapae on control $(\mathrm{Pr}), B$. cinerea pre-infected $(\mathrm{Bc}+\mathrm{Pr})$, or drought pre-treated $(\mathrm{Dr}+\mathrm{Pi})$ Arabidopsis plants. The $P$. rapaeresponsive DEGs were clustered using Mclust yielding 9 clusters (colored bars on the left). On the right side, the most significant GO term for each cluster (full data set in Supporting Table S6). Blue-red color key for change in gene expression level: $-3>\log _{2}$ fold change $>$ 3.

To pinpoint co-regulated genes whose expression pattern in response to herbivory is affected by prior $B$. cinerea infection or drought stress, the set of $P$. rapae-responsive DEGs was analyzed with the Wigwams algorithm. Wigwams identified 93 modules of co-regulated genes in the $P$. rapae set of DEGs. Analysis of these clusters for co-expression under the single and sequential double stress conditions revealed gene modules of which the expression patterns were clearly affected by one or both of the sequential double stress treatments in comparison to the $P$. rapae treatment alone (examples shown in Fig. 10; full set in Supporting Fig. S2). The identities of the genes in the $P$. rapae-related Wigwams gene modules are given in Supporting Table S5 along with their GO term analysis. It is beyond the scope of this paper to discuss the identity of the genes in detail. However, among the $P$. rapae-responsive Wigwams modules of which the co-expression pattern is clearly different when plants were exposed before to $B$. cinerea infection or drought stress, are gene modules with GO term enrichments for xylem, phloem and tissue development 
(module 3), cell wall biosynthesis processes (module 45), and responses to JA and wounding (module 69).
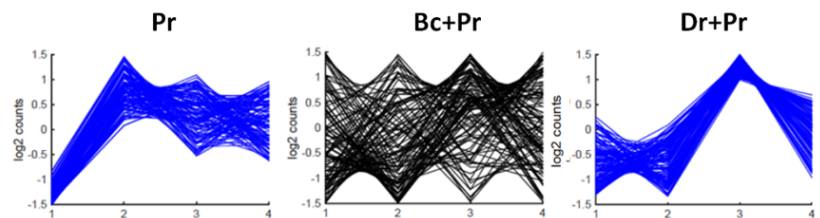

Pr module \# and GO term top 5
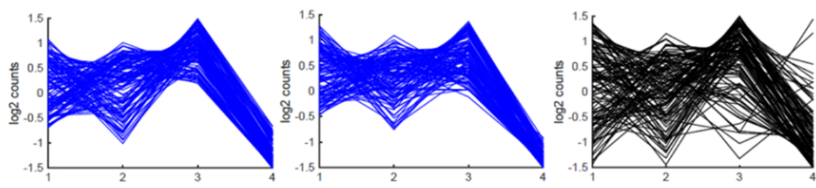

- Xylem development

- Tissue development

- Phloem or xylem histogenesis

- Meristem development

- Anatomic structure arrangement
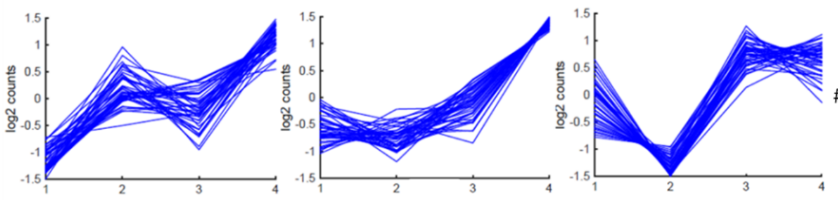

- Cell biogenesis

- Glucoronoxylane metabolic process

- Xylan biosynthesis/metabolic process

\#45 - Cell wall macromolecule biosynthetic process

- Cellular component macromolecule biosynthetic process

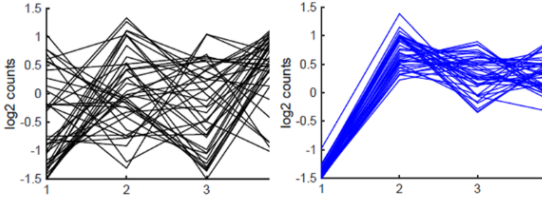

Time points

Time points

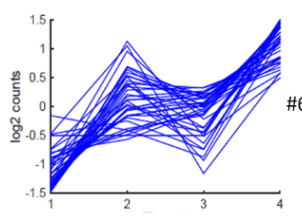

Time points

- Biological regulation

Extracellular region

- Flavonol synthase activity

Figure 10. Expression patterns of selected Wigwams modules from the $P$. rapae set of DEGs during single and sequential stress conditions. A selection of Wigwams modules of co-expressed gene clusters is depicted that show a different pattern in one or both of the sequential stresses $B$. cinerea- $P$. rapae $(\mathrm{Bc}+\mathrm{Pr})$ and drought- $P$. rapae $(\mathrm{Dr}+\mathrm{Pr})$ in comparison to the single stress $P$. rapae $(\mathrm{Pr})$. The modules represent standardized patterns of differential gene expression over time ( $\log _{2}$ counts). Blue-colored graphs indicate modules of which the genes are significantly co-expressed over time in the given stress condition. In the black-colored graphs, the genes in the module are not significantly co-expressed. Time points $1,2,3$ and 4 represent $3,6,12$ and $24 \mathrm{~h}$ after $P$. rapae infestation. The top 5 of GO terms with highest significance in the respective modules are given (full data set for all Wigwams modules is presented in Supporting Table S5).

Also for the $P$. rapae data set, phasing of the regulation of biological processes that are associated with herbivory and influenced by prior $B$. cinerea infection or drought stress was determined. Therefore, the level of significance of overrepresentation of the GO terms for the $P$. rapae set of DEGs at their first time of differential expression was assessed. In the $P$. rapae single stress data set, GO terms related to responses to chitin, wounding, JA, ET, SA, auxin, ABA, water deprivation, osmotic stress, and oxygen-containing compound are already highly enriched at $3 \mathrm{~h}$ after infestation in the activated set of DEGs (Fig. 11), 
reflecting the importance of these processes in the response of Arabidopsis to herbivory. In many cases, pre-infection with $B$. cinerea strengthened the level of overrepresentation of these GO-terms at different time points after $P$. rapae infestation, while pre-treatment with drought stress often weakened them. In the repressed set of DEGs, drought stress clearly enhanced the overrepresentation of $\mathrm{GO}$ terms related to responses to chitin, JA, fungus, $A B A$, $\mathrm{SA}$, bacterium, and oxygen-containing compound, and to processes such as systemic acquired resistance, defense response to fungus, and negative regulation of programmed cell death, while $B$. cinerea infection had no major effect on the phasing of these GO terms. Overall, these data indicate that $B$. cinerea infection and drought treatment prior to $P$. rapae infestation affects the timing of several defense-related processes, in particular responses to JA, ABA, $\mathrm{SA}$ and ET, corroborating the notion that different stresses interact via the hormone-regulated signaling network. 


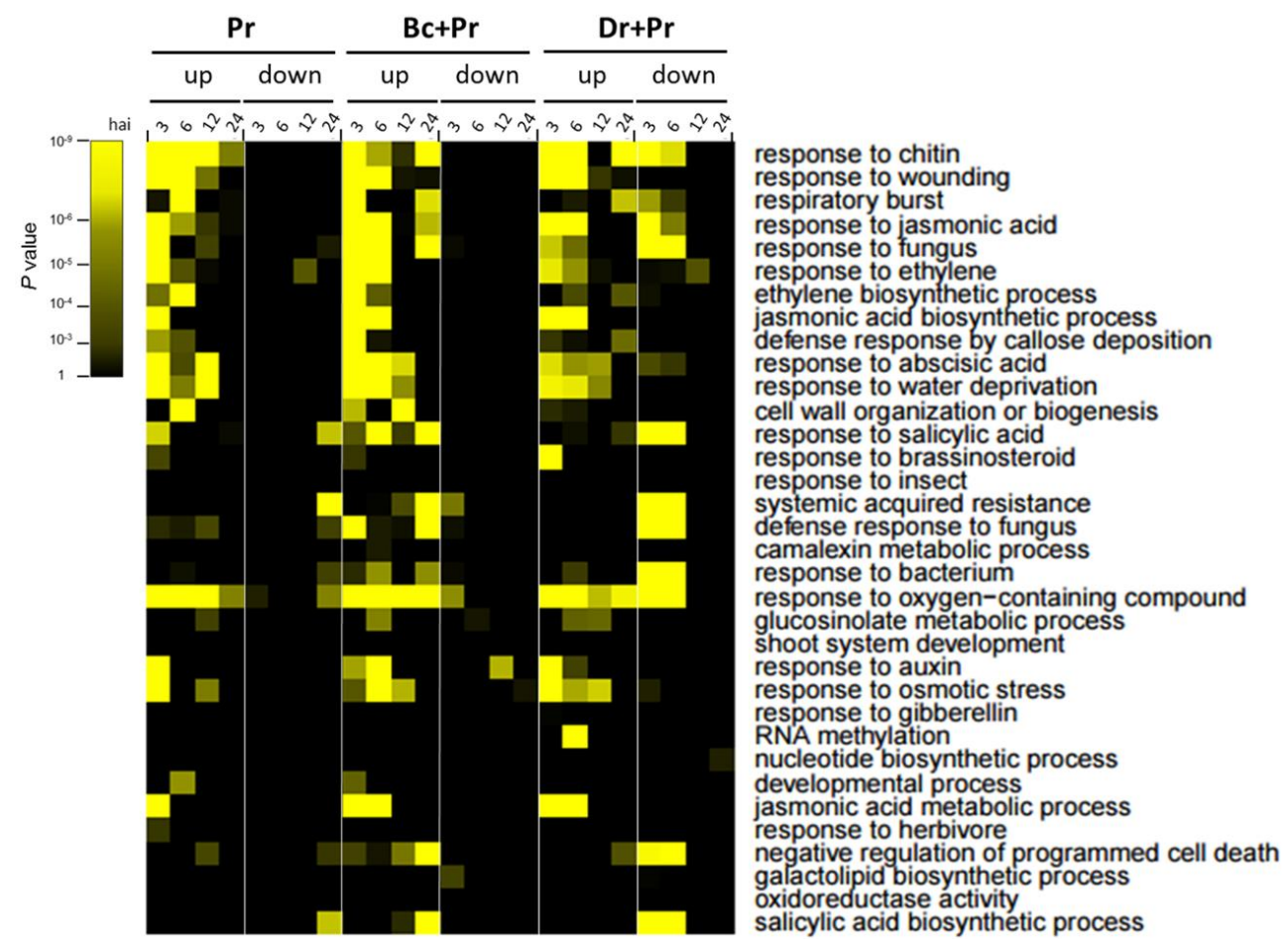

Figure 11. Timing of GO term overrepresentation patterns in $P$. rapae single and sequential double stress data sets. Heatmap represents the strengths of the $P$-values of GO term overrepresentation of $P$. rapae-responsive DEG sets (corresponding to the gene lists in Supporting Table S1) that become significantly activated (up) or repressed (down) for the first time at the given stress conditions and time points. Color index represents level of significance $(P-$ values). On the right, overrepresented GO terms. Bc, $B$. cinerea; $\mathrm{Pr}, P$. rapae; Dr, drought; hai, h after $P$. rapae infestation. 


\section{Drought data set: effect of B. cinerea infection and herbivory on dynamics of drought stress-induced gene expression}

Also for drought stress we investigated the effect of the other two stresses on the dynamics of the transcriptome changes that are induced by this abiotic stress. We analyzed the dynamics of the global expression patterns of the 4032 drought-responsive DEGs during single and sequential stress with drought as the second stress, which yielded 10 clusters of co-expressed genes (Fig. 12). GO term analysis of overrepresented biological processes in each cluster highlights the main differentially regulated biological processes. As expected, drought stress induced a relatively large number of genes related to GO term "response to water deprivation" (Fig. 12, cluster 6; Supporting Table S7) and GO terms related to "response to oxygen-containing compound" (clusters 3 and 10, and cell wall-related processes (cluster 7). Another feature that stands out is the association of drought stress with massive repression of genes, many of which are associated with biological processes such as "photosynthesis" and "defense response" (clusters 1, 2, 5 and 8), reflecting the fact that drought-stressed plants shift their strategy from energy-demanding processes related to growth and immunity to adaptation to the abiotic stress condition. Interestingly, after 1 day of re-watering $(7+1 \mathrm{~d}$ columns in Fig. 12), the drought-induced transcriptional changes that intensified over the 7-d period of water withhold, were for $77 \%$ (3106 of the 4032 DEGs; Supporting Table S1) reset towards basal levels within $24 \mathrm{~h}$, demonstrating the plant's ability to swiftly redirect transcriptional programming when drought stress is relieved. Similar to what we observed for the $B$. cinerea and $P$. rapae sequential double stress responses, the gene expression patterns over time in the sequential drought double stress treatments were to a large extent similar to those inflicted by the single drought treatment. In the core set of 394 DEGs it is clear that on the first time point after the start of the drought treatment $(5 \mathrm{~d}), B$. cinerea and $P$. rapae pretreatment still had noticeable effects on the drought-induced gene expression profiles (Fig. 4c, right panel; compare the left lanes of $\mathrm{Dr}, \mathrm{Bc}+\mathrm{Dr}$, and $\mathrm{Pr}+\mathrm{Dr}$ ). However, at the later time points (6 and 7 d) these effects dampened off and the expression patterns became more similar to that of the drought single treatment. Nevertheless, prior stress caused by $B$. cinerea infection or $P$. rapae infestation left first-stress-signatures in the drought-induced transcriptome. 


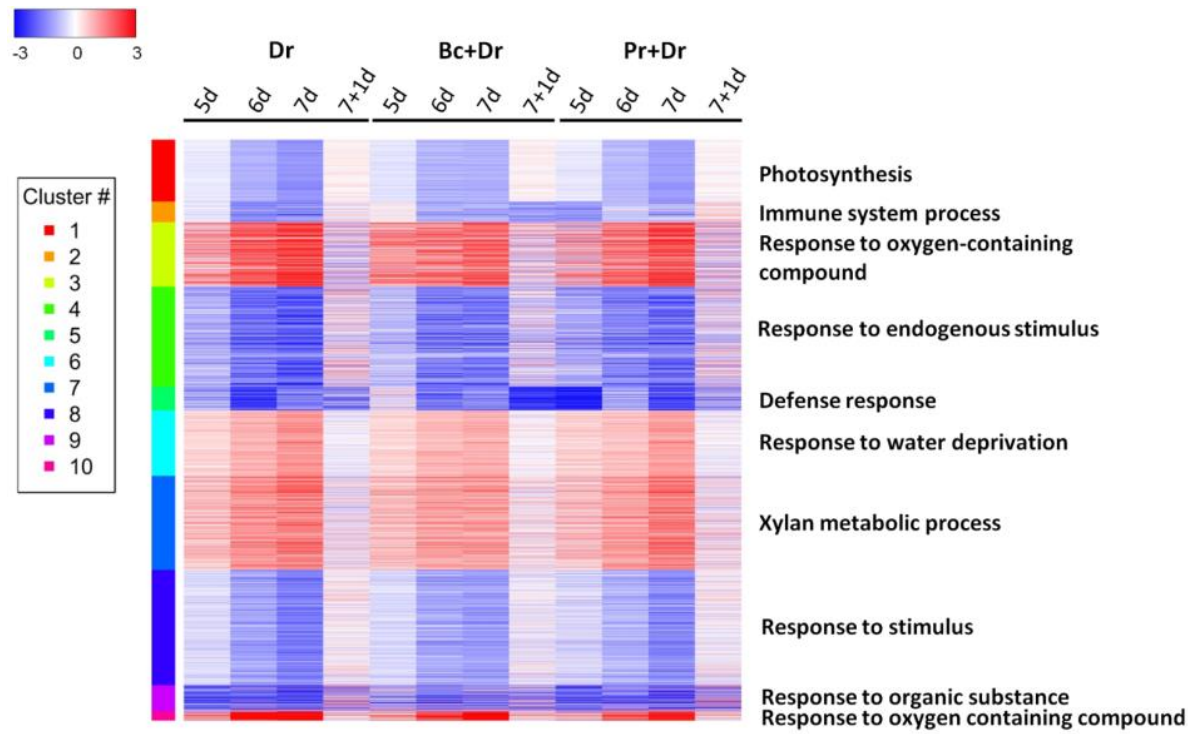

Figure 12. Dynamics of the expression of the drought set of DEGs during single and sequential double stresses. Heatmap showing the expression patterns over time of the 4032 drought-induced DEGs during a 7-d period of water withhold and $1 \mathrm{~d}$ after rewatering on control (Dr), $B$. cinerea pre-infected $(B c+D r)$, or $P$. rapae pre-infested $(\mathrm{Pr}+\mathrm{Dr})$ Arabidopsis plants. The drought-responsive DEGs were clustered using Mclust yielding 10 clusters (colored bars on the left). On the right side, the most significant GO term for each cluster (full data set in Supporting Table S7). Blue-red color key for change in gene expression level: $-3>\log _{2}$ fold change $>3$.

Wigwams analysis of co-regulated genes in the drought data sets identified 72 co-expressed gene modules under the single and sequential double drought stress conditions (examples in Fig. 13; full set in Supporting Fig. S3). The identities of the genes in these Wigwams gene modules are given in Supporting Table S5 along with their GO term analysis. Wigwams modules with clear changes in expression pattern when drought-stressed plants were pre-treated with either $B$. cinerea or $P$. rapae represent genes related to the biological processes such as SA and defense signaling (module 10 and 55), and Nucleosome organisation (module 11). Future analysis of candidate genes in these modules should reveal their importance for the outcome of the combinatorial stress responses. 


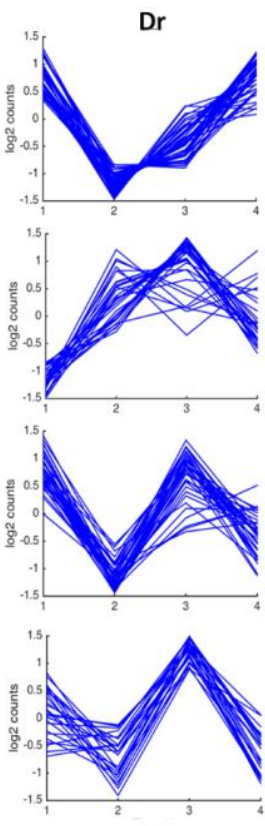

Time points
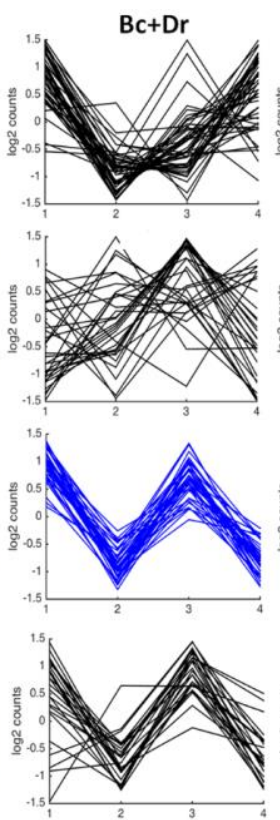

Time points

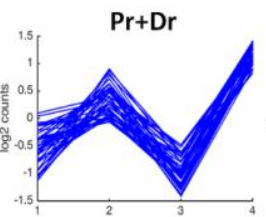

Dr module \# and GO term top 5

- Defense response

- Immune system process

\#10 - Innate immune response

- Immune response

- Salicylic acid biosynthetic process

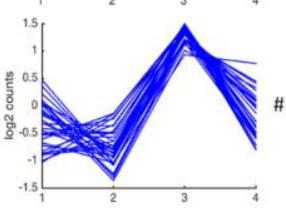

- Nucleosome assembly

- Nucleosome organization

11 Protein-DNA complex assembly

- Protein-DNA complex subunit organization

- Chromatin assembly

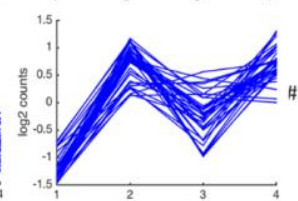

Systemic acquired resistance

Innate immune response Immune response

- Defense response, incompatible interaction

- Immune system process

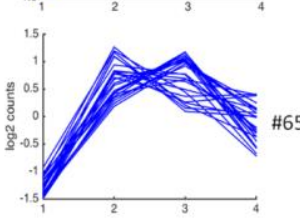

Time points

- Response to chitin

- Response to organic nitrogen

Response to endoplasmic reticulum stress

Response to nitrogen compound

- Response to stress

Figure 13. Expression patterns of selected Wigwams modules from the drought set of DEGs during single and sequential stress conditions. A selection of Wigwams modules of co-expressed gene clusters is depicted that show a different pattern in one or both of the sequential stresses $B$. cinerea-drought $(\mathrm{Bc}+\mathrm{Dr})$ and $P$. rapae-drought $(\mathrm{Pr}+\mathrm{Dr})$ in comparison to the single stress drought ( $\mathrm{Dr}$ ). The modules represent standardized patterns of differential gene expression over time ( $\log _{2}$ counts). Blue-colored graphs indicate modules of which the genes are significantly co-expressed over time in the given stress condition. In the black-colored graphs, the genes in the module are not significantly co-expressed. Time points 1, 2, 3 and 4 represent 5, 6, 7 and 7+1 d after onset of water withhold, in which the $7+1 \mathrm{~d}$ time point represents plants that were re-watered for $1 \mathrm{~d}$ after the 7-d drought period. The top 5 of GO terms with highest significance in the respective modules are given (full data set for all Wigwams modules is presented in Supporting Table S5). 
Also for the drought DEGs, we analyzed the timing and level of significance of overrepresentation of all the GO terms in the single and sequential double stress time series (Fig. 14). In the drought single stress data set, GO terms related to responses to oxidative stress, water deprivation, osmotic stress, ABA, and oxygen-containing compound, and to processes such as phenylpropanoid biosynthesis, cell wall biogenesis, and lignin metabolism are enriched in the activated gene set at the first day of sampling, while responses to wounding and JA follow somewhat later. In the repressed gene set, GO terms related to responses to JA, SA, chitin, fungus, insect and oxygen-containing compound and to processes such as photosynthesis, shoot system development, systemic acquired resistance, glucosinolate metabolic process, nitrogen compound transport, and respiratory burst are enriched already at the first sampling point. This highlights the biological processes that are engaged or affected during drought stress. Interestingly, pre-infection with $B$. cinerea accelerated the phasing of activated genes associated with biological processes such as responses to chitin, wounding, osmotic stress, ABA, and JA. In the repressed gene set, GO terms related to responses to chitin, fungus, and SA, and to systemic acquired resistance became later enriched than in the single stress data set. When plants were pre-infested with $P$. rapae, the phasing of the drought responsive genes is also clearly affected. Many GO terms in the drought activated gene set become more prominently enriched at later time points. Moreover, in the repressed gene sets GO terms related to responses to water deprivation, osmotic stress, wounding, JA, ABA and ET are highly overrepresented at the first time point of sampling, while this is not the case in the single stress treatment. Like in the $B$. cinerea and $P$. rapae data sets, biological processes related to hormone action become relatively often differentially enriched in the sequential double treatments in comparison to the single stress treatment. 


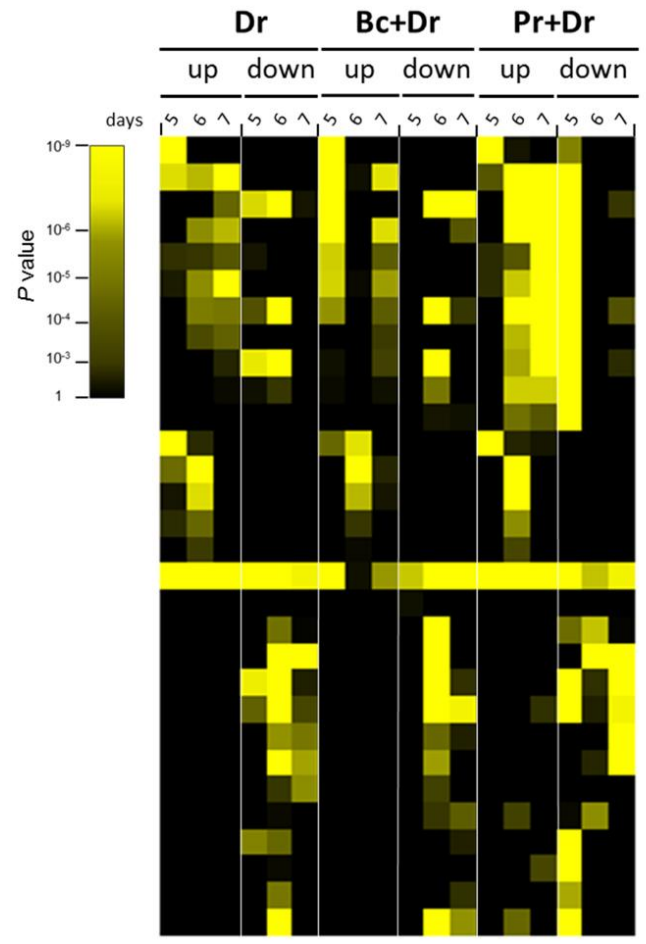

response to oxidative stress

response to water deprivation

response to chitin

response to wounding

response to osmotic stress

response to abscisic acid

response to jasmonic acid

jasmonic acid metabolic process

response to fungus

response to ethylene

ethylene metabolic process

phenylpropanoid biosynthetic process

cell wall biogenesis

cell wall organization or biogenesis

lignin metabolic process

lignin biosynthetic process

response to oxygen-containing compound

response to cytokinin

nitrogen compound metabolic process

photosynthesis

systemic acquired resistance

response to salicylic acid

shoot system development

shoot system morphogenesis

glucosinolate metabolic process

response to auxin

nitrogen compound transport

defense response by callose deposition

response to insect

respiratory burst

Figure 14. Timing of GO term overrepresentation patterns in drought single and sequential stress data sets. Heatmap represents the $P$-values of GO term overrepresentation of drought-responsive DEG sets (corresponding to the gene lists in Supporting Table S1) that become significantly activated (up) or repressed (down) for the first time at the given stress conditions and time points. Color index of $P$-values represents level of significance. On the right, overrepresented $\mathrm{GO}$ terms. $\mathrm{Bc}, B$. cinerea; $\mathrm{Pi}, P$. rapae; Dr, drought; days, d after water withhold.

\section{Effect of stress interactions on plant resistance}

For all three main stresses tested, prior treatment with one of the other stresses imposed a first-stress-signature in the dynamics of their transcriptome profiles. Wigwams analysis provided insight into the identity of the co-expressed genes related to these first-stress-signatures (Figs. 6, 10 and 13), whereas analysis of GO term enrichment at the onset of gene induction provided global insight into the biological processes that were affected during the time course by the prior stress treatment (Figs. 7, 11 and 14). As an example for the effect of prior stress on the level of plant resistance to a second stress, we showed that the level of infection by $B$. cinerea can be significantly altered when plants were pre-disposed to herbivory (Fig. 8). The bioassays with the other sequential stress treatments showed no strong effects of prior stress treatment on the 
performance of the specialist herbivore $P$. rapae (Davila Olivas et al. 2016). In future research, we will functionally analyze candidate genes from the firststress signatures in the second stress profiles to investigate their putative role in a diverse range of multi-stress interactions. 


\section{Discussion}

Plants are often exposed to different abiotic and biotic stresses, which can occur simultaneously or sequentially. How plants selectively adapt their response to this complexity of stresses is largely unknown. In this study, we aimed to gain insight into how plants respond to a biotic or abiotic stress when previously exposed to another stress, using a necrotrophic pathogen, an insect herbivore, and drought as main stress factors. By analyzing the dynamics of the Arabidopsis transcriptome over four consecutive time points we were able to show that 1 ) on average $35 \%$ of the DEGs in a given single stress is also differentially regulated in one or both of the other two single stresses, albeit often in different directions; 2) irrespective of the nature of the first and second stress applied, genes responsive to the second stress rapidly follow a similar pattern as that induced by the second stress alone; 3 ) the Wigwams algorithm identified first-stress-signatures of co-expressed genes that behave differently in the sequential double stress profile in comparison the single stress treatment; 4) plant hormone-related biological processes play a dominant role in the interaction between the studied stress-induced transcriptomes supporting previous findings (Rejeb et al. 2014); and 5) a previous stress can affect the outcome of a response to a sequential second stress, resulting in altered plant resistance.

\section{Transcriptome profiles of different single stress responses show significant overlap}

The expression profiles induced by the single stresses $B$. cinerea infection, $P$. rapae infestation, and drought are clearly different in timing and numbers of activated and repressed genes (Fig. 2). Clustering of the profiles of all DEGs (7355) from the three single stress responses shows that, in general, genes related to stress-related processes are overrepresented in the activated gene sets, while genes related to growth become repressed (Fig. 3). This confirms the notion that plants under stress prioritize appropriate adaptive responses over growth (Vos et al. 2015, Vos et al. 2013a). One third of all DEGs under single stress conditions was also differentially expressed under one or both of the other single stress conditions (Fig. 4a). A set of 394 DEGs was differentially expressed in response to all three single stresses and clustering of their 
expression profiles clearly shows that the expression of the genes in the 12 distinguished clusters is often regulated in opposite directions (up or down), depending on the nature of the stress (Fig. 4b). Only one cluster, containing an overrepresentation of genes related to the GO term "response to oxygencontaining compound" shows a general up-regulation of genes under all three single stress conditions, highlighting that production and responsiveness to oxygen-containing compounds are central to stress responses in general. Overall, these results indicate that a significant proportion of the stress-related transcriptome is engaged by all three stresses tested. The differential stresstype specific up- or down-regulation of genes suggests their positive versus negative function in different adaptive stress responses. When a plant experiences a combination of stresses, this may cause synergistic or antagonistic effects on the level of tolerance to the stresses at hand.

\section{The transcriptome is rewired to the last stress response, but the prior stress leaves first-stress-signatures in the second stress profile}

Analysis of the dynamics of both the single and sequential double stress transcriptome profiles showed that the transcriptome profiles of all possible double stress combinations were remarkably similar to those of the last encountered stress if applied individually (Figs. 4c, 5, 9, and 12). In analogy, $1 \mathrm{~d}$ after re-watering, the drought-induced transcriptome was largely reset to the non-stressed condition (Fig. 12). Apparently, plants are highly plastic in their capacity to adapt to changes in their biotic and abiotic environment, and swiftly rewire their transcriptome to the latest stress encountered. Nevertheless, it has been demonstrated that prior exposure to biotic or abiotic stresses can have dramatic effects on the outcome of the response to a second stress (Rejeb et al. 2014).

To identify genes and biological processes that could contribute to the interaction between different stress responses, different types of analyses have been carried out. When globally inspecting the transcriptional profiles of the single and sequential double stress profiles, it is difficult to pinpoint obvious effects of a first stress on the dynamics of the transcriptional response to a 
second stress. Using Mclust clustering of the transcriptional profiles of the $B$. cinerea, P. rapae and drought sets of DEGs (Figs. 5, 9 and 12) different gene clusters were identified that showed distinct behavior over time during the single and sequential double stress responses. However, in this way relatively small differences between the single and sequential stresses were detected. In order to better pinpoint co-expressed gene clusters that represent first-stresssignatures in the second stress transcriptome profiles, we used the bioinformatics tool Wigwams (Polanski et al. 2014). We were able to dissect the transcriptional profiles into modules of co-regulated genes in time across different conditions. This approach highlighted modules whose gene expression patterns differed from the single stress profile in one or both of the sequential double stress profiles (Figs. 6, 10, and 13, and Supporting Figs. S1, S2 and S3). Future studies should reveal the role of candidate genes in these clusters in shaping the outcome of the adaptive stress responses in the sequential dual stress conditions. Moreover, the Wigwams modules of co-regulated genes could aid in dissecting the regulatory circuitry underlying plant responses to combinatorial stresses, e.g. by analyzing the representation of transcription factors binding motifs in the promoters of the gene modules.

\section{Hormone-related responses prevail in biological processes that are differentially enriched in the double stress transcriptional profiles}

Zooming in on the biological processes that are differentially enriched among the transcriptional profiles of the single versus the sequential double stresses, we monitored GO term enrichment at the time points of first differential expression of all genes. Plots of all biological GO terms that become significantly represented in the set of DEGs at the different time points provide a landscape of the timing at which these biological processes significantly change (Figs. 7, 11 and 14). Interestingly, among all biological processes that become clearly more enriched in the sequential double stresses over their respective single stresses (either in activated or repressed DEGs) are GO terms related to the response to the stress-related hormones JA, ABA, SA, and ET and occasionally to auxin. This observation suggests that responses to these hormones are likely to play a central role in the interaction between the signaling pathways that regulate the 
adaptive responses to the sequential double stresses. In the past, JA, ABA, ET, and SA have been demonstrated to be crucial positive or negative regulators of plant resistance against $B$. cinerea (JA, ET, and SA; El Oirdi et al. 2011, Thomma et al. 1998, Thomma et al. 1999, Vos et al. 2015), P. rapae (JA and ABA; Bodenhausen and Reymond 2007, De Vos et al. 2006, Vos et al. 2013b), and drought stress (ABA; Yamaguchi-Shinozaki and Shinozaki 2006). Hence, interactions between the different hormone-controlled signaling pathways may be decisive in the outcome of the adaptive response when two stresses are encountered sequentially.

\section{Effect of prior stress on level of resistance to subsequent stress}

Classic examples of interactions between defense pathways are the different forms of induced resistance that are triggered by pathogens, insect herbivores and beneficial microbes as they all change the outcome of the defense response against a subsequent invasion by another pathogen or insect in a positive or negative manner (De Vos et al. 2006, Howe and Jander 2008, Pieterse et al. 2014, Poelman et al. 2008, Van Oosten et al. 2008, Vos et al. 2015). Also for abiotic stresses effects on the level of resistance against other abiotic and biotic stresses are documented (Fujita et al. 2006, Rejeb et al. 2014). For the combinations of sequential stresses that were investigated here, we found that prior infestation by $P$. rapae caterpillars changed the level of resistance against $B$. cinerea (Fig. 8), even though the global transcriptional profiles induced by $B$. cinerea as single or second stress did not differ dramatically (Fig. 5).

It can thus be concluded that subtle first-stress-signatures in the double stress transcriptional profile may have significant effects on the outcome of the adaptive response to the second stress, although it cannot be excluded that non-transcriptional changes may also contribute to changes in the level of resistance against the second stress. Previously, it was shown that herbivory on Arabidopsis by $P$. rapae results in a systemic increase in the levels of $\mathrm{JA}$, and that this can prime systemic tissues for enhanced JA-dependent anti-herbivory defenses (Vos et al. 2013b). Since JA-regulated defenses play a major role in resistance against the necrotrophic pathogen $B$. cinerea as well, herbivory-induced priming of $\mathrm{JA}$ responsiveness may contribute to the enhanced resistance level against $B$. cinerea (Fig. 7; Fig. 8).

This study was aimed at analyzing the dynamics of gene expression patterns in response to a set of single and sequential double stresses. Future research will be focused on biological validation of candidate genes in the Wigwams modules with putative major 
roles in shaping the outcome of sequential double stresses. Knowledge on how plants cope with different stresses simultaneously or in sequence will aid in breeding for multi-stress tolerant crops. 


\section{Acknowledgements}

We are thankful to Kim Vermeer, Michel Arts, Elena Kapsomenou, Benno Augustinus, Robert Veldman, and Tom Van den Beuken, who helped with collecting the samples, and Raymond Hulzink and Roy Gorkink (KeyGene) for their skilled execution of the RNA sequencing work and technical discussions. This work was supported by the Netherlands Organization for Scientific Research (NWO) through the Dutch Technology Foundation (STW) STW Perspective Programme 'Learning from Nature' [STW10988], STW VIDI Grant no. 11281 (to SCMvW), STW VENI Grant no. 13682 (to RH), FP7-PEOPLE-2012-IEF no. 327282 (to SP), and ERC Advanced Grant no. 269072 of the European Research Council (to CMJP). 


\section{References}

Agrawal, A.A. (2011) Current trends in the evolutionary ecology of plant defence. Functional Ecology, 25, 420-432.

Anders, S., Pyl, P. and Huber, W. (2014) HTSeq-a Python framework to work with high-throughput sequencing data. Bioinformatics, 31, 166-169.

Appel, H.M., Maqbool, S.B., Raina, S., Jagadeeswaran, G., Acharya, B.R., Hanley, J.C., Miller, K.P., Hearnes, L., Jones, A.D., Raina, R. and Schultz, J.C. (2014) Transcriptional and metabolic signatures of Arabidopsis responses to chewing damage by an insect herbivore and bacterial infection and the consequences of their interaction. Frontiers in Plant Science, 5, 441.

Atkinson, N.J., Lilley, C.J. and Urwin, P.E. (2013) Identification of genes involved in the response of Arabidopsis to simultaneous biotic and abiotic stresses. Plant Physiology, 162, 2028-2041.

Atkinson, N.J. and Urwin, P.E. (2012) The interaction of plant biotic and abiotic stresses: from genes to the field. Journal of Experimental Botany, 63, 3523-3543.

Birkenbihl, R.P., Diezel, C. and Somssich, I.E. (2012) Arabidopsis WRKY33 is a key transcriptional regulator of hormonal and metabolic responses toward Botrytis cinerea infection. Plant Physiology, 159, 266-285.

Bodenhausen, N. and Reymond, P. (2007) Signaling pathways controlling induced resistance to insect herbivores in Arabidopsis. Molecular Plant-Microbe Interactions, 20, 1406-1420.

Boyle, E.I., Weng, S.A., Gollub, J., Jin, H., Botstein, D., Cherry, J.M. and Sherlock, G. (2004) GO: :TermFinder - open source software for accessing Gene Ontology information and finding significantly enriched Gene Ontology terms associated with a list of genes. Bioinformatics, 20, 3710-3715.

Bray, E.A. (1997) Plant responses to water deficit. Trends in Plant Science, 2, 48-

Broekgaarden, C., Caarls, L., Vos, I.A., Pieterse, C.M.J. and Van Wees, S.C.M. (2015) Ethylene:traffic controller on hormonal crossroads to defense. Plant Physiology, 169, 2371-2379.

Caarls, L., Pieterse, C.M.J. and Van Wees, S.C.M. (2015) How salicylic acid takes transcriptional control over jasmonic acid signaling. Frontiers in Plant Science, 6.

Clauw, P., Coppens, F., De Beuf, K., Dhondt, S., Van Daele, T., Maleux, K., Storme, V., Clement, L., Gonzalez, N. and Inzé, D. (2015) Leaf responses to mild drought stress in natural variants of Arabidopsis. Plant Physiology, 167, 800-816.

Cyr, H. and Pace, M.L. (1993) Magnitude and patterns of herbivory in aquatic and terrestrial ecosystems. Nature, 361, 148-150.

Davila Olivas, N.H., Coolen, S., Huang, P., Severing, E., Van Verk, M.C., Hickman, R., Wittenberg, A.H.J., De Vos, M., Prins, M., Van Loon, J.J.A., Aarts, M.G.M., Van Wees, S.C.M., Pieterse, C.M.J. and Dicke, M. (2016) Effect of prior drought and pathogen stress on Arabidopsis transcriptome changes to caterpillar herbivory New Phytologist, doi: 10.1111/nph.13847.

De Vos, M., Van Oosten, V.R., Van Poecke, R.M.P., Van Pelt, J.A., Pozo, M.J., Mueller, M.J., Buchala, A.J., Métraux, J.P., Van Loon, L.C., Dicke, M. and Pieterse, C.M.J. (2005) Signal signature and transcriptome changes of Arabidopsis during pathogen and insect attack. Molecular Plant-Microbe Interactions, 18, 923-937.

De Vos, M., Van Zaanen, W., Koornneef, A., Korzelius, J.P., Dicke, M., Van Loon, L.C. and Pieterse, C.M.J. (2006) Herbivore-induced resistance against microbial pathogens in Arabidopsis. Plant Physiology, 142, 352-363.

Dean, R., Van Kan, J.A.L., Pretorius, Z.A., Hammond-Kosack, K.E., Di Pietro, A., Spanu, P.D., Rudd, J.J., Dickman, M., Kahmann, R., Ellis, J. and Foster, G.D. (2012) The Top 10 fungal pathogens in molecular plant pathology. Molecular Plant Pathology, 13, 414-430.

Diaz, J., Ten Have, A. and Van Kan, J.A.L. (2002) The role of ethylene and wound signaling in resistance of tomato to Botrytis cinerea. Plant Physiology, 129, 13411351. 
El Oirdi, M., Abd El Rahman, T., Rigano, L., El Hadrami, A., Rodriguez, M.C., Daayf, F., Vojnov, A. and Bouarab, K. (2011) Botrytis cinerea manipulates the antagonistic effects between immune pathways to promote disease development in tomato. the Plant Cell, 23, 2405-2421.

Ferrari, S., Galletti, R., Denoux, C., De Lorenzo, G., Ausubel, F.M. and Dewdney, J. (2007) Resistance to Botrytis cinerea induced in Arabidopsis by elicitors is independent of salicylic acid, ethylene, or jasmonate signaling but requires PHYTOALEXIN DEFICIENT3. Plant Physiology, 144, 367-379.

Fraley, C., Raftery, A.E. and Scrucca, L. (2012) Mclust version 4 for R: normal mixture modeling and model-based clustering, classification, and density estimation: Department of Statistics, University of Washington, Washington.

Fujita, M., Fujita, Y., Noutoshi, Y., Takahashi, F., Narusaka, Y., YamaguchiShinozaki, K. and Shinozaki, K. (2006) Crosstalk between abiotic and biotic stress responses: a current view from the points of convergence in the stress signaling networks. Current Opinion in Plant Biology, 9, 436-442.

Geraats, B.P.J., Bakker, P.A.H.M. and Van Loon, L.C. (2002) Ethylene insensitivity impairs resistance to soilborne pathogens in tobacco and Arabidopsis thaliana. Molecular Plant-Microbe Interactions, 15, 1078-1085.

Giron, D., Frago, E., Glevarec, G., Pieterse, C.M.J. and Dicke, M. (2013) Cytokinins as key regulators in plant-microbe-insect interactions: connecting plant growth and defence. Functional Ecology, 27, 599-609.

Glazebrook, J. (2005) Contrasting mechanisms of defense against biotrophic and necrotrophic pathogens. Annual Review of Phytopathology, 43, 205-227.

Hopkins, R.J., Van Dam, N.M. and Van Loon, J.J.A. (2009) Role of glucosinolates in insect-plant relationships and multitrophic interactions. Annual Review of Entomology, 54, 57-83.

Howe, G.A. and Jander, G. (2008) Plant immunity to insect herbivores. Annual Review of Plant Biology, 59, 41-66.

Huang, D.Q., Wu, W.R., Abrams, S.R. and Cutler, A.J. (2008) The relationship of drought-related gene expression in Arabidopsis thaliana to hormonal and environmental factors. Journal of Experimental Botany, 59, 2991-3007.

Ingram, J. and Bartels, D. (1996) The molecular basis of dehydration tolerance in plants. Annual Review of Plant Physiology and Plant Molecular Biology, 47, 377-403.

Johnson, M.T.J. (2011) Evolutionary ecology of plant defences against herbivores. Functional Ecology, 25, 305-311.

Kazan, K. and Lyons, R. (2014) Intervention of phytohormone pathways by pathogen effectors. the Plant Cell, 26, 2285-2309.

Kissoudis, C., Van de Wiel, C., Visser, R.G.F. and Van der Linden, G. (2014) Enhancing crop resilience to combined abiotic and biotic stress through the dissection of physiological and molecular crosstalk. Frontiers in Plant Science, 5, 207.

Koornneef, A., Leon-Reyes, A., Ritsema, T., Verhage, A., Den Otter, F.C., Van Loon, L.C. and Pieterse, C.M.J. (2008) Kinetics of salicylate-mediated suppression of jasmonate signaling reveal a role for redox modulation. Plant Physiology, 147, 13581368.

Laluk, K. and Mengiste, T. (2010) Necrotroph attacks on plants: wanton destruction or covert extortion? The Arabidopsis Book, 8, e0136.

Liu, S., Kracher, B., Ziegler, J., Birkenbihl, R.P. and Somssich, I.E. (2015) Negative regulation of ABA signaling by WRKY33 is critical for Arabidopsis immunity towards Botrytis cinerea 2100. eLife, 4, e07295.

Love, M.I., Huber, W. and Anders, S. (2014) Moderated estimation of fold change and dispersion for RNA-seq data with DESeq2. Genome Biology, 15.

Mohr, P.G. and Cahill, D.M. (2003) Abscisic acid influences the susceptibility of Arabidopsis thaliana to Pseudomonas syringae pv. tomato and Peronospora parasitica. Functional Plant Biology, 30, 461-469.

O'Brien, J.A. and Benková, E. (2013) Cytokinin cross-talking during biotic and abiotic stress responses. Frontiers in Plant Science, 4, 451. 
Pel, M.J.C. and Pieterse, C.M.J. (2013) Microbial recognition and evasion of host immunity. Journal of Experimental Botany, 64, 1237-1248.

Pieterse, C.M.J., Leon-Reyes, A., Van der Ent, S. and Van Wees, S.C.M. (2009) Networking by small-molecule hormones in plant immunity. Nature Chemical Biology, 5, 308-316.

Pieterse, C.M.J., Van der Does, D., Zamioudis, C., Leon-Reyes, A. and Van Wees, S.C.M. (2012) Hormonal modulation of plant immunity. Annual Review of Cell and Developmental Biology, 28, 489-521.

Pieterse, C.M.J., Zamioudis, C., Berendsen, R.L., Weller, D.M., Van Wees, S.C.M. and Bakker, P.A.H.M. (2014) Induced systemic resistance by beneficial microbes. Annual Review of Phytopathology, 52, 347-375.

Poelman, E.H., Broekgaarden, C., Van Loon, J.J.A. and Dicke, M. (2008) Early season herbivore differentially affects plant defence responses to subsequently colonizing herbivores and their abundance in the field. Molecular Ecology, 17, 3352-3365.

Polanski, K., Rhodes, J., Hill, C., Zhang, P.J., Jenkins, D.J., Kiddle, S.J., Jironkin, A., Beynon, J., Buchanan-Wollaston, V., Ott, S. and Denby, K.J. (2014) Wigwams: identifying gene modules co-regulated across multiple biological conditions. Bioinformatics, 30, 962-970.

Prasch, C.M. and Sonnewald, U. (2013) Simultaneous application of heat, drought, and virus to Arabidopsis plants reveals significant shifts in signaling networks. Plant Physiology, 162, 1849-1866.

Ramegowda, V. and Senthil-Kumar, M. (2015) The interactive effects of simultaneous biotic and abiotic stresses on plants: Mechanistic understanding from drought and pathogen combination. Journal of Plant Physiology, 176, 47-54.

Rasmussen, S., Barah, P., Suarez-Rodriguez, M.C., Bressendorff, S., Friis, P., Costantino, P., Bones, A.M., Nielsen, H.B. and Mundy, J. (2013) Transcriptome responses to combinations of stresses in Arabidopsis. Plant Physiology, 161, 1783-1794.

Rehrig, E.M., Appel, H.M., Jones, A.D. and Schultz, J.C. (2014) Roles for jasmonate- and ethylene-induced transcription factors in the ability of Arabidopsis to respond differentially to damage caused by two insect herbivores. Frontiers in Plant Science, 5, 407.

Rejeb, I.B., Pastor, V. and Mauch-Mani, B. (2014) Plant responses to simultaneous biotic and abiotic stress: molecular mechanisms. Plants, 3, 458-475.

Reymond, P., Bodenhausen, N., Van Poecke, R.M.P., Krishnamurthy, V., Dicke, M. and Farmer, E.E. (2004) A conserved transcriptional pattern in response to a specialist and a generalist herbivore. the Plant Cell, 16, 3132-3147.

Reymond, P. and Farmer, E.E. (1998) Jasmonate and salicylate as global signals for defense gene expression. Current Opinion in Plant Biology, 1, 404-411.

Reymond, P., Weber, H., Damond, M. and Farmer, E.E. (2000) Differential gene expression in response to mechanical wounding and insect feeding in Arabidopsis. The Plant Cell, 12, 707-719.

Riera, M., Valon, C., Fenzi, F., Giraudat, J. and Leung, J. (2005) The genetics of adaptive responses to drought stress: abscisic acid-dependent and abscisic acidindependent signalling components. Physiologia Plantarum, 123, 111-119.

Rivero, R.M., Mestre, T.C., Mittler, R., Rubio, F., Garcia-Sanchez, F. and Martinez, V. (2014) The combined effect of salinity and heat reveals a specific physiological, biochemical and molecular response in tomato plants. Plant Cell and Environment, 37, 1059-1073.

Robert-Seilaniantz, A., Grant, M. and Jones, J.D.G. (2011) Hormone crosstalk in plant disease and defense: more than just jasmonate-salicylate antagonism. Annual Review of Phytopathology, 49, 317-343.

Rowe, H.C., Walley, J.W., Corwin, J., Chan, E.K.F., Dehesh, K. and Kliebenstein, D.J. (2010) Deficiencies in jasmonate-mediated plant defense reveal quantitative variation in Botrytis cinerea pathogenesis. PLoS Pathogens, 6, e1000861.

Santino, A., Taurino, M., De Domenico, S., Bonsegna, S., Poltronieri, P., Pastor, V. and Flors, V. (2013) Jasmonate signaling in plant development and defense response to multiple (a)biotic stresses. Plant Cell Reports, 32, 1085-1098. 
Schachtman, D.P. and Goodger, J.Q.D. (2008) Chemical root to shoot signaling under drought. Trends in Plant Science, 13, 281-287.

Sewelam, N., Oshima, Y., Mitsuda, N. and Ohme-Takagi, M. (2014) A step towards understanding plant responses to multiple environmental stresses: a genomewide study. Plant Cell and Environment, 37, 2024-2035.

Sham, A., Moustafa, K., Al-Ameri, S., Al-Azzawi, A., Iratni, R. and AbuQamar, S. (2015) Identification of Arabidopsis candidate genes in response to biotic and abiotic stresses using comparative microarrays. PLOS ONE, 10, e0125666.

Shinozaki, K., Yamaguchi-Shinozaki, K. and Seki, M. (2003) Regulatory network of gene expression in the drought and cold stress responses. Current Opinion in Plant Biology, 6, 410-417.

Soler, R., Erb, M. and Kaplan, I. (2013) Long distance root-shoot signalling in plant-insect community interactions. Trends in Plant Science, 18, 149-156.

Staats, M. and Van Kan, J.A.L. (2012) Genome update of Botrytis cinerea strains B05.10 and T4. Eukaryotic Cell, 11, 1413-1414.

Stam, J.M., Kroes, A., Li, Y.H., Gols, R., Van Loon, J.J.A., Poelman, E.H. and Dicke, M. (2014) Plant interactions with multiple insect herbivores: from community to genes. Annual Review of Plant Biology, 65, 689-713.

Suzuki, N., Rivero, R.M., Shulaev, V., Blumwald, E. and Mittler, R. (2014) Abiotic and biotic stress combinations. New Phytologist, 203, 32-43.

Thomma, B.P.H.J., Eggermont, K., Penninckx, I.A.M.A., Mauch-Mani, B., Vogelsang, R., Cammue, B.P.A. and Broekaert, W.F. (1998) Separate jasmonatedependent and salicylate-dependent defense-response pathways in Arabidopsis are essential for resistance to distinct microbial pathogens. Proceedings of the National Academy of Sciences of the United States of America, 95, 15107-15111.

Thomma, B.P.H.J., Eggermont, K., Tierens, K.F.M. and Broekaert, W.F. (1999) Requirement of functional ethylene-insensitive 2 gene for efficient resistance of Arabidopsis to infection by Botrytis cinerea. Plant Physiology, 121, 1093-1102.

Trapnell, C., Pachter, L. and Salzberg, S.L. (2009) TopHat: discovering splice junctions with RNA-Seq. Bioinformatics, 25, 1105-1111.

Van Kan, J.A.L. (2006) Licensed to kill: the lifestyle of a necrotrophic plant pathogen. Trends in Plant Science, 11, 247-253.

Van Oosten, V.R., Bodenhausen, N., Reymond, P., Van Pelt, J.A., Van Loon, L.C., Dicke, M. and Pieterse, C.M.J. (2008) Differential effectiveness of microbially induced resistance against herbivorous insects in Arabidopsis. Molecular Plant-Microbe Interactions, 21, 919-930.

Van Verk, M.C., Hickman, R., Pieterse, C.M.J. and Van Wees, S.C.M. (2013) RNA-Seq: revelation of the messengers. Trends in Plant Science, 18, 175-179.

Van Wees, S.C.M., Van Pelt, J.A., Bakker, P.A.H.M. and Pieterse, C.M.J. (2013) Bioassays for assessing jasmonate-dependent defenses triggered by pathogens, herbivorous Insects, or beneficial rhizobacteria. Methods in Molecular Biology, 1011, 3549.

Verhage, A., Vlaardingerbroek, I., Raaymakers, C., Van Dam, N., Dicke, M., Van Wees, S.C.M. and Pieterse, C.M.J. (2011) Rewiring of the jasmonate signaling pathway in Arabidopsis during insect herbivory. Frontiers in Plant Science, 2, 47.

Vos, I.A., Moritz, L., Pieterse, C.M.J. and Van Wees, S.C.M. (2015) Impact of hormonal crosstalk on plant resistance and fitness under multi-attacker conditions. Frontiers in Plant Science, 6, 639.

Vos, I.A., Pieterse, C.M.J. and Van Wees, S.C.M. (2013a) Costs and benefits of hormone-regulated plant defences. Plant Pathology, 62, 43-55.

Vos, I.A., Verhage, A., Schuurink, R.C., Watt, L.G., Pieterse, C.M.J. and Van Wees, S.C.M. (2013b) Onset of herbivore-induced resistance in systemic tissue primed for jasmonate-dependent defenses is activated by abscisic acid. Frontiers in Plant Science, 4, 539.

Wang, W. and Wang, Z.-Y. (2014) At the intersection of plant growth and immunity. Cell Host \& Microbe, 15, 400-402. 
Wasternack, C. (2015) How jasmonates earned their laurels: past and present. Journal of Plant Growth Regulation, 34, 761-794.

Windram, O., Madhou, P., McHattie, S., Hill, C., Hickman, R., Cooke, E., Jenkins, D.J., Penfold, C.A., Baxter, L., Breeze, E., Kiddle, S.J., Rhodes, J., Atwell, S., Kliebenstein, D.J., Kim, Y.S., Stegle, O., Borgwardt, K., Zhang, C.J., Tabrett, A., Legaie, R., Moore, J., Finkenstadt, B., Wild, D.L., Mead, A., Rand, D., Beynon, J., Ott, S., Buchanan-Wollaston, V. and Denby, K.J. (2012) Arabidopsis defense against Botrytis cinerea: Chronology and regulation deciphered by high-resolution temporal transcriptomic analysis. the Plant Cell, 24, 3530-3557.

Yamaguchi-Shinozaki, K. and Shinozaki, K. (2006) Transcriptional regulatory networks in cellular responses and tolerance to dehydration and cold stresses. Annual Review of Plant Biology, 57, 781-803.

Yasuda, M., Ishikawa, A., Jikumaru, Y., Seki, M., Umezawa, T., Asami, T., Maruyama-Nakashita, A., Kudo, T., Shinozaki, K., Yoshida, S. and Nakashita, H. (2008) Antagonistic interaction between systemic acquired resistance and the abscisic acid-mediated abiotic stress response in Arabidopsis. Plant Cell, 20, 1678-1692. 


\section{Supporting Information}

Additional supporting information may be download via link

http://onlinelibrary.wiley.com/doi/10.1111/tpj.13167/full

\section{Supplementary Tables}

Supporting Table S1: Differentially expressed genes (DEGs) of Arabidopsis thaliana (AGI numbers of DEGs; FDR <0.05; >2-fold) in response to $B$. cinerea infection, $P$. rapae infestation, drought stress, and their six sequential combinations at four consecutive time points.

Supporting Table S2: Enriched GO terms in gene clusters shown in Figure 3 (Clustering of all DEGs of the single stresses).

Supporting Table S3: Enriched GO terms in gene clusters shown in Figure $4 \mathrm{~b}$ (Shared core DEGs between the three single stress responses).

Supporting Table S4: Enriched GO terms in gene clusters shown in Figure 5 (Dynamics of the expression of the $B$. cinerea set of DEGs during single and sequential double stresses).

Supporting Table S5: Enriched GO terms in Wigwams modules of $B$. cinerea, rapae, and Drought set of DEGs.

Supporting Table S6: Enriched GO terms in gene clusters shown in Figure 9 (Dynamics of the expression of the $P$. rapae set of DEGs during single and sequential double stresses).

Supporting Table S7: Enriched GO terms in gene clusters shown in Figure 12 (Dynamics of the expression of the drought set of DEGs during single and sequential double stresses).

Supporting Figure S1. Expression patterns of all 35 Wigwams modules from the $B$. cinerea set of DEGs during single and sequential stress conditions.

Supporting Figure S2. Expression patterns of all 93 Wigwams modules from the $P$. rapae set of DEGs during single and sequential stress conditions.

Supporting Figure S3. Expression patterns of all 72 Wigwams modules from the drought set of DEGs during single and sequential stress conditions. 


\section{$\mathbb{C} \| \Delta \mathbb{P} \mathbb{\|} \mathbb{E} \mathbb{R}$ (1)}

\section{Opposite interaction effects on the ethylene signalling pathway of two biotic stresses (Botrytis cinerea and Pieris rapae) on subsequent drought stress responses in Arabidopsis thaliana}

Pingping Huang ${ }^{1}$, Edouard Severing ${ }^{2}$, Nelson H. Davila Olivas ${ }^{3}$, Silvia Coolen ${ }^{4}$, Marcel C. van Verk ${ }^{4,5}$, Richard Hickman ${ }^{4}$, Alexander H. J. Wittenberg ${ }^{6}$, Martin de Vos $^{6}$, Marcel Prins ${ }^{6}$, Saskia C.M. van Wees ${ }^{3}$, Corné M.J. Pieterse ${ }^{3}$, Joop J.A. van Loon $^{2}$, Marcel Dicke ${ }^{2}$ and Mark G. M. Aarts ${ }^{1}$

${ }^{1}$ Laboratory of Genetics, Wageningen University, Droevendaalsesteeg 1, 6708 PB, Wageningen, the Netherlands.

${ }^{2}$ Laboratory of Entomology, Wageningen University, Droevendaalsesteeg 1, 6708 PB, Wageningen, the Netherlands.

${ }^{3}$ Plant-Microbe Interactions, Department of Biology, Utrecht University, P.O. Box 800.56, 3508 TB, Utrecht, the Netherlands

${ }^{4}$ Bioinformatics, Department of Biology, Utrecht University, P.O. Box 800.56, 3508 TB, Utrecht, the Netherlands

${ }^{5}$ Keygene N.V., P.O. Box 216, 6700 AE Wageningen, the Netherlands 


\section{Abstract}

It is important to understand how plant responses to the combination of biotic and abiotic stresses because plants are exposed to multiple biotic and abiotic stresses that occur either simultaneously or sequentially in the natural environment. The molecular mechanisms underlying plant responses to individual stresses have been studied extensively but less is known about the responses to a combination of stresses. In this study, we performed RNA-seq to discover the transcriptome changes of Arabidopsis thaliana to drought and rehydration, in combination with two biotic pre-treatments (necrotrophic fungal infection by Botrytis cinerea and herbivory by Pieris rapae larvae), in order to gain a deep understanding of the interaction between these combinations of stresses. We found drought stress has the strong influence of secondary messengers and photosynthesis and the one-day sequential rehydration period is insufficient to fully overcome the effects of drought stress on Arabidopsis. Herbivory by Pieris has a much higher impact on the transcriptome during sequential drought and rehydration, than the infection with Botrytis. The biotic pre-treatment can have strong antagonistic effects on sequential drought responses. Opposite effects were observed on 42 differential expressed genes (DEGs), including ethylene-related genes ERF5, ERF6, ERF104 and drought responsive gene P5CS1, during the subsequent drought treatment, revealing specific temporal regulation during the sequential drought that is influenced by the biotic pre-treatment factor. Genes with alternative splicing (AS) variants were identified in different stress conditions, but none of them were found among the DEGs, showing a separate regulation of gene expression and AS in response to stresses. Phenotypic differences found for the mdar 3 and pin 7 mutants suggest the MDAR3 and PIN7 genes to play an important role in the response to the combinatorial stress.

\section{Keywords}

Drought, Pieris rapae, Botrytis cinerea, (a)biotic stress response, Arabidopsis thaliana, RNA-sequencing, alternative RNA splicing. 


\section{Introduction}

Plants growing under natural conditions are often simultaneously or sequentially exposed to various biotic and abiotic factors which may interfere with plant growth and development causing a substantial annual reduction in agricultural production yield (Hirt and Shinozaki, 2004). Strong effects of (a)biotic factors are considered to be imposing plant stress, hence these factors are often referred to as conferring (a)biotic stress, and the tolerance to resist their effect as stress tolerance.

Plants respond to adverse biotic and abiotic conditions primarily through plant hormone-mediated pathways. This includes signalling pathways involving abscisic acid (ABA), which plays an important role in abiotic stress responses to e.g. drought and salt (Shinozaki and Yamaguchi-Shinozaki, 2007); jasmonic acid (JA) and ethylene (ET), which are essential in reacting to necrotrophic fungi and chewing insects; and salicylic acid (SA), which activates plant defence responses upon infection with biotrophic fungi (Pieterse et al., 2009; Dodds and Rathjen, 2010). Other plant hormones such as brassinosteroids (BR)s, auxins (AUX), and cytokinins ( $\mathrm{CK}$ ) are also involved in regulating the responses to biotic and abiotic stresses, but play a minor role (Hirt, 2009; Pareek et al., 2010; Pieterse et al., 2012).

The interaction between biotic and abiotic stress responses, mediated by different plant hormones, can be both synergistic or antagonistic. Negative interactions have been observed between JA and SA, ABA and SA, ABA and JA, and $A B A$ and $E T$; while positive interactions have been observed between $A B A$ and AUX (Yoshioka and Shinozaki, 2009). Studies in tomato indicate that drought stress leads to increased resistance to Botrytis cinerea infection (Audenaert et al., 2002; Achuo et al., 2006). Vice versa, interactions with pathogens and insects can also have a positive impact on plant responses to subsequent drought stress. For instance, pathogen-induced priming has a positive effect on plants in response to drought (Ramegowda and Senthil-Kumar, 2015), and prior infections of the Brome Mosaic Virus (BMV), Cucumber Mosaic Virus (CMV), or Tobacco Mosaic Virus can enhance Nicotiana benthamiana tolerance to drought (Xu et al., 2008). On the other hand, prior Turnip Mosaic 
Virus (TuMV) infection reduces plant biomass and leaf number under drought stress when compared to control conditions (Prasch and Sonnewald, 2015). Arabidopsis thaliana plants exposed to Pieris rapae larvae or to the necrotrophic fungus Botrytis cinerea, are expected to trigger JA-ET-mediated signalling through different branches within the JA-ET signalling pathway. The Botrytis response is regulated through the ETHYLENE RESPONSE FACTOR ('ERF') branch, which is co-regulated by ET, and the Pieris response is regulated through the 'MYC' branch, which is co-regulated by ABA (Pré et al., 2008; Vos et al., 2013). These two branches interact with each other antagonistically (Dombrecht et al., 2007). Genes from the two branches can also interact positively or negatively with drought-induced ABA signalling, eg. MYC2 (MYC branch) binds to the promoter of OCTADECANOID-RESPONSIVE ARABIDOPSIS AP2/ERF59 (ORA59) and $E R F 1$ (ERF branch) and negatively regulates their expression. Both targets are positive regulators of the Botrytis defence gene PDF1.2 (Anderson et al., 2004; Yoshioka and Shinozaki, 2009).

The ways by which plants respond to individual biotic and abiotic stress treatments have been studied extensively. Far less is known regarding plant responses to multiple stresses, either simultaneously or consecutively encountered. Recent studies have identified genes that play a role in response to multiple stresses by comparing transcriptome data from varying biotic and abiotic stresses (Narsai et al., 2013; Shaik and Ramakrishna, 2013; Sewelam et al., 2014; Ramegowda and Senthil-Kumar, 2015). The consecutive combination of exposure to biotic and abiotic stress evidently triggers specific multiple stressresponsive genes, indicating a tailored combinatorial stress signalling network (Atkinson and Urwin, 2012; Sewelam et al., 2014). However, the molecular responses in natural situations, where plants encounter simultaneous or consecutive combinations of biotic and abiotic stress, remain to be elucidated. To gain novel insights into the interaction between biotic and abiotic stress responses we studied the Arabidopsis transcriptome, including alternative RNA splicing, in response to drought as either a single drought-stress-inducing condition or following exposure to two different biotic stresses applied prior to the drought treatment. 
The purpose of this study was to: 1 ) observe the effects of the two prior biotic stresses on the subsequent drought treatment response, and 2) discover the differences of the effects of the two prior biotic stresses on subsequent drought. We identified a large number of differentially expressed genes (DEGs) as well as alternative splicing (AS) events under the two combinatorial treatments with both antagonistic or synergistic effects on plant performance. 


\section{Material and Methods}

\section{Plant material and growth}

Arabidopsis thaliana accession Col-0 seeds were vernalized at $4{ }^{\circ} \mathrm{C}$ in the dark for $48 \mathrm{~h}$ to break seed dormancy. Thereafter, seeds were sown on river sand with half-strength Hoagland nutrient solution containing sequestreen, as described Van Wees et al (2013). Plants were grown in a climate-controlled growth chamber ( $8 \mathrm{~h}$ day $/ 16 \mathrm{~h}$ night) set at $21^{\circ} \mathrm{C}$ and $70 \%$ relative humidity. Irradiation was set at $100 \mu \mathrm{mol} \mathrm{m} \mathrm{m}^{-2} \mathrm{~s}^{-1}$. Fourteen-day-old seedlings were transferred to individual pots containing a mixture of river sand and peat (1:1, $v: v)$. Water was supplied to the bottom of each pot three times per week.

\section{Experimental design}

The experiment was carried out in a fully randomized design with two factors: time and treatment. Four time points were selected for measurements, with $\mathrm{T} 1, \mathrm{~T} 2$, and $\mathrm{T} 3$ after 4, 5, and 6 days of drought treatment, respectively, which started after growing plants for 28 days (Figure 1 ). Directly after the last time point, plants were re-watered to rehydrate. T4 was set at 24 hrs (1 day) of rehydration. The experiment comprised four experimental groups: control, single drought, Botrytis infection followed by drought and Pieris exposure followed by drought. Randomized Block Design was used for this experiment. The climate chamber space was divided into three subgroups called blocks. Plants within each block are randomly subjected to different treatments.

\section{Preparation of Pieris rapae and Botrytis cinerea for treatments}

Pieris rapae caterpillars were reared on cabbage plants (Brassica oleracea convar. capitata var. alba) under greenhouse conditions $\left(24^{\circ} \mathrm{C}\right.$, with natural daylight). Butterflies were supplied with flowering plants such as Lantana camara for their food (nectar). When flowers were scarce, additional food (solution of $20 \%$ honey and $10 \%$ sucrose) was offered to the butterflies. Inbreeding of the population was minimalized by adding wild butterflies and caterpillars from the Dutch Flevopolder to the existing population. After starving for $1 \mathrm{~h}$, first-instar (L1) larvae were placed on Arabidopsis leaves using a fine paint brush as described (Van Wees et al., 2013). 
Botrytis cinerea strain B05.10 (Staats and Van Kan, 2012) was grown on half-strength Potato Dextrose Agar (PDA; Difco Laboratories) plates containing penicillin $(100 \mu \mathrm{g} / \mathrm{ml})$ and streptomycin $(200 \mu \mathrm{g} / \mathrm{ml})$ for 2 weeks at room temperature. Spores were collected, filtered through glass wool, and resuspended in half-strength Potato Dextrose Broth (PDB; Difco Laboratories) to a final density of $1 \times 10^{5}$ spores/ml. After a $3 \mathrm{~h}$ incubation period, the spores were used for inoculation by applying two $5-\mu$ droplets on one Arabidopsis leaf as described (Van Wees et al., 2013).

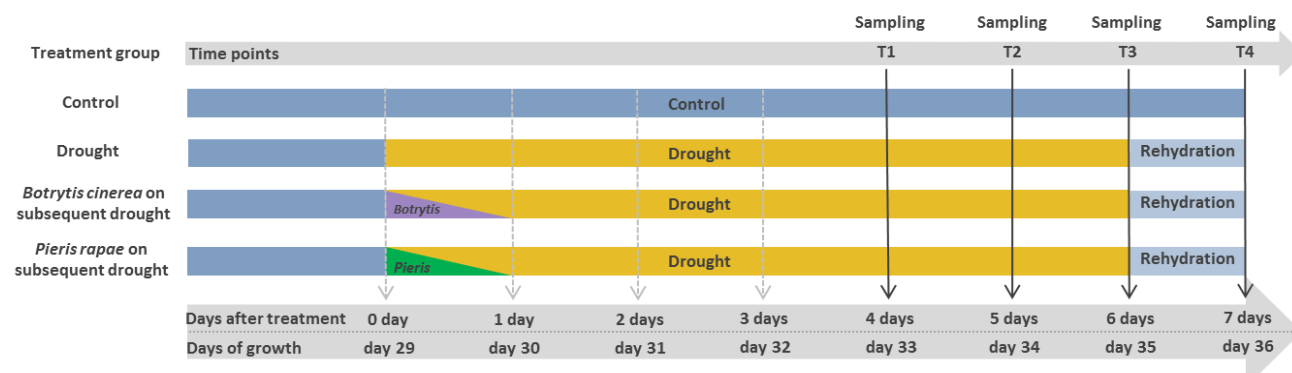

Figure 1. Experimental design for treatment of Arabidopsis plants subjected to drought, Botrytis cinerea infection and drought, or Pieris rapae herbivory and drought, and control conditions. Conditions are indicated in different colours: control (dark blue); drought (yellow); Botrytis pre-treatment (purple); Pieris pre-treatment (green) and rehydration after drought (light blue). Vertical arrows indicate sampling time points (T1-T4).

\section{Drought and combinatorial stress treatments}

For the single drought treatment, 28-day-old plants of Arabidopsis accessions were withheld from water for 6 days. Afterwards, plants were rewatered and allowed to recover for 1 day. For the combination of Botrytis exposure and subsequent drought, a Botrytis pre-treatment was performed right after the last watering before the drought treatment, by inoculating leaves 6 and 7 of each plant with two $5-\mu$ droplets of a Botrytis spore suspension $\left(1 \times 10^{5}\right.$ spores $/ \mathrm{ml}$ ) per leaf and placing the plants at $100 \%$ relative humidity $(\mathrm{RH})$ for 1 day (Figure 1). The next day, the $\mathrm{RH}$ was reduced to that of drought-exposed plants. For the combination treatment of Pieris and subsequent drought, the Pieris pre-treatment was performed at the same time as the Botrytis pretreatment by placing one Pieris caterpillar on leaf 7 and allowing it to feed on the plant for 1 day. Plants without receiving any treatment, neither biotic nor abiotic, were used as control. Leaf number 8 was harvested, if undamaged, after 
4, 5 and 6 days of withholding water (T1, T2, T3, respectively) and at 1 day after the rehydration treatment (T4)(Figure 1 ).

The treatments for the T-DNA insertion mutants were the same as described for the accessions. For information on the identity of T-DNA insertion mutants, see supplementary table 1 .

\section{RNA extraction and Iibrary preparation}

RNA was extracted using the Plant RNeasy Plant Mini Kit (Qiagen, cat. no. 74903), according to the manufacturer's instructions. All samples were treated with DNase I on a column using the Qiagen RNase-Free DNase Set (cat. no. 79254). Quality of RNA was evaluated by determining the RNA Integrity Number (RIN) with an Agilent 2100 bioanalyzer and RNA LabChip. For the library preparation, samples with a RIN value $\geq 6$ were used. The samples were processed according to the TruSeq Stranded mRNA HT Sample Prep Kit from Illumina. This protocol allows the identification of strand-specific transcripts. First, poly-A RNA was isolated from total RNA using poly-T oligo-attached magnetic beads. Subsequently, poly-A RNA was fragmented using divalent cations under elevated temperature. First-strand cDNA was synthesized using random primers. Strand specificity was achieved by replacing dTTP with dUTP in the second Strand Marking Mix (SMM), followed by second strand CDNA synthesis using DNA polymerase I and RNase $\mathrm{H}$.

\section{RNAseq analysis, Differential expressed genes, Alternative RNA splicing}

Samples were sequenced on an Illumina Hi-Seq 2000 sequencing machine randomly assigned to seven lanes of three Illumina flow cells. Sequence reads were quality trimmed and mapped to the Arabidopsis thaliana genome version 'TAIR 10' (www.arabidopsis.org) using TopHat with parameters: -N 3 --minintron-length 50 --max-intron-length 5000 -g 1 -M -p 4 -G --read-edit-dist 3 -library-type (Trapnell et al., 2009). The number of reads mapped to each annotated Arabidopsis gene (TAIR10) was determined using HTSeq count 
(Anders et al., 2015). Read counts were subjected to a regularized log transformation, implemented in DESeq2 (Love et al., 2014).

Differentially expressed genes (DEGs) were computed per time point using the DESeq2 Bioconductor package in R (Anders and Huber, 2010; Love et al., 2014). To determine DEGs under drought treatment, we compared drought with control. To determine DEGs under the combinational stresses, we compared each combinatorial treatment to the single drought treatment. To determine DEGs under rehydration treatment, we compared the rehydration to the control.

To determine alternative RNA splicing, putative full-length transcripts were assembled for each RNAseq sample using cufflinks package (Trapnell et al., 2012). The full transcriptome was obtained by merging the individual assemblies using cuffmerge from the cufflinks package. Custom python scripts were used for detecting the following splicing events: intron retention, alternative donor site, alternative acceptor sites, exon skipping and mutually exclusive exons (Davila Olivas et al., 2016).

Mixture of Isoforms Probalistic model (MISO) was used for quantifying alternative splicing events in each individual sample and the pooled replicates for each condition (Katz et al., 2010). For each sample (excluding pooled datasets), those alternative splicing events that were not supported by at least 20 isoform-specific reads were discarded. Differential splicing events were detected in 2 steps:

1. The alternative splicing (AS) events for each pairwise condition were analysed as follows: All events for which either of the two conditions lacked more than one replicate value were discarded. It was required that the values of the replicates in each condition were closer to each other than to any other replicate value in the other condition.

2. For each splicing event that passed the filtering, we tested whether the corresponding values in the pooled set were significantly different from each other. The significance in MISO is provided as Bayes factors, which describe how much more likely the event is differentially regulated than 
not. Splicing events with a difference of at least 0.05 and a Bayes factor of $\geq 4$ were considered as significant events.

\section{Enriched gene ontology, metabolic pathway, transcription factors analysis}

Enriched gene ontology (GO), metabolic pathways, and transcription factors (TFs) were identified using the online tool PlantGSEA (http://structuralbiology.cau.edu.cn/PlantGSEA/) (Yi et al., 2013). In all cases, we used the following parameters: hypergeometric test with BenjaminiHochberg FDR adjustment, $a=0.05$. As background, we used only genes that were expressed in at least one sample out of all samples that were analysed.

\section{Statistical analysis}

One-way ANOVA was used to compare the areas of each leaf consumed by Pieris in the Pieris pre-treatment. Two-way ANOVA was used to compare plants in response to drought (compared to control) and to compare plants in response to the combination of Pieris on subsequent drought (compared to drought). In all cases $p$-values $<0.05$ were considered as significant.

\section{Real-time quantitative reverse transcriptase (qRT)-PCR}

$1 \mu \mathrm{g}$ total RNA was used for reverse transcription by iScript CDNA synthesis kit (Bio-Rad); $25^{\circ} \mathrm{C}$ for $5 \mathrm{~min}, 42^{\circ} \mathrm{C}$ for $30 \mathrm{~min}$ and $85^{\circ} \mathrm{C}$ for 5 min. CDNA was diluted ten times for Quantitative Reverse Transcriptase-PCR (qRT-PCR) use. The At3g15930 (PCMP-E51) gene was used as the reference gene upon selection through the "References" web tool (http://refgenes.org/rg) (forward primer sequence 5'-ACCAAACCCAACATTCCAAA-3' and the reverse primer sequence $3^{\prime}$ ACACAACCACGTCTGGTTCA-5'). The qRT-PCRs were performed using iQ SYBR ${ }^{\circledR}$ Green Supermix (Bio-Rad, cat. no. 170-8885) on a Bio-Rad CFX96 real-time PCR system, set at $95{ }^{\circ} \mathrm{C}$ for 4 minutes, followed by 40 cycles of $95{ }^{\circ} \mathrm{C}$ for 10 seconds and $55{ }^{\circ} \mathrm{C}$ for 30 seconds. The primers of the PIN-FORMED 7 (PIN7) gene and the two AS variants are shown in Supplementary Table 12.

\section{Results}




\section{Differentially expressed genes by drought and subsequent}

\section{rehydration}

A time-course experiment was performed using Arabidopsis $A$. thaliana plants which were separately exposed to two biotic stresses: fungal infection by Botrytis cinerea and herbivory by Pieris rapae caterpillars, followed by drought ("Botrytis and subsequent drought" and "Pieris on subsequent drought") (Figure 1). In addition to the combinatorial treatment, plants were subjected to a single drought treatment without a pre-treatment ("drought"). Samples were harvested after 4 (T1), 5 (T2) and 6 days of drought (T3) and one day later, meaning after 24 hrs of rehydration(T4) (Figure 1). Gene expression was determined using RNA-seq.

a.

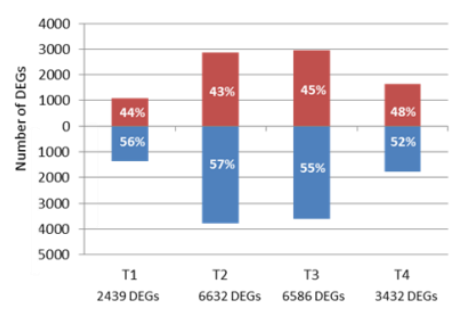

b.

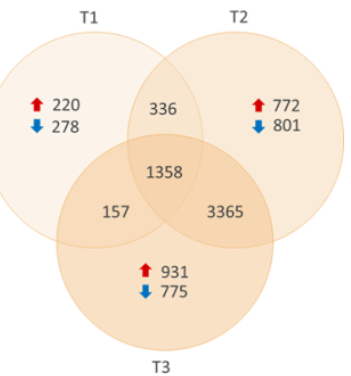

c.

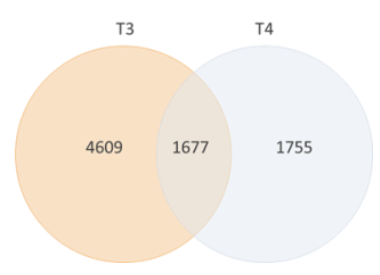

Figure 2. Bar plot (a.) showing the number of up- and down-regulated differentially expressed genes (DEG) at four time points, after (T1), 5 (T2) and 6 days of drought (T3), and after one-day sequential rehydration (T4). The $Y$ axis represents the number of DEGs and the $X$ axis represents time points and the total number of DEGs. The ratio of up- (red) or down-regulated (blue) genes are indicated. Venn diagram showing the numbers DEGs of Arabidopsis Col-0 (b.) at three time points, after 4 (T1), 5 (T2) and 6 days of drought (T3), when compared to well-watered control plants at each time point, the numbers of up- or down-regulated genes are indicated with blue and red arrows. Or (c.) at two time points, after 6 days of drought (T3) and after one day rehydration (T4), when compared to well-watered control plants at each time point.

A total of 8993 differentially expressed genes (DEGs) were identified when plants exposed to drought at time points T1, T2 and T3 were compared with plants grown on control conditions (Figure 2.a; Supplementary Table 2). These expression differences illustrate the effects of drought on gene expression. A total of 2349 DEGs were identified at T1, 6632 DEGs at T2 and 6586 DEGs at T3, which are all one day apart in the drought response, suggesting that the effect of the treatment became more severe the longer it lasted. In total, 1358 DEGs 
were shared by the three time points, which can be considered to be common drought-responsive genes (Figure 2.b; Supplementary Table 2). With so many DEGs, drought causes major changes to the transcriptome and is therefore be considered to be a severe stress.

To observe if rehydration upon drought reverts plants to the same state as seen under control conditions, plants exposed to rehydration after drought treatment were compared to the control (not drought-stressed), which still revealed 3432 DEGs (Supplementary Table 3). Comparing DEGs under rehydration (T4) to DEGs under drought at T3, we found that 1677 drought responsive genes were affected by the sequential rehydration treatment (Figure 2.c; Supplementary Table 3.e). This means that a one-day rehydration period is insufficient to fully overcome the effects of drought stress on Arabidopsis.

\section{Enriched GO terms of genes involved in the drought and subsequent rehydration response}

The 1358 common drought-responsive DEGs (Figure 2) were examined for overrepresented gene ontology (GO) terms. The top 20 enriched GO terms indicates three main gene expression responses to drought: the defence response, which includes GO terms such as response to stimulus, response to abiotic stimulus, response to chemical stimulus, response to stress, response to temperature stimulus; the photosynthesis response, including GO terms photosynthesis and light reaction; and the metabolic processes response, including GO terms such as secondary metabolic process, oxoacid metabolic process, carboxylic acid metabolic process, etc. (Supplementary Table 3.a). To determine the role of DEGs involved in a biochemical pathway, an 'enriched pathway analysis' was performed, which identified 32 pathways that were associated with the DEGs, including the biosynthesis of phenylpropanoids, biosynthesis of plant hormones, and photosynthesis (Supplementary Table 3.b). In addition, the targets of several transcription factors that are related to stress responses are enriched, such as transcription factors HY5 (light response), AtbHLH15 (light and hormone response), AP2, MYB88, and MYB124 (abiotic stress response), WRKY53 (drought response), and MYC2 (drought and biotic 
stress response) (Osterlund et al., 2000);(Xie et al., 2010; Mizoi et al., 2012) (Supplementary table 3.c).

When analysing the large set of DEGs identified when comparing the gene expression after rehydration with gene expression in unstressed, control, plants (Supplementary Table 4.a) the GO terms that are associated with defence response are still enriched, as they were upon drought treatment (Supplementary Table 4.b). DEGs involved in the biosynthesis of alkaloidderived pathways and biosynthesis of phenylpropanoids were also overrepresented (Supplementary Table 4.c), as were targets of transcription factors AtbHLH15 (involved in light and hormone response), AP2, MYB88, and MYB124 (involved in abiotic stress response), WRKY53 (involved in drought response) AGL15 (involved in flowering), and PYE (involved in the response to iron deficiency) (Adamczyk et al., 2007; Long et al., 2010) (Supplementary Table 4.d) and several DEGs known to be drought-responsive, such as RESPONSIVE TO DESICCATION 29a (RD29a), RESPONSIVE TO DESICCATION 29a (RD29b), and DEHYDRATION-RESPONSIVE ELEMENT-BINDING PROTEIN 2a (DREB2a) (Shinozaki and Yamaguchi-Shinozaki, 2007). In conclusion, one day of rehydration did not dramatically revert the drought-induced gene expression profile back to normal.

Comparing DEGs under rehydration to DEGs under drought at T3, a set of 1677 DEGs were shared by the two time points. Enriched GO terms that are associated with stress responses such as response to stimulus, response to chemical stimulus, response to stress, response to endogenous stimulus, and response to abiotic stimulus are highly enriched (Supplementary Table 4.e). DEGs involved in the metabolic pathways, biosynthesis of plant hormones, and biosynthesis of alkaloids are overrepresented (Supplementary Table 4.f). We did not find overrepresented transcription factor targets in this gene set (Supplementary Table 4.g). 


\section{Differential expressed genes by combinatorial and sequential rehydration}

Plants exposed to the two combinatorial stresses were compared with plants subjected to the single drought treatment, at comparable time points, to determine if there is an effect of a biotic pre-treatment on the response to drought (Figure 1). For the combination of Botrytis followed by drought, 101 DEGs were identified (Figure 3.a), most of them representing down-regulated genes, and the large majority of them (80) were differentially expressed at T2 (Supplementary Table 5.a). Only one DEG appeared in two time points (T2 and T3), the AMINO ACID PERMERASE 6 (AAP6) gene (At5g49630), encoding a highaffinity Asp/Trp uptake transporter to transport amino acids in phloem sap (Hunt et al., 2010).

A total of 734 DEGs were identified when comparing the combined Pieris and subsequent drought treatment to the drought treatment (Figure 3.b, Supplementary Table 6). The number of DEGs decreased over time from 503 (T1) to 20 (T3). Most DEGs were down-regulated at T1, while most DEGs were upregulated at T2 and T3. In total 165 DEGs were common between T1 and T2, but only 10 DEGs between T2 and T3, and 7 between T3 and T1. Of the 20 DEGs found at T3, no less than six were common to all three time-points. Genes including two known drought-responsive genes CALCIUM-DEPENDENT PROTEIN KINASE 28 (CPK28) (At5g66210) and EARLY RESPONSE TO DEHYDRATION 6 (ERD6) (At1g08930) were expressed at all three time points (Kiyosue et al., 1998; Zou et al., 2015). When comparing the combinatorial treatments, 49 of all 786 DEGs were found in both treatments (Figure 4.a).

To investigate if the biotic pre-treatments still had any effect on gene expression after rehydration to recover from the drought treatment, DEGs were identified when comparing the gene expression upon the three rehydration treatments (after drought alone and after Botrytis/Pieris pre-treatment and drought). The Botrytis pre-treatment affected the expression of only 24 genes (Supplementary Table 7), while the Pieris pre-treatment affected the expression of 1779 genes (Supplementary Table 8). Only 7 DEGs, including gene CHALCONE SYNTHASE (CHS) (At5g13930) which is required for purple 
anthocyanins accumulation in leaves and stems (Li and Strid, 2005), were found to be common for both combinatorial treatments upon rehydration (Figure 4.b, Supplementary Table 9.b). This illustrates that herbivory by Pieris rapae has a much higher impact on the transcriptome during sequential drought and rehydration, than the infection with Botrytis cinerea.

a. Botrytis and subsequent drought

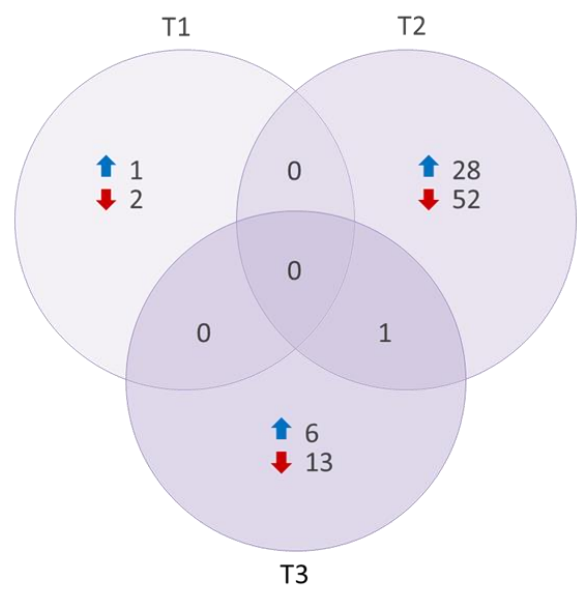

b. Pieris and subsequent drought

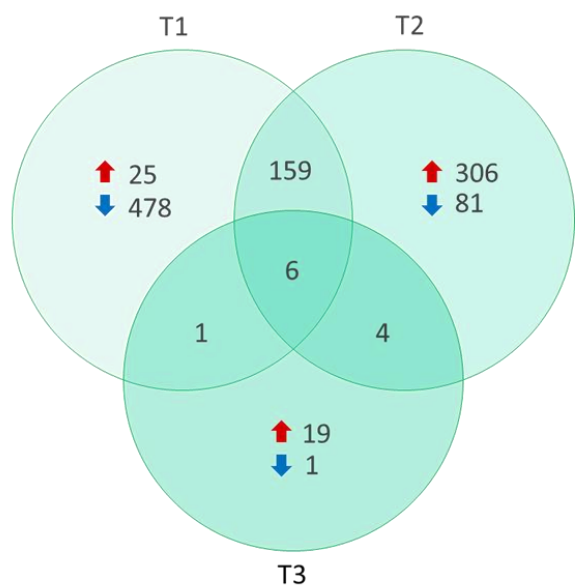

Figure 3. Venn diagram showing the numbers of DEGs of Col-0 at points T1-T3 (see figure 1) when comparing plants treated with (a) Botrytis cinerea and drought (purple;) or (b) Pieris rapae and drought (green) with the drought alone treatment. The numbers of up- or down-regulated genes are indicated with blue and red arrows.

a. DEGs in combinatorial conditions

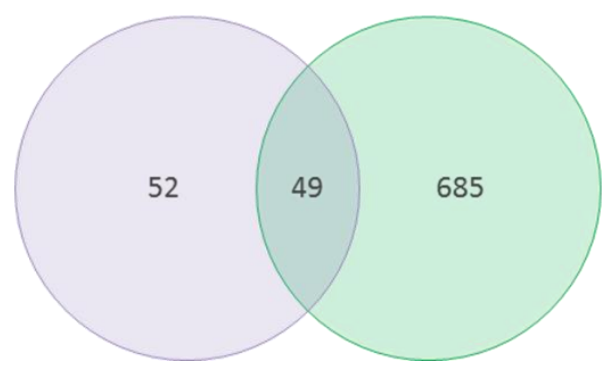

b. DEGs in sequential rehydration condition

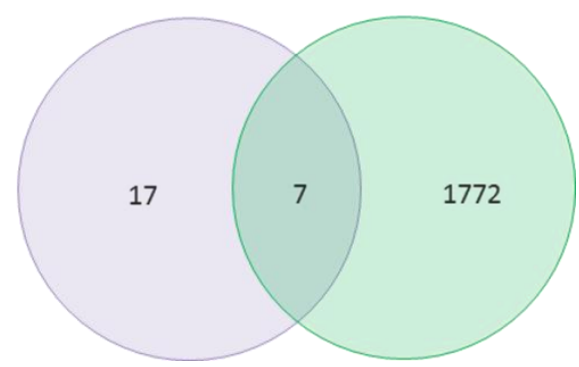

Figure 4. Venn diagram showing the numbers of DEGs of Col-0 when (a) comparing plants pre-treated with (Botrytis cinerea (purple) or Pieris rapae (green) followed by drought to control plants only treated with drought; or (b) when comparing plants pretreated with Botrytis cinerea (purple) or Pieris rapae (green) followed by drought and subsequent rehydration, to control plants treated only with drought and rehydration. The numbers of up- or down-regulated genes are indicated with blue and red arrows . 


\section{Enriched GO terms in combinatorial stress response}

Among the 101 DEGs identified under the stress combination of Botrytis on subsequent drought treatment. Genes including drought responsive gene $R D 28$ (At2g37180) (Taji et al., 1999), cytokinin-related gene CYTOKININ OXIDASE 5 (CKX5) (At1g75450) (Werner et al., 2003), auxin-related gene ARABIDOPSIS THALIANA METHYL ESTERASE 18 (ATMES18) (At5g58310) (Yang et al., 2008) (Supplementary Table 5.b). Among the 734 DEGs responsive to the Pieris pretreatment before subsequent drought, defence response associated GO terms such as response to chitin, defence response, innate immune response, response to stress, response to biotic stimulus, and regulation of defence responses were highly enriched (Supplementary Table 6.f). Known stressresponse genes include mitogen-active protein kinase (MAPK)-related genes such as MPK3 (At3g45640), MKK4 (At1g51660), MKK5 (At3g21220), and MAP kinase substrate 1 (MKS1, At3g18690). The DEGs involved in metabolic pathways such as plant-pathogen interaction pathway was especially enriched in this gene set (Supplementary Table 6.g), as well as targets of transcription factors such as ATBZIP60 (unfolded protein response) and GL1 (development, anthocyanin biosynthesis) were only enriched in this gene set (Humbert et al., 2012)(Supplementary Table 6.h).

\section{Common genes affected by both biotic pre-treatments in combination with drought}

A total of 49 DEGs were shared by both combinatorial treatments (Supplementary Table 9). Among the 49 DEGs, 17 DEGs were down-regulated and 14 DEGs were up-regulated under these treatments. Remarkably, for 42 genes the biotic pre-treatments had an opposite effect on the up- or downregulation of their expression, either at one or more time points. Among these are known stress response genes, such as ETHYLENE RESPONSIVE ELEMENT BINDING FACTOR 5 (ERF5) (At5g47230), ERF6 (At4g17490), ERF104 (At5g61600) and Delta1-Pyrroline-5-Carboxylate Synthase 1 (P5CS1) (AT2G39800). In general, these genes showed an initial down-regulation and subsequent up-regulation upon pre-treatment with Botrytis, and an initial upregulation and subsequent down-regulation upon the Pieris pre-treatment. This 
reveals specific temporal regulation during drought that is influenced by the biotic pre-treatment factor.

\section{CCR2 and MDAR3 play a role in response to the consecutive combination of Pieris and subsequent drought}

Genes that showed a high fold-change in expression when comparing the Pieris plus drought treatment with the drought treatment alone, were selected for further analysis of their relevance for the response. Phenotypic analysis was performed on T-DNA insertion mutants of three up-regulated candidate genes (WALL ASSOCIATED KINASE (WAK)-LIKE 10; WAKL10; At1g79680) (expression fold change (FC)>7), CINNAMOYL COA REDUCTASE; CCR2; At1g80820) (FC>12), MONODEHYDROASCORBATE REDUCTASE 3; MDAR3; At3g09940) (FC>30), and one down-regulated gene, At3g10720, encoding an invertase/pectin methylesterase inhibitor (FC<0.2) (Supplementary Table 6). The wakl10 mutant plants exhibited reduced rosette dry weight when compared with their Col-0 wild-type under control conditions (Figure 5.a). No mutants exhibited significant differences in response to Pieris treatment when compared to the wild type for leaf eaten area (Figure 5.b). Under drought stress, mdar3 mutants showed increased rosette dry weight when compared with the wild type (Figure 5.c). A decreased rosette dry weight was observed for the ccr2 and mdar3 mutants when pre-treated with Pieris followed by drought, when compared to the wild type (Figure 5.d; Supplementary Figure 2). This means that only for the mutant of At3g10720 we did not observe any effect on the phenotype under any of the tested conditions. All other genes are somehow involved in the growth of plants, with particularly CCR2, encoding a cinnamoyl CoA reductase involved in lignin biosynthesis, and MDAR3, encoding a monodehydroascorbate reductase, to be important to maintain wild-type growth under the combined Pieris-drought combination. 
a.

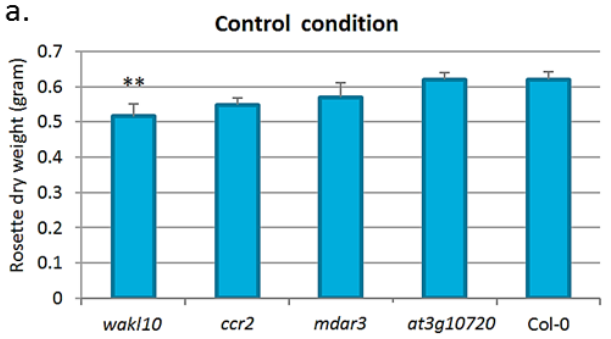

c.

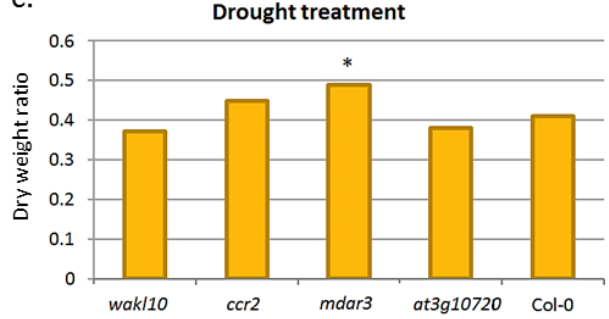

b.

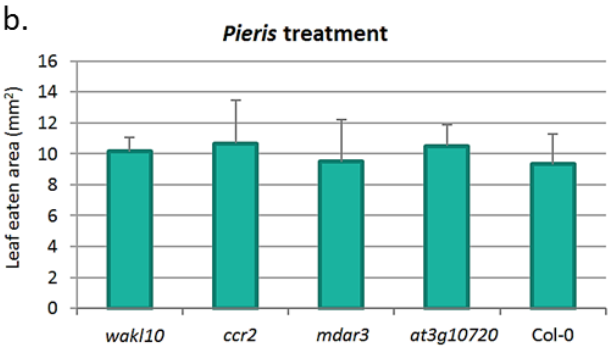

d.

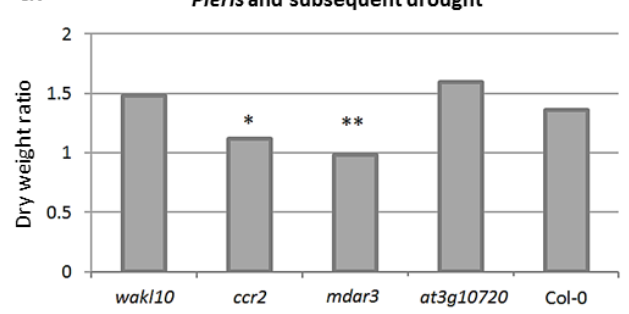

Figure 5. Phenotypes of T-DNA insertion mutants of DEGs WAKL10 (At1g79680), CCR2 (At1g80820), MDAR3 (At3g09940) and unnamed gene At3g10720, identified when comparing Pieris rapae pre-treated with non-pre-treated plants subjected to drought (Sup. Table .....).

a. Rosette dry weight of Col-0 wild type and mutant plants grown in control conditions.

b. Leaf areas eaten by Pieris of these genotypes.

c. Rosette dry weight ratios of these genotypes after exposure to drought, compared to control plants.

d. Rosette dry weight ratios of these genotypes after pre-treatment with Pieris and subsequent exposure to drought, compared to plants exposed to drought only.

One-way ANOVA was used for Figures 5.a and b. Two-way ANOVA was used for Figures 5.c and d. Error bars were calculated from at least six plants. * indicates mutants with a significant difference when compared to Col-0 wild type $(* p<0.05, * * p<0.01)$. 


\section{Alternative RNA splicing affecting gene expression upon exposure to stress-inducing conditions}

Alternative splicing (AS) is known to be sometimes an important factor in controlling the gene expression response to environmental perturbations (Staiger and Brown, 2013). To determine if there is any differential alternative RNA splicing, plants exposed to drought were compared to control plants at each of the three time points. For 19 genes a total of 23 AS variants were found (Table 1; Supplementary Table 10.a). When including the rehydration treatment in the analysis, and comparing rehydrated plants after drought exposure to plants continuously grown under control conditions, 17 genes with a total of 20 AS variants were found (Table 1; Supplementary Table 10.b).

When examining both plants exposed to biotic pre-treatments plus drought to plants only exposed to drought, five genes with as many AS variants were found for the Botrytis pre-treatment and 16 genes corresponding to 16 AS variants for the Pieris pre-treatment. There was no overlap between these two sets. When including the rehydration treatment, only the Botrytis pre-treatment was found to yield AS variants, for 5 genes (Table 1; Supplementary Table 10. c and d).

Table 1. Number of alternative splicing variants induced by different stresses

\begin{tabular}{|c|c|c|c|c|c|c|}
\hline Type & Dreatment & BD* & PD* & Rehydration after drought & Rehydration after BD & Rehydratoin after PD \\
\hline A3 & 10 & 1 & 5 & 4 & 4 & 0 \\
\hline A5 & 3 & 3 & 5 & 2 & 0 & 0 \\
\hline RI & 6 & 1 & 3 & 7 & 1 & 0 \\
\hline MXE & 1 & 0 & 2 & 1 & 0 & 0 \\
\hline SE & 3 & 0 & 1 & 16 & 5 & 0 \\
\hline Total & 23 & 5 & 16 & 14 & 1 \\
\hline
\end{tabular}

$\mathrm{A} 3$ = Alternative splicing at $3^{\prime}$ of intron; $\mathrm{A} 5=$ Alternative splicing at $5^{\prime}$ of intron, $\mathrm{RI}=$ Intron retention, $\mathrm{MXE}=$ mutually exclusive exon, $\mathrm{SE}=$ exon skipping. $\mathrm{BD}=$ combination of Botrytis and subsequent drought; PD = combination Pieris and subsequent drought. 


\section{PIN7 plays a role in response to Pieris, drought, and the consecutive stress combination treatments}

One gene, for which AS variants were found, that caught our attention is PIN-FORMED 7 (PIN7) (At1g23080), as it is known to be involved in auxin transport contributing to root architecture (Lewis et al., 2011). We found two splicing variants for this gene, PIN7-1 and PIN7-2, differing at the $5^{\prime}$ splice donor site of intron 1 leading to addition/removal of 4 amino acids (Figure 6.a). The splicing affects a region of the protein that is located in the cytoplasm. The expression of these two AS variants of PIN7 was determined using specific splice-site-flanking primers, which showed that the expression level of the PIN71 variant is significantly higher than of PIN7-2, under the single drought and the Pieris plus subsequent drought treatments (Figure 6.b). PIN7-1 was marginally, though significantly, higher expressed when plants were pre-treated with Pieris than when plants were only drought-treated. In order to verify if this gene has any phenotypic importance for the response to Pieris and/or drought, a T-DNA insertion pin7 mutant was obtained and grown under the different conditions. This mutant showed some remarkable phenotypic differences when compared to the wild type. When grown under control, non-stressing, conditions the pin7 mutant rosettes comprised significantly more dry biomass than Col-0 (Figure 7.a), of which much less was consumed by Pieris (Figure 7.b). However, when plants were exposed to drought, the pin 7 mutants performed much worse than Col-0 in terms of rosette dry biomass ratios when comparing drought-treated to well-watered plants (Figure 7.c). Pre-treatment with Pieris alleviated this strong inhibition by drought somewhat, though the pin 7 mutants stayed notably smaller than Col-0 plants (Figure 7.d). This suggests that PIN7 is important for Col-0 tolerance to drought although it enhances herbivory by Pieris. 
a.

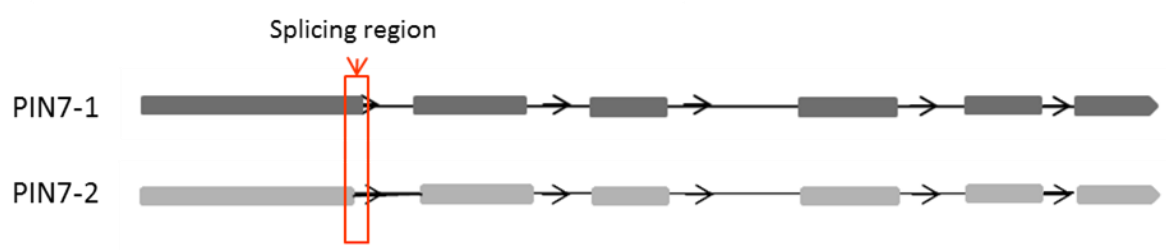

b.

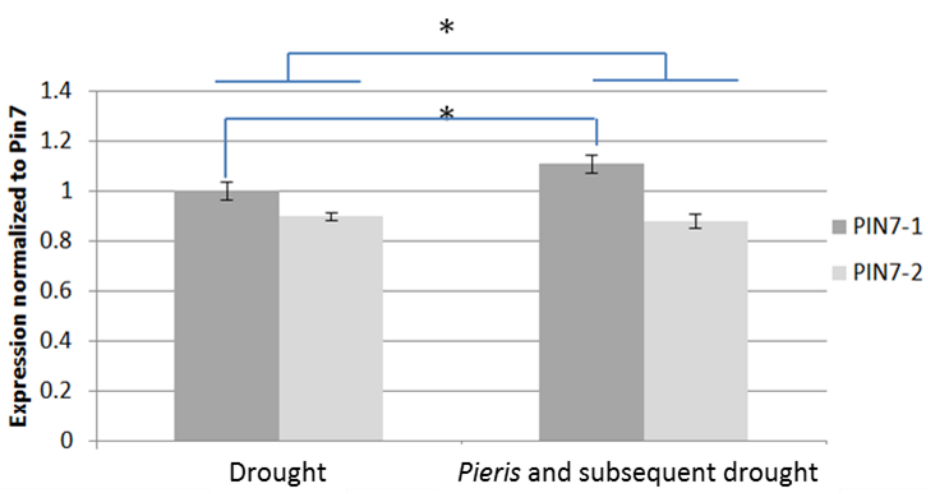

Figure 6 . Alternative splicing (AS) variants of PIN7 are differentially expressed upon Pieris and drought exposure.

a. Two splicing variants of PIN7, indicating the alternatively spliced region with a red box.

Exons are indicated as grey boxes and introns with lines, arrows indicate direction of transcription. AS variant PIN7-1 is indicated in dark grey and PIN7-2 in light grey.

b. Relative expression of the two PIN7 splicing variants when comparing drought treated with well-watered control plants, and comparing the Pieris and subsequent drought with the well-watered control plants.

A Student's t-test was used to compare expression of each splicing variant in the different conditions. Two-way ANOVA was used to test the differences of the two AS variants between different conditions. * indicate significant differences $(p<0.05)$. Standard error bars were calculated from at least six plants. 


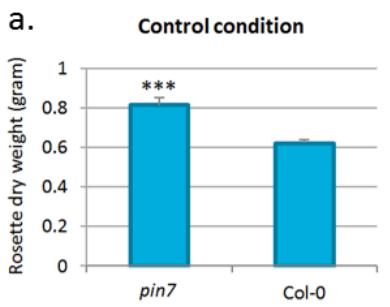

d. Pieris and subsequent drought

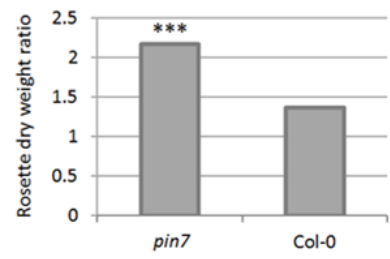

b. Pieris treatment

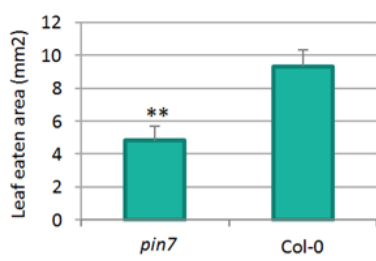

C.

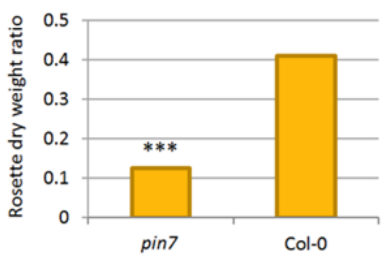

e.

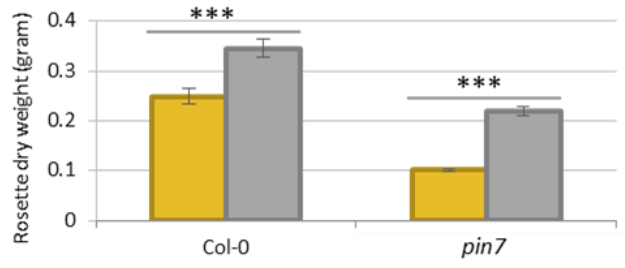

$\square$ Drought $\square$ Pieris and subsequent drought

Figure 7. The PIN7 gene is important for Arabidopsis tolerance to drought and herbivory.

a. Rosette dry weight of pin 7 mutant and Col-0 wild-type plants when grown in control conditions.

b. The area of rosette leaf consumed by Pieris of pin7 mutant and Col-0 wild-type plants.

c. Rosette dry weight ratios of pin 7 mutant and Col-0 wild-type plants when comparing drought-treated plants to well-watered plants.

d. Rosette dry weight ratios of pin 7 mutant and Col-0 wild-type plants when comparing Pieris pre-treated and drought exposed plants to plants only exposed to drought.

e. Rosette dry weight of plants in drought and the combinatorial conditions.

A student's t-test was used for a and b. Two-way ANOVA was used for $c$ and d. Error bars were calculated from at least six plants. *indicate significant differences, $* * p<0.01$, $* * * p<0.0001$. 


\section{Discussion}

\section{Transcriptomic responses to drought and subsequent rehydration}

In response to myriad biotic and abiotic stress triggers, plants mobilize signaling molecules thereby boosting the accumulation of so-called second messengers, such as reactive oxygen species (ROS), calcium ions $\left(\mathrm{Ca}^{2+}\right)$, phospholipids, mitogen-activated protein kinases (MAPKs), Sucrose NonFermenting-1 (SNF1)-related protein kinases (SnRKs), and phytohormones (Takahashi et al., 2011; Tuteja and Sarvajeet, 2012; Smékalová et al., 2014). Some genes related to secondary messengers and plant hormones were found among the 1358 common drought-responsive DEGs, such as the up-regulation of SnRKs family genes such as SnRK2.6 and SnRK2.7, plant hormones-related genes such as AUXIN RESPONSE FACTOR 2, AUXIN-INDUCED PROTEIN 13 (IAA13), JAMONATE-ZIM-DOMAIN PROTEIN 9 (JZA9), and ABSCISIC ACID RESPONSIVE ELEMENT-BINDIGN FACTOR 2/3 (ABF2/3); and down-regulation of CALCIUM-DEPENDENT PROTEIN KINASE 31 (CPK31), and plant hormone-related genes such as ET-stabilized transcription factors ETHYLENE INSENSITIVE 3-LIKE 1 (EIL1), auxin biosynthesis gene YUCCA9 (Supplementary Table 2). . Many LIGHT HARVESTING CHLOROPHYLL A/B BINDING (LHCB) protein genes were down-regulated under drought and the sequential rehydration. The $\angle H C B$ family genes encode proteins that are part of the light harvesting antenna that receives and transfers light energy to the reaction centre of photosystem II (PSII) (Jansson, 1994). The $\angle H C B$ genes are required for ABA-mediated stomatal aperture and the $I h c b$ knock out mutants were found incapable to control stomatal aperture under ABA treatment by (Xu et al., 2012). The downregulation of the $\angle H C B$ family genes under drought thus reflects the effect of drought-induced stomatal closure, inhibiting gas exchange. Thus, limiting $\mathrm{CO}_{2}$ assimilation and impairs photosynthesis.

A set of 1677 DEGs were expressed at T3 under drought as well as under the sequential rehydration treatment (T4). $30 \%$ of the DEGs were overlapped with the common drought-responsive DEGs. GO terms 'response to chemical stimulus' and the plant hormone biosynthesis pathway are overrepresented in 
this data set, both indicating hormone-related signalling to be involved. Plant hormone-related genes exhibited different expression at T4 in comparison to T3. Ethylene-related genes such as ERF6 and ERF8 were down-regulated in both conditions. JASMONATE-ZIM-DOMAIN-PROTEIN (JAZ) 3/7/8/9 and INDOLE-3ACETIC ACID INDUCIBLE (IAA) $13 / 18$ were up-regulated under in both conditions, while IAA29 and OCTAEDECANOID-RESPONSIVE ARABIDOPSIS AP2/ERF59 (ORA59) were down-regulated under drought, but up-regulated under the sequential rehydration condition. JAZ family genes function in signal transduction in JA signalling (Chini et al., 2007), and the same holds for the pathogen defence responsive gene ORA59, linking it to the ET signalling pathway (Pre et al., 2008). The auxin biosynthesis YUCCA 2/8/9 genes and the auxin efflux carrier PIN-FORMED 4 (PIN4) gene were also down-regulated under drought and up-regulated under sequential rehydration, indicating an inhibition of auxin biosynthesis and transport in plants by drought. ABA-related genes such as DREB2a, RESPONSIVE TO DEHYDRATION 22 (RD22), RESPONSIVE TO DESICCATION 29a (RD29a), RESPONSIVE TO DESICCATION 29b (RD29b) were up-regulated under drought at T3 (day7) but down-regulated in sequential rehydration condition. This is showing that the well-watered state is indeed sensed by plants and that for mostly down-stream drought responsive genes are still expressed upon rehydration.

\section{Temporal regulation of genes influenced by biotic pre-treatment}

A set of 49 identified DEGs were shared by the two combinatorial treatments, of which 31 genes exhibited opposite directions of expression response when comparing the two pre-treatments at T2 (Supplementary Table 9). Another eleven out of 49 DEGs responded faster to the Pieris pre-treatment than to the Botrytis pre-treatment, being found at T1 upon Pieris pre-treatment, but at T2 after inoculation by Botrytis. The 11 genes exhibited the same expression at early time point but an opposite transcriptional output at later time points. One of the gene, ERF6, was exhibited redundant function as ERF5 in response to Botrytis infection (Moffat et al., 2012). However, the scenario is different in the sequential drought condition. The ERF5 and ERF6 were down-regulated by Botrytis pre-treatment at T2 during sequential drought, but gene ERF6 exhibited enhanced down-regulation at T3 and this was not found for ERF5. ERF5 and 
ERF6 were up-regulated under Botrytis treatment and were down-regulated drought (Coolen et al., 2016)(Chapter 5). The down-regulation of the two genes in the sequential drought condition indicates an negative interaction effects between Botrytis and drought on ERF5 and ERF6, and the effect on ERF6 is stronger than on ERF5. To Pieris pre-treatment response, up-regulation of the two genes in the Pieris and subsequent drought condition was observed at T2, but only ERF6 exhibited down-regulation at T1. This is showing a negative influence of the Pieris pre-treatment on ERF6 but not on ERF5.

In total 18 out of the 49 DEGs exhibited faster response to the Pieris pretreatment than to the Botrytis pre-treatment, showing a higher impact of the Pieris rapae on transcriptome during the sequential drought. This could be due to the severity of the Pieris herbivory was much stronger than $24 \mathrm{~h}$ Botrytis treatment. Windram et al (2012) used the same concentration of Botrytis spores as in this study, and showed that the first symptom of Botrytis infection on leaf can be observed at $20 \mathrm{~h}$ after inoculation (HAI) and the expansion of lesion was at $36 \mathrm{HAI}$.

\section{Combinatorial stress responses cannot be predicted by comparing single stress responses}

Previous studies have suggested tailored responses of plants to simultaneous combinations of biotic and abiotic stresses (Prasch and Sonnewald, 2013; Ramegowda and Senthil-Kumar, 2015), which is the combinatorial stress responses cannot be predicted by comparing single stress responses. Our experimental conditions and protocols are the same as described by Coolen et al;, (2016) (Chapter 5). Thus, our data set for combinatorial stress responses were compared with the results obtained under single stresses as by Coolen et al;, (2016) (Chapter 5). When comparing DEGs from the stress combination of Botrytis and subsequent drought with single drought and Botrytis treatments (Coolen et al., 2016)(Chapter 5), only 4\% of DEGs identified as Botrytis and subsequent drought responsive genes were not induced by either single Botrytis or drought treatments (Supplementary Table 11). When comparing DEGs from the stress combination of Pieris and subsequent drought with single drought and Pieris treatments, only $3 \%$ of DEGs identified as Pieris + drought responsive 
genes were not induced by either Pieris or drought. This is showing that most combinatorial stress responsive genes can be identified by comparing results from single stresses. Genes such as ERF5 and ERF104 are the two combinatorial stress responsive genes and can be identified by comparing results from single stresses. ERF5 was up-regulated under both Botrytis and Pieris treatments, and down-regulated in drought condition. In combinatorial stress conditions, the gene was down-regulated under Botrytis and subsequent drought stress and upregulated under Pieris and subsequent drought stress. ERF104 is up-regulated under Botrytis, Pieris and drought conditions, but was down-regulated under the Botrytis and subsequent drought stress and was up-regulated under the Pieris and subsequent drought stress. This is showing that the effects of the interaction between biotic and subsequent drought responses cannot be predicted from single stress responses.

\section{PIN7-regulated auxin is essential for plants responding to Pieris attack and drought stress}

A number of alternative splicing (AS) variants were identified from each combinatorial treatment. No observed genes exhibited AS variants under both combinatorial treatments, indicating that the effects of the two biotic pretreatments on alternative RNA splicing in sequential drought conditions were different. In addition, DEGs were compared from the combinatorial treatment with genes carrying AS variants and as expected no overlap was found. This indicates differential regulation of combinatorial stress in gene expression and alternative RNA splicing. The alternative splicing of a gene causes different isoforms and the isoform expression levels can be different depending on the condition, but this does necessarily need to have an effect on the total mRNA transcripts levels. Although a small overlap between DEGs and DEGs with AS variants were observed under salt stress (Ding et al., 2014), the overrepresented function of the DEGs and the DEGs with AS variants fell into two different categories, showing a separate regulation of the two set of genes in response to stress.

The relative expression of two AS variants of auxin efflux carrier PIN7 were measured and showed enhanced expression of AS variant PIN7-1 under the 
stress combination of Pieris and subsequent drought, when compared to the single drought treatment. This shows that the PIN7-1 could play a role in response to the combinatorial stress. The two isoforms of PIN7 could have distinct biological functions, as their protein sequence is slightly different. Analysis of AS variants of the ZINC-INDUCED FACILITATOR1 (ZIFL1) gene showed that two of its isoforms (ZIFL1.1 and ZIFL1.3) exhibited distinct localization and played different roles in root tonoplast cells to modulate root polar auxin transport (ZIFL1.1) and in plasma membrane of stomatal guard cells for drought tolerance (ZIFL1.3) (Eckardt, 2013). The same AS variants of PIN7 we describe for $A$. thaliana were deposited in GenBank for the Brassicaceae Camelina sativa, though these variants had not yet been reported for $A$. thaliana, according to TAIR (www.arabidopsis.org). The biological function and the targets of the two PIN7 variants in response to stresses remains to be determined.

\section{Role of PIN7, MDAR3, and CCR2 in response to stresses}

The biological function of the combinatorial stress responsive genes (PIN7, MDAR3, and CCR2) were validated using T-DNA mutants. The PIN7 gene encodes an auxin efflux carrier expressed in the vascular tissues and columella in the mature lateral root and primary root, leaf primordia, and hypocotyl (Scarpella et al., 2006; Guyomarc'h et al., 2012; Sassi et al., 2012; Lavenus et al.). The knockout mutant pin7 exhibited decreased number of lateral root in comparison to the wild type (Lewis et al., 2011), which could be the reason for the enhanced susceptibility to drought stress. Pieris uses glucosinolate as feeding and oviposition stimulus (de Vos et al., 2008). Thus, one of our hypothesis for the decreased leaf consumption by Pieris is due to less indole-3carbinol (I3C) accumulation in pin7 leafs. However, an antagonistic interaction has been suggested between the glucosinolate breakdown products I3C and auxin (Katz et al., 2015). Another hypothesis for the enhanced tolerance to Pieris in pin 7 could be due to the negative regulation between auxin and $\mathrm{JA}$ biosynthesis (Cecchetti et al., 2013). The pin7 mutant could contain higher JA accumulation than the wild type and that regulates Pieris defence genes expression. The MDAR3 gene is a member of the monodehydroascorbate reductase gene family and plays an important role in recapturing ascorbic acid (AsA) and regulates redox homeostasis in plant cell (Ramel et al., 2009; Brini et 
al., 2011). The MDAR3 expression is regulated by ERF6 and the expression of MADAR3 can be inhibited by ABA and heat treatments (Sewelam et al., 2013), as well as drought stress (this study). The AsA level is decreased under drought because AsA has impair on ABA- and $\mathrm{H}_{2} \mathrm{O}_{2}$-mediated stomatal closing (Pastori and Foyer, 2002). Inhibit MDAR3 expression reduces AsA level in plants cell and results increased tolerance to drought stress by closing stomata. The CCR2 gene encodes one of cinnamoyl CoA reductase (CCR) isoforms and is involved in lignification and phenolic biosynthesis (Lauvergeat et al., 2001). The lignification is inovlved in response to multiple biotic and abiotic stresses. For instance, The phenolic acids can affect Pieris oviposition and feeding stimulants (van Loon, 1990), the lignification and CCR proteins were increased drought and salt stress in Leucaena leucocephala(Srivastava et al., 2015). A study in maize showed that the transcript level of CCR2 is increased under drought stress and the increased expression of CCR2 results decreased cell wall expansion, increased deposition of lignin, and reduced root elongation (Fan et al., 2006). The ccr2 mutant could promote root elongation and that enhances the sensitivity of ccr2 mutant to drought stress. 


\section{Conclusion}

In this paper, we have shown the gene expression and alternative splicing of genes that are associated with drought and two combinatorial pre-treatment stress responses in Arabidopsis. An increased number of DEGs across time points under drought stress were identified, showing an increased severity of drought experienced by plants. We found that one-day rehydration period is insufficient to fully overcome the effects of drought stress on Arabidopsis. More DEGs under the Pieris and subsequent drought stress were identified than under the Botrytis on subsequent drought stress, showing that the Pieris herbivory is more severe than Botrytis infection. A small set of DEGs were shared by the two combinatorial stresses and most of the genes showed opposite transcriptional output in the two combinatorial conditions, indicating different regulations of the two combinatorial stress in gene expression. We validated some combinatorial stress responsive genes using loss-of-function mutants and observed antagonistic interaction between Pieris and drought. Some unique combinatorial stress responsive genes were discovered, but their biological function in stress conditions still remains unresolved. We confirmed that the expression level of alternative splicing variant PIN7-1 changes under drought and the combination of Pieris and subsequent drought, but further research will be needed to establish if and how this affects the phenotype. 


\section{Acknowledgement}

We gratefully acknowledge the help of Kaige Zhao, Hans van Pelt, Michel Arts, Kim Vermeer, Elena Kapsomenou, Benno Augustinus, Robert Veldman and Tom van den Beuken, with collecting the samples. Raymond Hulzink and Roy Gorkink (KeyGene) kindly performed the RNA-seq and the initial data quality control. We thank Maarten Koornneef and Robert Akkers for critical reading of the manuscript and numerous very useful suggestions. This work was financially supported by The Netherlands Organization for Scientific Research (NWO) through the Technology Foundation, Perspective Programme 'Learning from Nature' [STW10988]. 


\section{References}

Achuo EA, Prinsen E, Höfte M (2006) Influence of drought, salt stress and abscisic acid on the resistance of tomato to Botrytis cinerea and Oidium neolycopersici Plant Pathology Volume 55, Issue 2. In Plant Pathology, Vol 55, pp 178-186

Adamczyk BJ, Lehti-Shiu MD, Fernandez DE (2007) The MADS domain factors AGL15 and AGL18 act redundantly as repressors of the floral transition in Arabidopsis. Plant J 50: 1007-1019

Anders S, Huber W (2010) Differential expression analysis for sequence count data. Genome Biol 11: R106

Anders S, PyI PT, Huber W (2015) HTSeq--a Python framework to work with highthroughput sequencing data. Bioinformatics 31: 166-169

Anderson JP, Badruzsaufari E, Schenk PM, Manners JM, Desmond OJ, Ehlert C, Maclean DJ, Ebert PR, Kazan K (2004) Antagonistic interaction between abscisic acid and jasmonate-ethylene signaling pathways modulates defense gene expression and disease resistance in Arabidopsis. Plant Cell 16: 3460-3479

Asai T, Tena G, Plotnikova J, Willmann MR, Chiu WL, Gomez-Gomez L, Boller T, Ausubel FM, Sheen J (2002) MAP kinase signalling cascade in Arabidopsis innate immunity. Nature 415: 977-983

Atkinson NJ, Urwin PE (2012) The interaction of plant biotic and abiotic stresses: from genes to the field. J Exp Bot 63: 3523-3543

Audenaert K, De Meyer GB, Hofte MM (2002) Abscisic acid determines basal susceptibility of tomato to Botrytis cinerea and suppresses salicylic acid-dependent signaling mechanisms. Plant Physiol 128: 491-501

Bethke G, Unthan T, Uhrig JF, PoschI Y, Gust AA, Scheel D, Lee J (2009) Flg22 regulates the release of an ethylene response factor substrate from MAP kinase 6 in Arabidopsis thaliana via ethylene signaling. Proc Natl Acad Sci U S A 106: 8067-8072

Brini F, Yamamoto A, Jlaiel L, Takeda S, Hobo T, Dinh HQ, Hattori T, Masmoudi K, Hanin M (2011) Pleiotropic effects of the wheat dehydrin DHN-5 on stress responses in Arabidopsis. Plant Cell Physiol 52: 676-688

Bueso E, Rodriguez L, Lorenzo-Orts L, Gonzalez-Guzman M, Sayas E, MunozBertomeu J, Ibanez C, Serrano R, Rodriguez PL (2014) The single-subunit RING-type E3 ubiquitin ligase RSL1 targets PYL4 and PYR1 ABA receptors in plasma membrane to modulate abscisic acid signaling. Plant J 80: 1057-1071

Cecchetti V, Altamura MM, Brunetti P, Petrocelli V, Falasca G, Ljung $K$, Costantino P, Cardarelli M (2013) Auxin controls Arabidopsis anther dehiscence by regulating endothecium lignification and jasmonic acid biosynthesis. Plant J 74: 411-422

Chen YE, Liu WJ, Su YQ, Cui JM, Zhang ZW, Yuan M, Zhang HY, Yuan S (2016) Different response of photosystem II to short and long term drought stress in Arabidopsis thaliana. Physiol Plant

Chini A, Fonseca S, Fernandez G, Adie B, Chico JM, Lorenzo O, Garcia-Casado G, Lopez-Vidriero I, Lozano FM, Ponce MR, Micol JL, Solano R (2007) The JAZ family of repressors is the missing link in jasmonate signalling. 448: 666-671

Coolen S, Proietti S, Hickman R, Davila Olivas NH, Huang PP, Van Verk MC, Van Pelt JA, Wittenberg AH, De Vos M, Prins M, Van Loon JJ, Aarts MGM, Dicke M, Pieterse CM, Van Wees SC (2016) Transcriptome dynamics of Arabidopsis during sequential biotic and abiotic stresses. Plant J

Davila Olivas NH, Coolen S, Huang P, Severing E, van Verk MC, Hickman R, Wittenberg AH, de Vos M, Prins M, van Loon JJ, Aarts MGM, van Wees SC, Pieterse CM, Dicke M (2016) Effect of prior drought and pathogen stress on Arabidopsis transcriptome changes to caterpillar herbivory. New Phytol 210: 1344-1356

de Souza A, Hull PA, Gille S, Pauly M (2014) Identification and functional characterization of the distinct plant pectin esterases PAE8 and PAE9 and their deletion mutants. Planta 240: 1123-1138

de Vos M, Kriksunov KL, Jander G (2008) Indole-3-acetonitrile production from indole glucosinolates deters oviposition by Pieris rapae. Plant Physiol 146: 916-926 
Ding F, Cui P, Wang Z, Zhang S, Ali S, Xiong L (2014) Genome-wide analysis of alternative splicing of pre-mRNA under salt stress in Arabidopsis. BMC Genomics 15: 431

Ding Y, Shaholli D, Mou Z (2014) A large-scale genetic screen for mutants with altered salicylic acid accumulation in Arabidopsis. Front Plant Sci 5: 763

Ding ZJ, Yan JY, Xu XY, Yu DQ, Li GX, Zhang SQ, Zheng SJ (2014) Transcription factor WRKY46 regulates osmotic stress responses and stomatal movement independently in Arabidopsis. Plant J 79: 13-27

Dodds PN, Rathjen JP (2010) Plant immunity: towards an integrated view of plantpathogen interactions. Nat Rev Genet 11: 539-548

Dombrecht B, Xue GP, Sprague SJ, Kirkegaard JA, Ross JJ, Reid JB, Fitt GP, Sewelam N, Schenk PM, Manners JM, Kazan K (2007) MYC2 differentially modulates diverse jasmonate-dependent functions in Arabidopsis. Plant Cell 19: 2225-2245

Eckardt NA (2013) Alternative splicing confers a dual role in polar auxin transport and drought stress tolerance to the major facilitator superfamily transporter ZIFL1. Plant Cell 25: 779

Fan L, Linker R, Gepstein S, Tanimoto E, Yamamoto R, Neumann PM (2006) Progressive inhibition by water deficit of cell wall extensibility and growth along the elongation zone of maize roots is related to increased lignin metabolism and progressive stelar accumulation of wall phenolics. Plant Physiol 140: 603-612

Flexas J, Medrano H (2002) Drought-inhibition of photosynthesis in C3 plants: stomatal and non-stomatal limitations revisited. Ann Bot 89: 183-189

Guyomarc'h S, Leran S, Auzon-Cape M, Perrine-Walker F, Lucas M, Laplaze L (2012) Early development and gravitropic response of lateral roots in Arabidopsis thaliana. Philos Trans R Soc Lond B Biol Sci 367: 1509-1516

Hirt H (2009) Plant Stress Biology: From Genomics to Systems Biology. Wiley

Hirt H, Shinozaki K (2004) Plant Responses to Abiotic Stress. Springer

Humbert S, Zhong S, Deng Y, Howell SH, Rothstein SJ (2012) Alteration of the bZIP60/IRE1 pathway affects plant response to ER stress in Arabidopsis thaliana. PLoS One 7: e39023

Hunt E, Gattolin S, Newbury HJ, Bale JS, Tseng HM, Barrett DA, Pritchard J (2010) A mutation in amino acid permease AAP6 reduces the amino acid content of the Arabidopsis sieve elements but leaves aphid herbivores unaffected. J Exp Bot 61: 55-64

Jansson S (1994) The light-harvesting chlorophyll ab-binding proteins. Biochimica et Biophysica Acta (BBA) - Bioenergetics 1184: 1-19

Katz E, Nisani S, Sela M, Behar H, Chamovitz DA (2015) The effect of indole-3carbinol on PIN1 and PIN2 in Arabidopsis roots. Plant Signal Behav 10: e1062200

Katz E, Nisani S, Yadav BS, Woldemariam MG, Shai B, Obolski U, Ehrlich M, Shani E, Jander G, Chamovitz DA (2015) The glucosinolate breakdown product indole3-carbinol acts as an auxin antagonist in roots of Arabidopsis thaliana. Plant J 82: 547-555

Katz Y, Wang ET, Airoldi EM, Burge CB (2010) Analysis and design of RNA sequencing experiments for identifying isoform regulation. Nat Methods 7: 1009-1015

Kim J, Malladi A, van Iersel MW (2012) Physiological and molecular responses to drought in Petunia: the importance of stress severity. J Exp Bot 63: 6335-6345

Kim SH, Woo DH, Kim JM, Lee SY, Chung WS, Moon YH (2011) Arabidopsis MKK4 mediates osmotic-stress response via its regulation of MPK3 activity. Biochem Biophys Res Commun 412: 150-154

Kiyosue T, Abe H, Yamaguchi-Shinozaki K, Shinozaki K (1998) ERD6, a cDNA clone for an early dehydration-induced gene of Arabidopsis, encodes a putative sugar transporter1. Biochimica et Biophysica Acta (BBA) - Biomembranes 1370: 187-191

Lackman P, Gonzalez-Guzman M, Tilleman S, Carqueijeiro I, Perez AC, Moses T, Seo M, Kanno Y, Hakkinen ST, Van Montagu MC, Thevelein JM, Maaheimo H, Oksman-Caldentey KM, Rodriguez PL, Rischer H, Goossens A (2011) Jasmonate signaling involves the abscisic acid receptor PYL4 to regulate metabolic reprogramming in Arabidopsis and tobacco. Proc Natl Acad Sci U S A 108: 5891-5896

Lauvergeat V, Lacomme C, Lacombe E, Lasserre E, Roby D, Grima-Pettenati J (2001) Two cinnamoyl-CoA reductase (CCR) genes from Arabidopsis thaliana are 
differentially expressed during development and in response to infection with pathogenic bacteria. Phytochemistry 57: 1187-1195

Lavenus J, Guyomarc'h S, Laplaze L (2016) PIN Transcriptional Regulation Shapes Root System Architecture. Trends in Plant Science 21: 175-177

Lewis DR, Negi S, Sukumar P, Muday GK (2011) Ethylene inhibits lateral root development, increases IAA transport and expression of PIN3 and PIN7 auxin efflux carriers. Development 138: 3485-3495

Li S, Strid A (2005) Anthocyanin accumulation and changes in CHS and PR-5 gene expression in Arabidopsis thaliana after removal of the inflorescence stem (decapitation). Plant Physiol Biochem 43: 521-525

Litholdo CG, Jr., Parker BL, Eamens AL, Larsen MR, Cordwell SJ, Waterhouse PM (2016) Proteomic Identification of Putative MicroRNA394 Target Genes in Arabidopsis thaliana Identifies Major Latex Protein Family Members Critical for Normal Development. Mol Cell Proteomics 15: 2033-2047

Long TA, Tsukagoshi H, Busch W, Lahner B, Salt DE, Benfey PN (2010) The bHLH transcription factor POPEYE regulates response to iron deficiency in Arabidopsis roots. Plant Cell 22: 2219-2236

Love MI, Huber W, Anders S (2014) Moderated estimation of fold change and dispersion for RNA-seq data with DESeq2. Genome Biol 15: 550

Marella HH, Nielsen E, Schachtman DP, Taylor CG (2013) The amino acid permeases AAP3 and AAP6 are involved in root-knot nematode parasitism of Arabidopsis. Mol Plant Microbe Interact 26: 44-54

Mizoi J, Shinozaki K, Yamaguchi-Shinozaki K (2012) AP2/ERF family transcription factors in plant abiotic stress responses. Biochim Biophys Acta 1819: 86-96

Moffat CS, Ingle RA, Wathugala DL, Saunders NJ, Knight H, Knight MR (2012) ERF5 and ERF6 Play Redundant Roles as Positive Regulators of JA/Et-Mediated Defense against <italic> Botrytis cinerea</italic> in Arabidopsis. PLoS ONE 7: e35995

Moffat CS, Ingle RA, Wathugala DL, Saunders NJ, Knight H, Knight MR (2012) ERF5 and ERF6 play redundant roles as positive regulators of JA/Et-mediated defense against Botrytis cinerea in Arabidopsis. PLoS One 7: e35995

Moore JP, Vicre-Gibouin M, Farrant JM, Driouich A (2008) Adaptations of higher plant cell walls to water loss: drought vs desiccation. Physiol Plant 134: 237-245

Narsai R, Wang C, Chen J, Wu J, Shou H, Whelan J (2013) Antagonistic, overlapping and distinct responses to biotic stress in rice (Oryza sativa) and interactions with abiotic stress. BMC Genomics 14: 93

Osterlund MT, Hardtke CS, Wei N, Deng XW (2000) Targeted destabilization of HY5 during light-regulated development of Arabidopsis. Nature 405: 462-466

Pareek A, Sopory SK, Bohnert H (2010) Abiotic Stress Adaptation in Plants: Physiological, Molecular and Genomic Foundation. Springer Netherlands

Pastori GM, Foyer CH (2002) Common Components, Networks, and Pathways of Cross-Tolerance to Stress. The Central Role of "Redox" and Abscisic Acid-Mediated Controls. Plant Physiology 129: 460-468

Pieterse CM, Leon-Reyes A, Van der Ent S, Van Wees SC (2009) Networking by small-molecule hormones in plant immunity. Nat Chem Biol 5: 308-316

Pieterse CM, Van der Does D, Zamioudis C, Leon-Reyes A, Van Wees SC (2012) Hormonal modulation of plant immunity. Annu Rev Cell Dev Biol 28: 489-521

Prasch CM, Sonnewald U (2013) Simultaneous application of heat, drought, and virus to Arabidopsis plants reveals significant shifts in signaling networks. Plant Physiol 162: $1849-1866$

Prasch CM, Sonnewald U (2015) Signaling events in plants: Stress factors in combination change the picture. Environmental and Experimental Botany 114: 4-14

Pre M, Atallah M, Champion A, De Vos M, Pieterse CM, Memelink J (2008) The AP2/ERF domain transcription factor ORA59 integrates jasmonic acid and ethylene signals in plant defense. Plant Physiol 147: 1347-1357

Pré M, Atallah M, Champion A, De Vos M, Pieterse CMJ, Memelink J (2008) The AP2/ERF Domain Transcription Factor ORA59 Integrates Jasmonic Acid and Ethylene Signals in Plant Defense. Plant Physiology 147: 1347-1357 
Ramegowda V, Senthil-Kumar M (2015) The interactive effects of simultaneous biotic and abiotic stresses on plants: mechanistic understanding from drought and pathogen combination. J Plant Physiol 176: 47-54

Ramel F, Sulmon C, Bogard M, Couée I, Gouesbet G (2009) Differential patterns of reactive oxygen species and antioxidative mechanisms during atrazine injury and sucrose-induced tolerance in Arabidopsis thaliana plantlets. BMC Plant Biology 9: 1-18

Sakuma Y, Maruyama K, Osakabe Y, Qin F, Seki M, Shinozaki K, YamaguchiShinozaki K (2006) Functional analysis of an Arabidopsis transcription factor, DREB2A, involved in drought-responsive gene expression. Plant Cell 18: 1292-1309

Santiago J, Rodrigues A, Saez A, Rubio S, Antoni R, Dupeux F, Park SY, Marquez JA, Cutler SR, Rodriguez PL (2009) Modulation of drought resistance by the abscisic acid receptor PYL5 through inhibition of clade A PP2Cs. Plant J 60: 575-588

Sassi M, Lu Y, Zhang Y, Wang J, Dhonukshe P, Blilou I, Dai M, Li J, Gong X, Jaillais Y, Yu X, Traas J, Ruberti I, Wang H, Scheres B, Vernoux T, Xu J (2012) COP1 mediates the coordination of root and shoot growth by light through modulation of PIN1- and PIN2-dependent auxin transport in Arabidopsis. Development 139: 3402-3412

Scarpella E, Marcos D, Friml J, Berleth T (2006) Control of leaf vascular patterning by polar auxin transport. Genes Dev 20: 1015-1027

Sewelam N, Kazan K, Thomas-Hall SR, Kidd BN, Manners JM, Schenk PM (2013) Ethylene response factor 6 is a regulator of reactive oxygen species signaling in Arabidopsis. PLoS One 8: e70289

Sewelam N, Oshima Y, Mitsuda N, Ohme-Takagi M (2014) A step towards understanding plant responses to multiple environmental stresses: a genome-wide study. Plant Cell Environ 37: 2024-2035

Shaik R, Ramakrishna W (2013) Genes and co-expression modules common to drought and bacterial stress responses in Arabidopsis and rice. PLoS One 8: e77261

Shinozaki K, Yamaguchi-Shinozaki K (2007) Gene networks involved in drought stress response and tolerance. J Exp Bot 58: 221-227

Shukla D, Tiwari M, Tripathi RD, Nath P, Trivedi PK (2013) Synthetic phytochelatins complement a phytochelatin-deficient Arabidopsis mutant and enhance the accumulation of heavy metal(loid)s. Biochem Biophys Res Commun 434: 664-669

Sistrunk ML, Antosiewicz DM, Purugganan MM, Braam J (1994) Arabidopsis $\mathrm{TCH} 3$ encodes a novel $\mathrm{Ca} 2+$ binding protein and shows environmentally induced and tissue-specific regulation. Plant Cell 6: 1553-1565

Smékalová V, Doskočilová A, Komis G, Šamaj J (2014) Crosstalk between secondary messengers, hormones and MAPK modules during abiotic stress signalling in plants. Biotechnology Advances 32: 2-11

Srivastava S, Vishwakarma RK, Arafat YA, Gupta SK, Khan BM (2015) Abiotic stress induces change in Cinnamoyl CoA Reductase (CCR) protein abundance and lignin deposition in developing seedlings of Leucaena leucocephala. Physiol Mol Biol Plants 21: 197-205

Staats M, Van Kan JAL (2012) Genome update of Botrytis cinerea strains B05.10 and T4. Eukaryotic Cell 11: 1413-1414

Staiger D, Brown JWS (2013) Alternative Splicing at the Intersection of Biological Timing, Development, and Stress Responses. The Plant Cell 25: 3640-3656

Székely G, Ábrahám E, Csépl Á, Rigó G, Zsigmond L, Csiszár J, Ayaydin F, Strizhov N, Jásik J, Schmelzer E, Koncz C, Szabados L (2008) Duplicated P5CS genes of Arabidopsis play distinct roles in stress regulation and developmental control of proline biosynthesis The Plant Journal Volume 53, Issue 1. In The Plant Journal, Vol 53, pp 11-28

Taji T, Seki M, Yamaguchi-Shinozaki K, Kamada H, Giraudat J, Shinozaki K (1999) Mapping of 25 drought-inducible genes, RD and ERD, in Arabidopsis thaliana. Plant Cell Physiol 40: 119-123

Takahashi F, Mizoguchi T, Yoshida R, Ichimura K, Shinozaki K (2011) Calmodulin-dependent activation of MAP kinase for ROS homeostasis in Arabidopsis. Mol Cell 41: 649-660

Trapnell C, Pachter L, Salzberg SL (2009) TopHat: discovering splice junctions with RNA-Seq. Bioinformatics 25: 1105-1111 
Trapnell C, Roberts A, Goff L, Pertea G, Kim D, Kelley DR, Pimentel H, Salzberg SL, Rinn JL, Pachter L (2012) Differential gene and transcript expression analysis of RNA-seq experiments with TopHat and Cufflinks. Nat Protoc 7: 562-578

Tuteja N, Sarvajeet SG (2012) Plant Acclimation to Environmental Stress. Springer New York

van Loon JJA (1990) Chemoreception of phenolic acids and flavonoids in larvae of two species of Pieris. Journal of Comparative Physiology A 166: 889-899

Van Wees SC, Van Pelt JA, Bakker PA, Pieterse CM (2013) Bioassays for assessing jasmonate-dependent defenses triggered by pathogens, herbivorous insects, or beneficial rhizobacteria. Methods Mol Biol 1011: 35-49

Van Wees SCM, Van Pelt JA, Bakker PAHM, Pieterse CMJ (2013) Bioassays for assessing jasmonate-dependent defenses triggered by pathogens, herbivorous Insects, or beneficial rhizobacteria. Methods in Molecular Biology 1011: 35-49

Vos IA, Verhage A, Schuurink RC, Watt LG, Pieterse CMJ, Van Wees SCM (2013) Onset of herbivore-induced resistance in systemic tissue primed for jasmonatedependent defenses is activated by abscisic acid. Frontiers in Plant Science 4: 539

Werner T, Motyka V, Laucou V, Smets R, Van Onckelen H, Schmülling T (2003) Cytokinin-Deficient Transgenic Arabidopsis Plants Show Multiple Developmental Alterations Indicating Opposite Functions of Cytokinins in the Regulation of Shoot and Root Meristem Activity. The Plant Cell 15: 2532-2550

Windram O, Madhou P, McHattie S, Hill C, Hickman R, Cooke E, Jenkins DJ, Penfold CA, Baxter L, Breeze E, Kiddle SJ, Rhodes J, Atwell S, Kliebenstein DJ, Kim YS, Stegle O, Borgwardt K, Zhang C, Tabrett A, Legaie R, Moore J, Finkenstadt B, Wild DL, Mead A, Rand D, Beynon J, Ott S, Buchanan-Wollaston V, Denby KJ (2012) Arabidopsis defense against Botrytis cinerea: chronology and regulation deciphered by high-resolution temporal transcriptomic analysis. Plant Cell 24: 3530-3557

Xie Z, Li D, Wang L, Sack FD, Grotewold E (2010) Role of the stomatal development regulators FLP/MYB88 in abiotic stress responses. The Plant Journal 64: 731739

Xing Y, Chen WH, Jia W, Zhang J (2015) Mitogen-activated protein kinase kinase 5 (MKK5)-mediated signalling cascade regulates expression of iron superoxide dismutase gene in Arabidopsis under salinity stress. J Exp Bot 66: 5971-5981

Xu P, Chen F, Mannas JP, Feldman T, Sumner LW, Roossinck MJ (2008) Virus infection improves drought tolerance. New Phytol 180: 911-921

Xu YH, Liu R, Yan L, Liu ZQ, Jiang SC, Shen YY, Wang XF, Zhang DP (2012) Light-harvesting chlorophyll a/b-binding proteins are required for stomatal response to abscisic acid in Arabidopsis. J Exp Bot 63: 1095-1106

Yamburenko MV, Zubo YO, Borner T (2015) Abscisic acid affects transcription of chloroplast genes via protein phosphatase 2C-dependent activation of nuclear genes: repression by guanosine-3'-5'-bisdiphosphate and activation by sigma factor 5 . Plant $\mathrm{J} \mathbf{8 2}$ : 1030-1041

Yang Y, Xu R, Ma C-j, Vlot AC, Klessig DF, Pichersky E (2008) Inactive Methyl Indole-3-Acetic Acid Ester Can Be Hydrolyzed and Activated by Several Esterases Belonging to the AtMES Esterase Family of Arabidopsis. Plant Physiology 147: 1034-1045

Yang Z, Tian L, Latoszek-Green M, Brown D, Wu K (2005) Arabidopsis ERF4 is a transcriptional repressor capable of modulating ethylene and abscisic acid responses. Plant Mol Biol 58: 585-596

Yi X, Du Z, Su Z (2013) PlantGSEA: a gene set enrichment analysis toolkit for plant community. Nucleic Acids Res 41: W98-103

Yoshida R, Hobo T, Ichimura K, Mizoguchi T, Takahashi F, Aronso J, Ecker JR, Shinozaki K (2002) ABA-activated SnRK2 protein kinase is required for dehydration stress signaling in Arabidopsis. Plant Cell Physiol 43: 1473-1483

Yoshioka K, Shinozaki K (2009) Signal Crosstalk in Plant Stress Responses. Wiley

Zheng Z, Qamar SA, Chen Z, Mengiste T (2006) Arabidopsis WRKY33 transcription factor is required for resistance to necrotrophic fungal pathogens. Plant J 48: 592-605

Zou J-J, Li X-D, Ratnasekera D, Wang C, Liu W-X, Song L-F, Zhang W-Z, Wu W-H (2015) Arabidopsis CALCIUM-DEPENDENT PROTEIN KINASE8 and CATALASE3 
Function in Abscisic Acid-Mediated Signaling and $\mathrm{H}(2) \mathrm{O}(2)$ Homeostasis in Stomatal Guard Cells under Drought Stress. The Plant Cell 27: 1445-1460 


\section{Supporting Information}

Additional supporting information may be download via link

https://www.dropbox.com/sh/hb95t5dwvxbsc1u/AAB2Z7Q1DPSwCCUoaTBBi2Pa?dl=0 



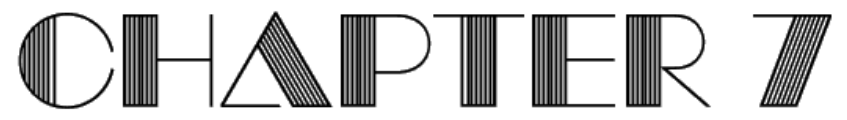

Phenotypic and genetic responses of Arabidopsis thaliana natural accessions to consecutive Pieris rapae herbivory and drought stress

Pingping Huang, Michael Mintgen, Mark G.M. Aarts

Laboratory of Genetics, Wageningen University, Droevendaalsesteeg 1, 6708 PB, Wageningen, the Netherlands 


\section{Abstract}

This study focuses on investigating the responses of natural accessions of Arabidopsis thaliana to the consecutive exposure to a biotic agent (herbivory by Pieris rapae) and an abiotic factor (drought) as well as to each of the single treatments. Three parameters: rosette fresh weight (FW), dry weight (DW), and water content (WC) were used to evaluate the effect of exposure to Pieris and/or subsequent drought stress in 16 Arabidopsis accessions. We found WC is a suitable parameter for the Pieris and drought stress interaction assessment and showed it to be independent of leaf biomass accumulation under drought conditions. To gauge the effect of Pieris pre-treatment on sequential drought response in planta, differential expression levels of nine drought-responsive genes, relevant to the cross-talk between Abscisic acid (ABA) and jasmonic acid (JA) signaling pathways, were measured. While no correlation with the observed phenotypic variation was found, the expression of all investigated gene candidates (except $R D 29 b$ ) proved affected in response to drought preceded by exposure to the herbivore activity. Results presented herein allowed us to propose a model of gene regulatory networks effectively delineating the effects of the interaction between Pieris and drought stress triggers on droughtresponsive gene expression.

\section{Keywords}

Arabidopsis thaliana, (a)biotic stress, consecutive stress combination, drought-responsive gene expression 


\section{Introduction}

Biotic and abiotic stress factors, such as pathogen attack, insect herbivory, drought, extreme temperatures, UV radiation, salinity, etc. (Pareek et al., 2010; Robert-Seilaniantz et al., 2010) often exert negative effects on plant growth and development causing immense losses in agricultural production (Hirt, 2004). Drought is one of the most devastating abiotic stress elicitors in plants, resulting in accumulation of abscisic acid ( $A B A)$, a phytohormone triggering stomatal closure to reduce water loss via transpiration (Rai and Takabe, 2006; Xiong and Ishitani, 2006). As a consequence, water uptake and assimilation are reduced, leading to lower nutrient uptake and diminished biomass (Verslues et al., 2006).

The ABA-dependent signaling pathway has been reported crucial in mediation of plant stress-responsive gene expression under drought condition, while also being involved in osmotic and pathogen stress response mechanisms (Rai and Takabe, 2006; Xiong and Ishitani, 2006). A comprehensive overview of ABA-dependent and ABA-independent signaling trails in planta as triggered by drought stress was proposed by Shinozaki and Yamaguchi-Shinozaki (2007). Zeaxanthin epoxidase, catalyzing the first committed step in the $A B A$ biosynthetic pathway, is encoded by ABA DEFICIENT 1 (ABA1) (Xiong and Zhu, 2003), whose expression is enhanced in response to drought as well as osmotic stress, high salinity, and ABA treatment (Xiong et al., 2002; Barrero et al., 2006) and down-regulated by JA (Anderson et al., 2004). Furthermore, multiple transcription factors (TFs), such as RESPONSIVE TO DESICCATION 26 (RD26), MYB2, MYC2, RD22, and ABSCISIC ACID RESPONSIVE ELEMENTS-BINDING FACTOR 2 (ABF2), RESPONSIVE TO DESICCATION 29B (RD29b) participate in the $A B A-d e p e n d e n t$ pathway and are involved in the cross-talk between $A B A$ and jasmonic acid (JA) signaling (Shinozaki and Yamaguchi-Shinozaki, 2007). Their genes are expressed in response to multiple biotic and abiotic triggers, including drought, high salinity, osmotic stress, Fusarium oxysporum infection, and herbivory by Pieris rapae (Abe et al., 2003; Anderson et al., 2004; Fujita et al., 2004; Shinozaki and Yamaguchi-Shinozaki, 2007; Vos et al., 2013). Among the aforementioned TFs, MYC2, a positive regulator of Pieris defense as well as drought tolerance (Vos et al., 2013), is of special significance. Transgenic 
overexpression of $M Y B 2$ and $M Y C 2$ resulted in plants exhibiting enhanced sensitivity to ABA treatment, with the MYB2 transformants proving less responsive to the phytohormone in comparison with their MYC2 overexpressing counterparts (Abe et al., 2003). Further, the promoter of RD22 contains two ciselements that can bind respectively with MYB2 and MYC2 (Abe et al., 2003), while gene expression of RD29b is regulated by ABF2, as well as ANAC019 in response to dehydration treatment (Jensen et al., 2010; Uno et al., 2000; Yamaguchi-Shinozaki and Shinozaki, 1994). Moreover, the DEHYDRATIONRESPONSIVE ELEMENT BINDING PROTEIN 2 (DREB2a), playing a role in the ABA-independent pathway, was reported as sensitive to drought (Shinozaki and Yamaguchi-Shinozaki, 2007). Although the upstream sequence of the initial transcriptional site of $D R E B 2 a$ contains an ABA-responsive AREB element, and its expression can be activated by ABF2 (AREB1), AREB2, and AREB3, only low expression levels of the gene were observed after exposure to the plant hormone (Kim et al., 2011), while enhanced expression of DREB2a was detected after 24 hours of rehydration treatment (Yamaguchi-Shinozaki and Shinozaki, 1994). What is more, plants under drought stress were found to accumulate high levels of proline (Delauney and Verma, 1993). DELTA1-PYRROLINE-5CARBOXYLATE SYNTHASE 1 (P5CS1) is one of the proline biosynthetic genes (Strizhov et al., 1997) and is required for the build-up of the amino acid levels under osmotic, high salinity, and drought stress conditions (Szekely et al., 2008; Verslues and Sharma, 2010). The gene can be regulated via both the ABAdependent and the ABA-independent pathway (Verslues and Sharma, 2010). The enumerated drought-responsive genes are representative of the cross-talk between the $A B A$ and $J A$ cascades, as well the cross-take between ABAdependent and ABA-independent signal transduction mechanism that triggered by the abiotic stress factor in question.

Various interactions between biotic and abiotic stress response mechanisms in plants have been reported and summarized by Suzuki et al. (2014). The interplay between biotic and drought stress feedback involves complex crosstalk via both metabolic and signaling pathways (Atkinson and Urwin, 2012; Prasch and Sonnewald, 2013; Rasmussen et al., 2013). For instance, the biotic herbivory of Pieris rapae larvae induces signaling through the jasmonic acid and 
ethylene (JA-ET) cascade (De Keersmaecker et al., 2005), which can interacts negatively with the drought-induced ABA signaling (Pieterse et al., 2012). The latter observation suggests that Pieris pre-treatment might lead to increased drought susceptibility. Thus, understanding how plants adapt to the wide range of stress factors and the genetics underlying their response will undoubtedly contribute to the improvement of plant tolerance to extreme conditions via optimized breeding.

This study was designed to gain novel insights into the mechanism of plant response to combinatorial stress, namely insect attack and subsequent drought, through observation of the variation of Arabidopsis thaliana natural accessions subjected to the investigated stress factor combination. Arabidopsis plants were exposed to Pieris rapae larvae, to elicit response to herbivory, and then treated with polyethylene glycol (PEG) which induces osmotic stress simulating drought. Control plants were subjected to single stress treatments (Pieris herbivory only and single PEG treatment without any pre-treatment). Responses to the applied stress-inducing conditions were investigated using phenotypic analysis in combination with expression profiling of the aforementioned nine droughtresponsive genes. 


\section{Materials and methods}

\section{Plant material and growth conditions}

Sixteen Arabidopsis thaliana accessions that showed maximum genetic divergence from the HapMap population were selected based on single nucleotide polymorphism (SNP) marker information ( $\mathrm{Li}$ et al., 2010). These included: Br-0 (CS76101), Bur-0 (CS76105), Col-0 (CS76113), Est-1 (CS76127), Fei-0 (CS76129), Got-7 (CS76136), Ler-1 (CS76164), Lov-5 (CS76175), NFA-8 (CS76199), RRS-7 (CS28713), RRS-10 (CS22689), Sha (CS76227), Tamm-2 (CS76244), Ts-1 (CS76268), Tsu-0 (CS28780), and Van-0 (CS76297).

Prior to experimental procedure commencement, seeds of the test $A$. thaliana accessions were stratified at $4{ }^{\circ} \mathrm{C}$ in the dark for five days. Thereafter, one seed per accession was sown on one Grodan ${ }^{\circledR}$ Rockwool cube of $40 \times 40 \times 40$ $\mathrm{mm}$. The plants were watered three times per week (on Mondays, Wednesdays, and Fridays) with a nutrient solution developed for Arabidopsis ( $\mathrm{pH}, 7$; EC, 1.4 $\mathrm{mS} / \mathrm{cm}$ ). The solution consisted of $1.7 \mathrm{mM} \mathrm{NH}_{4}^{+}, 4.5 \mathrm{mM} \mathrm{K}^{+}, 0.4 \mathrm{mM} \mathrm{Na}^{+}, 2.3$ $\mathrm{mM} \mathrm{Ca2}^{+}, 1.5 \mathrm{mM} \mathrm{Mg2}^{+}, 4.4 \mathrm{mM} \mathrm{NO}_{3}{ }^{-}, 0.2 \mathrm{mM} \mathrm{Cl}^{-}, 3.5 \mathrm{mM} \mathrm{SO}_{4}{ }^{2-}, 0.6 \mathrm{mM} \mathrm{HCO}_{3}{ }^{-}$,

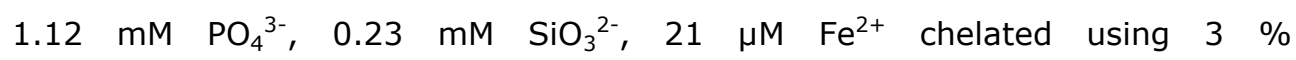

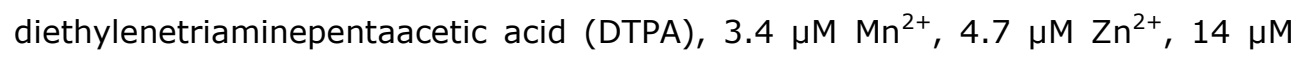
$\mathrm{BO}_{3}{ }^{3-}, 6.9 \mu \mathrm{M} \mathrm{Cu}^{2+}$, and $<0.1 \mu \mathrm{M} \mathrm{MoO}_{4}{ }^{4-}$. The plants were grown in a climatecontrolled growth chamber set to short day conditions, i.e., $10 \mathrm{~h}$ day/14 h night at $21{ }^{\circ} \mathrm{C}$ day $/ 19{ }^{\circ} \mathrm{C}$ night and $70 \%$ relative humidity. Irradiation was set to 200 $\mu \mathrm{mol} \mathrm{m} \mathrm{m}^{-2} \mathrm{~s}^{-1}$.

\section{Preparation of Pieris rapae larvae}

Pieris rapae caterpillars were reared on cabbage plants (Brassica oleracea convar. capitata var. alba) under greenhouse conditions ( $24^{\circ} \mathrm{C}$, natural daylight). Butterflies were supplied with flowering plants, such as Lantana camara, for their nourishment (nectar). When the flowers were scarce, additional food source (a solution of $20 \%$ honey and $10 \%$ sucrose) was offered to the butterflies. Inbreeding was minimalized by adding wild butterflies and caterpillars from the Dutch Flevopolder to the existing population. After starving 
for $1 \mathrm{~h}$, first-instar (L1) larvae were placed on Arabidopsis leaves using a fine paint brush as described by Van Wees et al. (2013).

\section{Stress treatments}

Control plants did not receive any stress treatment (Figure 1). For single Pieris treatment, 17-day-old plants were exposed to one Pieris first-instar larva (one larva per one plant)for one day. Thereafter, the caterpillars were removed and the plants were allowed growth under the aforementioned control conditions for six days ("Pieris"). For single drought treatment, 19-day-old plants were irrigated with the nutrient solution (described above) that contained $7.7 \% \mathrm{w} / \mathrm{v}$ polyethylene glycol (PEG) 8000 for six days ("drought"). For combinatorial stress, 17-day-old plants were exposed to one Pieris first-instar larva for one day. The caterpillars were then removed and the plants continued to grow under the control conditions for one day to be subsequently subjected to irrigation with the Hyponex solution containing 7.7 \% w/v PEG 8000 for six days ("Pieris and subsequent drought").

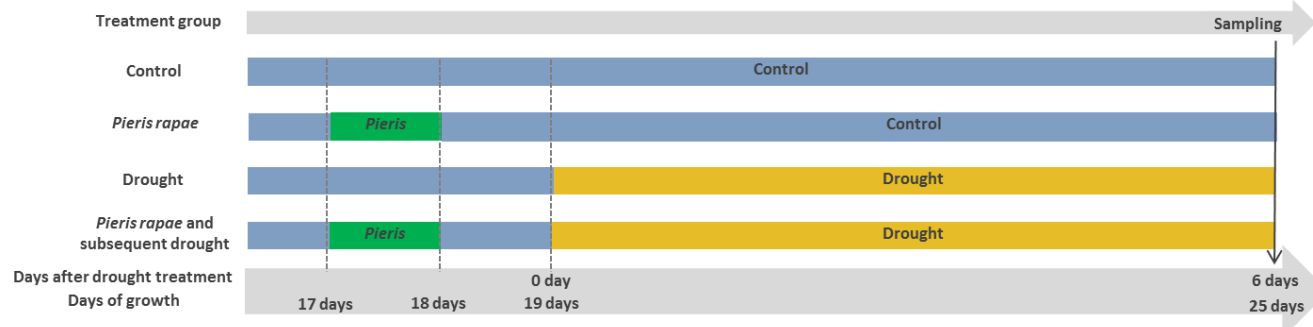

Figure 1. Experimental design of the applied plant treatment. $A$. thaliana plants were subjected to Pieris rapae herbivory, drought, or combinatorial stress (Pieris herbivory and drought) as well as allowed growth under control conditions. The various treatment conditions are indicated in different colors: control, blue; drought, yellow; Pieris, green; rehydration after drought, gray. The vertical arrow indicates the sampling time point. Vertical dashed lines indicate periods before Pieris pre-treatment, after Pieris treatment, and before exposure to drought stress conditions. 


\section{Real-time reverse transcription PCR ( $R R T-P C R$ )}

RNA was extracted from $A$. thaliana rosettes following the protocol proposed by Onate-Sanchez and Vicente-Carbajosa (2008). cDNA was then synthesized from $800 \mathrm{ng}$ of total RNA using the iScript cDNA synthesis kit (Bio-Rad) at $25^{\circ} \mathrm{C}$ for $5 \mathrm{~min}, 42{ }^{\circ} \mathrm{C}$ for $30 \mathrm{~min}$, and $85^{\circ} \mathrm{C}$ for $5 \mathrm{~min}$. CDNA thus obtained was diluted (1:10) and used as a template in quantitative PCR (qPCR). According to the transcriptome data collected from several studies concerning plant responses to drought, osmotic, and Botrytis infection stress triggers, relying on the Genevestigator (http://refgenes.org/rg) web tool application, RETICULONLIKE PROTEIN B11 (RTNLB11) proved the gene characterized by the most stable expression level across the experiments and was, therefore, selected as a reference gene. The qPCR was performed using the $\mathrm{iQ} \mathrm{SYBR}^{\circledR}$ Green Supermix (Bio-Rad) on the CFX96 real-time PCR system (Bio-Rad) set to $95^{\circ} \mathrm{C}$ for $4 \mathrm{~min}$, followed by 40 cycles of $95{ }^{\circ} \mathrm{C}$ for $10 \mathrm{sec}$ and $55{ }^{\circ} \mathrm{C}$ for $30 \mathrm{sec}$. Primer sequences of all investigated genes are provided in Supplementary Table 1.

\section{Statistical analysis}

Broad-sense heritability, $\mathrm{H}^{2}$ was calculated using equation (1):

$$
\mathrm{H}^{2}=\frac{\operatorname{Var}(G)}{\operatorname{Var}(G)+\operatorname{Var}(E)}
$$

where $G$ stands for genotype, $E$ for environment and Var for variance.

Pearson's correlation coefficient ( $r$ ) analysis, one-way, two-way, and threeway analysis of variance (ANOVA, to test the Accession $\times$ Gene $\times$ Treatment interaction), as well as canonical variate analysis (CVA) were performed using the GenStat software ( $16^{\text {th }}$ Edition). The three-way ANOVA used to assess the Accession $\times$ Pieris $\times$ Drought interaction was performed as described by Sokal RR (1981). The logarithm (log) transformed quantification cycle $\left(C_{q}\right)$ values of drought-responsive genes were used for the three-way ANOVA tests. Student's t-test was used to compare the investigated accessions subjected to two alternative treatments. Statistical tests on the measured parameters: rosette fresh weight (FW), rosette dry weight (DW), and rosette water content (WC) 
ratio were performed upon four plants per accession. The water content (WC) ratio was calculated using equation (2):

$$
W C=\frac{F W-D W}{F W}
$$

Statistical tests on the log transformed $\mathrm{C}_{\mathrm{q}}$ values were performed upon three plants per accession. 


\section{Results}

\section{Effect of Pieris rapae herbivory and subsequent drought treatment on 16 natural accessions of Arabidopsis}

Sixteen $A$. thaliana accessions, exhibiting maximum genetic divergence based on SNP marker information (Li et al., 2010), were selected and examined for the effect of Pieris rapae herbivory (Pieris) and/or drought stress on plant performance (Figure 1). Single Pieris treatment significantly reduced the rosette fresh weight (FW) and rosette dry weight (DW) in the accessions Bur-0, Col-0, Got-7, NFA-8, RRS-7, and Tamm2 (Figure 2 a and b). Strikingly, the representatives of Est-1 accession showed significantly higher FW and DW values upon Pieris treatment than under control conditions. Sole drought treatment significantly reduced both FW and DW quotients in Bur-0, Col-0, Fei-0, NFA-8, RRS-10, RRS-7, Tamm-2, and Van-0. Decrease in DW was also detected in Got-7, Sha, and Ts-1, indicating that most accessions experienced serious growth retardation upon drought treatment. This finding prompted us to examine the water content (WC) ratio, which was significantly enhanced in Got7, Sha, Tamm-2, and Ts-1 and decreased in Tsu-0, pointing to the latter as the most drought-susceptible accession (Figure $2 \mathrm{c}$ ). Pieris pre-treatment followed by that simulating drought resulted in lowered FW values in Bur-0, Col-0, Est-1, Got-7, RRS-7, and Tamm2 and diminished DW rates in Est-1 and RRS-7, in comparison to those characteristic of plants subjected to drought conditions without pre-treatment. Significantly reduced WC values were observed in Bur-0, Col-0, Got-7, Lov-5, Sha, Tamm-2, and Ts-1, showing that the most droughtresistant accessions (evaluated as such based on their WC ratio parameters) lost their relative drought tolerance after Pieris pre-treatment. Broad-sense heritabilities of the investigated traits were high, mostly above 0.5 , up to 0.65 , with the exception of the shift in WC ratio upon Pieris treatment, which showed moderate heritability (Table 1 ). Results obtained thus far offered a favorable prospect for genetic analysis of the test traits in the 16 accessions. 

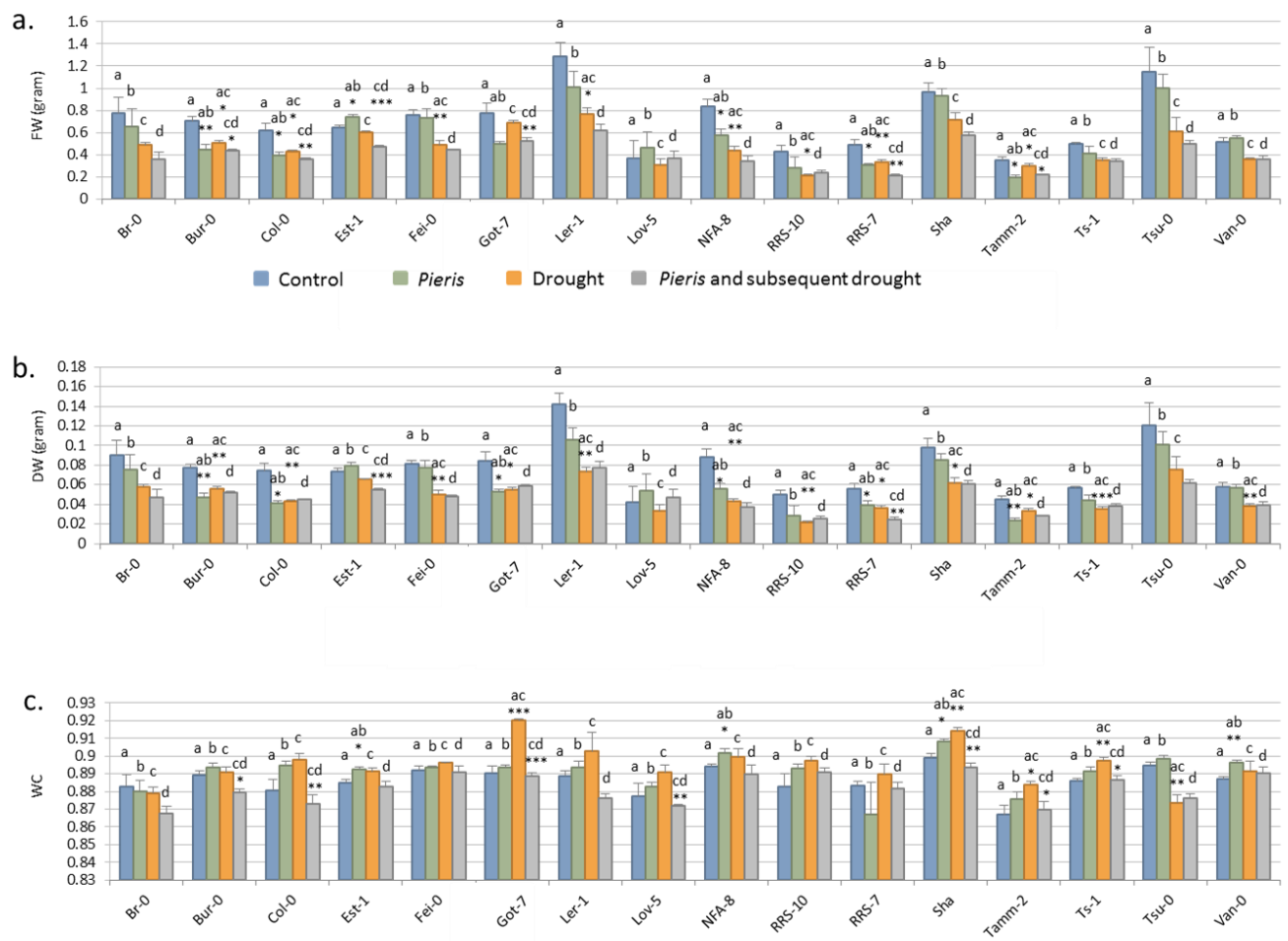

Figure 2. Phenotypic analysis of rosettes of $16 \mathrm{~A}$. thaliana accessions in response to Pieris rapae herbivory, drought, and drought induced after Pieris pre-treatment. a., Rosette fresh weight (FW); b., rosette dry weight (DW); c., water content (WC), calculated as the (FW-DW)/FW ratio. Phenotypes observed under control conditions are indicated in blue, those resulting from exposure to Pieris alone - in green, drought alone - in yellow, and Pieris pre-treatment followed by drought - in grey. The standard error of the mean (SE) was calculated based on four replicates. Two-way ANOVA was used to compare Pieris- and drought-induced responses to the control and to compare combinatorial stress feedback to that caused by drought. $p$-values: $*<0.05, * *<0.01, * * *<0.001$

Table 1. Broad-sense heritability, $\mathrm{H}^{2}$ of rosette fresh weight (FW), dry weight (DW), and water content (WC $=$ FW-DW/FW) of $16 \mathrm{~A}$. thaliana accessions upon exposure to Pieris rapae herbivory, drought, and combinatorial stress conditions $(n=4)$.

\begin{tabular}{c|c|c|c}
\hline Treatment & FW & DW & WC \\
\hline Control & 0.63 & 0.60 & 0.59 \\
Pieris & 0.58 & 0.55 & 0.37 \\
Drought & 0.65 & 0.60 & 0.64 \\
Pieris and drought & 0.63 & 0.59 & 0.53 \\
\hline
\end{tabular}




\section{Correlations among traits}

Pearson's correlation coefficients between FW, DW, and WC were calculated for each set of experimental conditions. Positive correlations between FW and DW were found in all investigated settings. While the correlation between FW and WC was significant under all applied conditions except the combinatorial treatment, the latter correlation was largely lost when considering DW and WC upon sole drought induction (Table 2). This observation pointed to WC as an informative trait in the study of sensitivity to drought.

Table 2. Pearson's correlation coefficients upon comparison of rosette fresh weight (FW), dry weight (DW), and water content (WC) of $16 \mathrm{~A}$. thaliana accessions under each set of experimental conditions. Significant correlations are shaded in gray.

\begin{tabular}{c|c|c|c}
\hline & \multicolumn{3}{|c}{ Phenotypic traits } \\
\hline \multirow{2}{*}{ Treatment group } & & DW & WC \\
\hline \multirow{2}{*}{ Pontrol } & FW & 0.99 & 0.61 \\
\cline { 2 - 4 } & WC & 0.47 & \\
\hline \multirow{2}{*}{ Drought } & FW & 0.98 & 0.49 \\
\cline { 2 - 4 } & WC & 0.32 & \\
\hline \multirow{2}{*}{ Pieris and subsequent drought } & FW & 0.94 & 0.32 \\
\cline { 2 - 4 } & WC & -0.01 & \\
\cline { 2 - 4 } & FW & 0.97 & 0.17 \\
\hline
\end{tabular}

To observe the variation of phenotypic responses of the investigated accessions under stress conditions, three-way ANOVA was performed to test the interactions between traits, treatments, and accessions. Significant main effects of accessions, traits, and treatments were observed ( $p<0.001$; Table 3$)$ and significant interactions between accessions, traits, and treatments were found. Upon exposure to divergent treatments, the plants showed a large variation in phenotypic responses. 
Table 3. Three-way ANOVA to test the interaction between accessions, traits, and treatments. All main effects and interactions were significant.

\begin{tabular}{|c|c|c|c|c|c|}
\hline Source of variation & d.f & S.S & $\mathbf{m . s}$ & $\mathbf{v . r}$ & $p$-value \\
\hline Accessions (Acc) & 15 & 7.98 & 0.53 & 69.91 & $3.06 \mathrm{E}-132$ \\
\hline Traits & 2 & 211.82 & 105.91 & 13914.40 & $0.00 \mathrm{E}+00$ \\
\hline Treatments & 3 & 2.32 & 0.77 & 101.71 & $1.96 \mathrm{E}-55$ \\
\hline Acc $\times$ Traits & 30 & 3.96 & 0.13 & 17.35 & $3.96 \mathrm{E}-67$ \\
\hline Acc $\times$ Treatments & 45 & 1.18 & 0.03 & 3.45 & $1.75 \mathrm{E}-12$ \\
\hline Traits $\times$ Treatments & 6 & 1.21 & 0.20 & 26.46 & $1.04 \mathrm{E}-28$ \\
\hline Acc $\times$ Traits $\times$ Treatments & 90 & 0.67 & 0.01 & 0.98 & $5.34 \mathrm{E}-01$ \\
\hline Residual & 576 & 4.38 & 0.01 & & \\
\hline Total & 767 & 233.53 & & & \\
\hline
\end{tabular}

To test the interactions between treatments and accessions, three-way ANOVAs were performed for the three traits separately (Table 4 ). The analyses showed significant main effects of Pieris and drought treatments $(p<0.001)$ in all cases, as well as interaction effects between accessions and the two single stress triggers (Pieris and drought) for FW and DW $(p<0.001)$ and between accessions and drought-simulating treatment regarding WC $(p<0.001)$. The only significant interaction effect between accessions, Pieris and drought was observed for WC $(p<0.05)$.

Taken together, the obtained results point to a large variation in the phenotypic response of plants exposed to Pieris herbivory, drought, or the combination of stress triggers (exposure to Pieris followed by drought simulation). Calculation of the WC ratio allows identification of natural variation in the drought response, including the influence of Pieris pre-treatment. 

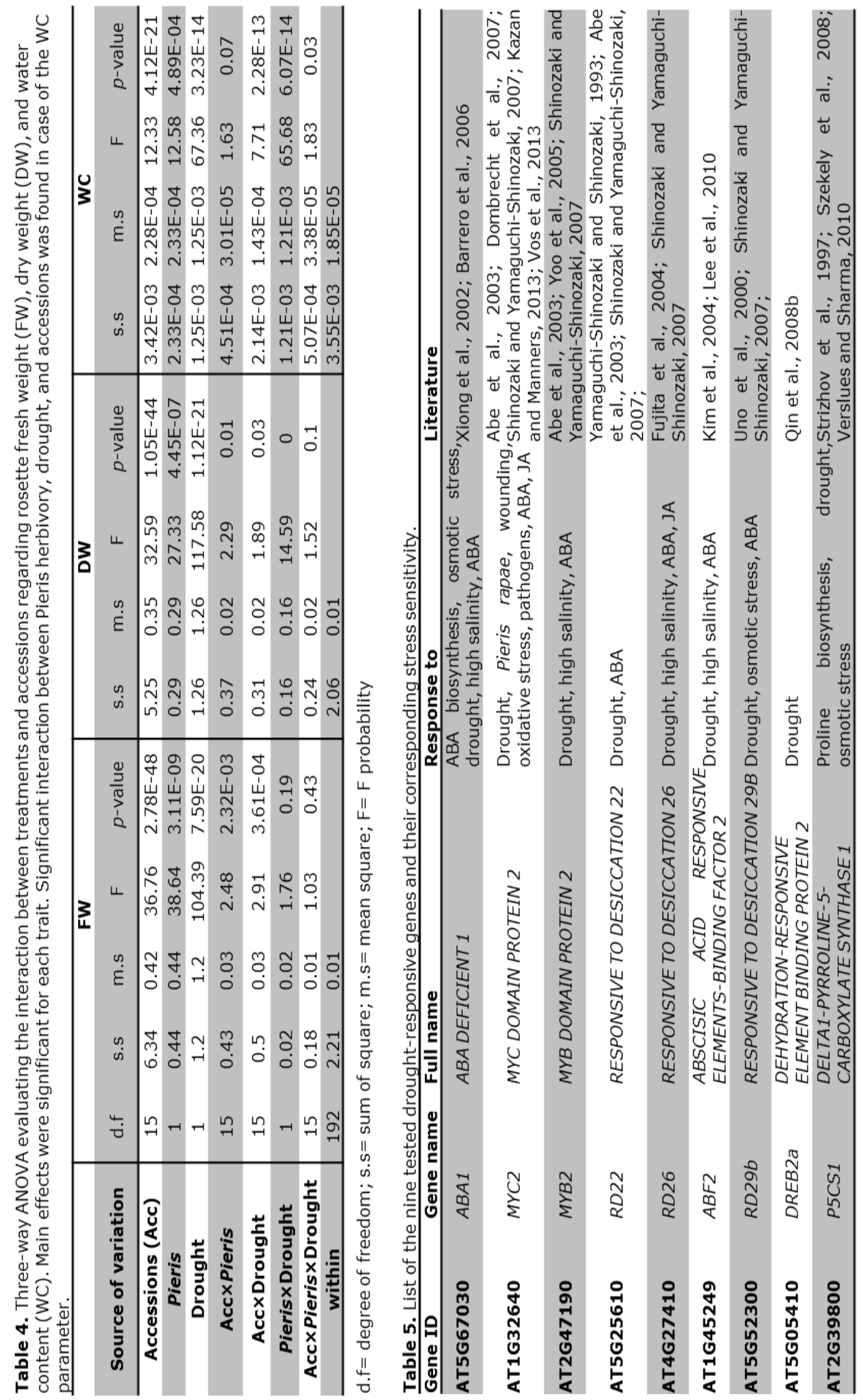


\section{Gene expression variation under Pieris, drought, and the combinatorial treatments}

To explore whether the genetic background and the observed phenotypic differences influenced the transcriptional output of the investigated plant population upon Pieris, drought, and the combinatorial stress treatments, expression levels of nine known drought-responsive genes (Table 5) were compared to those characteristic of plants maintained under control conditions. The expression quotients of interest were determined via analysis of the genetic material of rosette leaves of 14 Arabidopsis accessions, as the relevant organs of the other two accessions were lost during harvesting. All primers used for the quantitative reverse transcription PCR ( $q R T-P C R$ ) analysis were designed to amplify the same coding sequence region from the mRNA pools of the investigated accessions and were pre-tested for efficiency.

Pearson's correlation coefficients between the three investigated traits and the nine drought-responsive gene expression levels were calculated and significant correlations were found (Supplementary Table 4), while the obtained transcription quotients could not be correlated with the treatment effects on growth of the tested natural accessions of Arabidopsis.

When examining gene expression responses in general, two groups of accessions could be distinguished; one, for which ${ }^{2} \log$ quantification cycle $\left(\mathrm{C}_{\mathrm{q}}\right)$ values $\left({ }^{2} \log C_{q}\right)$ decreased upon drought treatment but increased upon application of the combination of Pieris and sequential drought triggers and another, showing hardly any response to drought but a decrease in ${ }^{2} \log C_{q}$ values upon exposure to the combinatorial stress treatment (Figure 3). Further, divergent effects of the Pieris pre-treatment in response to sequential drought were found in the investigated accessions. 


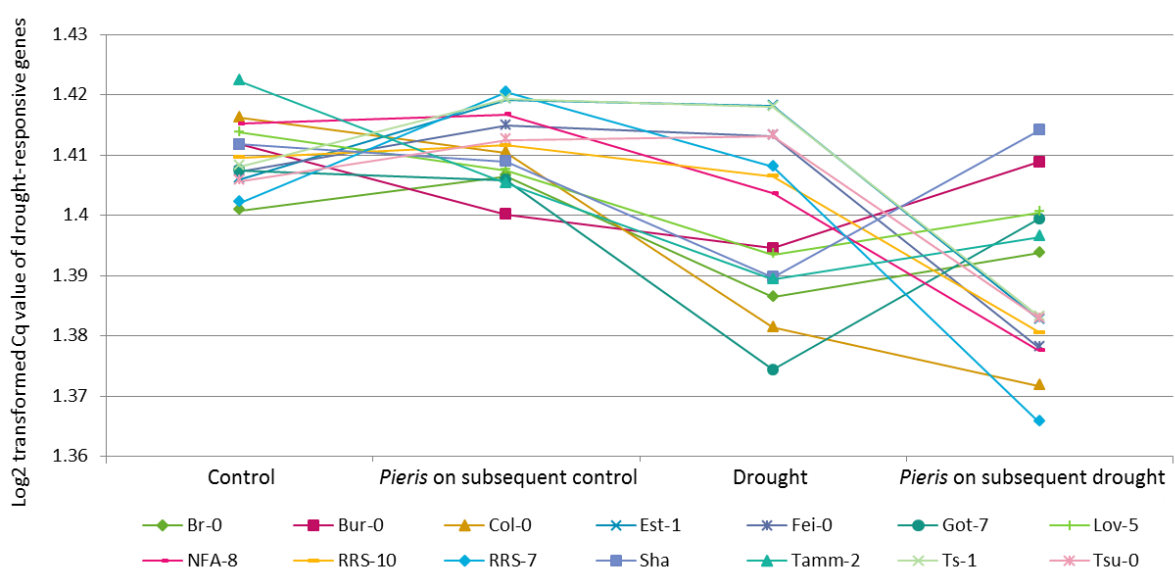

Figure 3. Variation in mean average $C_{q}$ values (on a log2 scale) representing expression of the nine investigated drought-responsive genes in rosettes of $14 \mathrm{~A}$. thaliana accessions in response to drought, Pieris herbivory, and Pieris pre-treatment followed by drought simulation.

Pearson's correlation coefficients between gene expression levels were calculated using relevant $\mathrm{C}_{\mathrm{q}}$ values. For most genes, a positive correlation was observed (Table 6), while a negative one was noted between $A B A 1$ and DREB2a quotients, confirming their independence. No significant correlation was found between MYB2 transcript abundance values and those of the remaining investigated genes, showing that MYB2 is not related to other droughtresponsive genes in the three tested experimental settings. 
Table 6. Pearson's correlation coefficients of the investigated drought-responsive genes calculated on the basis of the relevant $C_{q}$ values. Gray shading indicates significant correlation $(p<0.05)$.

\begin{tabular}{|c|c|c|c|c|c|c|c|c|c|}
\hline$A B A 1$ & 1 & & & & & & & & \\
\hline$A B F 2$ & 0.17 & 1 & & & & & & & \\
\hline$D R E B 2 a$ & -0.31 & 0.26 & 1 & & & & & & \\
\hline MYB2 & 0.11 & -0.03 & 0.02 & 1 & & & & & \\
\hline MYC2 & -0.02 & 0.47 & 0.4 & -0.06 & 1 & & & & \\
\hline P5CS1 & 0.36 & 0.62 & -0.04 & -0.09 & 0.61 & 1 & & & \\
\hline$R D 22$ & -0.11 & 0.51 & 0.57 & 0.09 & 0.76 & 0.48 & 1 & & \\
\hline$R D 26$ & 0.08 & 0.45 & 0.42 & -0.08 & 0.72 & 0.62 & 0.61 & 1 & \\
\hline$R D 29 b$ & 0.20 & 0.70 & 0.18 & 0.02 & 0.32 & 0.54 & 0.31 & 0.4 & 1 \\
\hline Genes & $A B A 1$ & $A B F 2$ & DREB2a & MYB2 & MYC2 & P5CS1 & $R D 22$ & $R D 26$ & $R D 29 b$ \\
\hline
\end{tabular}

To assess the effect of the applied treatments on plant gene expression, canonical variate analysis (CVA) was performed upon the relevant $\mathrm{C}_{\mathrm{q}}$ values. CVA is a multivariate analysis method that has a similar function to the principle component analysis (PCA) applied to evaluate the possible correlation between variables (Heymann and Noble, 1989). The difference is that CVA can be used to discriminate between alternative treatment groups as it accounts for differences in group variation in course of their separation (Heymann and Noble, 1989). Thus, the CVA axes maximize the variance between groups relative to that within them (Zelditch et al., 2012). The resulting biplot (Figure 4) represents $92.9 \%$ of total variance and shows that the variation among accessions was lower than the variation among treatments, based on the separation of plants subjected to control conditions, drought, and the combination of Pieris and subsequent drought simulation, but not on separation of accessions. As a result, strong effects of Arabidopsis accessions on gene expression come to the fore, while those of single stress treatments cannot be distinguished in the presented plot. When considering individual components, the CVA-1 component explained $77.6 \%$ of the total variance, especially for DREB2a, RD22, P5CS1, and RD29b while the CVA-2 explained $15.3 \%$ of the total variance, most notably in case of ABF2, RD22, MYC2, P5CS1, and DREB2a (Supplementary Table 2). 


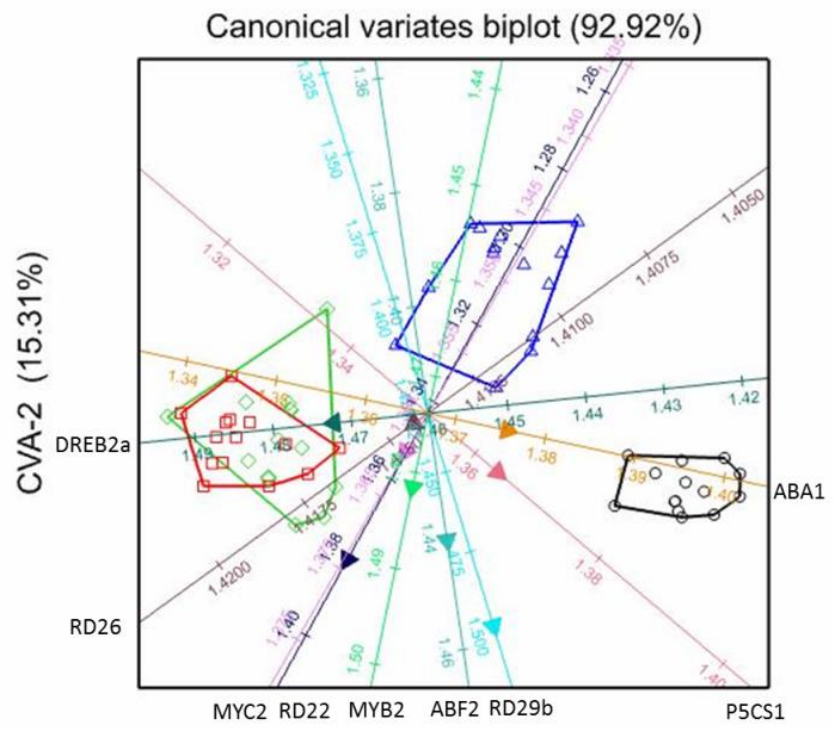

CVA-1 $(77.61 \%)$

\begin{tabular}{|ll|}
\hline$\circ$ & Control \\
$\square$ & Drought \\
& Pieris \\
$\triangle$ & Pieris and subsequent drought \\
\hline
\end{tabular}

Figure 4. Canonical variates analysis (CVA) explained $92.92 \%$ of total variance in drought-responsive gene expression upon application of alternative stress treatments. The CVA-1 explained $77.61 \%$ of total variance and the CVA-2, $15.31 \%$. The biplot displays a clear separation between the control group, the one subjected to drought, and that exposed to combinatorial stress. Each biplot axis represents one gene, while the numbers on the axes, the corresponding $C_{q}$ values on the log 2 scale. The angles between the axes illustrate correlation between the variables and arrows, the direction, with lines in opposite direction indicating negative correlation (e.g., DREB2a and ABA1). Black color represents control conditions; green, Pieris treatment; red, drought simulation; blue, combinatorial stress induction. Each dot within each treatment group represents one accession.

As initially expected, drought stress (as compared to control conditions) induced the expression of ABA-dependent signaling genes $A B A 1, R D 26, M Y C 2$, $R D 22, R D 29 b, A B F 2$, and P5CS1, although the levels of induction were quite variable between accessions (Figure 5). Strikingly, the ABA-dependent signaling gene MYB2 was either up- or down-regulated depending on the accession. For instance, its expression fold change under drought conditions (relative to control) was two times lower in Fei-0 $(\log 2 \mathrm{FC}=-1)$ but up to four times higher in Ts-1 (log2FC= 2). Also DREB2a, an ABA-independent signaling gene showed variable response levels upon comparison of accessions. 
Unexpectedly, analysis of the single Pieris treatment outcomes also revealed differential expression of drought-responsive genes depending on their genetic background (Supplementary Figure 1). RD29b and P5CS1 were up-regulated, whereas the expression levels of $R D 26, R D 22$, and $D R E B 2 a$ decreased. This observation suggested that transcriptional activity of the aforementioned genes, thought to be largely drought-responsive, was also susceptible to biotic stress triggers (such as Pieris herbivory), thus leading us to hypothesize that, depending on the genetic background, a combination of stress factors can have a synergetic or an antagonistic effect on the expression levels of droughtresponsive genes. To address this hypothesis, gene expression levels were analyzed by comparing the drought vs. control expression difference with the combinatorial stress vs. control expression difference (Figure 5). Pieris pretreatment reduced the expression of $A B A 1$ and P5CS1 in response to sequential drought, when compared to the outcomes of the single drought treatment, while MYB2, RD22, and ABF2 were up-regulated. For RD26, MYC2, and DREB2a, the direction of the response varied considerably depending on the accession, indicating a large effect of natural genetic variation on their transcriptional activity. This supposition was confirmed by a three-way ANOVA, accounting for $C_{q}$ values of drought-responsive genes, that showed a significant main effect of drought stress for all genes ( $p<0.001$, Supplementary Table 3$)$ and a significant main effect of Pieris on the expression levels of RD26, MYB2 DREB2a, RD29b, and $A B F 2(p<0.05)$. Moreover, significant interactions were found between genes and accessions, meaning that a combination of biotic and abiotic stress elicits a specific response in each accession. 
a.

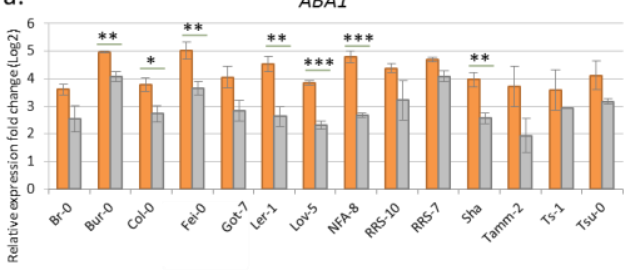

घrought $\quad$ Pieris and subsequent drought
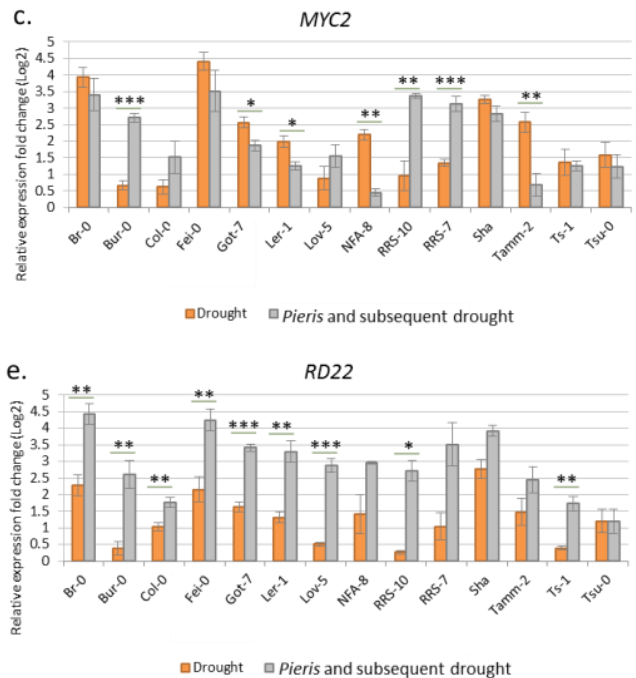

Drought $\square$ Pieris and subsequent drought
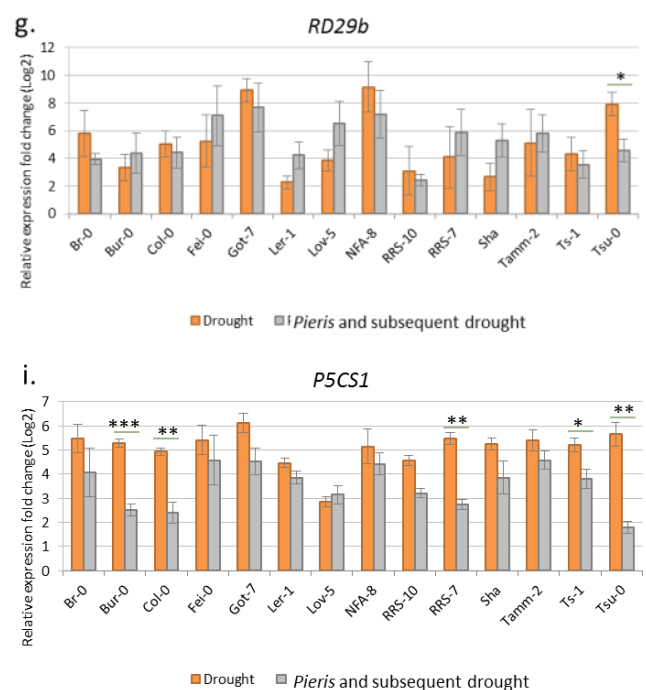

b.

$R D 26$

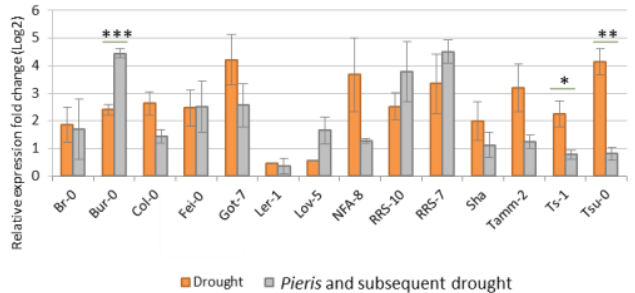

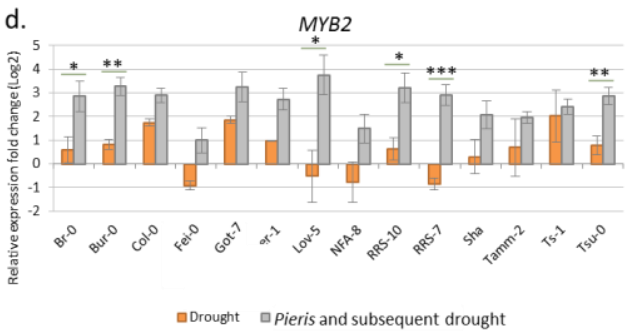

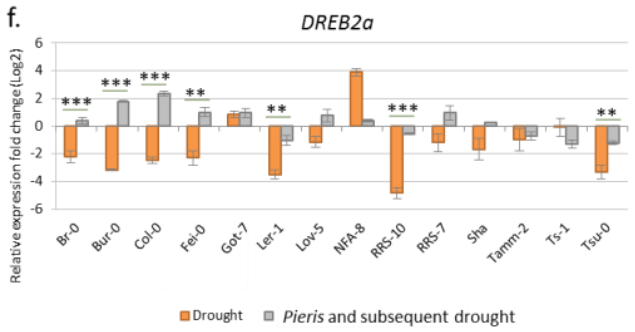

h.

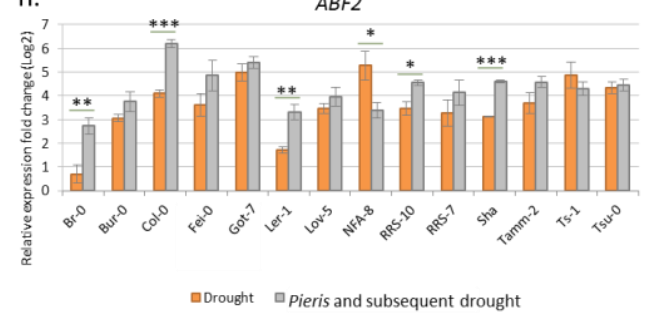


Figure 5. Relative expression levels of drought-responsive genes: a., $A B A 1$; b., RD26; C., MYC2; d., MYB2; e., RD22; f., DREB2a; g., RD29b; h., AFB2; i., P5CS1 in rosettes of the investigated $A$. thaliana accessions upon comparison of drought vs. control and combinatorial stress vs. control treatments. Log2 scale at $1,2,3,4,5,6,7$, and 8 , corresponds to $2,4,6,8,16,32,64,128$, and 256 times actual fold change, respectively. The standard error of the mean (SE) was calculated based on three plants per accession. Two-way ANOVA was used to compare the responses to the combinatorial stress to those caused by drought simulation. Significant differences ( $p$-values): $*<0.05, * *<0.01, * * *$ $<0.001$. 


\section{Discussion}

This work uncovers the differences in responses of natural accessions of $A$. thaliana to single and combinatorial stress treatments involving biotic stress imposed by herbivory of $P$. rapae larvae and abiotic stress due to drought.

\section{WC is a suitable parameters to present drought response}

Our research showed that the water content ratio (WC, (FW-DW)/FW), but not the fresh weight (FW) or the dry weight (DW) parameters of Arabidosis rosettes alone, is a trait relevant to identification of genotypes that are either tolerant, susceptible, or characterized by mechanisms aiming at evasion of detriments of drought. Low WC ratio values coincide with inhibited plant growth, as the parameter is positively correlated with solutes and metabolic activity in plants (Bac-Molenaar et al., 2016; Loudet et al., 2003).

\section{Different responses to drought stress in Arabidopsis}

Drought tolerance is characterized by maintaining similar DW and WC quotients under drought stress to those corresponding to control conditions, which was observed in Br-0, Est-1, and Lov-5. Further, Tsu-0 exhibited drought tolerance, which is consistent with earlier findings on the relative water content in this accession under drought conditions (Bouchabke et al., 2008). In contrast, accessions Bur-0, Col-0, Fei-0, Ler-1, NFA-8, RRS-10, RRS-7, and Van-0 proved drought-sensitive, displaying reduced DW values but no differences in WC.

Under drought stress, plants can avoid dehydration by retaining water, reducing photosynthesis, and enhancing root development (Verslues et al., 2006). Drought avoidance is characterized by diminished DW outputs along with increased WC. Such phenotypes were found for the accessions Got-7, Sha, Tamm-2, and Ts-1. Several potential downstream targets of the two floweringtime regulators, FRIGIDA (FRI) and FLOWERING LOCUS C (FLC), were identified by QTL mapping of WC under two contrasting nitrate regimes (Loudet et al., 2003). FRI can activate the expression of FLC, which inhibits flowering by repressing transcription of downstream genes in the floral pathway (Michaels and Amasino, 1999). It is, moreover, possible that FLC is involved in WC regulation through quenching of the downstream flowering integrator 
SUPPRESSOR OF OVEREXPRESSION OF CO 1 (SOC1) to control stomatal opening (Kimura et al., 2015; Loudet et al., 2003). These considerations suggest that Got-7, Sha, Tamm-2, and Ts-1 (early flowering accessions) mobilize drought-avoidance mechanisms by accelerating their metabolic processes to finish their life cycle with limited biomass accumulation.

Another way to circumvent sensitivity to drought is for plants to stay small, as their high water use efficiency (WUE) renders smaller specimens more tolerant to the stress factor (Blum, 2005). In the present study, five accessions characterized by low biomass were investigated, Lov-5, RRS-10, RRS-7, Tamm2, and Ts-1; of these, however, only Lov-5 proved drought-tolerant. Our observation of different responses of Arabidopsis accessions to drought stress indicates a variation in plant adaptation to extreme environments.

\section{Role of drought-responsive genes in plant stress mechanisms triggered by consecutive Pieris and drought treatments}

In our study, expression of all investigated genes (except RD29b) was affected by the interaction between plant response mechanisms triggered by Pieris herbivory and those caused by the subsequent drought stress, with both the ABA-dependent and the ABA-independent signaling pathways involved. The cross-talk between the aforementioned stress response cascades was described by Nakashima et al. (2014).

$A B A 1$ is involved in $A B A$ biosynthesis and is down-regulated by $\mathrm{JA}$ (Anderson et al., 2004; Xiong and Zhu, 2003). Enhanced $A B A 1$ expression under drought simulating conditions observed in this study points to increased accumulation of the phytohormone in response to the stress factor (Figure 5 a). In contrast, reduced transcriptional activity of $A B A 1$ detected under combinatorial stress conditions indicates a negative interaction between the Pieris-induced JA signaling pathway and the drought-activated ABA-dependent response mechanism. The aforementioned results proved our experimental design sufficient for the study of the interaction between Pieris- and drought-triggered stress responses in planta. 
MYB2 and MYC2 are transcription factors acting as positive regulators of Pieris and drought stress responses, thus playing an important role in the crosstalk between ABA and JA signaling trails (Abe et al., 2003; Anderson et al., 2004; Harb et al., 2010). Herein, Pieris pre-treatment enhanced MYB2 expression in plants subsequently exposed to drought. This finding is in accord with previously reported results showing that $M Y B 2$ can be respectively up- and down-regulated by two upstream transcription factors, WRKY18 and WRKY40 (Shang et al., 2010), positively responding to JA (Xu et al., 2006). Further, opposite effects of Pieris pre-treatment on MYC2 gene expression were observed between the investigated accessions. Both MYB2 and MYC2 bind to the promoter region of $R D 22$ activating its transcription (Abe et al., 2003). In our study, exposure to Pieris larvae resulted in RD22 up-regulation in most accessions upon subsequent drought simulation, when compared to the single drought treatment. This finding exemplifies a synergetic effect of Pieris pre-treatment and drought simulation, as $R D 22$ was repressed in plants exposed to the herbivore alone.

Enhanced expression of DREB2a under a 24-hour dehydration treatment (relative to control) was reported previously (Yamaguchi-Shinozaki and Shinozaki, 1994). Herein, however, the gene was down-regulated in most accessions after six days of drought simulation. As the increased severity of drought stress is positively associated with the concentration of ABA in plants (Kim et al., 2012), the observed reduction in DREB2a transcriptional activity could be due to its sensitivity to the phytohormone levels. To confirm this supposition, measurements of ABA concentration in plants on consecutive days of drought treatment are necessary. Moreover, a positive influence of Pieris herbivory on DREB2a expression under subsequent drought conditions was observed, when compared to the single drought treatment. The effect could be due to the interaction between salicylic acid (SA), JA, and ABA activity in Arabidopsis plants. According to Yoshioka and Shinozaki (2009), the involvement of $D R E B 2 a$ in the cross-talk between plant hormones is relevant to the interaction of SA and ABA rather than that between ABA and JA. Thus, it is possible that the effect of Pieris pre-treatment on DREB2a gene expression is not JA- but SA-dependent. 
Expression of $R D 29 b$ is regulated by several transcription factors, encoded by drought-responsive genes, such as ABF2 (Uno et al., 2000), DREB2a (Qin et al., 2008a), and ANAC019 (that can be further modulated by MYB2 and MYC2 (Bu et al., 2008; Hickman et al., 2013; Jensen et al., 2010). The former TF can

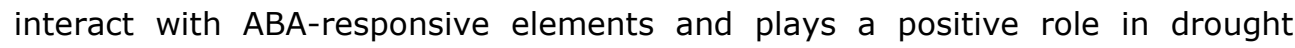
feedback (Abe et al., 2003). In this study, we expected Pieris pre-treatment to lead to up-regulation of ANACO19 and, thus, exert an effect on RD29b expression under sequential drought stress conditions. However, no significant differences in $R D 29 b$ transcriptional activity under combinatorial stress (relative to single drought treatment) were observed between the investigated accessions (except Tsu-0). This could be due to the complex regulation of $R D 29 b$ expression in response to drought. For instance, besides ANAC019, ABF2, and DREB2a, positive regulators of $R D 29 b$ whose gene expression increases under drought conditions, transcription of the gene in question can be further increased by ETHYLENE INSENSITIVE 2 (EIN2) and SA- AND ABADOWNREGULATED ZINC FINGER GENE (SAZ) whose levels decrease under drought treatment (Jiang et al., 2007; Wang et al., 2007).

Furthermore, enhanced expression of P5CS1, pointing to increased proline biosynthesis, was observed in all investigated accessions upon drought simulating treatment. The promoter region of P5CS1 contains a droughtresponsive element (DRE) which can be bound by the JA-responsive protein ETHYLENE RESPONSE FACTOR 1 (ERF1) (Anderson et al., 2004), with the latter negatively regulated by MYC2, VSP2, and ABA treatment (Anderson et al., 2004; Dombrecht et al., 2007). The factor can boost P5CS1 expression, as was found in 35S:ERF1 plants (Cheng et al., 2013). Thus, the subsequently observed reduced expression of P5CS1 under combinatorial stress conditions could be due to the antagonistic interaction between the down-regulation of ERF1 and increased proline accumulation characteristic of drought exposure.

Based on the one described by Shinozaki and Yamaguchi-Shinozaki (2007), we hereby propose a model of gene regulatory networks relevant to the consecutive Pieris and drought stress responses in plants (Figure 6). Our study focused on measurement and assessment of the effect of Pieris herbivory on expression levels of known drought-responsive genes upon sequential drought 
exposure; we, however, payed less attention to Pieris-responsive genes. Some of the latter, such as ERF1, ANAC019, and VSP2, can be regulated by both JA and ABA. Thus, it would be of further interest to determine the effect of Pieris pre-treatment on transcriptional activity of the three genes in response to sequential drought simulation. No effect of the interaction between Pieris and drought stress triggers was observed in case of $R D 29 b$ only. Our hypothesis as to the cause of this outcome pertains to the aforementioned complexity of its upstream regulation. For instance, since EIN2 and SAZ were identified as involved in down-regulation of $R D 29 b$ in response to drought stress, additional evaluation of expression levels of their encoding genes is necessary. Lastly, to comprehensively delineate the gene regulatory network triggered in response to the combinational stress in planta, full transcriptome analysis is called for.

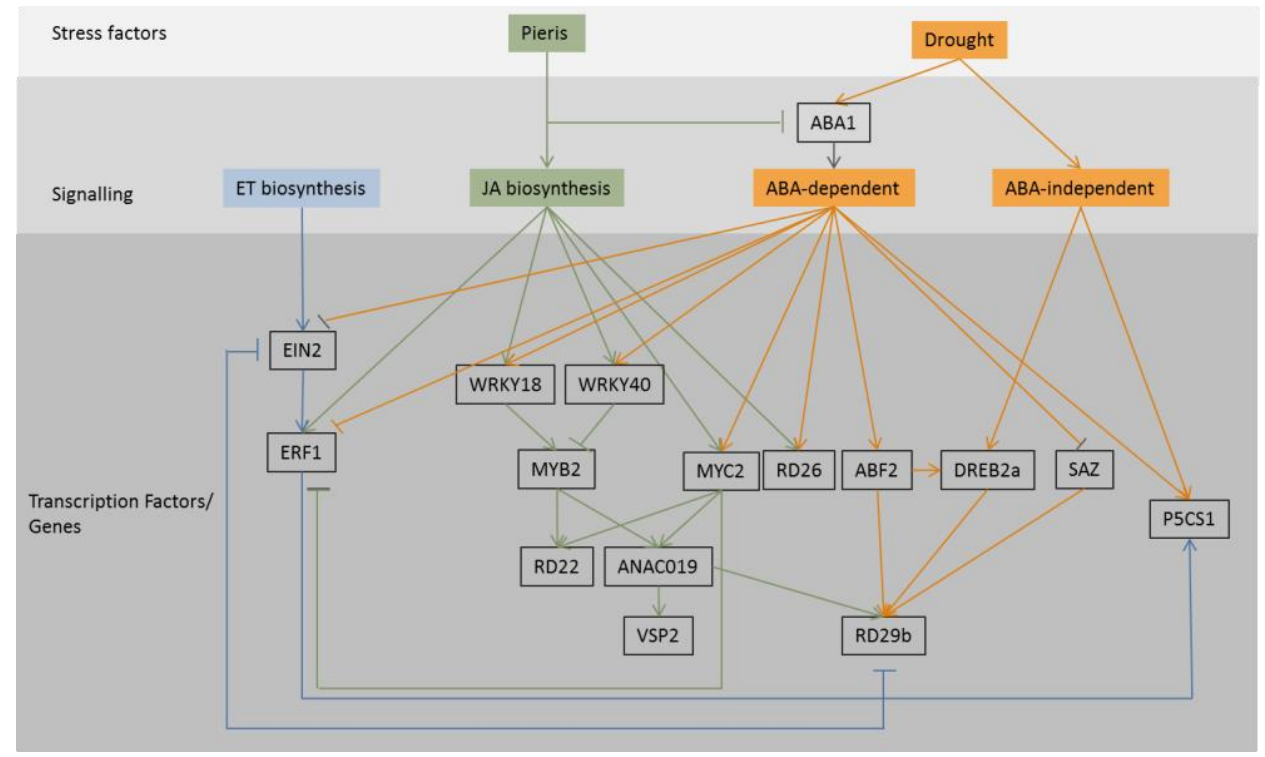

Figure 6. Model of the gene regulatory networks relevant to the consecutive Pieris and drought stress responses in plants, involving $A B A 1, M Y B 2, M Y C 2, R D 22, R D 26, A B F 2$, DREB2a, RD29B, P5CS1, WRKY18, WRKY 40, ANAC019, VSP2, EIN2, and ERF1. Signaling pathways triggered by drought are indicated in orange, while those implicated in the response mechanism to Pieris rapae herbivory are marked in green. The figure was updated based on Shinozaki and Yamaguchi-Shinozaki (2007). 


\section{No divergent expression profiles of the investigated drought- responsive genes among Arabidopsis accessions}

Our findings on differential gene expression are in line with previous results of a study comparing seven Arabidopsis accessions in response to SA treatment that identified a number of genes with multiple alleles characterized by diversified transcriptional activity (Kliebenstein et al., 2006). Similarly, contrasting expression of abiotic stress-responsive genes (including RD22) was observed in Col-0, when compared with C24 (Miller et al., 2015). Moreover, diverse expression levels in response to drought and cold stress were detected upon comparative analysis of ten Arabidopsis accessions (including Bur-0, Col-0, and Ts-1; Lasky et al., 2014). Further, while similar expression of REDUCED CHLOROPLAST COVERAGE 3 (REC3) was reported for the three aforementioned accessions subjected to drought (relative to control), ETHYLENE RESPONSE FACTOR022 (ERF022) showed contrasting transcriptional levels in Ts-1 as compared to Bur-0 and Col-0 (Lasky et al., 2014). We, however, did not observe divergent expression profiles of the investigated drought-responsive genes in Ts1 relative to Bur-0 and/or Col-0, except in case of RD26.

\section{No correlation between phenotypic responses and variation in gene expression}

In the present study, no correlation between the three investigated traits and the nine drought-responsive genes was found (Supplementary Table 1). In addition, the relevant expression level quotients could not be correlated with the treatment effects on growth in Arabidopsis natural accessions. Hence, the obtained results suggest that the representatives of the investigated accessions employ not one, but different strategies to overcome drought stress, or at least not one involving the genes whose expression was evaluated. Considering the substantial variation in both phenotypes and gene expression levels among accessions upon comparison of their responses to single and combinatorial stress treatments, their whole-transcriptome analysis would be of great interest. Since we selected accessions that are genetically divergent, the observed large variation among them was not unexpected. 
Thus, the limited transcriptomic information at our disposal prevented us from effective determination of the relationship between phenotype variation and genetic responses among accessions. For instance, correlation analysis of the expression data of more than 400 Pseudomonas syringae $p v$ tomato DC3000 (Pst) type III effector protein AvrRpt2-responsive genes showed that $49 \%$ of variation of the phenotypic responses to Pst infection could be explained by genetic differences among accessions (Van Poecke et al., 2007). Therefore, full transcriptome analysis of the accessions of interest would be useful in providing adequate genetic information corresponding to stress conditions (relative to control). Another hypothesis aiming at explanation of the poor correlation is that the simple Pearson's test is not sufficient to determine the relationship between complicated phenotype stress responses and relevant genotype data. Application of powerful analytical methods, such as algorithm locally linear embedding graph generator (LEGG) (Van Poecke et al., 2007) and cluster analysis, could help to effectively tackle these obstacles. 


\section{Conclusion}

In this paper, we have evaluated phenotypic responses and gene expression in Arabidopsis upon exposure to Pieris herbivory, drought, and combinatorial stress conditions. The observed responses of the investigated accessions to drought simulation were divergent, indicating a variation in plant adaptation to extreme environments. We identified the phenotypic trait of water content (WC) as a parameter allowing effective estimation of the effects of the interaction between Pieris and drought stress responses. No significant correlation between phenotypic responses and gene expression was found, suggesting that 1) whole transcriptomes of the investigated accessions ascertaining sufficient genetic information and 2) application of superior correlation analysis methods, such as LEGG, will be needed to delineate the relevant interactions. Different effects of Pieris pre-treatment on drought-responsive gene expression levels upon sequential drought simulation were observed, with the exception of $R D 29 b$. Based on the obtained results, we proposed an improved model of gene regulatory networks triggered in response to consecutive Pieris and drought stress factors. However, to afford a comprehensive overview of the network in question, appropriate full transcriptome analyses are necessary. 


\section{Acknowledgement}

We are grateful to Taede Stoker, Gerrit Stunnenberg, and Pauline Sanderson (Unifarm, Wageningen University, NL) for the help with plant propagation; and the Entomology group of Wageningen University for providing us with Pieris rapae larvae. We thank Prof. Fred van Eeuwijk for the guidance of 3-way ANOVA test in software GenStat for Windows; Dr. Henk Schat for the guidance of with 3-way ANOVA tests for the interaction of Pieris and subsequent drought stresses (VU university Amsterdam, NL); We thank Prof. Maarten Koornneef and Dr. Robert Akkers for critically reading the manuscript and providing useful suggestions. This work was financially supported by The Netherlands Organization for Scientific Research (NWO) through the Technology Foundation, Perspective Programme 'Learning from Nature' [STW10988]. 


\section{Reference}

Abe, H., Urao, T., Ito, T., Seki, M., Shinozaki, K. and Yamaguchi-Shinozaki, K. (2003). Arabidopsis AtMYC2 (bHLH) and AtMYB2 (MYB) Function as Transcriptional Activators in Abscisic Acid Signaling. Plant Cell 15, 63-78.

Anderson, J. P., Badruzsaufari, E., Schenk, P. M., Manners, J. M., Desmond, O. J., Ehlert, C., Maclean, D. J., Ebert, P. R. and Kazan, K. (2004). Antagonistic interaction between abscisic acid and jasmonate-ethylene signaling pathways modulates defense gene expression and disease resistance in Arabidopsis. Plant Cell 16, 3460-79.

Atkinson, N. J. and Urwin, P. E. (2012). The interaction of plant biotic and abiotic stresses: from genes to the field. J Exp Bot 63, 3523-43.

Bac-Molenaar, J. A., Granier, C., Keurentjes, J. J. and Vreugdenhil, D. (2016). Genome-wide association mapping of time-dependent growth responses to moderate drought stress in Arabidopsis. Plant Cell Environ 39, 88-102.

Barrero, J. M., RodríGuez, P. L., Quesada, V., Piqueras, P., Ponce, M. R. and Micol, J. L. (2006). Both abscisic acid (ABA)-dependent and ABA-independent pathways govern the induction of NCED3, AAO3 and ABA1 in response to salt stress. Plant, Cell \& Environment 29, 2000-2008.

Bouchabke, O., Chang, F., Simon, M., Voisin, R., Pelletier, G. and DurandTardif, M. (2008). Natural variation in Arabidopsis thaliana as a tool for highlighting differential drought responses. PLOS ONE 3, e1705.

Bu, Q., Jiang, H., Li, C. B., Zhai, Q., Zhang, J., Wu, X., Sun, J., Xie, Q. and $\mathbf{L i}, \mathbf{C}$. (2008). Role of the Arabidopsis thaliana NAC transcription factors ANAC019 and ANAC055 in regulating jasmonic acid-signaled defense responses. Cell Res 18, 756-67.

Cheng, M.-C., Liao, P.-M., Kuo, W.-W. and Lin, T.-P. (2013). The Arabidopsis ETHYLENE RESPONSE FACTOR1 Regulates Abiotic Stress-Responsive Gene Expression by Binding to Different cis-Acting Elements in Response to Different Stress Signals. Plant Physiol 162, 1566-1582.

De Keersmaecker, S. C., Varszegi, C., van Boxel, N., Habel, L. W., Metzger, K., Daniels, R., Marchal, K., De Vos, D. and Vanderleyden, J. (2005). Chemical synthesis of (S)-4,5-dihydroxy-2,3-pentanedione, a bacterial signal molecule precursor, and validation of its activity in Salmonella typhimurium. J Biol Chem 280, 19563-8.

Delauney, A. J. and Verma, D. P. S. (1993). Proline biosynthesis and osmoregulation in plants. The Plant Journal 4, 215-223.

Dombrecht, B., Xue, G. P., Sprague, S. J., Kirkegaard, J. A., Ross, J. J., Reid, J. B., Fitt, G. P., Sewelam, N., Schenk, P. M., Manners, J. M. et al. (2007). MYC2 differentially modulates diverse jasmonate-dependent functions in Arabidopsis. Plant Cell 19, 2225-45.

Fujita, M., Fujita, Y., Maruyama, K., Seki, M., Hiratsu, K., Ohme-Takagi, M., Tran, L. S., Yamaguchi-Shinozaki, K. and Shinozaki, K. (2004). A dehydrationinduced NAC protein, RD26, is involved in a novel ABA-dependent stress-signaling pathway. Plant J 39, 863-76.

Harb, A., Krishnan, A., Ambavaram, M. M. and Pereira, A. (2010). Molecular and physiological analysis of drought stress in Arabidopsis reveals early responses leading to acclimation in plant growth. Plant Physiol 154, 1254-71.

Heymann, H. and Noble, A. C. (1989). Comparison of Canonical Variate and Principal Component Analyses of Wine Descriptive Analysis Data. Journal of Food Science 54, 1355-1358.

Hickman, R., Hill, C., Penfold, C. A., Breeze, E., Bowden, L., Moore, J. D., Zhang, P., Jackson, A., Cooke, E., Bewicke-Copley, F. et al. (2013). A local regulatory network around three NAC transcription factors in stress responses and senescence in Arabidopsis leaves. Plant J 75, 26-39.

Hirt, H. (2004). Introduction. In Plant Responses to Abiotic Stress, vol. 4, pp. 1-8: Springer Berlin / Heidelberg.

Jensen, M. K., Kjaersgaard, T., Nielsen, M. M., Galberg, P., Petersen, K., O'Shea, C. and Skriver, K. (2010). The Arabidopsis thaliana NAC transcription factor 
family: structure-function relationships and determinants of ANAC019 stress signalling. Biochem J 426, 183-96.

Jiang, C.-J., Aono, M., Tamaoki, M., Maeda, S., Sugano, S., Mori, M. and Takatsuji, H. (2007). SAZ, a new SUPERMAN-like protein, negatively regulates a subset of ABA-responsive genes in Arabidopsis. Molecular Genetics and Genomics 279, 183-192. 686-703.

Kazan, K. and Manners, J. M. (2013). MYC2: the master in action. Mol Plant 6,

Kim, J., Malladi, A. and van Iersel, M. W. (2012). Physiological and molecular responses to drought in Petunia: the importance of stress severity. J Exp Bot 63, 6335-45.

Kim, S., Kang, J. Y., Cho, D. I., Park, J. H. and Kim, S. Y. (2004). ABF2, an ABRE-binding bZIP factor, is an essential component of glucose signaling and its overexpression affects multiple stress tolerance. Plant $\mathrm{J}$ 40, 75-87.

Kim, S. H., Woo, D. H., Kim, J. M., Lee, S. Y., Chung, W. S. and Moon, Y. H. (2011). Arabidopsis MKK4 mediates osmotic-stress response via its regulation of MPK3 activity. Biochem Biophys Res Commun 412, 150-4.

Kimura, Y., Aoki, S., Ando, E., Kitatsuji, A., Watanabe, A., Ohnishi, M., Takahashi, K., Inoue, S., Nakamichi, N., Tamada, Y. et al. (2015). A flowering integrator, SOC1, affects stomatal opening in Arabidopsis thaliana. Plant Cell Physiol 56, 640-9.

Kliebenstein, D. J., West, M. A., van Leeuwen, H., Kim, K., Doerge, R. W., Michelmore, R. W. and St Clair, D. A. (2006). Genomic survey of gene expression diversity in Arabidopsis thaliana. Genetics 172, 1179-89.

Lasky, J. R., Des Marais, D. L., Lowry, D. B., Povolotskaya, I., McKay, J. K., Richards, J. H., Keitt, T. H. and Juenger, T. E. (2014). Natural variation in abiotic stress responsive gene expression and local adaptation to climate in Arabidopsis thaliana. Mol Biol Evol 31, 2283-96.

Lee, S. J., Kang, J. Y., Park, H. J., Kim, M. D., Bae, M. S., Choi, H. I. and Kim, S. Y. (2010). DREB2C interacts with ABF2, a bZIP protein regulating abscisic acidresponsive gene expression, and its overexpression affects abscisic acid sensitivity. Plant Physiol 153, 716-27.

Li, Y., Huang, Y., Bergelson, J., Nordborg, M. and Borevitz, J. O. (2010). Association mapping of local climate-sensitive quantitative trait loci in Arabidopsis thaliana. Proc Natl Acad Sci U S A 107, 21199-204.

Loudet, O., Chaillou, S., Krapp, A. and Daniel-Vedele, F. (2003). Quantitative Trait Loci Analysis of Water and Anion Contents in Interaction With Nitrogen Availability in Arabidopsis thaliana. Genetics 163, 711-722.

Michaels, S. D. and Amasino, R. M. (1999). FLOWERING LOCUS C Encodes a Novel MADS Domain Protein That Acts as a Repressor of Flowering. Plant Cell 11, 949-956.

Miller, M., Song, Q., Shi, X., Juenger, T. E. and Chen, Z. J. (2015). Natural variation in timing of stress-responsive gene expression predicts heterosis in intraspecific hybrids of Arabidopsis. 6.

Nakashima, K., Yamaguchi-Shinozaki, K. and Shinozaki, K. (2014). The transcriptional regulatory network in the drought response and its crosstalk in abiotic stress responses including drought, cold, and heat. Frontiers in Plant Science 5, 170.

Okuma, E., Nozawa, R., Murata, Y. and Miura, K. (2014). Accumulation of endogenous salicylic acid confers drought tolerance to Arabidopsis. Plant Signal Behav 9, e28085.

Onate-Sanchez, L. and Vicente-Carbajosa, J. (2008). DNA-free RNA isolation protocols for Arabidopsis thaliana, including seeds and siliques. BMC Res Notes 1, 93.

Pareek, A., Sopory, S. K., Bohnert, H. J. and Govindjee. (2010). Abiotic Stress Adaptation in Plants : Physiological, Molecular and Genomic Foundation. Dordrecht: Springer Science+Business Media B.V.

Pieterse, C. M., Van der Does, D., Zamioudis, C., Leon-Reyes, A. and Van Wees, S. C. (2012). Hormonal modulation of plant immunity. Annu Rev Cell Dev Biol 28, 489-521. 
Prasch, C. M. and Sonnewald, U. (2013). Simultaneous application of heat, drought, and virus to Arabidopsis plants reveals significant shifts in signaling networks. Plant Physiol 162, 1849-66.

Qin, F., Sakuma, Y., Tran, L.-S. P., Maruyama, K., Kidokoro, S., Fujita, Y., Fujita, M., Umezawa, T., Sawano, Y., Miyazono, K.-i. et al. (2008a). Arabidopsis DREB2A-Interacting Proteins Function as RING E3 Ligases and Negatively Regulate Plant Drought Stress-Responsive Gene Expression. Plant Cell 20, 1693-1707.

Qin, F., Sakuma, Y., Tran, L. S. P., Maruyama, K., Kidokoro, S., Fujita, Y., Fujita, M., Umezawa, T., Sawano, Y., Miyazono, K. I. et al. (2008b). Arabidopsis DREB2A-interacting proteins function as RING E3 ligases and negatively regulate plant drought stress-responsive gene expression. Plant Cell 20, 1693-1707.

Rai, A. K. and Takabe, T. (2006). Abiotic stress tolerance in plants : toward the improvement of global environment and food. Dordrecht: Springer.

Rasmussen, S., Barah, P., Suarez-Rodriguez, M. C., Bressendorff, S., Friis, P., Costantino, P., Bones, A. M., Nielsen, H. B. and Mundy, J. (2013). Transcriptome responses to combinations of stresses in Arabidopsis. Plant Physiol 161, 1783-94.

Robert-Seilaniantz, A., Bari, R. and Jones, J. D. G. (2010). A Biotic or Abiotic Stress? In Abiotic Stress Adaptation in Plants, eds. A. Pareek S. K. Sopory H. J. Bohnert and Govindjee), pp. 103-122: Springer Netherlands.

Savoure, A., Hua, X. J., Bertauche, N., Van Montagu, M. and Verbruggen, N. (1997). Abscisic acid-independent and abscisic acid-dependent regulation of proline biosynthesis following cold and osmotic stresses in Arabidopsis thaliana. Mol Gen Genet 254, 104-9.

Shang, Y., Yan, L., Liu, Z.-Q., Cao, Z., Mei, C., Xin, Q., Wu, F.-Q., Wang, X.F., Du, S.-Y., Jiang, T. et al. (2010). The Mg-Chelatase H Subunit of Arabidopsis Antagonizes a Group of WRKY Transcription Repressors to Relieve ABA-Responsive Genes of Inhibition. Plant Cell 22, 1909-1935.

Shinozaki, K. and Yamaguchi-Shinozaki, K. (2007). Gene networks involved in drought stress response and tolerance. J Exp Bot 58, 221-7.

Sokal RR, R. F. (1981). Biometry the Principles and Practice of Statistics in Biological Research (2nd ed.). . WH Freeman and Company, San Francisco, USA.

Strizhov, N., Abraham, E., Okresz, L., Blickling, S., Zilberstein, A., Schell, J., Koncz, C. and Szabados, L. (1997). Differential expression of two P5CS genes controlling proline accumulation during salt-stress requires $A B A$ and is regulated by $A B A 1$, ABI1 and AXR2 in Arabidopsis. Plant J 12, 557-69.

Suzuki, N., Rivero, R. M., Shulaev, V., Blumwald, E. and Mittler, R. (2014). Abiotic and biotic stress combinations. New Phytol 203, 32-43.

Szekely, G., Abraham, E., Cseplo, A., Rigo, G., Zsigmond, L., Csiszar, J., Ayaydin, F., Strizhov, N., Jasik, J., Schmelzer, E. et al. (2008). Duplicated P5CS genes of Arabidopsis play distinct roles in stress regulation and developmental control of proline biosynthesis. Plant J 53, 11-28.

Takahashi, H., Kanayama, Y., Zheng, M. S., Kusano, T., Hase, S., Ikegami, M. and Shah, J. (2004). Antagonistic Interactions between the SA and JA Signaling Pathways in Arabidopsis Modulate Expression of Defense Genes and Gene-for-Gene Resistance to Cucumber Mosaic Virus. Plant and Cell Physiology 45, 803-809.

Uno, Y., Furihata, T., Abe, H., Yoshida, R., Shinozaki, K. and YamaguchiShinozaki, K. (2000). Arabidopsis basic leucine zipper transcription factors involved in an abscisic acid-dependent signal transduction pathway under drought and high-salinity conditions. Proc Natl Acad Sci U S A 97, 11632-7.

Van Poecke, R. M. P., Sato, M., Lenarz-Wyatt, L., Weisberg, S. and Katagiri, F. (2007). Natural Variation in RPS2-Mediated Resistance among Arabidopsis Accessions: Correlation between Gene Expression Profiles and Phenotypic Responses. Plant Cell 19, 4046-4060.

Van Wees, S. C. M., Van Pelt, J. A., Bakker, P. A. H. M. and Pieterse, C. M. J. (2013). Bioassays for assessing jasmonate-dependent defenses triggered by pathogens, herbivorous Insects, or beneficial rhizobacteria. Methods in Molecular Biology 1011, 35-49. 
Verslues, P. E., Agarwal, M., Katiyar-Agarwal, S., Zhu, J. and Zhu, J. K. (2006). Methods and concepts in quantifying resistance to drought, salt and freezing, abiotic stresses that affect plant water status. Plant J 45, 523-39.

Verslues, P. E. and Sharma, S. (2010). Proline Metabolism and Its Implications for Plant-Environment Interaction. Arabidopsis Book 8, e0140.

Vos, I. A., Verhage, A., Schuurink, R. C., Watt, L. G., Pieterse, C. M. J. and Van Wees, S. C. M. (2013). Onset of herbivore-induced resistance in systemic tissue primed for jasmonate-dependent defenses is activated by abscisic acid. Frontiers in Plant Science 4, 539.

Wang, Y., Liu, C., Li, K., Sun, F., Hu, H., Li, X., Zhao, Y., Han, C., Zhang, W., Duan, Y. et al. (2007). Arabidopsis EIN2 modulates stress response through abscisic acid response pathway. Plant Mol Biol 64, 633-644.

Xiong, L. and Ishitani, M. (2006). Stress signal transduction: components, pathways and network integration. In Abiotic stress tolerance in plants, eds. A. K. Rai and T. Takabe), pp. 3-29: Springer Netherlands.

Xiong, L., Lee, H., Ishitani, M. and Zhu, J. K. (2002). Regulation of osmotic stress-responsive gene expression by the LOS6/ABA1 locus in Arabidopsis. J Biol Chem 277, 8588-96.

Xiong, L. and Zhu, J. K. (2003). Regulation of abscisic acid biosynthesis. Plant Physiol 133, 29-36.

Xu, X., Chen, C., Fan, B. and Chen, Z. (2006). Physical and Functional Interactions between Pathogen-Induced Arabidopsis WRKY18, WRKY40, and WRKY60 Transcription Factors. Plant Cell 18, 1310-1326.

Yamaguchi-Shinozaki, K. and Shinozaki, K. (1993). The plant hormone abscisic acid mediates the drought-induced expression but not the seed-specific expression of rd22, a gene responsive to dehydration stress in Arabidopsis thaliana. Mol Gen Genet 238, 17-25.

Yamaguchi-Shinozaki, K. and Shinozaki, K. (1994). A novel cis-acting element in an Arabidopsis gene is involved in responsiveness to drought, low-temperature, or high-salt stress. Plant Cell 6, 251-64.

Yoo, J. H., Park, C. Y., Kim, J. C., Heo, W. D., Cheong, M. S., Park, H. C., Kim, M. C., Moon, B. C., Choi, M. S., Kang, Y. H. et al. (2005). Direct interaction of a divergent CaM isoform and the transcription factor, MYB2, enhances salt tolerance in arabidopsis. J Biol Chem 280, 3697-706.

Yoshioka, K. and Shinozaki, K. (2009). Signal Crosstalk in Plant Stress Responses: Wiley.

Zelditch, M., Swiderski, D. and Sheets, H. D. (2012). Geometric Morphometrics for Biologists: A Primer: Elsevier Academic Press. 


\section{Supporting Information}

Additional supporting information may be download via link

https://www.dropbox.com/sh/okm057i3t7xmbvy/AACYpZRnIMJCYxQHPnD-Z41-a?dl=0 



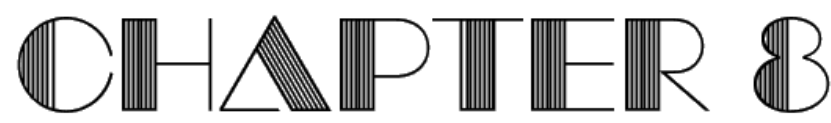

General discussion

Pingping Huang 
This thesis deals with the responses of Arabidopsis thaliana (Arabidopsis) plants to sequential combinations of biotic and drought stress triggers, with the identification of relevant regulatory genes as its main objective. Pieris rapae and Botrytis cinerea were employed as biotic stress activators. Following the biological treatment(s), the investigated plants were subjected to abiotic stress conditions (drought). Both Pieris and Botrytis trigger expression of defenseresponsive genes from the jasmonate (JA) signalling pathway; however, the respective defense mechanisms mostly follow alternative courses. The Pieris response is mediated mainly through the MYC pathway branch, whereas Botrytis infection, activates the ethylene response factor (ERF) branch (Dombrecht et al., 2007). The two sub-networks can interact with each other antagonistically. Hence, some JA-ET-responsive genes were expected to be shared in both investigated biotic stress mechanisms but others might show opposite effects. In addition, the MYC and ERF branches engage, respectively, in positive and negative interactions with the drought-induced abscisic acid ( $A B A)$ signalling pathway (Dombrecht et al., 2007). Therefore, the hypothesis that the JA-ETresponsive genes and therefore also Pieris and Botrytis pre-treatments exert contrasting influence on the ABA-mediated drought stress response was addressed. Transcriptiome analysis identifed 31 genes, which exhibited opposite directions of expression response when comparing the two combinatorial stresses, and indeed found differential regulation of several plant hormonerelated genes such as ERF4, ERF5, ERF6 and ERF104 (Chapter 6).

\section{GWA-mapping and RNA-seq}

Two genetic approaches were used to study the effects of sequential stress combinations in plants in comparison with single factor responses and to identify genes relevant especially to the combinatorial stress mechanisms.

RNA sequencing (RNA-seq) using the Next Generation Sequencing approach is a straightforward technique affording identification of genes showing differential transcription levels under various conditions. Next to determining levels of transcription, RNA-seq will reveal alternative splicing (AS) variants influenced by diverse environments. This can be interesting, as for instance analysis of AS variants of the ZINC-INDUCED FACILITATOR1 (ZIFL1) gene 
showed that two of its isoforms (ZIFL1.1 and ZIFL1.3) exhibited distinct localization and played different roles, either to modulate root polar auxin transport by localizing to the root cell tonoplasts (ZIFL1.1), or to affect drought tolerance when expressed in stomatal guard cells and localised to the plasma membrane (ZIFL1.3) (Remy et al., 2013). In the response to combinatorial stresses, AS variants of the auxin efflux carrier PIN7 were found. The AS variant PIN7-1 exhibited enhanced expression compared to PIN7-2 under the stress combination Pieris and subsequent drought in comparison to the single drought treatment, suggesting that this particular isoform is more relevant in the response to the combinatorial stress than the other isoform (Chapter 6).

Genome-wide association (GWA) analysis identifies genes for which there is genetic variation that leads to differences in the genotype's response to treatments while RNA-seq, using a single genotype, identifies genes that respond in expression to the treatments. While gene expression differences are very interesting to identify genes that play a role in environmental responses, it will not reveal anything on the genetic variation for environmental response, that can be illustrative for breeders to focus on when trying to improve crop robustness and tolerance to environmental fluctuations. However, some overlap was found between the genes identified in both approaches. For instance, in case of the effect of Pieris and subsequent drought stress response, one identified quantitative trait locus (QTL) overlapped with differential expression of two genes, PRX34 and AT3G49350 (Chapters $3 \& 6$ ), while for Botrytis infection, a similar overlap was seen for gene AT3G02910 (Chapters 3 \& 6).

The GWA-mapping approach affords discerning connections between DNA sequence polymorphisms and their phenotypic consequences. Genetic variation can either exert its impact through single-nucleotide polymorphisms (SNPs) associated with gene transcription differences or those affecting protein functions. For instance, Arabidopsis natural accessions carrying a single SNP at the HIGH-AFFINITY $K+$ TRANSPORTER $1 ; 1(A t H K T 1 ; 1)$ gene showed an aberrant expression of the gene leading to a difference in leaf $\mathrm{Na}^{+}$accumulation (Baxter et al., 2010); and a single SNP substitution in CRY2 led to an amino acid substitution affecting CRY2 protein stability under short photoperiods, thus leading to early flowering in Arabidopsis thaliana (El-Assal et al., 2001). 
GWA-mapping has been used in many studies and proved successful in detecting SNPS causal to their corresponding phenotypic traits (Atwell et al., 2010; Bac-Molenaar et al., 2016; Meijon et al., 2014). The challenges of the technique were described by Weigel (2012) and include 1) false positives: the identified SNPS may not be the causal ones or the association may incorrectly identify SNPs as associated, 2) population structure: association may be found due to close relationships between genotypes, rather than shared SNPs based on short-range linkage disequilibrium, and 3) allelic heterogeneity: different SNPS at the same locus lead to similar phenotypes, but they will not be detected through GWA analysis (Barboza et al., 2013).

Using a Bonferroni-corrected threshold $(-\log (p)=6.5)$ to reduce false positives is advised by Atwell et al (2010). However, the Bonferroni corrected threshold is extremely conservative and often not convenient for growth related traits such as FW, DW, WC, and PLA because these traits are genetically influenced by a multitude of small-effect QTLs. An arbitrary threshold of $\log (p)=4$ as described by van Rooijen et al. (2015) was used to assign associated SNPs in Chapter 3 and Chapter 4, and a number of SNPs exhibited genetic variance in response to stresses were discovered, with associated genes for which mutants showed a relevant aberrant phenotype. Although the number of false positive associated SNPs will be larger than when the Bonferroni threshold is used, there will be sufficient SNPs found that are indeed associated with the phenotype. Especially for complex polygenic traits this seems to be a practically viable approach for GWAs analysis.

In many GWAs studies, statistical approaches were applied to correct population structure ( Yu et al., 2006; Atwell et al., 2010; Brachi et al., 2010). However, correcting population structure can increase the frequency of false negatives and led to miss the causative genetic markers (Brachi et al., 2010). GWA analysis in Chapter 2, 3, and 4, used the 350 accessions, which eliminated population structure based on testing results of 149 SNPs for genetic diversity (Li et al., 2010; Platt et al., 2010). In combination with mutant test, I confirmed the biological function of some SNPs associated candidate genes under different conditions. 
Several studies combined association mapping with linkage mapping, which helped substantially in identifying causal genetic variation eg. in case of flowering time (Brachi et al., 2010), resistance to Hyaloperonospora arabidopsidis ex parasitica (Hpa) (Nemri et al., 2010), endoreduplication (Sterken et al., 2012), or shoot regeneration (Motte et al., 2014). Thus, to confirm the loci associated with combinatorial stress response and to reveal false negatives, follow-up studies of QTL analysis using biparental populations might be useful. Although in my studies, the allelic effects of some genes on combinatorial stress responses could be highlighted, the physiological function of many allelic variants in the response to stress conditions remains to be discovered. Future experiments, such as gene expression studies aiming at determining the effects of SNP variation or different allelic groups on gene expression or complementation tests to examine the gain or loss of function effects of the SNPs or alternative types of alleles under diverse stress conditions, should be performed.

Using different trait values was also recommend for GWA analysis. For instance, residuals that represent the variations of the accessions (genotype) in response to stresses (environment) (Filiault and Maloof, 2012) are suitable for univariate GWA analysis (Chapter 3 \& 4). Principal component (PC) values are suitable for multi-trait multi-environment analysis (Chapter 2).

\section{GWA analysis identifies small-effects QTLS}

Moderate to high heritability was found for the measured traits in the single and combinatorial stress conditions, indicating the variation among accessions in their responses to stresses due to their genetic variation (Chapter $3 \& 4$ ). Normally, moderate to high heritability offers good prospects to identify QTLS affecting the trait. In my study, each identified individual QTL explained a small part of the genetic variance (on average 4-6\%). This is not a surprising because the traits we measured (FW, DW, WC and PLA) are very general growth traits, which are likely to be genetically affected by allelic variation at many loci. Few loci with large genetic variance were not able to be identified by GWAs analysis approach (Brachi et al; 2010). The large number of small-effect QTLs could make it very difficult to identify these QTLs (Kooke et al., 2016). Thus, the 
causal variants could be due either to a small number of genes, with relatively large effects; or due to many genes, with relatively small effects. Analysis of a simple trait often results in less QTLs, but with larger effect sizes, than for a complex trait (Louthan and Kay, 2011).

\section{Different models in GWA-mapping}

In the present study, the interactions of Arabidopsis accessions with an array of enviromental conditions were tested. Various phenotypic parameters were taken into account, and different models of GWA-mapping were applied to determine the effects of SNP variants on multiple stress responses. Models such as multi-trait QTL mixed models (MTMMs) (Chapter 2), Multi-environment (ME) analysis, , and univriate analysis (Chapter $3 \& 4$ ). Residuals resulting from the regression of the response to the combinaitonal stress on the response to single stress were used as trait values (Chapter $3 \& 4$ ).

A recent study, appying QTL analysis to investigate yield-related traits in pepper, showed a partial overlap of the identified significant QTLs when comparing MTME, ME, multi-trait (MT), and univariate single environment (SE) mapping approaches (Alimi et al., 2013). In my study, the comparison of ME GWA-mapping with univariate analysis resulted in identification of eight shared SNPs with QTL by environment interaction ( $Q \times E)$ effects of subsequent drought for those Pieris pre-challenged plants and four common SNPs with Q $\times E$ effects in subsequent drought stress response for those infected with Botrytis (Chapter 3 ). This is not a surprise because the univriate analysis used residuals which also represent QXE effects. The 12 SNPs that were identified by both approaches indicates these could be true causative QTLs. The partly overlap between the univariate and ME approahces was oversation found by El-Soda et al. (2015) as well, where a positive correlation between the -log10 values of the $214 \mathrm{k}$ SNPs resulted from two approaches were reported.

Korte et al. (2012) suggest that ME mapping using MTMM is more powerful than the univariate approach as it takes the variation within and between trait(s) into consideration, while the latter affords identification of SNPs associated with main effects only, without accounting for the $\mathrm{Q} \times \mathrm{E}$ interactions. However, as my 
study used residuals that represent genotype $x$ environment $(G \times E)$ variation for the univariate analysis, it does take at least some of the $\mathrm{Q} \times \mathrm{E}$ into consideration. Several studies reported limited efficacy of the ME GWA-mapping in detection of Q $\times$ E effects for strong QTLs (Bac-Molenaar et al., 2015; Korte et al., 2012; Morrison and Linder, 2014). In these studies, more significant SNPs were identified using the univariate method than when the ME mapping was applied. The same was the case in my studies. This does not mean the ME method is the less efficient one, as many of the SNPs identified in the univariate analysis could be false positives (Chapter 3). Thorough follow-up research would be needed to evaluate these results properly, but this is very time-consuming and labor intensive.

\section{Different interactions between biotic and abiotic}

\section{stress}

Abiotic stresses can significantly affect plant responses to biotic strains and vice versa (Atkinson and Urwin, 2012; Appel et al., 2014; Rejeb et al., 2014). The interaction between biotic and abiotic stress responses is often mediated by different plant hormones and the outcome can be synergistic, antagonistic, or neutral (Audenaert et al., 2002; Achuo et al., 2006; Suzuki et al., 2014; Ramegowda and Senthil-Kumar, 2015; Prasch and Sonnewald, 2015). In Chapter 3, 4, 6 and 7, different phenotypic and transcriptomic responses to the single and combinatorial stress were observed. For instance, we found that the biotic pre-treatment promotes the susceptibility of plants to drought stress. For instance, by reducing rosette dry weight (DW) during sequential drought, but increasing the water content (WC) (Chapter 7). Three types of interaction between biotic and abiotic stress were observed. For example, neutral effects were found of the interaction between Pieris and osmotic stress on bzip25 mutants, antagonistic effects were found between Pieris and osmotic stress on the prx34 mutant and between Botrytis and osmotic stress in the rmg1 mutant (Chapter 3), while a synergistic effect was observed between drought and Botrytis in the pal4 mutant (Chapter 4). 


\section{Combinatorial stress responses cannot be predicted by comparing single stress responses}

Ramegowda and Senthil-Kumar (2015) suggest there has to be a unique response to combinatorial stresses as the responses of plants to blends of triggering factors cannot be predicted based on mechanisms responding to their individual components. For instance, $61 \%$ of the changes in gene expression in response to the combinatorial stress could not be predicted from the single stress responses (Rasmussen et al., 2013). Transcriptome analysis of simultaneous effects of drought, heat, and virus infections in Arabidopsis identified only 11 genes involved in all single, double, and triple stress treatments (Prasch and Sonnewald, 2013). This is not much, considering Arabidopsis has around 30,000 genes, with often at least half of them expressed at any time point in any organ.

Genes involved in multiple stress responses may not be able to identified under single stress conditions. For instance, the expression of the resistance gene RPS6 was only regulated in case of simultaneous heat, drought, and virus treatment, but not under any of the double or individual stresses (Prasch and Sonnewald, 2013). In my study, when comparing the Botrytis and subsequent drought with single drought and Botrytis treatments, $4 \%$ of DEGs identified as Botrytis and subsequent drought responsive genes were not induced by either single Botrytis or drought treatments; comparing DEGs from the stress combination of Pieris and subsequent drought with single drought and Pieris treatments, $3 \%$ of DEGs identified as Pieris and subsequent drought responsive genes were not induced by either Pieris or drought (Chapter 6).

The aim to study combinatorial stress responses is to understand how plants react in a close to natural environment, as a combinatorial stress is more likely to occur in a natural environment than a single stress. Transcriptome analysis identified several combinatorial stress responsive genes that cannot be induced by neither by the biotic nor drought, showing that these combinatorial stress responsive genes could be the potential candidate genes for plant breeding use. 


\section{Experimental design}

The combinatorial treatment in the GWA-mapping experiments was slightly different from the one used for the RNA-seq analysis, with a $24 \mathrm{~h}$ recovery period included in between the first stress condition and the sequential drought in case of the GWA-mapping (Chapter 3, Supplementary Figure 1). As the interaction between Pieris and drought and between Botrytis and drought on plants could be observed for the expression of bZIP25 and PRX34 (for Pieris \& drought) and for Di19 and WRR4 (for Botrytis \& drought) under combinatorial treatment in comparison to single drought treatment (Chapter 3), I consider the $24 \mathrm{~h}$ recovery period still acceptable for observing the interaction between the first challenge and the sequential drought stress response.

The investigated plants differed in recovery speed from the Botrytis and Pieris treatments. For instance, the RNA-seq analysis reported a much lower number of DEGs in the Botrytis-challenged plants following subsequent drought treatment as compared to those subjected to initial Pieris herbivory and subsequent drought. This observation could stem from the fast recovery of plants from the exposure to Botrytis, with few residual infection-induced signals at the time of the application of drought. In order to get a stronger interaction between Botrytis and drought, it will be better to apply a quick induction of drought in plants. Such could be achieved by applying polyethylene glycol (PEG), which will emphasize the osmotic component of drought, or to ensure that moisture evaporates faster from soil eg. by increasing air circulation.

The impact of the first stress treatment on plant response to sequential ones can be strong but depends on the nature of the initial challenge (Chapter 5). While prior herbivory strongly affected Botrytis resistance (Coolen, 2016), we identified six times more DEGs under the Pieris and subsequent drought than under Botrytis and subsequent drought, showing a stronger effect of prior Pieris exposure on gene expression than prior Botrytis infection (Chapter 6). However, this also could mean that Pieris herbivory is more severe than Botrytis infection. For instance, Windram et al (2012)) used the same concentration of Botrytis spores as in this study, and showed that the first symptom of Botrytis infection on leaf can be observed at $20 \mathrm{~h}$ after inoculation (HAI) and the expansion of 
lesions at 36 HAI. In order to have a stronger effect of Botrytis infection, a higher spore concentration (e.g. using spore concentration at $1 \times 10^{6}$ per $\mathrm{ml}$ ), increase the number of droplets per leaf, or to inoculate Botrytis spores on plants in the late afternoon (e.g. inoculate at 18 hour) (Ingle et al., 2015) could be used.

\section{Conclusions and recommendations}

The research described in this thesis provides insights into plant response mechanisms triggered by sequential combinations of biotic and drought stress factors, facilitating our understanding of the intricate network of defense processes in planta. A number of candidate genes to combinatorial stress responses were identified by GWA analysis and RNA-seq. The physiological function of some candidate genes in different stress conditions were characterized using T-DNA insertion mutants and gene expression study. However, the physiological function of many allelic variants in stress conditions remain to be discovered. The present study provides an important step towards in-depth understanding of plant responses to the investigated stress factors and highlights the importance of an array of genes, crucial to the underlying defense processes, as targets for breeding by allele mining aimed at ultimate improvement of crop tolerance to relevant stress factor combinations. 


\section{References}

Achuo, E. A., Prinsen, E. and Höfte, M. (2006). Influence of drought, salt stress and abscisic acid on the resistance of tomato to Botrytis cinerea and Oidium neolycopersici Plant Pathology Volume 55, Issue 2. In Plant Pathology, vol. 55, pp. 178186.

Alimi, N. A., Bink, M. C., Dieleman, J. A., Magan, J. J., Wubs, A. M., Palloix, A. and van Eeuwijk, F. A. (2013). Multi-trait and multi-environment QTL analyses of yield and a set of physiological traits in pepper. Theor Appl Genet 126, 2597-625.

Appel, H. M., Maqbool, S. B., Raina, S., Jagadeeswaran, G., Acharya, B. R., Hanley, J. C., Miller, K. P., Hearnes, L., Jones, A. D., Raina, R. et al. (2014). Transcriptional and metabolic signatures of Arabidopsis responses to chewing damage by an insect herbivore and bacterial infection and the consequences of their interaction. Frontiers in Plant Science 5, 441.

Atkinson, N. J. and Urwin, P. E. (2012). The interaction of plant biotic and abiotic stresses: from genes to the field. Journal of Experimental Botany 63, 3523-3543.

Atwell, S., Huang, Y. S., Vilhjalmsson, B. J., Willems, G., Horton, M., Li, Y., Meng, D., Platt, A., Tarone, A. M., Hu, T. T. et al. (2010). Genome-wide association study of 107 phenotypes in Arabidopsis thaliana inbred lines. Nature 465, 627-31.

Audenaert, K., De Meyer, G. B. and Hofte, M. M. (2002). Abscisic acid determines basal susceptibility of tomato to Botrytis cinerea and suppresses salicylic aciddependent signaling mechanisms. Plant Physiol 128, 491-501.

Bac-Molenaar, J. A., Fradin, E. F., Becker, F. F., Rienstra, J. A., van der Schoot, J., Vreugdenhil, D. and Keurentjes, J. J. (2015). Genome-Wide Association Mapping of Fertility Reduction upon Heat Stress Reveals Developmental Stage-Specific QTLs in Arabidopsis thaliana. Plant Cell 27, 1857-74.

Bac-Molenaar, J. A., Granier, C., Keurentjes, J. J. and Vreugdenhil, D. (2016). Genome-wide association mapping of time-dependent growth responses to moderate drought stress in Arabidopsis. Plant Cell Environ 39, 88-102.

Barboza, L., Effgen, S., Alonso-Blanco, C., Kooke, R., Keurentjes, J. J., Koornneef, M. and Alcazar, R. (2013). Arabidopsis semidwarfs evolved from independent mutations in GA20ox1, ortholog to green revolution dwarf alleles in rice and barley. Proc Natl Acad Sci U S A 110, 15818-23.

Baxter, I., Brazelton, J. N., Yu, D., Huang, Y. S., Lahner, B., Yakubova, E., Li, Y., Bergelson, J., Borevitz, J. O., Nordborg, M. et al. (2010). A coastal cline in sodium accumulation in Arabidopsis thaliana is driven by natural variation of the sodium transporter AtHKT1;1. PLoS Genet 6, e1001193.

Brachi, B., Faure, N., Horton, M., Flahauw, E., Vazquez, A., Nordborg, M., Bergelson, J., Cuguen, J. and Roux, F. (2010). Linkage and association mapping of Arabidopsis thaliana flowering time in nature. PLoS Genet 6, e1000940.

Coolen, S. P., S; Hickman, R; Davila Olivas, N; Huang, P; Van Verk, M; Van Pelt, J; Wittenberg, A; De Vos, M; Prins, M; van Loon, J; Aarts, M; Dicke, M; Pieterse, C; van Wees, S (2016). Transcriptome dynamics of Arabidopsis during sequential biotic and abiotic stresses. The Plant Journal.

Dombrecht, B., Xue, G. P., Sprague, S. J., Kirkegaard, J. A., Ross, J. J., Reid, J. B., Fitt, G. P., Sewelam, N., Schenk, P. M., Manners, J. M. et al. (2007). MYC2 differentially modulates diverse jasmonate-dependent functions in Arabidopsis. Plant Cell 19, 2225-45.

El-Assal, S., Alonso-Blanco, C., Peeters, A. J., Raz, V. and Koornneef, M. (2001). A QTL for flowering time in Arabidopsis reveals a novel allele of CRY2. Nat Genet 29, 435-40.

El-Soda, M., Kruijer, W., Malosetti, M., Koornneef, M. and Aarts, M. G. (2015). Quantitative trait loci and candidate genes underlying genotype by environment interaction in the response of Arabidopsis thaliana to drought. Plant Cell Environ 38, 58599. 
Filiault, D. L. and Maloof, J. N. (2012). A genome-wide association study identifies variants underlying the Arabidopsis thaliana shade avoidance response. PLoS Genet 8, e1002589.

Ingle, R. A., Stoker, C., Stone, W., Adams, N., Smith, R., Grant, M., Carré, I., Roden, L. C. and Denby, K. J. (2015). Jasmonate signalling drives time-of-day differences in susceptibility of Arabidopsis to the fungal pathogen Botrytis cinerea. The Plant Journal 84, 937-948.

Korte, A., Vilhjalmsson, B. J., Segura, V., Platt, A., Long, Q. and Nordborg, M. (2012). A mixed-model approach for genome-wide association studies of correlated traits in structured populations. Nat Genet 44, 1066-71.

Li, Y., Huang, Y., Bergelson, J., Nordborg, M. and Borevitz, J. O. (2010). Association mapping of local climate-sensitive quantitative trait loci in Arabidopsis thaliana. Proc Natl Acad Sci U S A 107, 21199-204.

Louthan, A. M. and Kay, K. M. (2011). Comparing the adaptive landscape across trait types: larger QTL effect size in traits under biotic selection. BMC Evolutionary Biology 11, 1-12.

Meijon, M., Satbhai, S. B., Tsuchimatsu, T. and Busch, W. (2014). Genomewide association study using cellular traits identifies a new regulator of root development in Arabidopsis. 46, 77-81.

Morrison, G. D. and Linder, C. R. (2014). Association mapping of germination traits in Arabidopsis thaliana under light and nutrient treatments: searching for GxE effects. G3 (Bethesda) 4, 1465-78.

Motte, H., Vercauteren, A., Depuydt, S., Landschoot, S., Geelen, D., Werbrouck, S., Goormachtig, S., Vuylsteke, M. and Vereecke, D. (2014). Combining linkage and association mapping identifies RECEPTOR-LIKE PROTEIN KINASE1 as an essential Arabidopsis shoot regeneration gene. Proc Natl Acad Sci U S A 111, 8305-10.

Nemri, A., Atwell, S., Tarone, A. M., Huang, Y. S., Zhao, K., Studholme, D. J., Nordborg, M. and Jones, J. D. (2010). Genome-wide survey of Arabidopsis natural variation in downy mildew resistance using combined association and linkage mapping. Proc Natl Acad Sci U S A 107, 10302-7.

Platt, A., Horton, M., Huang, Y. S., Li, Y., Anastasio, A. E., Mulyati, N. W., Agren, J., Bossdorf, O., Byers, D., Donohue, K. et al. (2010). The scale of population structure in Arabidopsis thaliana. PLoS Genet 6, e1000843.

Prasch, C. M. and Sonnewald, U. (2013). Simultaneous application of heat, drought, and virus to Arabidopsis plants reveals significant shifts in signaling networks. Plant Physiol 162, 1849-66.

Prasch, C. M. and Sonnewald, U. (2015). Signaling events in plants: Stress factors in combination change the picture. Environmental and Experimental Botany 114, 4-14.

Ramegowda, V. and Senthil-Kumar, M. (2015). The interactive effects of simultaneous biotic and abiotic stresses on plants: mechanistic understanding from drought and pathogen combination. Journal of Plant Physiology 176, 47-54.

Rasmussen, S., Barah, P., Suarez-Rodriguez, M. C., Bressendorff, S., Friis, P., Costantino, P., Bones, A. M., Nielsen, H. B. and Mundy, J. (2013). Transcriptome Responses to Combinations of Stresses in Arabidopsis. Plant Physiology 161, 1783-1794.

Rejeb, I. B., Pastor, V. and Mauch-Mani, B. (2014). Plant responses to simultaneous biotic and abiotic stress: molecular mechanisms. Plants 3, 458-475.

Remy, E., Cabrito, T. R., Baster, P., Batista, R. A., Teixeira, M. C., Friml, J., Sá-Correia, I. and Duque, P. (2013). A Major Facilitator Superfamily Transporter Plays a Dual Role in Polar Auxin Transport and Drought Stress Tolerance in Arabidopsis. Plant Cell 25, 901-926.

Sterken, R., Kiekens, R., Boruc, J., Zhang, F., Vercauteren, A., Vercauteren, I., De Smet, L., Dhondt, S., Inze, D., De Veylder, L. et al. (2012). Combined linkage and association mapping reveals CYCD5;1 as a quantitative trait gene for endoreduplication in Arabidopsis. Proc Natl Acad Sci U S A 109, 4678-83.

Suzuki, N., Rivero, R. M., Shulaev, V., Blumwald, E. and Mittler, R. (2014). Abiotic and biotic stress combinations. New Phytol 203, 32-43. 
van Rooijen, R., Aarts, M. G. and Harbinson, J. (2015). Natural genetic variation for acclimation of photosynthetic light use efficiency to growth irradiance in Arabidopsis. Plant Physiol 167, 1412-29.

Weigel, D. (2012). Natural variation in Arabidopsis: from molecular genetics to ecological genomics. Plant Physiol 158, 2-22.

Windram, O., Madhou, P., McHattie, S., Hill, C., Hickman, R., Cooke, E., Jenkins, D. J., Penfold, C. A., Baxter, L., Breeze, E. et al. (2012). Arabidopsis defense against Botrytis cinerea: chronology and regulation deciphered by high-resolution temporal transcriptomic analysis. Plant Cell 24, 3530-57.

Yu, J., Pressoir, G., Briggs, W. H., Vroh Bi, I., Yamasaki, M., Doebley, J. F., McMullen, M. D., Gaut, B. S., Nielsen, D. M., Holland, J. B. et al. (2006). A unified mixed-model method for association mapping that accounts for multiple levels of relatedness. Nat Genet 38, 203-8. 



\section{Summary in English}

Biotic and abiotic stresses are often occurring either simultaneous or consecutively in the natural environment and the impact of the two types of stresses on plants are often negative. Abiotic stresses can significantly affect plant responses to biotic stresses and vice versa. In the research described in this thesis, Arabidopsis thaliana was used as a model to study plant responses to different sequential combinations of biotic factors (infection with Botrytis cinerea or herbivory by Pieris rapae) and drought. Genome-wide association (GWA) mapping and RNA sequencing (RNA-seq) approaches were used to identify combinatorial stress responsive genes. The main objective of the research described in this thesis is to identify genes that contribute to tolerance to the aforementioned sequential stress combinations.

In Chapter 2, I describe multi-trait QTL mixed models (MTMMs) GWAmapping with traits from 11 single environmental factors and several of their consecutive combinations. Thirty significant SNPs that associated with genes responding to multiple stresses were identified and the average QTL-effect-sizes were found to be stronger for combinatorial stresses than single stresses. This is showing that plants appear to deploy broad-spectrum defensive mechanisms influencing multiple traits in response to combined stresses. Association analyses were performed to identify QTLs contributing to contrasting and similar responses to biotic versus abiotic stresses and belowground versus aboveground stresses. Candidate genes such as RMG1 and WRKY38 were identified in response to multiple environments and found to exhibit contrasting responses to biotic and abiotic stress. The rmg1 mutant was more tolerant to Pieris herbivory and more susceptible to osmotic and salt stresses compared to wild-type plants.

In Chapter 3, polyethylene glycol (PEG) was used to mimic drought. Univariate and ME GWA-mapping were performed with phenotypic traits such as project rosette leaf area (PLA), rosette dry weight (DW) or fresh weight (FW), and water content (WC) for Pieris + PEG and Botrytis + PEG responses. A number of SNPs were identified by both methods, of which eight SNPs were 
identified for the consecutive Pieris and PEG responses. The SNPs were associated to genes with IDs At2g36550/At2g36560, and AT3G45910; four common SNPs were found for the response to Botrytis and PEG, the SNPS associated with genes with IDs At1g48670, At1g56280, At2g13690 and a larger region comprising At5g48120 (DI19) - At5g48180. Allelic effects of genes At2g36560 and DI19 in response to combinatorial stress were found. Several SNPs were found to be in linkage disequilibrium with known stress-responsive genes. New roles were found for some of these known stress-responsive genes, e.g. for PRX34 and bZIP25 in their responses to the combined Pieris and PEG treatment, and RMG1 to Botrytis and PEG. We observed antagonistic interaction between the two biotic stress and drought, and a negative effect of the two biotic pre-treatments on subsequent PEG response.

Chapter 4 focused on the consecutive drought and Botrytis infection responses. Univariate GWA-mapping was performed on the residuals of DW and identified the effect of a number of significant SNPs that were associated with the DW plasticity under stresses. The biological functions of six candidate genes were validated under single drought or Botrytis stress and the combinatorial stress using T-DNA insertion mutants and the transcriptome data of the most extreme eight accessions. An allelic effect was observed on MYB46 gene expression under combinatorial stress compared to the single Botrytis treatment. The role of MYB46, PAL4, and HSFA1b in response to the interaction of drought and Botrytis infection was highlighted. Antagonistic interaction between drought and Botrytis infection were observed, as well as a negative effect of drought pretreatment on subsequent response to Botrytis infection.

Chapter 5 reports on whole-transcriptome profile analysis of Arabidopsis Col0 exposed to three single stress triggers (Pieris, Botrytis, drought) and six consecutive combinations. A set of 394 genes were differentially expressed during all three stress conditions, though often in opposite directions. A significant effect of the first-stress-signatures could be identified in the sequential stress profiles, and the effect was often related to responses to phytohormones, strengthening the notion that hormones are general modulators of interactions between different types of stress. 
Chapter 6 focuses on the analysis of the transcriptome data on consecutive biotic (Pieris and Botrytis) and drought stress. Herbivory by Pieris has a much higher impact on the transcriptome during sequential drought and rehydration, than the infection with Botrytis. A set of 42 common genes were found to be responsive to both Pieris + drought and Botrytis + drought stresses and opposite effects of the two biotic pre-treatments were observed on ethylenerelated genes ERF5, ERF6, ERF104 and drought responsive gene P5CS1. Several genes were only differentially expressed under combinatorial stress, showing the unique responses to the consecutive biotic and drought stress. Genes with alternative splicing (AS) variants were identified in different stress conditions, but none of them were found among the differentially expressed genes, showing a separate regulation of gene expression and AS in response to stresses. Phenotypic differences found for the mdar3 and pin7 mutants when compared to wild type suggest that the MDAR3 and PIN7 genes play an important role in the response to the combinatorial stress.

Chapter 7 describes the investigations of the responses of Arabidopsis accessions to the consecutive exposure to Pieris and drought stress. WC is a suitable parameter for evaluation of these interactions. To observe the effect of Pieris pre-treatment on drought responsive genes in response to drought stress, the expression levels of nine drought-responsive genes were measured under different treatments but this showed no correlation with the observed phenotypic variation. In the end, a mode of gene regulatory network is proposed that is sufficient to explain the effect of Pieris pre-treatment on the nine drought responsive genes in response to drought stress.

The study presented here provides an important step towards an in-depth understanding of plant responses to the investigated stress factors and highlights the importance of an array of genes, crucial to the underlying defense processes, as targets for breeding by allele mining, ultimately aimed at improvement of crop tolerance to frequent combinations of stress factors. 


\section{中文摘要}

在自然环境下，生物胁迫和非生物胁迫经常同时发生或先后发生，而且它们常常会对植 物生长带来不利影响。非生物胁迫可以显著影响植物对生物胁迫的响应，其显著程度受到由 于胁迫时间、种类，和严重程度的影响；反之亦然。本论文旨在研究生物胁迫 (菜青虫，或 灰霉病 ) 与非生物胁迫 (干旱 ) 先后处理时对拟南芥生长的影响，所用的主要技术方法为用 全基因组关联分析 (GWAS) 来寻找相关抗性基因位点，以及用 RNA 测序 (RNA sequencing ) 方法来寻找相关抗性基因。研究主要目标是是挖掘双重胁迫下的植物抗性基因。

第二章中，我们用了用多性状数量性状位点 ( QTL ) 分析模型 (MTMMs) 分析了 11 个 单胁迫环境和六个双胁迫环境，找出了响应最显著的 30 个单核苷酸多态性 ( SNPs)。我们 发现在双重胁迫下的 QTL 效应值大于单一胁迫情况下的值。结果表明，在双重胁迫的情况下， 植物启动了广谱抵御机制，导致很多表型性状发生了改变。关联分析鉴定出一些 QTL 位点， 他们在生物/非生物胁迫，或地上/地下胁迫条件下时有相似的或者截然相反的反应。对于生物 胁迫和非生物胁迫，双重胁迫抗性基因例如 RMG1 和 WRKY38 则展现出了既然不同的相应。

在第三章中，聚乙二醇（PEG）-8000 则被用作模拟干旱胁迫环境。表型性状例如莲座 叶面积 ( PLA ) ，莲座干重 ( DW ) ，鲜重 ( FW ) 和水含量 ( WC ) 被用于与单变量和多环 境 GWAs 两种方法分析用。八个相应菜青虫和 PEG 的双重胁迫基因和四个相应灰霉病和 PEG 的双重胁迫基因所描述的两种 GWAs 分析方法所鉴定出来。我们观察到了基因 At2g36560 and DI19 的等位基因效应对于双重胁迫的影响。一些备选基因的生理作用已经 得到 T-DNA 突变体证明。prx34 突变体证明了该基因对菜青虫啃食或 PEG 处理下的植物响 应起正向作用，但是对双重胁迫起负面效应；di19 突变体证明了改基因对灰霉病菌和 PEG 胁 迫单独处理条件下的植物响应有正面作用，但是对双重胁迫没有效应； arf4 突变体证明了该 基因对于灰霉菌和 PEG 单独处理条件下的植物没有效应，但是对双重胁迫有正面效应。 
第四章侧重于连续的干旱和灰霉菌胁迫反应。我们使用了莲座干重 ( DW ) 残差和单变量 GWAs 分析方法，找到了一系列在干旱和灰霉菌先后处理情况下情况下影响表型的重要 SNPs. 我们观察到了 MYB46 基因对于干旱和灰霉菌的的等位基因效应。通过测试备选基因 的 T-DNA 突变体，MYB46，PAL4 和 HSFA1b 对应连续的干旱和灰霉病感染有相应。我们 发现干旱和灰霉菌的交感作用是相反的，并且干旱预处理对随后的灰霉菌感染有负面影响。

第五章报告了拟南芥对于连续的生物胁迫和非生物胁迫反应的的 RNA 测序的结果。拟 南芥分别被菜青虫，灰霉菌，和干旱三种单个胁迫和六个双胁迫所处理过。一共有 394 个基 因对这三种单个胁迫都有反应。预处理胁迫对于随后的胁迫有着明显的影响，而且这种影响 是和植物荷尔蒙有关联的。

第六章侧重于分析针对于菜青虫/灰霉菌和随后的干旱胁迫的 RNA 测序结果。菜青虫对 于随后干旱胁迫的影响力大于灰霉菌对于随之的干旱胁迫。一共有 42 个基因响应于菜青虫和 干旱双胁迫以及灰霉菌和干旱双胁迫。乙烯相关的基因 ERF5，ERF6，ERF104 和干旱胁迫响 应基因 P5CS1 展现出了相反的菜青虫和灰霉菌对于随之的干旱胁迫效应。随后我们鉴定出了 在胁迫环境下展现出了选择性剪切（AS ) 的基因，并且发现这些基因并在胁迫环境下并没有 产生基因表达量的变化。着说明了选择性剪切和基因表达量的变化是对于胁迫环境的两种独 立的相应。突变体例如 $m d a r 3$ 和 pin 7 证明了这两个基因对于菜青虫和干旱双胁迫环境响应 起到了重要作用。

第七章我们调查了拟南芥对于连续的菜青虫和干旱胁迫的反应。表型数据水含量 ( WC ) 是一个非常适合用来评价这两种胁迫的相互作用的参数。我们分别测试了九个干旱抗性基因 在菜青虫和干旱的单独胁迫情况下和双胁迫情况下的的基因表达量，并没有发现在胁迫环境 下的基因表达量的差异和拟南芥的表型有着显著联系。最后，基于这九个干旱基因在胁迫环 境下的不同表达，我们提出了一个应答菜青虫和干旱双胁迫的基因调控网络的模型。 
本文研究提供了对于理解植物对所描述的胁迫环境的响应迈出了重要一步，突出了双重 胁迫的后选抗性基因并描述了潜在的生物防御过程，最终目的在于改善作物对于生物和非生 物胁迫双重胁迫的耐受性。 


\section{Acknowledgements}

The accomplishment of this research involved the support of many people, in the academic formation and social life, in big and small ways. For this, I would like to express my sincere gratitude.

First of all, I would like to thank my promoter Prof. Dr. Maarten Koornneef, co-promotor Dr. Mark Aarts, and Prof. Dr. Bas Zwaan who interviewed me and gave me the opportunity to work on this project. Dear Maarten, thank you very much for your quick response in reviewing all my thesis chapters with detailed comments. I appreciate your patience in answering my questions and I'm grateful for your encouragement to pursue my Ph.D. study. Dear Mark, I admire your intelligence and I respect your belief in fundamental science. I liked the freedom you gave me within the research and I appreciate your advice of attending important scientific events. I hope you can find a balance between work and stress load and I look forward to see more high quality articles from you in future. Dear Bas, I appreciate your guidance and your advice on my project very much when Mark wasn't available. You were always there when I needed help. Especially I want to thank you for getting Pieris rapae larvae for me from outside the Netherlands.

My Ph.D. project is part of the big project "Mining natural genetic variation of combinatorial stress responses of Arabidopsis to identify new tolerance pathways for biotic and abiotic stress" (STW grant, project number: STW10988) and belongs to the program "Learning from Nature" (website http://www.stw.nl/nl/programmas/learning-nature-protect-crops-Ifn). Different research groups from different universities were involved in the program, including ambitious Ph.D. students, post-docs, and professionals. Dr. Nelson Davila and Silvia Coolen, you are not only my collaborators within the combinatorial stress responses project but also are real friends in life. I wish all the best to you and let's keep in touch. Together with other "co-team" members Dr. Manus Thoen, Dr. Karen Kloth, Dr. Willem Kruijer, and Dr. Joost van Heerwaarden, we accomplished our MTMM manuscript. Furthermore I would 
like to thank the professionals involved in this project very much, especially Prof. Dr. Marcel Dicke, Prof. Dr. Joop van Loon, Prof. Dr. Corné Pieterse, Prof. Dr. Fred van Eeuwijk, Dr. Joost Keurentjes, Dr. Saskia van Wees. I also want to express much gratitude to the companies involved in this project, especially to Dr. Marcel Prins, Dr. Alexander Wittenberg, and Dr. Raymond Hulzink (Keygene N.V., Wageningen) for doing RNA sequencing, Dr. Paul Passarinho (Genetwister Technologies B.V., Wageningen), and Dr. Sjoukje Heimovaara (Royal Van Zanten B.V., Rijsenhout). I want to thank Dr. Jan van Kan for the help with preparing Botrytis cinerea spores to use for my experiments and the support he gave me in working with Botrytis. Thank you Dr. Edouard Severing, Dr. Henk Schat and Dr. Sabine Schnabel for the help with analyzing data. Taede Stoker, Gerrit Stunnenberg, and Pauline Sanderson thank you for the help with plant propagation; Leon Westerd and Hans van Pelt thank you for providing Pieris rapae larvae and the initial Botrytis cinerea spores for experimental use.

I would like to express my gratitude to the people from the laboratory of Genetics, for the useful discussion of my project and for the support at my work. I would also like to thank Dr. Mohamed El-Soda, Nihal Öztolan Erol, Dr. Aina Prinzenberg, Dr. Tania Lobato Paulo Serra, Dr. Robert Akkers, Dr. Roxanne van Rooijen, Dr. Yanli Wang, Dr. Ya-Fen Lin, Dr. Pádraic Flood, Dr. Mina Villafort, Charles Moreira, Corrie Hanhart, Frank Becker, Dr. Johanna Bac-Molenaar, Dr. Rik Kooke, Dr. Christina May, Cris Wijnen, Ramon Botet Vaca, and Wytske Nijenhuis and people from other labs; Dr. Christos Kissoudis, Dr. Zhen Wei, and Amin Ebrahimi.

I would not be able to finish this work without the assistance of two talented students Michael Mintgen and Katarzyna Wolinska. Dear Michael, I wish you good luck with your Ph.D. and I have confidence in you solving all difficulties at work. Dear Kasia, you are a hard worker and a bright girl. I admire your scientific attitude and I appreciate your responsibility to the project. Good luck with your study and I am looking forward to read your first scientific paper.

I would like to express great gratitude and respect to Dr. Kaige Zhao. Dear Kaige, you are a hard worker and an enthusiastic learner. You are a respected 
scientist as well as a beloved friend. I admire your indomitable spirit and your open-mindedness. I learned a lot from you during your stay. I wish you all the best with your work and your family. I want to thank Dr. Christian Bachem and Dr. Bjorn Kloosterman, I wouldn't have thought about applying for a Ph.D. position without your inspiration and your encouragement.

Furthermore I want to thank my beloved friends Yanchun Zheng, Sakura Mao, and Lianlan $\mathbf{X u}$. Thank you for the trust, understanding, and support in the past 16 years. Thank you for traveling from China to the Netherlands to prepare my wedding when I was totally frustrated at work. I love you always. To my dear friends who I met in the Netherlands, thank you for the joy and thank you for being there when I needed you, especially to Xianwen Ji, Cheng Liu, Tom van Stein, Hanna van Stein, Yenthe van Stein, Xiaoqian Shi, Tim Kunne, Izan Ramlee, Ameem Yahaya, Aryan Yahaya, Freddy Yeo, Marian Oortwijn, Bart van Tuijl, Weicong Qi, Xi Cheng, and Jihua Cheng.

To my husband Michel Arts, it is my destiny to meet you and to marry you. You played the role of bringing more balance in my life. I can't name any specific reasons to answer the question why I love you so much, I just know that I can't picture my life without you. Veel dank aan mijn schoonouders Ben Arts and Wil Arts-de Graaf, dank jullie voor jullie zorg en ik ben blij met jullie als schoonouders.

Finally, I would like to express my gratitude to my parents He Huang and Lijun Zhao. Especially to my father, I wouldn't have made it so far without his support. 我为有您这样的父亲而骄傲。 


\section{About the author}

Pingping Huang was born on $25^{\text {th }}$ April, 1984 in Chuzhou, P.R.China. She studied a major of Biology during high school at Shenzhen University Normal College Middle School and continued with a bachelor program of Biotechnology at the China Agriculture University (CAU) in P.R.China. During her study at CAU, she worked as a project assistant at the university student council. Subsequently, she continued her study at the Hogeschool van Arnhem en Nijmegen and the Hogeschool Van Hall Larenstein, and obtained the Bachelor degree in International Horticulture and Marketing. In the year 2008, she continued her master study in Plant Science with a specialization in Plant Breeding and Genetic Resources at the Wageningen University (WUR) and there she obtained her MSc degree. During her MSc study, she worked on her master thesis "Identifying and functional characterization of point mutations in sucrose synthase 2 in tomato" with Dr. Christian Bachem and Dr. Antoine Gady at the Laboratory of Plant Breeding (WUR). Additionally she worked on her master minor thesis of "fine mapping of Beta-carotene QTLs in tomato fruit" with Dr. Jim Giovannoni at the Boyce Thompson Institute in the U.S.A. After she completed her master study, shortly she was a guest worker as a technician at the Laboratory of Plant Breeding (WUR). Meanwhile, she applied for and accepted the position of Ph.D. in a 4-year project on "Mining natural genetic variation of combinatorial stress responses of Arabidopsis to identify new tolerance pathways for biotic and abiotic stress" that was funded by the Dutch Technology Foundation (STW) and subsequently became a Ph.D. student at the Laboratory of Genetics. This thesis presents the outcome of her Ph.D. research work. 


\section{List of publications}

\section{Published or Accepted}

1. Manus P.M. Thoen*, Nelson H. Davila Olivas*, Karen J. Kloth*, Silvia Coolen*, PingPing Huang*, Mark G.M. Aarts, Johanna A. Bac-Molenaar, Jaap Bakker, Harro J. Bouwmeester, Colette Broekgaarden, Johan Bucher, Jacqueline Busscher-Lange, Xi Cheng, Emilie F. Fradin, Maarten A. Jongsma, Magdalena M. Julkowska, Joost J.B. Keurentjes, Wilco Ligterink, Corné M.J. Pieterse, Carolien Ruyter-Spira, Geert Smant, Christa Testerink, Björn Usadel, Joop. J.A. van Loon, Johan A. van Pelt, Casper C. van Schaik, Saskia C.M. van Wees, Richard G.F. Visser, Roeland Voorrips, Ben Vosman, Dick Vreugdenhil, Sonja Warmerdam, Gerrie L. Wiegers, Joost van Heerwaarden, Willem Kruijer, Fred A. van Eeuwijk \& Marcel Dicke. (2016) Genetic architecture of plant stress resistance: multi-trait genome-wide association mapping. New Phytologist (Accepted)

2. Silvia Coolen*, Silvia Proietti*, Richard Hickman*, Nelson H. Davila Olivas*, PingPing Huang*, Marcel C. Van Verk, Johan A. Van Pelt, Alexander H.J. Wittenberg, Martin De Vos, Marcel Prins, Joop J.A. Van Loon, Mark G.M. Aarts, Marcel Dicke, Corné M.J. Pieterse and Saskia C.M. Van Wees. (2016) Transcriptome dynamics of Arabidopsis during sequential biotic and abiotic stresses. the Plant Journal. 86(3):249-67. doi:10.1111/tpj.13167

3. Nelson H. Davila Olivas, Silvia Coolen, Pingping Huang, Edouard Severing, Marcel C.van Verk, Richard Hickman, Alexander H. J. Wittenberg, Martin de Vos, Marcel Prinse, Joop J.A. van Loon, Mark G. M. Aarts, Saskia C.M. van Wees, Corné M.J. Pieterseb, Marcel Dicke. (2016) Effect of prior drought and pathogen stress on Arabidopsis transcriptome changes to caterpillar herbivory. New Phytologist. 210: 1344-1356. doi:10.1111/nph.13847

4. Gady A, Vriezen W, Van de Wal M, Huang P, Bovy A, Visser R, Bachem C (2011) Induced point mutations in the phytoene synthase 1 gene cause differences in carotenoid content during tomato fruit ripening. Molecular Breeding: 1-12.

(*shared first authorship)

\section{To be submitted}

1. Pingping Huang, Mohamed El-Soda, Katarzyna W. Wolinska, Nelson H. Davila Olivas, Joop J.A. Van Loon, Marcel Dicke, Kaige Zhao, Jan van Kan, Mark G. M. Aarts. Genome wide association analysis reveals candidate genes controlling a negative effect of biotic stresses on consecutive osmotic stress response.

2. Pingping Huang, Mohamed El-Soda, Katarzyna W. Wolinska, Silvia Coolen, Hans van Pelt, Corné Pieterse, Jan van Kan, Mark G. M. Aarts. Genome-wide association mapping of the Arabidopsis thaliana growth response to the sequential combination of drought and Botrytis cinerea infection. 
3. Pingping Huang, Edouard Severing, Nelson H. Davila Olivas, Silvia Coolen, Marcel C. van Verk, Richard Hickman, Alexander H. J. Wit-tenberg, Martin de Vos, Marcel Prins, Saskia C.M. van Wees, Corné M.J. Pieterse, Joop J.A. van Loon, Marcel Dicke and Mark G. M. Aarts. Opposite interaction effects on the ethylene signalling pathway of two biotic stresses (Botrytis cinerea and Pieris rapae) on subsequent drought stress responses in Arabidopsis thaliana.

4. Pingping Huang, Michael Mintgen, Mark G.M. Aarts. Phenotypic and genetic responses of Arabidopsis thaliana natural accessions to consecutive Pieris rapae herbivory and drought stress. 


\title{
Education certificate
}

\section{Education Statement of the Graduate School Experimental Plant Sciences}

\author{
Issued to: Pingping Huang
}

Date:

7 October 2016

Group: Laboratory of Genetics

University: Wageningen University \& Research centre

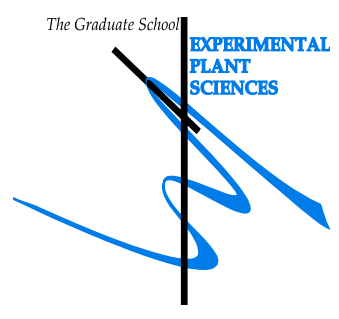

1) Start-up phase

date

- First presentation of your project

Title: Mining Natural Genetic Variation of Combinatorial Stress Responses of Arabidopsis to Identify New Tolerance Pathways for Biotic and Drought Stress

Oct 11,2011

- Writing or rewriting a project proposal

Title: Mining Natural Genetic Variation of Combinatorial Stress Responses of Arabidopsis to Identify New Tolerance Pathways for Biotic and Drought Stress

Jun 2011

- Writing a review or book chapter

- MSc courses

Genetic Analysis, Tools and Concepts (GATC) -GEN 30306

Nov 2011

- Laboratory use of isotopes

\section{2) Scientific Exposure}

- EPS PhD Student Days

EPS PhD student day, Wageningen University \& Research

EPS PhD student day, University of Amsterdam

EPS PhD student Days " Get2Together", Soest, NL

- EPS Theme Symposia

Annual EPS Theme 2 Symposium 'Interaction between Plants and Biotic Agents', Wageningen University \& Research

Annual EPS Theme 3 Symposium 'Metabolism and Adaptation', University of Amsterdam

- Lunteren days and other National Platforms

Annual Meeting "Experimental Plant Sciences", Lunteren, NL Annual Meeting "Experimental Plant Sciences", Lunteren, NL Annual Meeting "Experimental Plant Sciences", Lunteren, NL Annual Meeting "Experimental Plant Sciences", Lunteren, NL

- Seminars (series), workshops and symposia

\section{date}

May 20, 2011

Nov 30, 2012

Jan 29-30, 2015

Feb 10, 2012

Mar 24, 2013

Apr 04-05, 2011

Apr 02-03, 2012

Apr 22-23, 2013

Apr 14-15, 2014 
Plant science seminars

6th Plant-Insect interactions workshop, University of

Nov 23, 2011

Amsterdam

Dec 11,2014

Symposium 'Omics Advances for Academia \& Industry'

- Seminar plus

- International symposia and congresses

Gordon conference 'Salt and Water Stress', USA

Jun 24-29, 2012

Gordon conference 'Salt and Water Stress', USA

European Joint Retreat for PhD students in Plant Sciences, Gif sur Yvette, France

European Joint Retreat for PhD students in Plant Sciences, Norwich, UK

Aug 03-08, 2014

Jul 05-08, 2011

Aug 15-17, 2012

\section{- Presentations}

Poster: Annual Meeting "Experimental Plant Sciences", Lunteren

Poster: PhD Retreat, France

Talk: Discovery combinatorial stress tolerant genes - STW meeting

Poster: Annual Meeting "Experimental Plant Sciences", Lunteren

Poster: Gordon conference 'Salt and Water Stress'

Poster: PhD retreat UK

Poster: Discovering combinatorial stress tolerant genes - STW meeting

Talk: Discovery combinatorial stress tolerant genes - STW meeting

Talk: Discovery combinatorial stress tolerant genes - STW meeting

Poster Annual Meeting "Experimental Plant Sciences", Lunteren

Talk: Discovery combinatorial stress tolerant genes - STW meeting

Talk: Discovery combinatorial stress tolerant genes - STW meeting

Talk: Discovery combinatorial stress tolerant genes - STW meeting

Poster: Annual Meeting "Experimental Plant Sciences", Lunteren

Poster: Gordon conference salt and water stress

Talk: Discovery combinatorial stress tolerant genes - STW meeting

Talk: Discovery combinatorial stress tolerant genes - STW meeting

Apr 04-05, 2011

Jul 05-08, 2011

Sep 29, 2011

Apr 02-03, 2012

Jun 24-29, 2012

Aug 15-17, 2012

Sep 18, 2012

Sep 29, 2012

Jan 29, 2013

Apr 22-23, 2013

Jun 18,2013

Sep 19, 2013

Mar 28, 2014

Apr 14-15, 2014

Aug 03-08, 2014

Sep 26, 2014

Mar 10, 2015

- IAB interview

Meeting with a member of the International Advisory Board of EPS

Sep 29, 2014 


\begin{tabular}{|lc|}
\hline 3) In-Depth Studies & $\underline{\text { date }}$ \\
EPS courses or other PhD courses & \\
PhD summer school 'Environmental Signalling' & Aug 22-24, 2011 \\
Introduction to R for Statistical Analysis & Oct 24-25, 2011 \\
PhD Summerchool on Environmental Signaling & Aug 22-24, 2011 \\
Mixed model based QTL mapping in GenStat & May 14-16, 2012 \\
PhD summer school 'Natural Variation of Plants' & Aug 21- 24, 2012 \\
Bioinformatics 'A User's Approach' & Aug 26-30, 2013 \\
Current Trends in Phylogenetics & Oct 14-18, 2013 \\
Transcription Factors and Transcriptional Regulations & Dec 17-19, 2013 \\
Journal club & \\
Literature discussion at Genetics & $2011-2015$ \\
\hline Individual research training & \\
\hline
\end{tabular}

Subtotal In-Depth Studies

9.7 credits*

4) Personal development

date

- Skill training courses

Competence Assessment

May 24, 2011

Project and Time Management

Oct-Dec 2012

English for Professional Purposes

Jan-Apr 2012

- Organisation of PhD students day, course or conference

- Membership of Board, Committee or PhD council

Subtotal Personal Development

3.1 credits*

TOTAL NUMBER OF CREDIT POINTS*

52.7

Herewith the Graduate School declares that the PhD candidate has complied with the educational requirements set by the Educational Committee of EPS which comprises of a minimum total of 30 ECTS credits

* A credit represents a normative study load of 28 hours of study. 


\section{Learning from Nature to protect crops}

Plants are under the constant threat of biotic and abiotic stresses. Yet, devastating pests and diseases only rarely occur in nature and plants have managed to sustain for millions of years in this hostile environment. This is due to and has resulted in a tremendous degree of natural variation in mechanisms that plants exploit to defend themselves against pathogens and insects and to deal with abiotic stresses. In agriculture, however, we have exploited only very little of this diversity of defenses and as a consequence environment-malignant pesticides remain a dominant method to control pests and diseases. The current threat of climatic changes and limiting resources for agriculture (water, fertilizer) require improved resistance to abiotic stresses.

\section{Ambition and goal}

With this multidisciplinary and innovative STW programme we want to mine the natural reservoir of plant defense mechanisms. This will be done by using state-of-the-art high-throughput technologies to explore the natural potential and exploit mechanisms, genes and markers to develop novel resistance mechanisms against biotic and abiotic stresses for plant breeding.

In nature plants have co-evolved with a large variety of attackers. Therefore, wild species, such as Arabidopsis thaliana, harbour a fantastic reservoir of natural adaptive mechanisms to respond to (a)biotic stresses that to date have not been systematically explored. In the past decade, Arabidopsis has been adopted world-wide as the ideal model for plant science and an impressive molecular genetic toolbox has since been developed (e.g. the full genome sequence, the availability of well-characterized Arabidopsis populations, fullgenome microarrays and metabolomics protocols). Hence, exploring natural variation in the defense responses of Arabidopsis to a large variety of (a)biotic stresses will yield important new insights into how plants selectively adapt to stresses, and provide novel concepts for sustainable agriculture and resistance breeding. 


\section{Objectives}

1. To explore natural varation in resistance to abiotic and biotic stresses in Arabidopsis populations through an integrated multidisciplinary approach.

2. To identify mechanisms underlying natural resistance to abiotic and biotic stresses in Arabidopsis

3. To develop methods to analyze complex datasets on different types of resistance

4. To exploit information gained on natural variation in Arabidopsis to identify molecular markers that can assist in breeding for resistance to abiotic and biotic stresses in crop plants.

\section{Focus and results at the end of the programme}

To this end Arabidopsis ecotype and RIL populations can be exploited to analyze the degree of resistance to a diversity of microbial pathogens, herbivorous insects and abiotic stresses and their interaction. Using large-scale bioinformatics this information can be integrated with transcriptomics and metabolomics, to select genotypes and lines that can be used for in-depth analysis of the resistance mechanisms. The information gained from this comprehensive approach will lead to the identification of genes and molecular markers for different resistance mechanisms. These mechanisms will be characterized at the molecular, biochemical and physiological level and can subsequently be used to screen large numbers of lines of various crop species for orthologous genes involved in similar resistance mechanisms.

\section{Innovation}

Never before has the natural variation in plant defenses against different biotic and abiotic stresses and their interaction been investigated in such a comprehensive, multidisciplinary programme. To date, solutions to individual (a)biotic stresses have been sought. However, this has not resulted in a systems approach that results in durable solutions for a range of stresses. 
The research described in this thesis was conducted at the laboratory of Genetics at Wageningen University and Plant Microbe Interactions group at Utrecht University. This research was supported by The Netherlands Organization for Scientific Research (NWO) through the Technology Foundation, Perspective Programme 'Learning from Nature' [STW grant number 10988].

(c) 2016 Pingping Huang

Cover design: Lianlan Xu, nortaking@qq.com

Thesis layout: Pingping Huang

Printed by Digiforce || Proefschriftmaken.nl || 